\title{
Natural Gas
}

\section{Imports and Exports}

\section{First Quarter Report}

1995

Prepared By:

U.S. Department of Energy

Office of Fuels Programs

Office of Fossil Energy

Washington, DC 20585

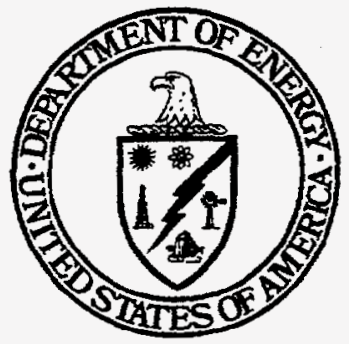

\section{DISCLAIMER}

This report was prepared as an account of work sponsored by an agency of the United States Government. Neither the United States Government nor any agency thereof, nor any of their employees, makes any warranty, express or implied, or assumes any legal liability or responsibility for the accuracy, completeness, or usefulness of any information, apparatus, product, or process disclosed, or represents that its use would not infringe privately owned rights. Reference herein to any specific commercial product, process, or service by trade name, trademark, manufacturer, or otherwise does not necessarily constitute or imply its endorsement, recommendation, or favoring by the United States Government or any agency thereof. The views and opinions of authors expressed herein do not necessarily state or reflect those of the United States Government or any agency thereof. 


\section{DISCLAIMER}

Portions of this document may be illegible in electronic image products. Images are produced from the best available original document. 


\section{SUMMARY}

The Office of Fuels Programs prepares quarterly reports summarizing the data provided by companies authorized to import or export natural gas. Companies are required, as a condition of their authorizations, to file quarterly reports with the OFP. This report is for the first quarter of 1995 (January - March).

Attachment $A$ shows the percentage of takes to maximum firm contract levels and the weighted average per unit price for each of the long-term importers during the five most recent reporting quarters.

Attachment B shows volumes and prices of gas purchased by long-term importers and exporters during the past 12 months.

Attachment $C$ shows volume and price information pertaining to gas imported on a short-term or spot market basis.

Attachment $\mathrm{D}$ shows the gas exported on a shortterm or spot market basis to Canada and Mexico.

First Quarter Highlights: Natural Gas exports to Mexico continued their robust pace set during the second half of 1994, totaling $17.8 \mathrm{Bcf}$. This compares with 4.4 Bcf exported during the first quarter of 1994 and represents a year-to-year increase of 305 percent. Canadian imports climbed 15 percent above last year's level, despite pipeline capacity constraints and a return to more normal winter weather. Distrigas and Pan National Gas Sales, Inc., resumed importing Algerian LNG for the first time since September 1994. The two LNG importers received a total of 3 tankers during the first quarter of 1995, importing an aggregate volume of $7.7 \mathrm{Bcf}$. This compares with 7 tankers and 22.7 Bcf during the first quarter of 1994.

During the first three months of 1995, data indicates gas imports increased by about 12 percent over the 1994 level (664 vs. 741 Bcf), with Canadian imports increasing by 15 percent and Algerian imports decreasing by 66 percent. During the same time period, exports increased by 3 percent ( 40.6 vs. $41.7 \mathrm{Bct}$ ). Exports to Canada decreased by 67 percent (21.6 vs. 7.2 Bcf). Exports to Japan increased by 14 percent (14.6 Bcf vs. $16.7 \mathrm{Bcf}$ ). Exports to Mexico increased by 305 percent (4.4 vs. $17.8 \mathrm{Bct}$ ).
Long-term imports for the quarter totaled 356.6 Bcf, including $351.5 \mathrm{Bcf}$ from Canada. This represents a one percent increase over the first quarter of 1994. The average price of these supplies was $\$ 1.75 / \mathrm{MMBtu}$, which was 8 cents or 4 percent less than the preceding quarter. Under other long-term import arrangements, Distrigas Corporation imported 5.1 Bcf of Algerian LNG at an average landed price of \$2.21/MMBtu. Pan National Gas Sales, Inc. did not import any Algerian LNG under its long-term contracts.

During the first quarter, 104 companies used shortterm authorizations to import $383.2 \mathrm{Bcf}$ of natural gas. This volume represents a 24 percent increase over the first quarter of 1994. Mexican imports totaled $0.3 \mathrm{Bcf}$ during the quarter.

The average price of Canadian natural gas under short-term import arrangements was $\$ 1.18 / \mathrm{MMBtu}$, compared with $\$ 1.35 / \mathrm{MMBtu}$ during the preceding quarter. Approximately 47 percent of the shortterm sales were made at Eastport, Idaho, at an average price of $\$ 0.93 / \mathrm{MMBtu} ; 15$ percent at Port of Morgan, Montana, at $\$ 1.07 / \mathrm{MMBtu}$; 13 percent at Sumas, Washington, at $\$ 1.34 / \mathrm{MMBtu} ; 11$ percent at Noyes, Minnesota, at $\$ 1.47 / \mathrm{MMBtu} ; 5$ percent at Niagara Falls, New York, at $\$ 1.85 / \mathrm{MMBtu} ; 3$ percent at Waddington, New York, at $\$ 1.70 / \mathrm{MMBtu}$; and 6 percent at various other entry points, at \$1.71/MMBtu.

In addition, a total of 22 short-term export authorizations were used, exporting a total of 25 Bcf of gas. Ten authorizations were used to export about 7.2 Bcf of gas to Canada, at an average price of $\$ 1.87 / \mathrm{MMBtu}$. Under 12 other authorizations, about $17.8 \mathrm{Bcf}$ of gas was exported to Mexico at an average price of $\$ 1.39 / \mathrm{MMBtu}$.

This quarter's feature report focuses on Canadian natural gas imports into New England. OFP invites ideas from the public on future topics dealing with North American natural gas import/export trade. Such suggestions should be left on OFP's electronic bulletin board.

The quarterly report and any future revisions will be resident on OFP's Electronic Bulletin Board at (202) 586-7853. All general queries concerning this report should be made to Yvonne Caudillo at (202) $586-4587$. 
NATURAL GAS IMPORTS

Volumes and Weighted Average Prices

By Point of Entry and Type of Authorization

$(01 / 01 / 95$ - 03/31/95)

VOLUME

(MMCF)
WEIGHTED

AVERAGE

PRICE

(\$MMBTU)

\section{POINT OF ENTRY}

\section{LONG-TERM AUTHORIZATIONS}

Babb, Montana

Champlain, New York

Eastport, Idaho

Everett, Massachusetts

Grand Island, New York

Highgate Springs, Vermont

International Falls, Minnesota

Lake Charles, Louisiana

Massena, New York

Niagara Falls, New York

North Troy, Vermont

Noyes, Minnesota

Port of Del Bonita, Montana

Port of Morgan, Montana

Portal, North Dakota

Sumas, Washington

Waddington, New York

Warroad, Minnesota

Whitlash, Montana

$\begin{array}{rl}450.27 & 1.33 \\ 4,123.26 & 3.09 \\ 54,920.30 & 1.04 \\ 5,131.67 & 2.21^{*} \\ 5,594.68 & 2.53 \\ 2,553.46 & 2.13 \\ 1,736.95 & 2.04 \\ 0.00 & 0.00^{* * *} \\ 4,776.23 & 2.77 \\ 48,497.38 & 2.35 \\ 2,746.10 & 2.39 \\ 64,222.86 & 1.84 \\ 24.87 & 1.36 \\ 78,116.75 & 1.16 \\ 6.18 & 2.47 \\ 21,475.95 & 1.78 \\ 60,136.79 & 2.27 \\ 222.34 & 2.36 \\ 1,917.94 & 1.39 \\ & \\ \mathbf{3 5 6 , 6 5 3 . 9 8} & \mathbf{1 . 7 5}\end{array}$

TOTAL LONG-TERM IMPORTS

$356,653.98$

\section{SHORT-TERM AUTHORIZATIONS}

Babb, Montana

Champlain, New York

Detroit, Michigan

Eastport Idaho

Grand Island, New York

Hidalgo, Texas

Highgate Springs, Vermont

Lake Charles, Louisiana

Massena, New York

Niagara Falls, New York

North Troy, Vermont

Noyes, Minnesota

Penitas, Texas

Port of Morgan, Montana

Portal, North Dakota

St. Clair, Michigan

Sumas, Washington

Waddington, New York

Whitlash, Montana

* Average landed price

** Average tailgate price

Note: Import figures in this table may vary slightly from sum of components in report due to independent rounding in calculations. 


\section{QUARTERLY FOCUS:}

\section{MARKET PENETRATION OF GAS IMPORTS INTO NEW ENGLAND}

- Historically, natural gas use in New England has played a relatively minor role in meeting the region's energy supply needs due to the region's geographical location at the end of the interstate natural gas transmission system. The region's energy supply needs have been largely met by petroleum. As recently as 1987, petroleum use equaled about 63 percent of the total energy consumed in New England while gas use accounted for only 13 percent of its energy use. In comparison, nationwide use of petroleum and natural gas in 1987 equaled 43 percent and 23 percent of total energy consumption, respectively [EIA, State Energy Data Report 1992 (May 1994)].

- $\quad$ Although New England's past use of natural gas has been relatively minor, natural gas import supplies have been present in the region for 30 years. Since 1966, Vermont Gas Systems, Inc., a local distribution company (LDC) located in northwest Vermont, has been importing Canadian gas from TransCanada PipeLines Ltd., or its marketing affiliate, Western Gas Marketing, Ltd. Due to the LDC's isolated location, there are no interstate pipelines available to transport domestic gas sources to its market area; as a consequence, Vermont Gas is totally reliant on Canadian natural gas for its system's supplies.

- In addition to Canadian natural gas imports into New England, liquefied natural gas (LNG) imports from Algeria have been used since 1968 as a small but important source of gas for the region, particularly as a winter peak-shaving fuel. Boston Gas Company imported the first LNG supplies into the country in November 1968, and Distrigas Corporation, with its LNG import terminal located at Everett, Massachusetts, has been importing Algerian LNG since November 1971. LNG imports were introduced into New England primarily to meet winter demand requirements which exceeded the amount of domestic gas that could be delivered to the region by pipeline or from storage.

- From the late 1960 's to late 1984, most of the gas imports into New England were made by either Vermont Gas, or Distrigas. There were some small volumes of Canadian gas imported at the Niagara Falls, New York, entry point that found their way to New England, but the volumes were relatively insignificant.

- Following the oil import supply disruptions of the late 1970 's, the New England states took steps in the early 1980's to promote the diversification their energy sources, both in terms of supply mix and source. One of the steps was to advocate increased use of Canadian natural gas. 
Figure 1 shown on the next page depicts the chronology in the growth of natural gas imports into New England over the last three decades. It lists nine events which facilitated the growth in the use of natural gas imports in the region. From 1966 to the fall of 1984, the use of natural gas imports in New England grew from less than 1 Bcf to over $42 \mathrm{Bcf}$. As stated earlier, Distrigas and Vermont Gas were the suppliers of virtually all of the imported gas marketed in New England during this time period, with Distrigas importing the majority of the volumes. From 1966 to 1984, imports by Vermont Gas grew from less than $1 \mathrm{Bcf}$ to about $5 \mathrm{Bcf}$, and Algerian LNG imports, beginning in 1968, grew to over 36 Bcf by 1984. Figure 1 illustrates that the growth of gas imports during this period was uneven; this was due to the often significant annual fluctuations in the imports of Algerian LNG by Distrigas.

- The 4th event listed in Figure 1, the commencement of imports by Boundary, Inc., was a watershed in the utilization of Canadian natural gas in the U.S. Northeast, including New England. Boundary Gas, Inc. was formed in 1980 by 14 natural gas distribution companies in New England, New York, and New Jersey, for the sole purpose of securing long-term firm Canadian gas supplies to supplement traditional sources of supply in the region. The Boundary Gas Project marked the first time a group of local distribution companies (LDC), including nine from New England, directly negotiated long-term pipeline gas supplies to supplement their customary pipeline deliveries. The Project also provided for new pipeline capacity, thereby improving the logistical ability of these LDCs to secure additional foreign and domestic supplies.

- The drop in natural gas imports shown in Figure 1 during the mid-1980's was due to the suspension of Algerian LNG imports by Distrigas in 1985 due to a price dispute with Sonatrach, the Algerian national oil and gas company. Distrigas did not resume importing from Algeria until 1988.

- The 6th event shown in Figure 1, the construction and operation of the Iroquois Gas Transmission System, greatly increased the presence of Canadian natural gas in the U.S. Northeast. Today, approximately half of this system's capacity is dedicated to supplying New England. Under 34 long-term gas supply contracts, almost 123 Bcf of Canadian gas per year flows through this system into New England.

- As indicated in Figure 1, New England now receives Canadian natural gas at four international entry points. During 1994, natural gas importers delivered supplies to New England under 43 long-term contracts (longer than 2 years) at these four international entry points. The 43 long-term contracts have an aggregate daily contract demand of 598.9 MMcf, or 218.6 Bcf per year. 
- $\quad$ Figure 2 shows the substantial growth in natural gas use in New England over the past 8 years, as well as the region's increasing reliance on imported gas supplies to meet the incremental growth in demand.

- From 1987 to 1994 , total natural gas consumption grew from 374.4 to $532.0 \mathrm{Bcf}$, or over 42 percent. During the same time period, natural gas imports marketed in New England grew from 21.2 to $238.1 \mathrm{Bcf}$, or a ten-fold increase. Virtually the entire incremental growth in demand during this period was supplied by imported natural gas supplies.

- $\quad$ Figure 2 shows that natural gas imports as a percentage of total natural gas consumption in the region grew from a modest 5.7 percent in 1987 to well over 40 percent during the past two years. Today, New England relies more on natural gas imports to meet its supply needs than any other census region in the country.

Figure 2

\section{Sources of Natural Gas Marketed in New England}

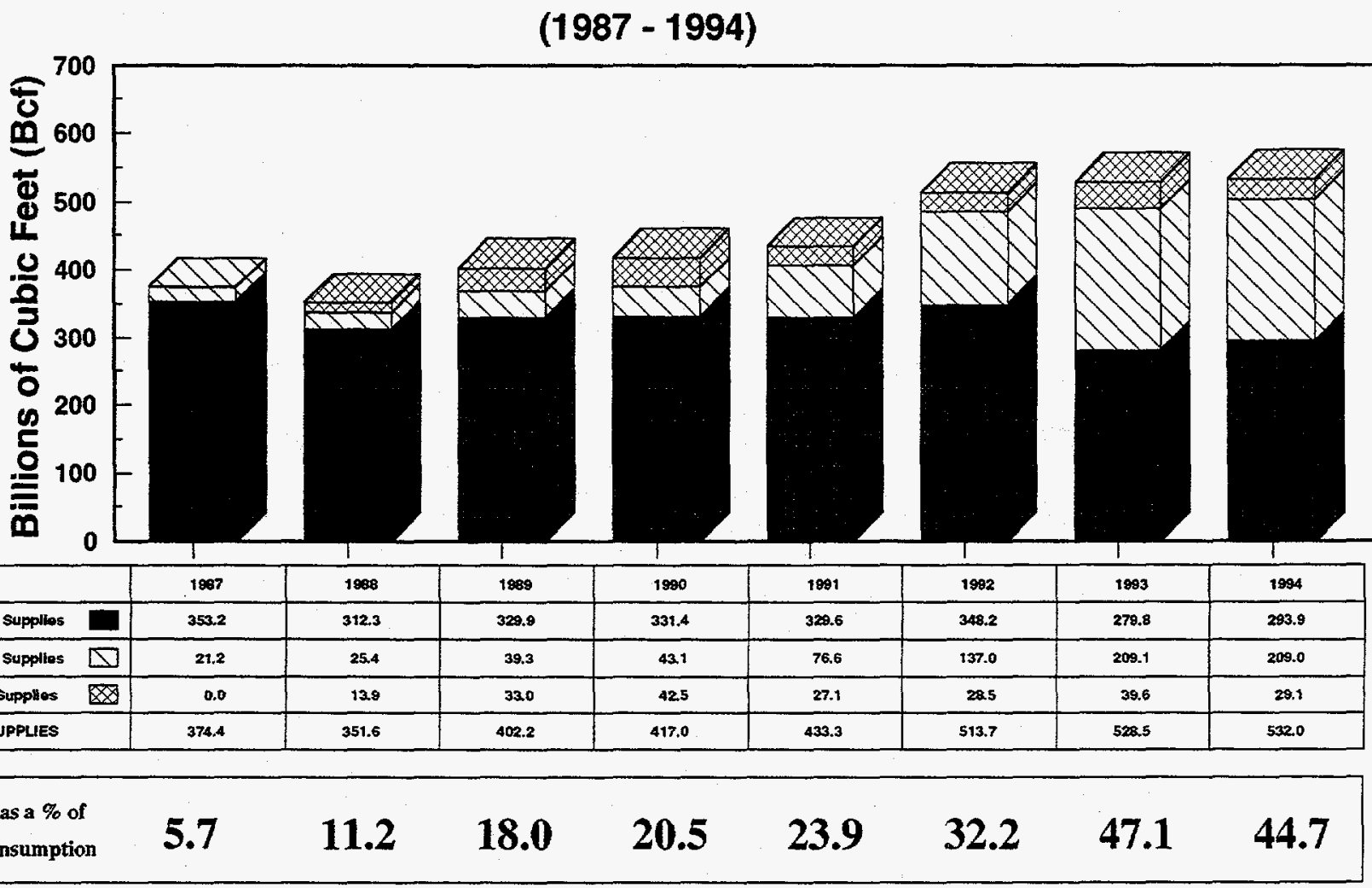

Sources: Natural gas consumption data from 1987 - 1993 came from EIA's Natural Gas Annual (DOE/EIA - 0131); 1994 consumption figure is an estimate from EIA; Import data are derived from company filings made with OFP. 
- Figures 3 through 5 present snapshots of natural gas import activity in New England during 1994.

- $\quad$ Figure 3 shows the distribution of natural gas imports marketed during 1994 in the 6 states comprising New England.

- As shown, the bulk of the imports destined for sale in New England is sold in the state of Massachusetts. About 45 percent of the Canadian gas import volumes and 84 percent of the Algerian LNG volumes marketed in New England are sold in this state.

Figure 3

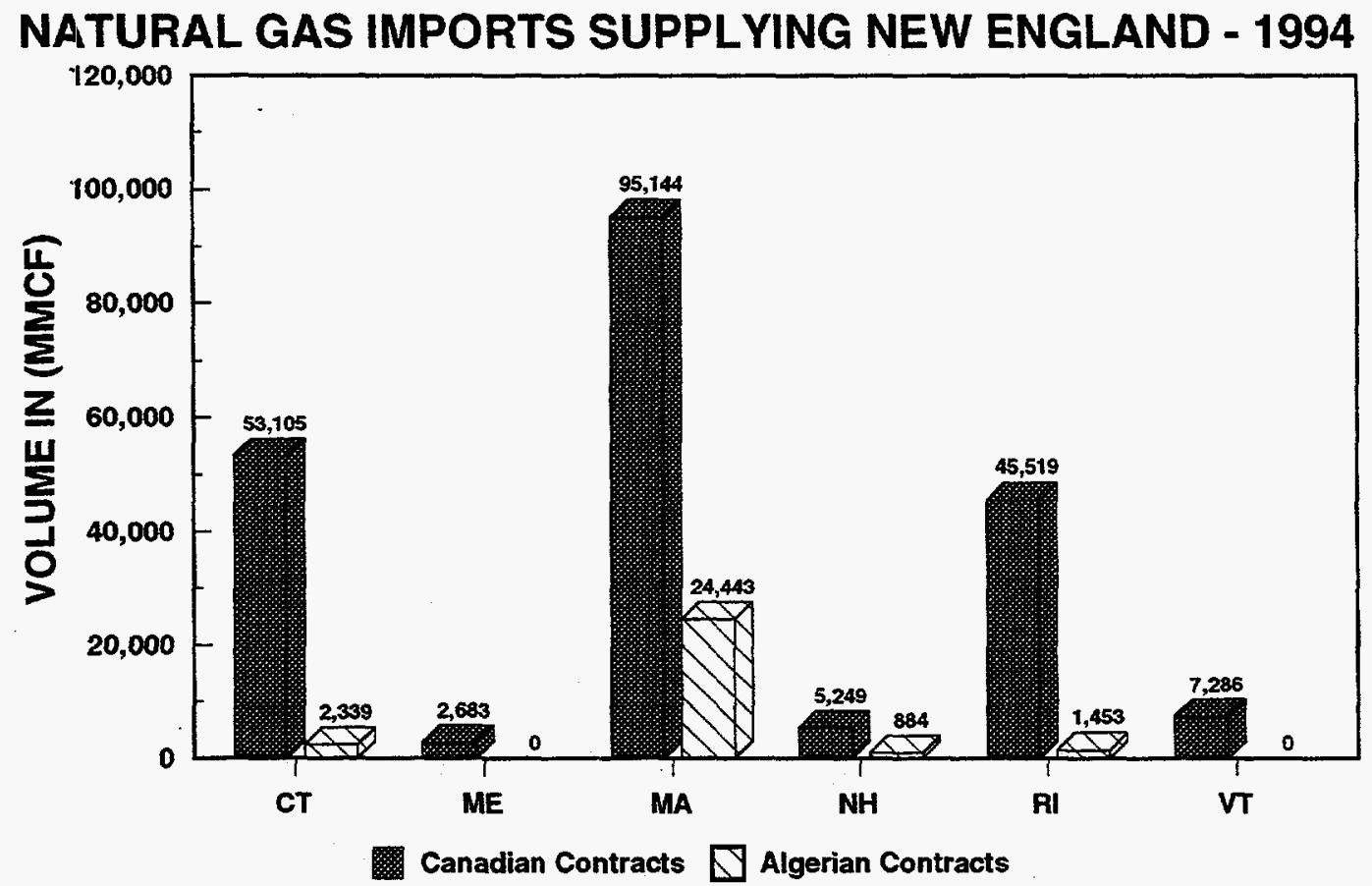

Source: Quarterly filings by natural gas importers to OFP.

- Figure 4 shows the total volume of Canadian natural gas imported into New England under long-term contracts in 1994, as well as the percentages of this volume that were imported by class of importer; e.g., local distribution company.

- During 1994 the weighted average international border price for Canadian natural gas imported into New England under long-term contracts was \$2.44 per MMBtu. This compares with a $\$ 2.00$ per MMBtu average price for all Canadian long-term gas supplies imported into the United States during 1994.

- The 190.8 Bcf of natural gas sold in New England under long-term contracts represents 91.3 percent of the total Canadian gas volumes marketed in New England during 1994. The remaining gas imports ( 8.7 percent) were sold under contracts of two years or less. The high percentage of imports coming into New England under long-term contracts is in sharp contrast with the country as a whole. In 1994, 53 percent of all Canadian gas 
imports into the United States were purchased under long-term contracts, while the other 47 percent were made under short-term arrangements. The long-term nature of the contracts serving New England can be directly attributed to the underlying need for firm long-term gas supply arrangements to support the pipelines which were built to serve this region over the past 10 years.

-

Figure 5 estimates New England's reliance on imported natural gas on a state-by-state basis. The percentage shown for each state represents the portion of the state's gas supplies derived from natural gas imports. Although overall regional reliance on natural gas imports during 1994 is estimated to be about 45 percent, there is considerable differences among the 6 states which comprise New England. For example, the state of Vermont does not consume great volumes of natural gas, but virtually all of its supplies come from Canada. In spite of the fact that Massachusetts consumes over half of natural gas imports in the region, in 1994 only 39 percent of its supplies came from imports.

\section{Figure 4}

\section{CANADIAN NATURAL GAS IMPORTS INTO NEW ENGLAND UNDER LONG-TERM CONTRACTS}

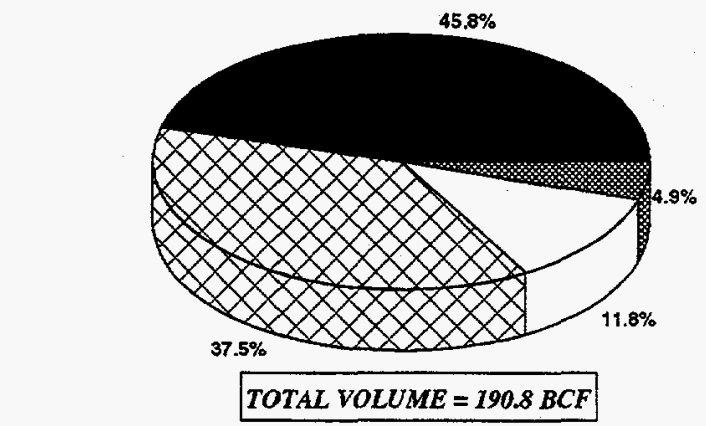

Local Dist. Cos. $\square$ Non-Utility Gen. Fac $\square$ Interstate Pipeline Note: Boundary Gas, Inc. Imports are shown as LDCs. Source: Quarterly fillngs by natural gas Importers to OFP
ESTIMATED STATE RELIANCE ON NATURAL GAS IMPORTS DURING 1994

(As a Percentage of Total State Gas Consumption)

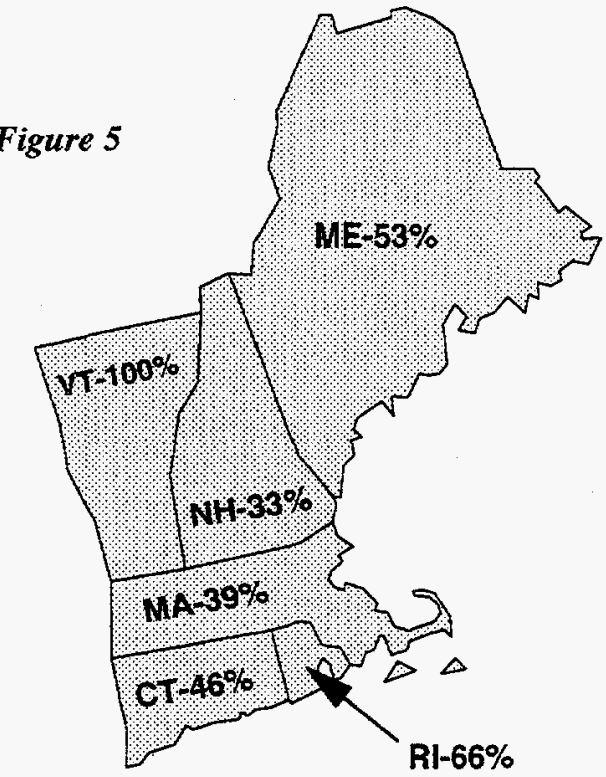

Sources: Total state gas consumptlon data are estimates from EIA, Offlce of Oll and Gas, Reserves and Naturat Gas Division, Data Operations Branch; natural gas Import consumption data are from quarterly flings submitted to OFP from gas importers. 
Page - 1

\section{LONG-TERM IMPORTS}

ACTUAL IMPORTS AS A PERCENT OF AUTHORIZED VOLUMES

\begin{tabular}{rrrrr}
1994 & 1994 & 1994 & 1994 & 1995 \\
qtr & qtr & \multicolumn{1}{c}{ qtr } & qtr & \multicolumn{1}{c}{ qtr } \\
$\# 1$ & $\# 12$ & $\# 3$ & $\# 4$ & $\# 1$ \\
& & & & \\
69 & 79 & 79 & 89 & 91 \\
100 & N/A & N/A & N/A & N/A \\
100 & 100 & 100 & 100 & 100 \\
102 & 100 & 100 & 86 & 100 \\
100 & 100 & 99 & 98 & 100 \\
99 & 99 & 99 & 99 & 99 \\
100 & 100 & 100 & 100 & 100 \\
100 & 100 & 100 & 100 & 100 \\
100 & 100 & 98 & 100 & 99 \\
100 & 100 & 100 & 100 & 100 \\
98 & 89 & 95 & 96 & 96 \\
34 & 30 & 14 & 64 & 60 \\
98 & 98 & 98 & 98 & 98 \\
100 & 100 & 100 & 98 & 100 \\
96 & 96 & 96 & 96 & 96 \\
99 & 99 & 99 & 99 & 99 \\
101 & 102 & 101 & 102 & 102 \\
100 & 100 & 100 & 100 & 100 \\
100 & 100 & 100 & 100 & 100 \\
98 & 98 & 91 & 89 & 97 \\
97 & 84 & 87 & 86 & 93 \\
101 & 101 & 96 & 98 & 98 \\
100 & 100 & 100 & 96 & 100 \\
100 & 100 & 100 & 100 & 100 \\
100 & 101 & 101 & 101 & 101 \\
101 & 101 & 101 & 101 & 101 \\
100 & 100 & 100 & 100 & 100 \\
100 & 100 & 100 & 100 & 100 \\
99 & 99 & 99 & 99 & 99 \\
101 & 101 & 101 & 101 & 101 \\
100 & 100 & 100 & 100 & 100 \\
100 & 100 & 99 & 100 & 100 \\
99 & 100 & 100 & 100 & 100 \\
100 & 100 & 100 & 100 & 100 \\
99 & 104 & 104 & 104 & 104 \\
& & & &
\end{tabular}

\section{5}

11

91

/A

100

100

100

99

100

100

99

100

96

98
100

96

99

102

100

00

97

98

100

01

100

01

100

100

104
WEIGHTED AVERAGE PRICE (\$MMBTU) OF IMPORTS

$\begin{array}{ccccc}\begin{array}{c}1994 \\ \text { qtr }\end{array} & \begin{array}{c}1994 \\ \text { qtr }\end{array} & \begin{array}{c}1994 \\ \text { qtr }\end{array} & \begin{array}{c}1994 \\ \text { qtr }\end{array} & \begin{array}{c}1995 \\ \text { qtr }\end{array} \\ \text { \#1 } & \# 2 & \# 3 & \# 4 & \text { \#1 } \\ 2.27 & 2.27 & 2.27 & 2.28 & 2.28 \\ 2.35 & \text { N/A } & \text { N/A } & \text { N/A } & \text { N/A } \\ 2.71 & 2.18 & 2.21 & 2.25 & 2.32 \\ 3.00 & 2.53 & 2.40 & 2.55 & 2.38 \\ 2.67 & 2.19 & 2.18 & 2.23 & 2.30 \\ 2.69 & 2.18 & 2.21 & 2.25 & 2.32 \\ 2.69 & 2.18 & 2.21 & 2.25 & 2.32 \\ 2.86 & 2.18 & 2.24 & 2.25 & 2.32 \\ 2.71 & 2.18 & 2.21 & 2.25 & 2.32 \\ 2.73 & 2.18 & 2.18 & 2.25 & 2.32 \\ 2.64 & 2.18 & 2.08 & 2.11 & 2.20 \\ 2.54 & 2.25 & 3.18 & 1.78 & 1.58 \\ 2.27 & 1.81 & 1.72 & 1.76 & 1.51 \\ 2.22 & 2.18 & 2.18 & 1.77 & 1.76 \\ 2.69 & 2.18 & 2.21 & 2.25 & 2.32 \\ 2.69 & 2.18 & 2.15 & 2.25 & 2.32 \\ 2.86 & 2.18 & 2.23 & 2.25 & 2.32 \\ 2.71 & 2.18 & 2.21 & 2.25 & 2.32 \\ 2.73 & 2.18 & 2.17 & 2.25 & 2.32 \\ 1.73 & 1.72 & 1.85 & 1.76 & 1.25 \\ 1.73 & 1.72 & 1.84 & 1.85 & 1.25 \\ 1.73 & 1.76 & 1.77 & 1.81 & 1.23 \\ 2.71 & 2.18 & 2.21 & 2.24 & 2.32 \\ 2.71 & 2.18 & 2.21 & 2.25 & 2.32 \\ 2.69 & 2.18 & 2.21 & 2.25 & 2.32 \\ 2.69 & 2.18 & 2.15 & 2.25 & 2.32 \\ 2.86 & 2.18 & 2.23 & 2.25 & 2.32 \\ 2.71 & 2.18 & 2.21 & 2.25 & 2.32 \\ 2.73 & 2.18 & 2.17 & 2.25 & 2.32 \\ 2.69 & 2.18 & 2.21 & 2.25 & 2.32 \\ 2.69 & 2.18 & 2.15 & 2.25 & 2.32 \\ 2.86 & 2.18 & 2.23 & 2.25 & 2.32 \\ 2.72 & 2.18 & 2.21 & 2.25 & 2.32 \\ 2.73 & 2.18 & 2.17 & 2.25 & 2.32 \\ 2.58 & 2.45 & 2.10 & 1.76 & 1.83\end{array}$

AG-ENERGY, L.P./Home Oil Company Limited

ANR PIPELINE COMPANYIProGas Limited

BOSTON GAS COMPANY/Alberta Northeast Gas (WGM Ltd. 1)

BOSTON GAS COMPANYIEsso Resources Canada Limited

BOUNDARY GAS, INC. $/$ TransCanada Pipelines Limited

BROOKLYN UNION GAS COMPANY/Alberta NE Gas (AEC Oil \& Gas Company)

BROOKLYN UNION GAS COMPANY/Alberta Northeast Gas (Atcor)

BROOKL.YN UNION GAS COMPANY/Alberta Northeast Gas (ProGas)

BROOKLYN UNION GAS COMPANY/Alberta Northeast Gas (WGM LId. 1)

BROOKLYN UNION GAS COMPANY/Alberta Northeast Gas (WGM Ltd. 2)

CASCADE NATURAL GAS CORPORATION/Mobil Oil Canada, Ltd.

CASCADE NATURAL GAS CORPORATION/Westcoast Gas Services

CASCADE NATURAL GAS CORPORATION/Westcoast Gas Services

CASCADE NATURAL GAS CORPORATION/Westcoast Gas Services

CENTRAL HUDSON COMPANYIAlberta NE Gas (AEC Oil \& Gas Company)

CENTRAL HUDSON COMPANY/Alberta Northeast Gas (Atcor)

CENTRAL HUDSON COMPANYIAlberta Northeast Gas (ProGas)

CENTRAL HUDSON COMPANY/Alberta Northeast Gas (WGM Ltd. 1)

CENTRAL HUDSON COMPANY/Alberta Northeast Gas (WGM Ltd. 2)

CITY OF BURBANK, CALIFORNIA/Westcoast Gas Services

CITY OF GLENDALE, CALIFORNIA/Westcoast Gas Services

CITY OF PASADENA, CALIFORNIAWWestcoast Gas Services

COLONIAL NATURAL GAS/Alberta Northeast Gas (WGM Ltd. 1)

COMMONWEALTH GAS COMPANYIAlberta Northeast Gas (WGM Ltd. 1)

CONNECTICUT NATURAL GAS/Alberta NE Gas (AEC Oil \& Gas Company)

CONNECTICUT NATURAL GAS/Alberta Northeast Gas (Atcor)

CONNECTICUT NATURAL GAS/Alberta Northeast Gas (ProGas)

CONNECTICUT NATURAL GAS/Alberta Northeast Gas (WGM Lid. 1)

CONNECTICUT NATURAL GAS/Alberta Northeast Gas (WGM Ltd. 2)

CONSOLIDATED EDISON COMPANY OF N.Y./Alberta NE Gas (AEC Oil \& Gas)

CONSOLIDATED EDISON COMPANY OF N.Y./Alberta NE Gas (Atcor)

CONSOLIDATED EDISON COMPANY OF N.Y./Alberta NE Gas (ProGas)

CONSOLIDATED EDISON COMPANY OF N.Y./Alberta NE Gas (WGM Ltd. 1)

CONSOLIDATED EDISON COMPANY OF N.Y./Alberta NE Gas (WGM Ltd. 2)

CONSOLIDATED EDISON COMPANY OF N.Y./Amoco Canada 


\section{LONG-TERM IMPORTS}

ACTUAL IMPORTS AS A PERCENT OF AUTHORIZED VOLUMES

\begin{tabular}{|c|c|c|c|c|}
\hline $\begin{array}{c}1994 \\
\text { qtr } \\
\# 1\end{array}$ & $\begin{array}{c}1994 \\
\text { qtr } \\
\# 2\end{array}$ & $\begin{array}{c}1994 \\
\text { qtr } \\
\# 3\end{array}$ & $\begin{array}{c}1994 \\
\text { qtr } \\
\# 4\end{array}$ & $\begin{array}{c}1995 \\
\text { qtr } \\
\sharp i \overline{1}\end{array}$ \\
\hline 95 & 100 & 100 & N/A & N/A \\
\hline 95 & 100 & 100 & N/A & N/A \\
\hline 95 & 100 & N/A & N/A & N/A \\
\hline 95 & 100 & 75 & N/A & $N / A$ \\
\hline 95 & 100 & $N / A$ & N/A & $\mathrm{N} / \mathrm{A}$ \\
\hline 94 & 100 & N/A & N/A & N/A \\
\hline 99 & 101 & 99 & 99 & 100 \\
\hline 57 & 53 & 66 & 23 & N/A \\
\hline 57 & 54 & 67 & 18 & $N / A$ \\
\hline 59 & 58 & 67 & 66 & 88 \\
\hline N/A & $\mathrm{N} / \mathrm{A}$ & $N / A$ & 92 & 89 \\
\hline 115 & 111 & 138 & 77 & 91 \\
\hline N/A & N/A & $N / A$ & N/A & N/A \\
\hline N/A & N/A & N/A & N/A & $\mathrm{N} / \mathrm{A}$ \\
\hline 100 & 100 & 100 & 100 & 101 \\
\hline 86 & 87 & 77 & 90 & 93 \\
\hline 86 & 87 & 88 & 86 & 66 \\
\hline 99 & 99 & 99 & 99 & 100 \\
\hline 99 & 99 & 99 & 89 & 99 \\
\hline 100 & 100 & 94 & 92 & 99 \\
\hline 101 & 101 & 100 & 99 & 101 \\
\hline 94 & 99 & 94 & 87 & 98 \\
\hline 99 & 99 & 83 & 91 & 98 \\
\hline 100 & 100 & 100 & 80 & 100 \\
\hline 100 & 99 & 95 & 99 & N/A \\
\hline 77 & 74 & 78 & 74 & 78 \\
\hline 75 & 73 & 65 & 73 & 73 \\
\hline 110 & 109 & 109 & 110 & N/A \\
\hline 84 & 85 & 78 & 68 & 65 \\
\hline 80 & 90 & 95 & 90 & $N / A$ \\
\hline 89 & 81 & 87 & 60 & 64 \\
\hline 72 & 80 & 82 & 32 & 4 \\
\hline 100 & 100 & 100 & 100 & 100 \\
\hline 97 & 97 & 96 & 93 & 101 \\
\hline 96 & 76 & 74 & 77 & 93 \\
\hline
\end{tabular}

WEIGHTED AVERAGE PRICE (\$MMBTU) OF IMPORTS

\begin{tabular}{|c|c|c|c|c|}
\hline $\begin{array}{c}1994 \\
\text { qtr } \\
\pm 11\end{array}$ & $\begin{array}{c}1994 \\
\mathrm{qtr} \\
\# 2\end{array}$ & $\begin{array}{c}1994 \\
\text { qtr } \\
\# \underline{\# 3}\end{array}$ & $\begin{array}{c}1994 \\
\text { qtr } \\
\# 4\end{array}$ & $\begin{array}{c}1995 \\
\text { qtr } \\
\# 1\end{array}$ \\
\hline 1.74 & 1.70 & 2.09 & N/A & N/A \\
\hline 1.82 & 1.88 & 2.10 & N/A & N/A \\
\hline 1.77 & 1.72 & N/A & $\mathrm{N} / \mathrm{A}$ & N/A \\
\hline 1.75 & 1.98 & 2.12 & $N / A$ & N/A \\
\hline 1.69 & 1.66 & N/A & N/A & N/A \\
\hline 1.71 & 1.68 & N/A & $N / A$ & $N / A$ \\
\hline 2.25 & 1.96 & 1.82 & 1.73 & 1.65 \\
\hline 2.80 & 2.74 & 2.71 & 5.36 & N/A \\
\hline 2.80 & 2.71 & 2.71 & 5.36 & N/A \\
\hline 2.80 & 2.73 & 2.71 . & 2.63 & 2.19 \\
\hline$N / A$ & N/A & $N / A$ & 2.30 & 2.19 \\
\hline 2.80 & 2.73 & 2.71 & 2.84 & 2.20 \\
\hline 2.49 & 2.18 & 2.16 & $N / A$ & 2.21 \\
\hline 2.71 & 2.14 & $N / A$ & N/A & N/A \\
\hline 2.98 & 2.64 & 2.77 & 2.57 & 2.68 \\
\hline 2.76 & 2.94 & 3.10 & 2.86 & 2.94 \\
\hline 1.84 & 1.84 & 1.93 & 1.93 & 1.93 \\
\hline 2.71 & 2.18 & 2.21 & 2.25 & 2.32 \\
\hline 2.71 & 2.18 & 2.21 & 2.25 & 2.32 \\
\hline 2.67 & 2.59 & 2.56 & 2.63 & 2.41 \\
\hline 2.44 & 2.30 & 2.23 & 2.39 & 2.38 \\
\hline 2.62 & 2.39 & 2.47 & 2.56 & 2.40 \\
\hline 2.55 & 2.35 & 2.47 & 2.26 & 2.19 \\
\hline 2.11 & 1.68 & 1.44 & 1.60 & 1.34 \\
\hline 2.23 & 1.73 & 1.54 & 1.29 & $N / A$ \\
\hline 2.54 & 2.54 & 2.56 & 2.54 & 2.61 \\
\hline 2.30 & 2.29 & 2.35 & 2.24 & 2.31 \\
\hline 2.15 & 2.13 & 2.12 & 2.00 & N/A \\
\hline 2.32 & 2.53 & 2.42 & 2.39 & 2.23 \\
\hline 2.57 & 2.45 & 2.42 & 2.40 & N/A \\
\hline 1.78 & 2.00 & 1.87 & 1.77 & 1.55 \\
\hline 2.65 & 2.49 & 2.53 & 2.44 & 2.47 \\
\hline 2.59 & 2.38 & 2.29 & 2.16 & 1.88 \\
\hline 2.75 & 2.46 & 2.43 & 2.33 & 2.25 \\
\hline 3.13 & 3.35 & 3.36 & 3.46 & 3.35 \\
\hline
\end{tabular}

CONSUMERS POWER COMPANYMGM LIT.

CONSUMERS POWER COMPANY1/Norcen Energy Resources LId. CONSUMERS POWER COMPANY2/Husky Oil

CONSUMERS POWER COMPANY3/Shell Canada Limited

CONSUMERS POWER COMPANY4/Poco Petroleums Litd.

CONSUMERS POWER COMPANY5/North Canadian Oils, Lid.

CRESTAR ENERGY MARKETING CORPORATION/Crestar Energy

DARTMOUTH POWER ASSOCIATES, L.P./Anderson Oil \& Gas Inc.

DARTMOUTH POWER ASSOCIATES, L.P./Canadian Natural Gas Resources

DARTMOUTH POWER ASSOCIATES, L.P./Excel Energy Inc.

DARTMOUTH POWER ASSOCIATES, L.P./Home Oil Company Limited

DARTMOUTH POWER ASSOCIATES, L.P./Remington Energy LId.

DISTRIGAS CORPORATION (88-37-LNG)/Sonatrach

DISTRIGAS CORPORATION (89-16-LNG)/Sonatrach

ELIZABETHTOWN GAS COMPANYNGM Ltd.

ENCOGEN FOUR PARTNERS, L.P./Sceptre Resources Limited

ENCOGEN NORTHWEST, L.P./CanWest Gas Supply, Inc.

ENERGYNORTH, INC./Alberta Northeast Gas (WGM Lid. 1)

ESSEX COUNTY GAS CO./Alberta Northeast Gas (WGM Litd. 1)

FULTON COGENERATION ASSOCIATES/Star Oil \& Gas Limited

GRANITE STATE GAS TRANSMISSION, INC./Direcl Energy Marketing Ltd.

GRANITE STATE GAS TRANSMISSION, INC./Shell Canada Limited

GRANITE STATE GAS TRANSMISSION, INC./Shell Canada Limited

GREAT FALLS GAS COMPANY/Shell Canada Limited

IGI RESOURCES, INC.Mabil Oil Canada

INDECK ENERGY SERVICES OF OSWEGO, INC./Indeck Gas Supply Corp. INDECK-YERKES ENERGY SERVICES, INC./Indeck Gas Supply Corp.

INDECK-YERKES LIMITED PARTNERSHIP/Northstar Energy Corporation KAMINE/BESICORP CARTHAGE L.P./Renaissance Energy Ltd.

KAMINE/BESICORP NATURAL DAM L.P./North Canadian Marketing Inc. KAMINE/BESICORP SOUTH GLENS FALLS L.P./Renaissance Energy Ltd. KAMINE/BESICORP SYRACUSE L.P./North Canadian Marketing Inc. KCS ENERGY MARKETING, INC./Ramarro Resources Ltd.

LG\&E WESTMORELAND RENSSELAER/WGM LId.

LOCKPORT ENERGY ASSOCIATES, L.P./ProGas Limited 
Page - 3

\section{LONG-TERM IMPORTS}

\section{ACTUAL IMPORTS AS A PERCENT OF AUTHORIZED VOLUMES}

\section{IMPORTER/EXPORTER}

LONG ISLAND LIGHTING COMPANY/Alberta NE Gas (AEC Oil \& Gas) LONG ISLAND LIGHTING COMPANY/Alberta Northeast Gas (Atcor) LONG ISLAND LIGHTING COMPANY/Alberta Northeast Gas (ProGas) LONG ISLAND LIGHTING COMPANY/Alberta Northeast Gas (WGM Ltd. 1) LONG ISLAND LIGHTING COMPANY/Alberta Northeast Gas (WGM LId. 2) MEGAN-RACINE ASSOCIATES, INC.IWGM LId.

MICHIGAN CONSOLIDATED GAS COMPANY/WGM Ltd.

MIDLAND COGENERATION VENTURE1/Norcen Energy Resources Lid.

MIDLAND COGENERATION VENTURE2/Shell Canada Limited

MIDLAND COGENERATION VENTURE3/Canterra Energy Limited

MIDLAND COGENERATION VENTURE4/TransCanada Pipelines Limited

MIDLAND COGENERATION VENTURE5/Poco Petroleums Ltd.

MIDLAND COGENERATION VENTURE6/North Canadian Oils, L.td.

MINNEGASCO, INC, $/$ TransCanada Pipelines Limited

N.Y. STATE ELECTRIC \& GAS CO./Alberta NE Gas (AEC Oil \& Gas Co.)

N.Y. STATE ELECTRIC \& GAS CO./Alberta Northeast Gas (Atcor)

N.Y. STATE ELECTRIC \& GAS CO./Alberta Northeast Gas (ProGas)

N.Y. STATE ELECTRIC \& GAS CO./Alberta Northeast Gas (WGM Ltd. 2)

N.Y. STATE ELECTRIC \& GAS CO./Crestar Energy

N.Y. STATE ELECTRIC \& GAS CO./Progas Limited

NATIONAL FUEL GAS DISTRIBUTION CORPORAT/Alberta NE Gas (WGM Ltd)

NATURAL GAS PIPELINE COMPANY OF AMERICA/ProGas Limited

NATURAL GAS PIPELINE COMPANY OF AMERICAWGM LId.

NEW ENGLAND POWER COMPANY/Renaissance Energy Limited

NEW ENGLAND POWER COMPANY/Sceptre Resources Limited

NEW ENGLAND POWER COMPANYTTalisman Energy Ine.

NEW ENGLAND POWER COMPANY/Transwest Energy Inc.

NEW JERSEY NATURAL GAS COMPANY/Alberta NE Gas (AEC Oil \& Gas Company) NEW JERSEY NATURAL GAS COMPANY/Alberta Northeast Gas (Atcor)

NEW JERSEY NATURAL GAS COMPANY/Alberta Northeast Gas (ProGas)

NEW JERSEY NATURAL GAS COMPANY/Alberta Northeast Gas (WGM Ltd. 1)

NEW JERSEY NATURAL GAS COMPANY/Alberta Northeast Gas (WGM Lid. 2)

NIAGARA MOHAWK POWER CORPORATION/WGM Ltd.

NORTH JERSEY ENERGY ASSOCIATES/ProGas Limited

NORTHEAST ENERGY ASSOCIATES/ProGas Limited

\begin{tabular}{rrrrr}
1994 & 1994 & 1994 & 1994 & 1995 \\
qtr & qtr & qtr & qtr & \multicolumn{1}{c}{ qtr } \\
$\# 1$ & $\underline{\# 2}$ & $\# 3$ & $\underline{\# 4}$ & $\# 1$ \\
& & & & \\
99 & 99 & 99 & 99 & 99 \\
98 & 99 & 99 & 99 & 99 \\
100 & 100 & 100 & 100 & 100 \\
100 & 100 & 100 & 100 & 100 \\
100 & 100 & 100 & 100 & 104 \\
97 & 67 & 80 & 73 & 89 \\
92 & 90 & 89 & 88 & 91 \\
65 & 65 & 65 & 88 & 100 \\
100 & 100 & 100 & 100 & 100 \\
100 & 100 & 100 & 100 & 100 \\
100 & 100 & 100 & 100 & 100 \\
100 & 100 & 100 & 100 & 72 \\
100 & 42 & 50 & 50 & 50 \\
99 & 78 & 69 & 93 & 99 \\
102 & 102 & 102 & 102 & 102 \\
99 & 99 & 99 & 99 & 99 \\
101 & 101 & 100 & 93 & 101 \\
99 & 99 & 99 & 90 & 99 \\
80 & 49 & 23 & 80 & 93 \\
58 & 90 & 89 & 77 & 76 \\
100 & 100 & 100 & 100 & 100 \\
66 & $\mathrm{~N} / \mathrm{A}$ & $\mathrm{N} / \mathrm{A}$ & $\mathrm{N} / \mathrm{A}$ & $\mathrm{N} / \mathrm{A}$ \\
88 & 98 & 95 & 97 & 100 \\
80 & 68 & 46 & 68 & 74 \\
58 & 52 & 32 & 60 & 74 \\
57 & 52 & 20 & 60 & 74 \\
57 & 37 & 32 & 42 & $\mathrm{~N} / \mathrm{A}$ \\
102 & 102 & 102 & 102 & 102 \\
99 & 99 & 99 & 99 & 99 \\
100 & 100 & 100 & 100 & 100 \\
100 & 100 & 99 & 100 & 100 \\
99 & 99 & 99 & 99 & 99 \\
100 & 100 & 100 & 100 & 100 \\
95 & 91 & 90 & 76 & 90 \\
100 & 100 & 100 & 99 & 100
\end{tabular}

WEIGHTED AVERAGE PRICE (\$MMBTU) OFIMPORTS

\begin{tabular}{|c|c|c|c|c|}
\hline $\begin{array}{c}1994 \\
\text { qtr } \\
\# 1\end{array}$ & $\begin{array}{c}1994 \\
\text { qtr } \\
\# 2\end{array}$ & $\begin{array}{c}1994 \\
\text { qtr } \\
\sharp 3\end{array}$ & $\begin{array}{c}1994 \\
\text { qtr } \\
\# 4\end{array}$ & $\begin{array}{c}1995 \\
\text { qtr } \\
\# 1\end{array}$ \\
\hline 2.69 & 2.18 & 2.21 & 2.25 & 2.32 \\
\hline 2.69 & 2.18 & 2.15 & 2.25 & 2.32 \\
\hline 2.86 & 2.18 & 2.23 & 2.25 & 2.32 \\
\hline 2.71 & 2.18 & 2.21 & 2.25 & 2.32 \\
\hline 2.73 & 2.18 & 2.17 & 2.25 & 2.32 \\
\hline 2.63 & 3.15 & 2.82 & 2.84 & 2.57 \\
\hline 2,38 & 2.08 & 1.85 & 1.72 & 1.61 \\
\hline 1.85 & 1.83 & 1.82 & 1.81 & 1.81 \\
\hline 1.76 & 1.76 & 1.74 & 1.73 & 1.73 \\
\hline 1.90 & 1.90 & 1.90 & 1.89 & 1.91 \\
\hline 1.76 & 1.74 & 1.73 & 1.72 & 1.71 \\
\hline 1.94 & 1.83 & 1.97 & 1.99 & 2.20 \\
\hline 2.39 & 2.91 & 2.79 & 2.82 & 2.85 \\
\hline 2.68 & 2.02 & 1.86 & 1.90 & 1.82 \\
\hline 2.69 & 2.18 & 2.21 & 2.25 & 2.32 \\
\hline 2.69 & 2.18 & 2.15 & 2.25 & 2.32 \\
\hline 2.86 & 2.18 & 2.23 & 2.24 & 2.32 \\
\hline 2.73 & 2.18 & 2.17 & 2.24 & 2.32 \\
\hline 3.18 & 3.13 & 2.90 & 2.68 & 2.13 \\
\hline 2.46 & 2.39 & 2.59 & 2.69 & 3.01 \\
\hline 2.71 & 2.19 & 2.21 & 2.24 & 2.31 \\
\hline 2.22 & $N / A$ & $N / A$ & $N / A$ & N/A \\
\hline 2.51 & 2.12 & 1.95 & 1.81 & 1.70 \\
\hline 2.82 & 2.71 & 2.77 & 2.47 & 2.33 \\
\hline 2.49 & 2.40 & 2.31 & 2.07 & 1.73 \\
\hline 2.56 & 2.49 & 2.44 & 2,34 & 2.44 \\
\hline 2.86 & 2.86 & 2.32 & 2.10 & $N / A$ \\
\hline 2.69 & 2.18 & 2.21 & 2.25 & 2.32 \\
\hline 2.69 & 2.18 & 2.15 & 2.25 & 2.32 \\
\hline 2.86 & 2.18 & 2.23 & 2.25 & 2.32 \\
\hline 2.71 & 2.18 & 2.21 & 2.25 & 2.32 \\
\hline 2.73 & 2.18 & 2.17 & 2.25 & 2.32 \\
\hline 2.31 & 2.05 & 1.92 & 1.88 & 1.80 \\
\hline 2.62 & 2.52 & 2.57 & 2.77 & 2.58 \\
\hline 3.11 & 3.07 & 3.07 & 3.08 & \\
\hline
\end{tabular}




\section{LONG-TERM IMPORTS}

\section{ACTUAL IMPOATS AS A PERCENT OF AUTHORIZED VOLUMES}

\begin{tabular}{|c|c|c|c|c|}
\hline $\begin{array}{c}1994 \\
\text { qtr } \\
\# 1\end{array}$ & $\begin{array}{l}1994 \\
\text { qtr } \\
\# 2\end{array}$ & $\begin{array}{c}1994 \\
\text { qtr } \\
\# 3\end{array}$ & $\begin{array}{c}1994 \\
\text { qtr } \\
\sharp 4\end{array}$ & $\begin{array}{c}1995 \\
\text { qtr } \\
\# 1\end{array}$ \\
\hline 85 & 62 & 17 & 50 & 59 \\
\hline 70 & 52 & 42 & 56 & 65 \\
\hline 127 & 39 & 26 & 82 & 130 \\
\hline 33 & 10 & 5 & 22 & 35 \\
\hline 100 & 87 & 98 & 89 & 101 \\
\hline 98 & 99 & 100 & 87 & 100 \\
\hline 99 & 98 & 100 & 59 & 99 \\
\hline N/A & N/A & N/A & 99 & 99 \\
\hline N/A & N/A & N/A & 99 & 99 \\
\hline 100 & 100 & 89 & 93 & 106 \\
\hline 100 & 100 & 90 & 96 & 65 \\
\hline 90 & 55 & 52 & 95 & 43 \\
\hline 66 & 39 & 42 & 95 & 96 \\
\hline 86 & 28 & 12 & 83 & 103 \\
\hline 51 & 50 & N/A & N/A & N/A \\
\hline 100 & 98 & 96 & 99 & 99 \\
\hline 99 & 99 & 97 & 100 & 103 \\
\hline 88 & 97 & 97 & 100 & 103 \\
\hline 100 & 102 & 102 & 100 & 100 \\
\hline 100 & 99 & 99 & 100 & 100 \\
\hline 100 & 90 & 101 & 100 & 100 \\
\hline N/A & $N / A$ & 83 & 100 & 100 \\
\hline 100 & 98 & 94 & 78 & 83 \\
\hline 98 & 133 & 65 & N/A & N/A \\
\hline 70 & 52 & 81 & 73 & 85 \\
\hline 72 & 54 & 84 & 68 & 85 \\
\hline 75 & 29 & 15 & 38 & 41 \\
\hline 84 & 82 & 60 & N/A & N/A \\
\hline 98 & 92 & 100 & 99 & 97 \\
\hline N/A & N/A & 0 & 2 & 3 \\
\hline 80 & 69 & 73 & 78 & 92 \\
\hline 88 & 88 & 88 & 73 & 51 \\
\hline N/A & N/A & N/A & N/A & N/A \\
\hline 100 & 100 & 100 & 100 & 100 \\
\hline$N / A$ & 98 & N/A & 100 & 99 \\
\hline
\end{tabular}

\section{(ass}

65

5

9

3

99

100

100

33

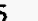

5

2

99
WEIGHTED AVERAGE PRICE (\$MMBTU) OF IMPORTS

\begin{tabular}{|c|c|c|c|c|}
\hline $\begin{array}{c}1994 \\
\text { qtr } \\
\# 1\end{array}$ & $\begin{array}{c}1994 \\
\text { qtr } \\
\# 2\end{array}$ & $\begin{array}{c}1994 \\
\text { qtr } \\
\mathbb{1 1 3}\end{array}$ & $\begin{array}{c}1994 \\
\text { qtr } \\
\# 4\end{array}$ & $\begin{array}{c}1995 \\
\text { qtr } \\
\# 1\end{array}$ \\
\hline 2.36 & 2.55 & 4.43 & 2.72 & 2.62 \\
\hline 2.68 & 2.87 & 3.11 & $2: 49$ & 2.04 \\
\hline 2.25 & 3.02 & 3.59 & 2.44 & 2.25 \\
\hline 2.77 & 4.78 & 7.73 & 3.18 & 2.74 \\
\hline 2.41 & 2.46 & 2.38 & 2.19 & 1.77 \\
\hline 2.75 & 2.06 & 1.96 & 1.99 & 1.75 \\
\hline 2.41 & 2.16 & 2.05 & 2.11 & 1.83 \\
\hline N/A & $N / A$ & N/A & 1.69 & 1.47 \\
\hline N/A & $N / A$ & N/A & 1.79 & 1.58 \\
\hline 1.76 & 1.59 & 1.57 & 1.72 & 1.38 \\
\hline 1.80 & 1.61 & 1.63 & 1.77 & 1.97 \\
\hline 2.09 & 2.25 & 2.18 & 1.62 & 2.49 \\
\hline 2.36 & 2.02 & 1.72 & 1.46 & 1.26 \\
\hline 2.14 & 2.45 & 3.68 & 1.52 & 1.55 \\
\hline 2.27 & 1.84 & $\mathrm{~N} / \mathrm{A}$ & N/A & $N / A$ \\
\hline 2.01 & 1.99 & 1.82 & 1.75 & 0.68 \\
\hline 2.11 & 1.95 & 1.74 & 1.61 & 1.57 \\
\hline 1.58 & 1.33 & 1.18 & 1.09 & 1.00 \\
\hline 1.99 & 1.98 & 1.96 & 1.96 & 1.96 \\
\hline 1.99 & 1.99 & 1.96 & 1.96 & 1.96 \\
\hline 1.98 & 1.98 & 1.96 & 1.96 & 1.93 \\
\hline N/A & $\mathrm{N} / \mathrm{A}$ & 2.59 & 2.38 & 2.32 \\
\hline 2.85 & 2.89 & 2.83 & 2.85 & 2.85 \\
\hline 1.75 & 1.75 & 1.83 & N/A & N/A \\
\hline 2.43 & 2.77 & 2.33 & 2.52 & 2.45 \\
\hline 2.45 & 2.80 & 2.47 & 2.71 & 2.57 \\
\hline 3.08 & 4.91 & 7.70 & 3.05 & 3.01 \\
\hline 1.70 & 1.70 & 1.70 & $N / A$ & N/A \\
\hline 2.24 & 2.03 & 1.97 & 1.97 & 1.99 \\
\hline N/A & $N / A$ & 2.60 & 2.60 & 2.46 \\
\hline 3.14 & 3.37 & 3.23 & 3.19 & 3.35 \\
\hline 2.28 & 2.15 & 2.10 & 2.55 & 3.12 \\
\hline 2.47 & 2.43 & 2.42 & 2.23 & 2.16 \\
\hline 2.71 & 2.18 & 2.21 & 2.25 & 2.32 \\
\hline N/A & 1.21 & $N / A$ & 1.62 & 1.32 \\
\hline
\end{tabular}

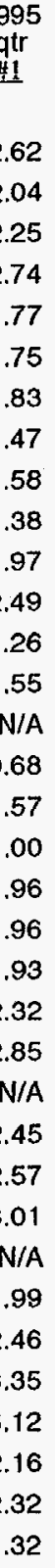

NORTHERN MINNESOTA UTILITIESNGM LId.

NORTHERN MINNESOTA UTILITIES - EASTERNMGGM LId.

NORTHERN MINNESOTA UTILITIES - WESTERN/WGM LId.

NORTHERN MINNESOTA UTILITIES/CITY OF WAWGM LId.

NORTHERN STATES POWER COMPANY/Amoco Canada Petroleum Company Lid.

NORTHERN STATES POWER COMPANY/Canadian Occidental Petroloum Co. Lid.

NORTHERN STATES POWER COMPANY/ProGas Limited

NORTHERN STATES POWER COMPANY (MINNESOT/Amoco Canada Pet. Co. Ltd.

NORTHERN STATES POWER COMPANY (MINNESOTMWGM Ltd.

NORTHWEST NATURAL GAS COMPANY/Amoco Canada Petroleum Company Ltd.

NORTHWEST NATURAL GAS COMPANY/CanWest Gas Supply

NORTHWEST NATURAL GAS COMPANY/Poco Petroleums Limited

NORTHWEST NATURAL GAS COMPANY/Summit Resources Limited

NORTHWEST NATURAL GAS COMPANYMeslcoast Gas Sevices

NORTHWEST NATURAL GAS COMPANYMestcoast Gas Services

NW ALASKAN PIPELINE CO-Pacific intersta/Pan-Alberta Gas Ltd.

NW ALASKAN PIPELINE CO-Pan-Alberta Gas/Pan-Alberta Gas Lid.

NW ALASKAN PIPELINE CO-Pan-Alberta Gas/Pan-Alberta Gas Ltd.

OCEAN STATE POWER/ProGas Limited

OCEAN STATE POWER II/ProGas Limited

OCEAN STATE POWER IIWGM Ltd.

ORANGE AND ROCKLAND UTILITIES, INC.NWascana Energy Marketing

ORCHARD GAS CORPORATION/ProGas Limited

PAN NATIONAL GAS SALES, INC./Sonatrading

PAWTUCKET POWER ASSOCIATES/Home Oil Company Limited

PAWTUCKET POWER ASSOCIATES/Tarragon Oil \& Gas Limited

PEOPLES NATURAL GAS COMPANYWGM LId.

PEPPERELL POWER ASSOCIATES, L.P.MGM LId.

POCO PETROLEUM INC./MI Resources, InC.

PORTAL MUNICIPAL GAS/SASKENERGY INC.

POWER CITY PARTNERS, L.P./Husky Oil Operations Ltd.

PROGAS U.S.A. INC.IProGas Limited

PROJECT ORANGE ASSOCIATES, L.P.INoranda, Inc.

PUBLIC SERVICE ELECTRIC \& GAS/Alberta Northeast Gas (WGM Ltd. 1)

RENAISSANCE ENERGY (U.S.) INC./Renaissance Energy LId. 


\section{LONG-TERM IMPORTS}

ACTUAL IMPORTS AS A PERCENT OF AUTHOBIZED VOLUMES

\begin{tabular}{|c|c|c|c|c|}
\hline $\begin{array}{c}1994 \\
\text { qtr } \\
\# 1\end{array}$ & $\begin{array}{c}1994 \\
\text { qtr } \\
\# 2\end{array}$ & $\begin{array}{c}1994 \\
\text { qtr } \\
\# 3\end{array}$ & $\begin{array}{c}1994 \\
\text { qtr } \\
\# 4\end{array}$ & $\begin{array}{c}1995 \\
\text { qtr } \\
\# 1\end{array}$ \\
\hline 100 & 100 & 100 & 91 & 94 \\
\hline 95 & 104 & 91 & 96 & 99 \\
\hline 96 & 96 & 95 & 99 & 99 \\
\hline 77 & 90 & 85 & 99 & 85 \\
\hline 99 & 101 & 75 & N/A & N/A \\
\hline 93 & 100 & 99 & 99 & 100 \\
\hline 98 & 99 & 95 & 98 & 99 \\
\hline 2 & 32 & 79 & 80 & 85 \\
\hline N/A & N/A & N/A & 67 & 91 \\
\hline N/A & N/A & N/A & 67 & 91 \\
\hline$N / A$ & $N / A$ & N/A & 67 & 84 \\
\hline 90 & 77 & 73 & 55 & 84 \\
\hline N/A & N/A & $N / A$ & 52 & 88 \\
\hline 100 & 89 & 74 & 85 & 90 \\
\hline 67 & 66 & 67 & 68 & 67 \\
\hline 48 & 47 & 48 & 57 & 61 \\
\hline 98 & 96 & 97 & 99 & 98 \\
\hline 67 & 66 & 67 & 92 & 105 \\
\hline 99 & 99 & 99 & 99 & 99 \\
\hline 101 & 101 & 101 & 101 & 101 \\
\hline 100 & 100 & 100 & 100 & 100 \\
\hline 100 & 100 & 100 & 117 & 150 \\
\hline 100 & 100 & 100 & 100 & $N / A$ \\
\hline 54 & 30 & 20 & 40 & 55 \\
\hline 102 & 90 & 95 & 45 & 73 \\
\hline 80 & 76 & 74 & 77 & 80 \\
\hline N/A & 54 & 94 & 77 & 75 \\
\hline N/A & 93 & 85 & 89 & 77 \\
\hline$N / A$ & 96 & 95 & 96 & 94 \\
\hline 50 & 50 & 50 & 50 & N/A \\
\hline 68 & 68 & 60 & N/A & N/A \\
\hline 44 & 21 & 15 & 43 & 43 \\
\hline 36 & 37 & 23 & 34 & 25 \\
\hline$N / A$ & N/A & N/A & 2 & 1 \\
\hline 96 & 66 & 71 & 90 & 85 \\
\hline
\end{tabular}

\section{5}

94

99

99

85

N/A

100

85

84

88

90

105

01

WEIGHTED AVERAGE PRICE (\$MMMBTU) OFIMPORTS

\begin{tabular}{|c|c|c|c|c|}
\hline $\begin{array}{c}1994 \\
\text { qtr } \\
\# 1\end{array}$ & $\begin{array}{c}1994 \\
\text { qtr } \\
\# 2\end{array}$ & $\begin{array}{c}1994 \\
\text { qtr } \\
\# 3\end{array}$ & $\begin{array}{c}1994 \\
\text { qtr } \\
\# 4\end{array}$ & $\begin{array}{c}1995 \\
\text { qtr } \\
\# 1\end{array}$ \\
\hline 3.01 & 2.64 & 2.44 & 2.21 & 2.05 \\
\hline 2.92 & 2.93 & 2.86 & 2.97 & 2.97 \\
\hline 1.66 & 1.55 & 1.49 & 1.43 & 1.35 \\
\hline 1.81 & 1.59 & 1.51 & 1.38 & 1.33 \\
\hline 1.11 & 0.93 & 0.83 & N/A & $\mathrm{N} / \mathrm{A}$ \\
\hline 1.13 & 0.93 & 0.79 & 0.67 & 0.48 \\
\hline 1.11 & 0.93 & 0.80 & 0.67 & 0.53 \\
\hline 2.97 & 2.97 & 2.97 & 3.05 & 3.09 \\
\hline N/A & N/A & N/A & 2.25 & 2.11 \\
\hline N/A & N/A & N/A & 2.27 & 2.15 \\
\hline N/A & N/A & N/A & 2.27 & 2.16 \\
\hline 2.43 & 2.30 & 2.43 & 2.21 & 2.35 \\
\hline N/A & $N / A$ & N/A & 3.00 & 3.01 \\
\hline 2.97 & 2.72 & 2.64 & 2.44 & 2.28 \\
\hline 1.91 & 1.76 & 1.44 & 1.12 & 1.06 \\
\hline 1.91 & 1.76 & 1.44 & 1.16 & 1.08 \\
\hline 1.74 & 1.72 & 1.34 & 1.07 & 1.27 \\
\hline 1.91 & 1.76 & 1.44 & 1.19 & 1.06 \\
\hline 2.69 & 2.18 & 2.21 & 2.25 & 2.32 \\
\hline 2.69 & 2.18 & 2.15 & 2.25 & 2.32 \\
\hline 2.86 & 2.18 & 2.23 & 2.25 & 2.32 \\
\hline 2.71 & 2.18 & 2.21 & 2.27 & 2.32 \\
\hline 2.73 & 2.18 & 2.17 & 2.16 & $N / A$ \\
\hline 2.95 & 2.54 & 2.58 & 2.55 & 2.37 \\
\hline 2.10 & 2.10 & 2.10 & 2.13 & 2.26 \\
\hline 1.66 & 1.67 & 1.69 & 1.77 & 1.80 \\
\hline N/A & 2.00 & 2.00 & 2.00 & 2.10 \\
\hline N/A & 2.15 & 2.15 & 2.14 & 2.22 \\
\hline N/A & 1.48 & 1.52 & 1.43 & 1.21 \\
\hline 3.10 & 2.77 & 2.52 & 2.19 & $N / A$ \\
\hline 2.80 & 2.47 & 2.42 & N/A & $\mathrm{N} / \mathrm{A}$ \\
\hline 1.45 & 1.41 & 1.43 & 1.44 & 1.39 \\
\hline 1.36 & 1.36 & 1.36 & 1.36 & 1.36 \\
\hline N/A & N/A & N/A & 2.56 & 2.03 \\
\hline 1.90 & 1.90 & 1.90 & 1.90 & 1.97 \\
\hline
\end{tabular}

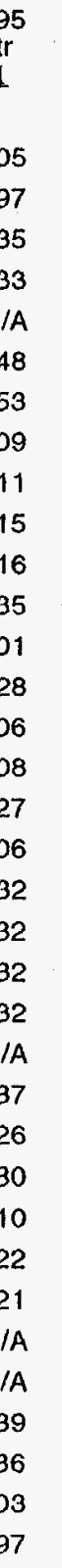

ROCHESTER GAS AND ELECTRIC CORPORATION/Westcoast Gas Services SALMON RESOURCES LTD./Shell Canada Limited SALMON RESOURCES LTD.-ENRON GAS MARKET//Shell Canada Limited SALMON RESOURCES LTD.-MIDWEST GAS/Shell Canada Limited SAN DIEGO GAS \& ELECTRIC COMPANY/Bow Valley Industries Ltd. SAN DIEGO GAS \& ELECTRIC COMPANY/Canadian Hunter Marketing Ltd. SAN DIEGO GAS \& ELECTRIC COMPANY/Husky Oil Operations Lid. SARANAC POWER PARTNERS, L.P./Shell Canada Limited SELKIRK COGEN PARTNERS, L.P./ATCOR LId.

SELKIRK COGEN PARTNERS, L.P./Imperial Oil Resources Limited SELKIRK COGEN PARTNERS, L.P./PanCanadian Petroleum Ltd. SELKIRK COGEN PARTNERS, L.P.IParamount Resources Ltd. SITHE/NDEPENDENCE POWER PARTNERS, L.P.IEnron Power Services inc. SOUTHEASTERN MICHIGAN GAS COMPANY/WGM LId.

SOUTHERN CALIFORNIA EDISON COMPANY/AEC Oil \& Gas Company

SOUTHERN CALIFORNIA EDISON COMPANY/Imperial Oil Resources Limited SOUTHERN CALIFORNIA EDISON COMPANY/Shell Canada Limited SOUTHERN CALIFORNIA EDISON COMPANY/WGM Ltd. SOUTHERN CONNECTICUT GAS/Alberta NE Gas (AEC Oil \& Gas Company) SOUTHERN CONNECTICUT GAS/Alberta Northeast Gas (Atcor) SOUTHERN CONNECTICUT GAS/Alberta Northeast Gas (ProGas) SOUTHERN CONNECTICUT GAS/Alberta Northeast Gas (WGM Ltd. 1) SOUTHERN CONNECTICUT GAS/Alberta Northeast Gas (WGM Ltd. 2) ST. LAWRENCE GAS COMPANY INC./Niagara Gas Transmission Ltd. SUMAS COGENERATION COMPANY, L.P.JENCO Gas, Lid.

SUMAS COGENERATION COMPANY, L.P.Nestcoast Gas Services TENASKA GAS COMPANY/Husky Oil Operations Litd.

TENASKA GAS COMPANY/Petro-Canada Ltd.

TENASKA GAS COMPANY/Talisman Energy Inc.

TENNESSEE GAS PIPELINE COMPANY/KannGaz Producers Ltd.

TENNESSEE GAS PIPELINE COMPANY/TransCanada Pipelines Limited

THE MONTANA POWER COMPANY/Canadian-Montana Pipeline Company

THE MONTANA POWER COMPANY/Canadian-Montana Pipeline Company

THE U.S. GENERAL SERVICES ADMINISTRATIO/Chiel Mountain Natural Gas Coop. N/A

TM STAR FUEL COMPANY/CanWest Gas Supply 
Page - 6

\section{LONG-TERM IMPORTS}

ACTUAL IMPORTS AS A PERCENT OF AUTHORIZED VOLUMES

\section{IMPORTERIEXPORTER}

TRANSCO ENERGY MARKETING COMPANY/Canstates Gas Marketing TRANSCO ENERGY MARKETING COMPANY-BG\&E/Canstates Gas Marketing TRANSCO ENERGY MARKETING COMPANY-LILCO/Canstates Gas Marketing TRANSCO ENERGY MARKETING COMPANY-PSE\&G/Canstates Gas Marketing UTILICORP UNITED, INC./ProGas Limited

UTILICORP UNITED, INC./WGM Ltd.

VALLEY GAS COMPANY/Alberta Northeast Gas (WGM Ltd. 1)

VERMONT GAS SYSTEMS INC.MGM LId.

WASHINGTON NATURAL GAS COMPANYIAmoco Canada

WASHINGTON NATURAL GAS COMPANYIMobil Oil Canada

WASHINGTON NATURAL GAS COMPANY/Poco Petroleums Ltd.

WASHINGTON NATURAL GAS COMPANYMestcoast Gas Services

WASHINGTON NATURAL GAS COMPANYM Westcoast Gas Services

WASHINGTON WATER POWER COMPANYIAEC OII \& Gas Company

WASHINGTON WATER POWER COMPANY/Amerada Hess Canada Ltd.

WASHINGTON WATER POWER COMPANY/PanCanadian Petroleum Limited

WASHINGTON WATER POWER COMPANYMestcoast Gas Services

WESTCOAST GAS SERVICES INC. - CIBOLA COMUestcoast Gas

WESTCOAST GAS SERVICES INC. - INTERSTAT/Westcoast GaS

WESTCOAST GAS SERVICES INC. - METRO. UT/Westcoast Gas

WESTCOAST GAS SERVICES INC. - MIDWEST G/Westcoast Gas

WESTCOAST GAS SERVICES INC. - N'WESTERN/Westcoast Gas

WESTCOAST GAS SERVICES INC. - WISCONSIN/Westcoast Gas

WESTERN GAS MARKETING INC.WGM LId.

WISCONSIN FUEL \& LIGHT COMPANY/Progas Limited

WISCONSIN FUEL \& LIGHT COMPANYMGM LId.

WISCONSIN GAS COMPANY/ProGas Limited (I)

WISCONSIN GAS COMPANYIProGas Limited (II)

WISCONSIN GAS COMPANYNGM LId.

WISCONSIN GAS COMPANYNGM Ltd.

WISCONSIN NATURAL GAS COMPANY/Progas Limited

WISCONSIN NATURAL GAS COMPANYNGM Ltd.

WISCONSIN POWER \& LIGHT COMPANY/ProGas Limited

WISCONSIN POWER \& LIGHT COMPANYIWGM Ltd.

WISCONSIN POWER \& LIGHT COMPANY/WGM LId.

\begin{tabular}{|c|c|c|c|c|}
\hline $\begin{array}{c}1994 \\
q t r \\
\# 1\end{array}$ & $\begin{array}{c}1994 \\
q u r \\
\# 2\end{array}$ & $\begin{array}{c}1994 \\
\text { qtr } \\
\# 3\end{array}$ & $\begin{array}{c}1994 \\
\text { qtr } \\
\# 4\end{array}$ & $\begin{array}{r}199 \\
\text { qtr } \\
\# 1\end{array}$ \\
\hline 100 & 99 & 99 & 98 & 4 \\
\hline 38 & 22 & 34 & 41 & a \\
\hline 99 & 6 & N/A & 89 & 97 \\
\hline N/A & 19 & 13 & 30 & 8 \\
\hline 72 & 99 & 99 & 99 & 95 \\
\hline 73 & 101 & 100 & 101 & 100 \\
\hline 99 & 99 & 99 & 99 & 99 \\
\hline 94 & 46 & 31 & 68 & 89 \\
\hline 101 & 100 & 101 & 81 & 104 \\
\hline 97 & 82 & 49 & 86 & 36 \\
\hline 101 & 100 & 99 & 101 & 104 \\
\hline 101 & 101 & 65 & 101 & 104 \\
\hline 26 & 9 & 40 & 71 & 42 \\
\hline 44 & $N / A$ & N/A & 64 & 45 \\
\hline 64 & 64 & 64 & 69 & 71 \\
\hline 50 & 37 & 37 & 56 & 58 \\
\hline 76 & 33 & 67 & 79 & 79 \\
\hline N/A & N/A & N/A & 101 & 101 \\
\hline N/A & N/A & $N / A$ & 107 & 98 \\
\hline N/A & N/A & N/A & 80 & 72 \\
\hline N/A & N/A & N/A & 101 & 93 \\
\hline N/A & $N / A$ & $N / A$ & 105 & 105 \\
\hline N/A & N/A & N/A & 101 & 86 \\
\hline 85 & 55 & 61 & 60 & N/A \\
\hline 100 & 99 & 99 & 100 & 100 \\
\hline 98 & 99 & 99 & 89 & 100 \\
\hline N/A & 97 & 98 & 98 & 99 \\
\hline N/A & 100 & 99 & 100 & 100 \\
\hline N/A & 99 & 99 & 99 & 92 \\
\hline N/A & N/A & N/A & 100 & 85 \\
\hline 96 & 99 & 98 & 98 & 100 \\
\hline 97 & 100 & 99 & 100 & 91 \\
\hline 99 & 99 & 99 & 100 & 100 \\
\hline 98 & 97 & 98 & 98 & 98 \\
\hline 98 & 98 & 98 & 99 & 98 \\
\hline
\end{tabular}

WEIGHTED AVERAGE PRICE (\$MMBTU) OF IMPORTS

\begin{tabular}{|c|c|c|c|c|}
\hline $\begin{array}{c}1994 \\
\text { qtr } \\
\# 1\end{array}$ & $\begin{array}{c}1994 \\
\mathrm{qtr} \\
\mathbb{1 1 2}\end{array}$ & $\begin{array}{c}1994 \\
\text { qtr } \\
\# 3\end{array}$ & $\begin{array}{c}1994 \\
\text { qtr } \\
\text { 帅 }\end{array}$ & $\begin{array}{c}1995 \\
\text { qtr } \\
\text { 亗 }\end{array}$ \\
\hline 3.10 & 2.61 & 2.32 & 2.03 & 2.07 \\
\hline 1.75 & 2.61 & 2.48 & 2.24 & 2.04 \\
\hline 1.82 & 2.90 & N/A & 2.46 & 2.40 \\
\hline N/A & 2.93 & 2.41 & 2.56 & 2.36 \\
\hline 2.43 & 2.02 & 1.81 & 1.71 & 1.64 \\
\hline 2.45 & 2.03 & 1.81 & 1.70 & 1.60 \\
\hline 2.71 & 2.18 & 2.21 & 2.25 & 2.32 \\
\hline 2.90 & 3.72 & 4.68 & 2.73 & 2.13 \\
\hline 2.15 & 1.85 & 1.85 & 1.76 & 1.20 \\
\hline 2.35 & 2.20 & 2.80 & 2.14 & 3.57 \\
\hline 2.27 & 1.95 & 1.62 & 1.58 & 1.52 \\
\hline 2.08 & 2.10 & 2.42 & 1.68 & 1.17 \\
\hline 2.60 & 3.77 & 1.94 & 1.69 & 1.83 \\
\hline 2.32 & N/A & N/A & 1.98 & 1.98 \\
\hline 2.00 & 1.75 & 1.53 & 1.18 & 1.02 \\
\hline 1.96 & 1.73 & 1.97 & 1.75 & 1.67 \\
\hline 2.25 & 2.21 & 1.68 & 1.63 & 1.43 \\
\hline N/A & N/A & $N / A$ & 1.40 & 1.17 \\
\hline N/A & N/A & N/A & 1.64 & 1.62 \\
\hline N/A & N/A & N/A & 1.97 & 1.91 \\
\hline N/A & N/A & N/A & 1.64 & 1.53 \\
\hline N/A & N/A & N/A & 1.94 & 1.50 \\
\hline N/A & N/A & N/A & 1.77 & 1.65 \\
\hline 2.46 & 2.21 & 2.10 & 1.84 & N/A \\
\hline 2.38 & 2.04 & 1.82 & 1.71 & 1.61 \\
\hline 2.25 & 2.07 & 1.87 & 1.82 & 1.64 \\
\hline N/A & 2.05 & 1.83 & 1.72 & 1.61 \\
\hline N/A & 2.04 & 1.82 & 1.71 & 1.62 \\
\hline N/A & 2.06 & 1.87 & 1.77 & 1.83 \\
\hline N/A & $N / A$ & N/A & 1.84 & 1.74 \\
\hline 2.40 & 2.04 & 1.83 & 1.73 & 1.63 \\
\hline 2.36 & 2.06 & 1.86 & 1.76 & 1.73 \\
\hline 2.36 & 2.00 & 1.79 & 1.69 & 1.62 \\
\hline 2.39 & 2.05 & 1.89 & 1.72 & \\
\hline
\end{tabular}




\section{LONG-TERM IMPORTS}

\section{ACTUAL IMPORTS AS A PERCENT OF AUTHORIZED VOLUMES}

$\begin{array}{ccccc}1994 & 1994 & 1994 & 1994 & 1995 \\ \text { qtr } & \text { qtr } & \text { qtr } & \text { qtr } & \text { qtr } \\ \# 1 & \# 2 & \# 3 & \# 4 & \# 1\end{array}$

WISCONSIN PUBLIC SERVICE CORPORATION/PrOGas Limited WISCONSIN PUBLIC SERVICE CORPORATION/ProGas Limited WISCONSIN PUBLIC SEPVICE CORPORATIONNGM LId

YANKEE GAS SERVICES CO./Alberta NE Gas (AEC Oil \& Gas Company)

YANKEE GAS SERVICES CO./Alberta Northeast Gas (Atcor)

YANKEE GAS SERVICES CO./Alberta Northeast Gas (ProGas)

YANKEE GAS SERVICES CO./Alberta Northeast Gas (WGM Lid. 1)

YANKEE GAS SERVICES CO./Alberta Northeast Gas (WGM Litd. 2)

$\begin{array}{lllll}100 & 100 & 100 & 100 & 100\end{array}$

N/A $\quad 96 \quad 99 \quad 99 \quad 100$

$101 \quad 101$

$100 \quad 100$

100100

$100 \quad 100$
$99 \quad 99 \quad 100$

$101 \quad 101 \quad 101$

$100 \quad 100 \quad 100$

$100 \quad 100 \quad 100$

$100 \quad 100$

$100 \quad 100 \quad 100$

WEIGHTED AVERAGE PRICE (\$MMBTU) OF IMPORTS

\begin{tabular}{|c|c|c|c|c|}
\hline $\begin{array}{c}1994 \\
\text { qtr } \\
\mathbb{1 1}\end{array}$ & $\begin{array}{c}1994 \\
\text { qtr } \\
\# 2\end{array}$ & $\begin{array}{c}1994 \\
q 4 r \\
\# 3\end{array}$ & $\begin{array}{c}1994 \\
\text { qtr } \\
\# 4\end{array}$ & $\begin{array}{c}1995 \\
\text { qtr } \\
\# 1\end{array}$ \\
\hline 2.38 & 2.03 & 1.81 & 1.72 & 1.63 \\
\hline $\mathrm{N} / \mathrm{A}$ & 2.00 & 1.81 & 1.71 & 1.64 \\
\hline 2.35 & 2.06 & 1.87 & 1.77 & 1.68 \\
\hline 2.69 & 2.18 & 2.21 & 2.25 & 2.32 \\
\hline 2.69 & 2.18 & 2.15 & 2.25 & 2.32 \\
\hline 2.86 & 2.18 & 2.23 & 2.25 & 2.32 \\
\hline 2.71 & 2.18 & 2.21 & 2.25 & 2.32 \\
\hline 2.73 & 2.18 & 2.17 & 2.25 & 2.32 \\
\hline
\end{tabular}


Long-Term Importer

AG-ENERGY, L.P.

(Home Oil Company Limited)

AVG Daily Quantity

Total Price (Waddington, NY)

Commodity Component

Demand Component

Reservation Fee Component

BOSTON GAS COMPANY

(Alberta Northeast Gas (WGM Ltd. 1))

AVG Daily Quantity

Total Price (Waddington, NY)

Commodity Component

Demand Component

Reservation Fee Component

BOSTON GAS COMPANY

(Esso Resources Canada Limited)

AVG Daily Quantity

Total Price (Waddington, NY)

Commodity Componen

Demand Component

Reservation Fee Component

BOUNDARY GAS, INC.

(TransCanada Pipelines Limited)

AVG Daily Quantity

Total Price (Niagara Falls, NY)

Commodity Component

Demand Component

Reservation Fee Component

BROOKLYN UNION GAS COMPANY

(Alberta NE Gas (AEC Oil \& Gas Company))

AVG Daily Quantity

Total Price (Waddington, NY)

Commodity Compone

Demand Component

Reservation Fee Component

\section{VOLUME AND PRICE REPORT}

Long Term Imports

Volumes (MMct/d) \& Prices (\$MMBTU) of Natural

Gas Imported During the Past 12 Months 04/01/94 - 03/31/95

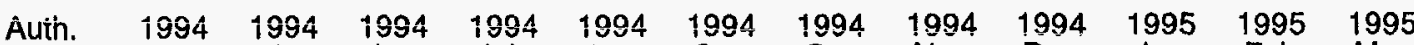

Vols. Apr May. Jun Jul Aug. Sep. Oct Nov Dec. Jan. Feb. Mar.

17.5

$\begin{array}{llllllllllll}12.0 & 14.6 & 15.2 & 14.1 & 15.0 & 12.5 & 14.6 & 15.8 & 16.1 & 15.2 & 16.5 & 16.2 \\ \frac{2.27}{1.36} & \frac{2.27}{1.36} & \frac{2.27}{1.36} & \frac{2.27}{1.36} & \frac{2.27}{1.36} & \frac{2.27}{1.36} & \frac{2.25}{1.36} & \frac{2.29}{1.40} & \frac{2.29}{1.40} & \frac{2.30}{1.40} & \frac{2.23}{1.40} & \frac{2.30}{1.40} \\ 0.91 & 0.91 & 0.91 & 0.91 & 0.91 & 0.91 & 0.89 & 0.89 & 0.89 & 0.90 & 0.83 & 0.90 \\ 0.00 & 0.00 & 0.00 & 0.00 & 0.00 & 0.00 & 0.00 & 0.00 & 0.00 & 0.00 & 0.00 & 0.00\end{array}$

8.6

\begin{tabular}{rrrrrrrrrrrr}
8.6 & 8.6 & 8.6 & 8.6 & 8.6 & 8.6 & 8.6 & 8.6 & 8.6 & 8.6 & 8.6 & 8.6 \\
2.24 & 2.17 & 2.12 & 2.25 & 2.23 & 2.14 & 1.96 & 2.36 & 2.42 & 2.38 & 2.31 & $\frac{2.26}{1.42}$ \\
\hline 1.37 & 1.30 & 1.25 & 1.35 & 1.31 & 1.23 & 1.04 & 1.45 & 1.52 & $\frac{1.47}{1.42}$ & 1.36 \\
0.87 & 0.87 & 0.87 & 0.90 & 0.92 & 0.91 & 0.92 & 0.91 & 0.90 & 0.91 & 0.89 & 0.90 \\
0.00 & 0.00 & 0.00 & 0.00 & 0.00 & 0.00 & 0.00 & 0.00 & 0.00 & 0.00 & 0.00 & 0.00
\end{tabular}

35.0

\begin{tabular}{|c|c|c|c|c|c|c|c|c|c|c|c|}
\hline $\begin{array}{l}35.0 \\
2.57 \\
1.68 \\
0.89\end{array}$ & $\begin{array}{l}35.0 \\
2.60 \\
1.74 \\
0.86 \\
0.00\end{array}$ & $\begin{array}{l}35.0 \\
2.42 \\
1.53 \\
0.89 \\
0.00\end{array}$ & $\begin{array}{l}35.0 \\
2.54 \\
1.66 \\
0.88 \\
0.00\end{array}$ & $\begin{array}{l}35.0 \\
2.42 \\
1.54 \\
0.88\end{array}$ & $\begin{array}{l}35.0 \\
2.23 \\
1.30 \\
0.93 \\
0.00\end{array}$ & $\begin{array}{l}35.0 \\
2.13 \\
1.23 \\
0.90 \\
0.00\end{array}$ & $\begin{array}{l}20.0 \\
3.29 \\
1.68 \\
1.61 \\
0.00\end{array}$ & $\begin{array}{l}35.0 \\
2.56 \\
1.69 \\
0.87 \\
0.00\end{array}$ & $\begin{array}{l}35.0 \\
\frac{2.50}{1.62} \\
0.88 \\
0.00\end{array}$ & $\begin{array}{l}35.0 \\
2.44 \\
1.46 \\
0.98 \\
0.00\end{array}$ & \\
\hline
\end{tabular}

92.5

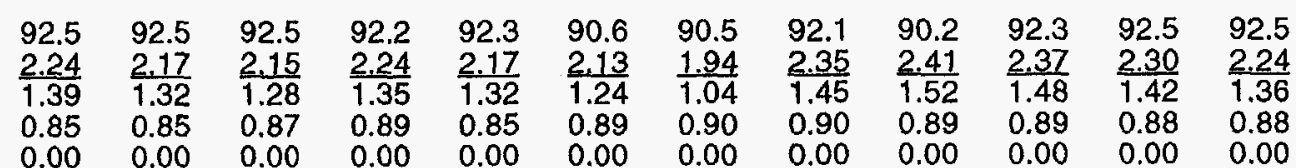

4.0

$\begin{array}{rrr}4.0 & 4.0 & 4.0 \\ \frac{2.17}{1.31} & \frac{2.24}{1.39} & \frac{2.12}{1.27} \\ 0.86 & 0.85 & 0.85 \\ 0.00 & 0.00 & 0.00\end{array}$

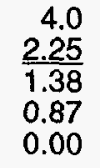

4.0
2.23
1.35
0.88
0.00

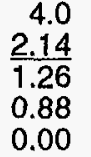

\begin{tabular}{l}
2.14 \\
\hline 1.26 \\
0.88 \\
0.00
\end{tabular}
4.0
2.36
1.48
0.88
0.00
4.0
2.42
1.50
0.92
0.00

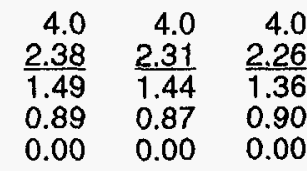

1095 TOTAL Mct

$1,434,770$

$1,434,770$

774,000

774,000

$3,150,081$

$3,150,081$

$8,319,949$

$8,319,949$

355,590

355,590 
Long-Term Importer

BROOKLYN UNION GAS COMPANY (Alberta Northeast Gas (Atcor))

AVG Daily Quantity

Total Price (Waddington. NY)

Commodity Component

Demand Component

Reservation Fee Component

BROOKLYN UNION GAS COMPANY

(Alberta Northeast Gas (ProGas))

AVG Daily Quantity

Total Price (Waddington. NY)

Commodity Component

Demand Component

Reservation Fee Component

BROOKLYN UNION GAS COMPANY

(Alberta Northeast Gas (WGM Ltd. 1))

AVG Daily Quantity

Total Price (Waddington, NY)

Commodity Component

Demand Component

Reservation Fee Component

BROOKLYN UNION GAS COMPANY

(Alberta Northeast Gas (WGM Ltd. 2))

AVG Daily Quantity

Total Price (Waddington. NY)

Commodity Component

Demand Component

Reservation Fee Component

CASCADE NATURAL GAS CORPORATION (Mobil Oil Canada, Ltd.)

AVG Daily Quantity

Total Price (Sumas, Washington)

Commodity Component

Demand Component

Reservation Fee Component

\section{VOLUME AND PRICE REPORT}

\section{Long Term Imports}

Volumes (MMct/d) \& Prices ( $\$ M M M B T U$ ) of Natural

Gas Imported During the Past 12 Months 04/01/94 - 03/31/95

Vols.

19941994

8.2
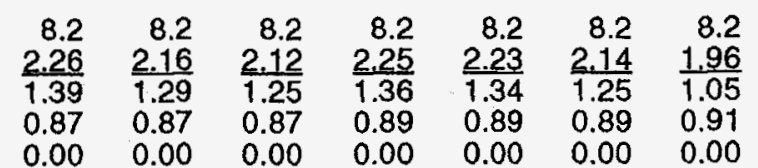

8.2
2.37
1.46
0.91
0.00

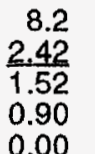

\begin{tabular}{r}
8.2 \\
2.39 \\
\hline 1.50 \\
0.89 \\
0.00
\end{tabular}

\begin{tabular}{rr}
8.2 & 8.2 \\
2.31 & 2.26 \\
\hline 1.42 & 1.36 \\
0.89 & 0.90 \\
0.00 & 0.00
\end{tabular}

15.8

$\begin{array}{lll}15.8 & 15.8 & 15.8 \\ \frac{2.24}{1.32} & \frac{2.17}{1.26} & 2.1 \\ 0.92 & 0.91 & 0.91 \\ 0.00 & 0.00 & 0.00\end{array}$

15.8
2.12
1.21
0.91
0.00

15.7
2.30
1.35
0.95
0.00

15.8

$\begin{array}{ll}15.8 & 15.8\end{array}$

15.8

15.8
2.42

1.31

24.0

$\begin{array}{ll}24.0 & 24.0 \\ 2.24 & 2.17 \\ 1.37 & 1.30 \\ 0.87 & 0.87 \\ 0.00 & 0.00\end{array}$

$\begin{array}{ll}24.0 & 2 \\ 2.17 & 2 \\ 1.30 & 1 \\ 0.87 & 0.07 \\ 0.00 & 0.00\end{array}$

24.0
2.12
1.25
0.87
0.00

24.0
2.25
1.35
0.90
0.00

22.5

$\frac{2.23}{1.31}$

0.92
0.00

\begin{tabular}{l}
24.0 \\
2.14 \\
\hline
\end{tabular}

$\frac{2.14}{1.23}$
0.91

0.91
0.00

\begin{tabular}{l}
24.0 \\
1.96 \\
\hline 1.04 \\
0.92 \\
0.00
\end{tabular}

\begin{tabular}{l}
24.0 \\
2.36 \\
\hline
\end{tabular}

$\frac{2.36}{1.45}$

0.91
0.00

24.0
2.42
1.52

1.52

0.90
0.00

23.1
$\frac{2.38}{1.47}$

1.47
0.91

0.91
0.00

24.0

$\frac{2.31}{1.42}$

0.89

18.0

$\begin{array}{lll}18.0 & 18.0 & 18 . \\ 2.24 & 2.17 & 2.1 \\ 1.37 & 1.30 & 1.25 \\ 0.87 & 0.87 & 0.87 \\ 0.00 & 0.00 & 0.00\end{array}$

\begin{tabular}{ll}
18.0 & 18.0 \\
2.17 & 2.2 \\
\hline 1.30 & 1.3 \\
0.87 & 0.9 \\
0.00 & 0.0
\end{tabular}

$\begin{array}{ll}8.0 & 18.0 \\ .22 & 2.15 \\ .32 & 1.25 \\ .90 & 0.90 \\ .00 & 0.00\end{array}$

18.0

$\frac{1.96}{1.04}$

18.0

18.0
2.36
1.45

18.0
2.42

18.0
2.38

18.0

0.92
0.00

0.91
0.00

0.90
0.00

$\frac{2.38}{1.47}$

0.91
0.00

$\frac{2.31}{1.42}$

1.42
0.89

0.00

12.0

\begin{tabular}{rrr}
8.9 & 11.5 & 11.5 \\
2.28 & 2.26 & 2.02 \\
\hline 1.62 & 1.60 & 1.38 \\
0.52 & 0.52 & 0.52 \\
0.14 & 0.14 & 0.12
\end{tabular}

11.3
2.12
1.47
0.52
0.13

\begin{tabular}{l}
11.4 \\
2.10 \\
\hline 1.45 \\
0.52 \\
0.13
\end{tabular}

\begin{tabular}{l}
11.5 \\
2.01 \\
\hline 1.36 \\
0.53 \\
0.12
\end{tabular}
11.5
1.95
1.30
0.5
0.1

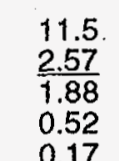

11.5
2.57
1.88
0.52
0.17
11.5
2.58
1.88
0.53
0.17

\begin{tabular}{l}
11.5 \\
2.59 \\
\hline 1.88 \\
0.54 \\
0.17
\end{tabular}
18.0

$\frac{2.26}{1.36}$

0.90

0.00

1Q95

IOTAL MC

738,270

738,270

TOTAL MC

$1,425,330$

$1,425,330$

$\frac{2.26}{1.27}$

0.99

0.00

24.0

$\frac{2.26}{1.36}$

0.90
0.00

$2,133,340$

$2,133,340$

$1,620,000$

$1,620,000$

\begin{tabular}{l}
11.5 \\
1.44 \\
\hline 0.83 \\
0.54 \\
0.07
\end{tabular}

$1,034,959$

$1,034,959$ 
Long-Term Importer

CASCADE NATURAL GAS CORPORATION (Westcoast Gas Services)

AVG Daily Quantity

Total Price (Eastport, Idaho)

Commodity Component

Demand Component

Reservation Fee Component

CASCADE NATURAL GAS CORPORATION (Westcoast Gas Services)

AVG Daily Quantity

Total Price (Sumas, Washington)

Commodity Component

Demand Component

Reservation Fee Component

CASCADE NATURAL GAS CORPORATION (Westcoast Gas Services)

AVG Daily Quantity

Total Price (Sumas, Washington)

Commodity Component

Demand Component

Reservation Fee Component

CENTRAL HUDSON COMPANY

(Alberta NE Gas (AEC Oil \& Gas Company))

AVG Daily Quantity

Total Price (Waddington, NY)

Commodity Component

Demand Component

Reservation Fee Component

CENTRAL HUDSON COMPANY

(Alberta Northeast Gas (Atcor))

AVG Daily Quantity

Total Price (Waddington, NY)

Commodity Component

Demand Component

Reservation Fee Component

\section{VOLUME AND PRICE REPORT}

\section{Long Term Imports}

Volumes (MMcf/d) \& Prices (\$/MMBTU) of Natural

Gas Imported During the Past 12 Months 04/01/94 - 03/31/95

Auth.

$1994 \quad 1994 \quad 1904$

Vols,

Apr.

33.2

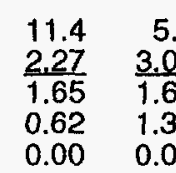

10.0

\begin{tabular}{rrr}
9.8 & 9.8 & 9.8 \\
1.89 & $\frac{1.86}{1.43}$ & $\frac{1.67}{1.22}$ \\
\hline 1.45 & 1.43 & 0.45 \\
0.44 & 0.43 & 0.45
\end{tabular}

$\begin{array}{lll}0.44 & 0.43 & 0.45\end{array}$

4.9

\begin{tabular}{rr}
4.9 & 4.9 \\
2.18 & 2.17 \\
\hline 1.70 & 1.69 \\
0.18 & 0.18
\end{tabular}

$\begin{array}{ll}0.30 & 0.30\end{array}$

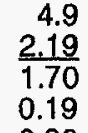

$\frac{2.19}{1.70}$

0.19
0.30

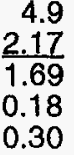

\begin{tabular}{rr}
4.9 \\
2.17 \\
\hline 1.69 \\
0.18 \\
0.30
\end{tabular}
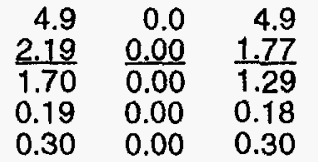

4.7
1.78
1.30
0.18
0.30

\begin{tabular}{r}
4.9 \\
1.75 \\
\hline 1.28 \\
0.18 \\
0.29
\end{tabular}

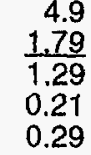

$\begin{array}{r}4.9 \\ 1.75 \\ \hline 1.27\end{array}$

$\frac{1.75}{1.27}$ 0.19
0.29

0.1

$\begin{array}{ll}0.1 & 0.1 \\ 2.17 & 2.2 \\ 1.31 & 1.39 \\ 0.86 & 0.85 \\ 0.00 & 0.00\end{array}$

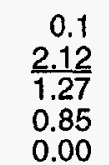

0.1
2.25
1.38
0.87
0.00

0.1
2.23
1.35
0.88

0.1
2.14
1.26
0.88

\begin{tabular}{r}
0.1 \\
1.96 \\
\hline 1.07 \\
0.89 \\
0.00
\end{tabular}

\begin{tabular}{r}
0.1 \\
2.36 \\
\hline 1.48 \\
0.88 \\
0.00
\end{tabular}

0.1
2.42
1.50
0.92

$\begin{array}{r}0.1 \\ 2.38 \\ \hline 1.49\end{array}$

0.89

0.1
2.31
1.44

$\frac{2.31}{1.44}$

$0.87 \quad 0.90$

0.0

3.0

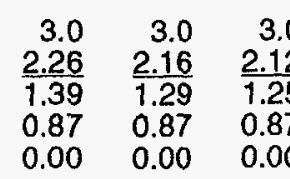

$\begin{array}{lll}0.00 & 0.00 & 0.00\end{array}$

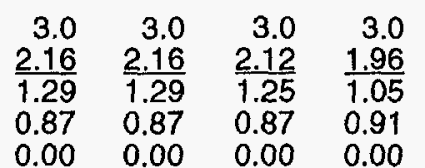

\begin{tabular}{rrrr}
3.0 & 3.0 & 3.0 & 3.0 \\
2.16 & 2.16 & 2.12 & 1.96 \\
\hline 1.29 & 1.29 & 1.25 & 1.05 \\
0.87 & 0.87 & 0.87 & 0.91 \\
0.00 & 0.00 & 0.00 & 0.00
\end{tabular}

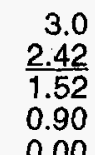

3.0
2.39
1.50
0.89
0.00

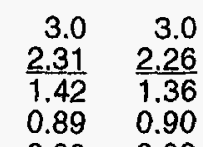

TOTÁ

TOTAL Micf

$1,780,687$

882,231

882,231

441,610

441,610

8,640

8,640

268,560

268,560 


\section{Long-Term Importer}

CENTRAL HUDSON COMPANY (Alberta Northeast Gas (ProGas)) AVG Daily Quantity

Total Price (Waddington, NY)

Commodity Component

Demand Component

Reservation Fee Component

CENTRAL HUDSON COMPANY

(Alberta Northeast Gas (WGM Ltd. 1))

AVG Daily Quantity

Total Price (Waddington. NY)

Commodity Component

Demand Component

Reservation Fee Component

CENTRAL HUDSON COMPANY

(Alberta Northeast Gas (WGM Ltd. 2))

AVG Daily Quantity

Total Price (Waddington. NY)

Commodity Component

Demand Component

Reservation Fee Component

CITY OF BURBANK, CALIFORNIA

(Westcoast Gas Services)

AVG Daily Quantity

Total Price (Eastport, Idaho)

Commodity Component

Demand Component

Reservation Fee Component

CITY OF GLENDALE CALIFOANIA

(Westcoast Gas Services)

AVG Daily Quantity

Total Price (Eastport, Idaho)

Commodity Component

Demand Component

Reservation Fee Component

\section{VOLUME AND PRICE REPORT}

\section{Long Term Imports}

Volumes (MMct/d) \& Prices ( $\$ M M B T U)$ of Natural Gas Imported During the Past 12 Months 04/01/94 - 03/31/95

Auth. $\quad \begin{array}{llllllllllll}1994 & 1994 & 1994 & 1994 & 1994 & 1994 & 1994 & 1994 & 1994 & 1995 & 1995 & 1995\end{array}$

Vols. Apr. May Jun Jul. Aug. Sep. Qct Nov Dec. Jan. Feb. Mar.

$$
\begin{array}{ll}
1.3 \\
\frac{2.24}{1.32} \\
0.92 \\
0.00
\end{array}
$$

$\begin{array}{ll}1.3 & 1.3 \\ \frac{2.17}{1.26} & 2.12 \\ 0.91 & 0.21 \\ 0.00 & 0.01\end{array}$
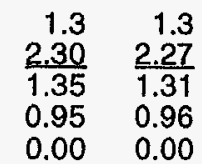
1.31

0.00

0.00
14.1

\begin{tabular}{|c|c|c|}
\hline $\begin{array}{l}14.1 \\
2.24 \\
1.37 \\
0.87 \\
0.00\end{array}$ & $\begin{array}{l}14.1 \\
2.17 \\
1.30 \\
0.87 \\
0.00\end{array}$ & $\begin{array}{l}14.1 \\
2.12 \\
1.25 \\
0.87 \\
0.00\end{array}$ \\
\hline
\end{tabular}

1.5

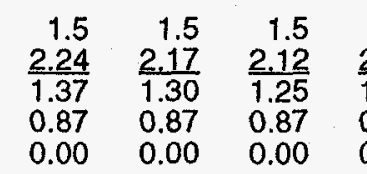

1.5
2.17
1.30
0.87
0.00

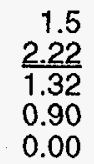

\begin{tabular}{r}
1.5 \\
2.12 \\
\hline 1.25 \\
0.87 \\
0.00
\end{tabular}

1.5
1.96
1.04
0.92
0.00

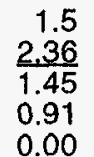

1.5
2.42
1.52
0.90
0.00

1.5
2.38
1.47
0.91
0.00

4.8

$$
\begin{array}{ll}
4.7 & 4.7 \\
1.72 & 1.7 \\
\hline 1.44 & 1.44 \\
0.28 & 0.27 \\
0.00 & 0.00
\end{array}
$$

4.7
1.72
1.44
0.28
0.00

4.7
1.84
1.57
0.27
0.00

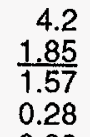

$\begin{array}{r}4.2 \\ 1.85 \\ \hline 1.57\end{array}$

4.1

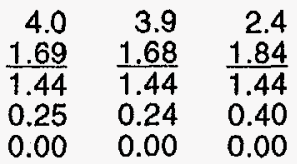

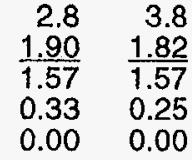

0.00 $\begin{array}{ll}1.57 & 1.17\end{array}$

$\begin{array}{ll}0.00 & 0.00\end{array}$

$\frac{1.85}{1.57}$

0.28
0.00

$\begin{array}{r}4.7 \\ 1.84 \\ \hline 1.57\end{array}$

1.57
0.27

$\begin{array}{r}4.4 \\ 1.78 \\ \hline 1.54\end{array}$

0.24

$\begin{array}{r}4.8 \\ 0.96 \\ \hline 0.64\end{array}$

0.64

0.32

0.00

4.8
1.06

$\frac{1.06}{0.77}$

0.29

0.00

0.00

$0.00 \quad 0.00$

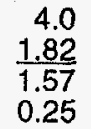

2.8
1.92
1.57
0.35

3.9

$\frac{1.82}{1.57}$

$\begin{array}{ll}1.57 & 1.57\end{array}$

$\begin{array}{ll}0.00 & 0.00\end{array}$

\begin{tabular}{r}
3.6 \\
1.83 \\
\hline 1.57 \\
0.26 \\
0.00
\end{tabular}

\begin{tabular}{r}
3.9 \\
0.93 \\
\hline 0.64 \\
0.29
\end{tabular}

3.9

$\frac{1.03}{0.77}$

0.77
0.26

$0.00 \quad 0.00$
1.5
2.31
1.42
0.89

0.89

$\frac{2.26}{1.36}$

0.90

0.00

341,637

341,637

TOTAL

118,800

$1,269,000$

$1,269,000$

135,000

135,000

417,718

417,718 


\section{Long-Term importer}

CITY OF PASADENA, CALIFORNIA (Westcoast Gas Services) AVG Daily Quantity

Total Price (Eastpont, Idaho)

Commodity Component

Demand Component

Reservation Fee Component

COLONIAL NATURAL GAS

(Alberta Northeast Gas (WGM Ltd. 1))

AVG Daily Quantity

Total Price (Waddington, NY)

Commodity Component

Demand Component

Reservation Fee Component

\section{COMMONWEALTH GAS COMPANY}

(Alberta Northeast Gas (WGM Ltd. 1))

AVG Daily Quantity

Total Price (Waddington, NY)

Commodity Component

Demand Component

Reservation Fee Component

CONNECTICUT NATURAL GAS

(Alberta NE Gas (AEC Oil \& Gas Company))

AVG Daily Quantity

Total Price (Waddington. NY)

Commodity Component

Demand Component

Reservation Fee Component

CONNECTICUT NATURAL GAS

(Alberta Northeast Gas (Atcor))

AVG Daily Quantity

Total Price (Waddington. NY)

Commodity Component

Demand Component

Reservation Fee Component
VOLUME AND PRICE REPORT

\section{Long Term Imports}

Volumes (MMct/d) \& Prices ( $\$ M M B T U$ ) of Natural

Gas Imported During the Past 12 Months 04/01/94 - 03/31/95

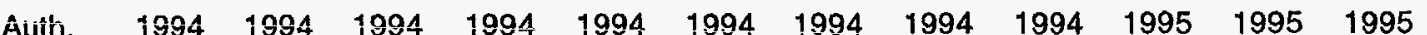

vols. Apr Mav Jun. Jul Aug. Sep. Oct Nov Dec. Jan. Feb. Mar.

4.1

$\begin{array}{rrr}4.0 & 4.1 & 4.3 \\ \frac{1.76}{1.44} & \frac{1.76}{1.44} & \frac{1.7}{1.4} \\ 0.32 & 0.32 & 0.3 \\ 0.00 & 0.00 & 0.00\end{array}$

6.0

\begin{tabular}{rr}
6.0 & 6.0 \\
2.24 & 2.1 \\
\hline 1.37 & 1.30 \\
0.87 & 0.8 \\
0.00 & 0.00
\end{tabular}

4.5

$\begin{array}{rrr}4.5 & 4.5 & \\ 2.24 & 2.17 & 2 \\ 1.37 & 1.30 & 1 \\ 0.87 & 0.87 & 0.87 \\ 0.00 & 0.00 & 0\end{array}$

1.2

\begin{tabular}{lr}
1.2 & 1.2 \\
2.17 & 2.24 \\
\hline 1.31 & 1.39 \\
0.86 & 0.85 \\
0.00 & 0.00
\end{tabular}
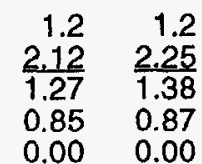

$\frac{2.23}{1.35}$

1.35
0.88

0.00

1.2
2.14
1.26
0.88

0.88
0.00

$\frac{1.96}{1.07}$

0.89
0.00

$\frac{2.36}{1.48}$

0.88

$\frac{2.42}{1.50}$

1.50
0.92
0.00

$\frac{2.38}{1.49}$

0.89
0.00

$\frac{2.31}{1.44}$

$\frac{2.31}{1.44}$
0.87

0.87
0.00

2.4

\begin{tabular}{|c|c|c|}
\hline $\begin{array}{r}2.4 \\
2.26 \\
1.39 \\
0.87 \\
0.00\end{array}$ & $\begin{array}{r}2.4 \\
2.16 \\
1.29 \\
0.87 \\
0.00\end{array}$ & $\begin{array}{r}2.4 \\
2.12 \\
1.25 \\
0.87 \\
0.00\end{array}$ \\
\hline
\end{tabular}

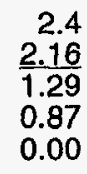

\begin{tabular}{r}
2.4 \\
2.16 \\
\hline 1.29 \\
0.87 \\
0.00
\end{tabular}

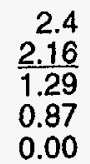

\begin{tabular}{r}
2.4 \\
2.16 \\
\hline 1.29 \\
0.87 \\
0.00
\end{tabular}
2.4
2.12
1.25

$\frac{2.12}{1.25}$

0.87

0.00

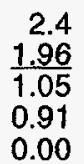

\begin{tabular}{r}
2.4 \\
1.96 \\
\hline 1.05 \\
0.91 \\
0.00
\end{tabular}

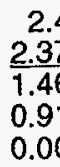

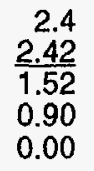

2.4
2.42
1.52
0.90
0.00

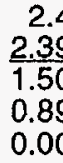

\begin{tabular}{r}
2.4 \\
2.31 \\
\hline 1.42 \\
0.89
\end{tabular}

0.89

0.00
2.26
1.36
0.90

0.90

1095

TOTAL Maf

YTD

IOTAL MAĆ́

361,614

361,614

540,000

405,000

405,000

$\frac{2.26}{1.36}$

1.36

0.00

108,540

108,540

218,160 


\section{VOLUME AND PRICE REPORT}

\section{Long Term Imports}

Volumes (MMct/d) \& Prices (\$MMBTU) of Natural

Gas Imported During the Past 12 Months 04/01/94 - 03/31/95
Leng-Term Importer

CONNECTICUT NATURAL GAS

(Alberta Northeast Gas (ProGas))

AVG Daily Quantity

Total Price (Waddington. NY)

Commodity Component

Demand Component

Reservation Fee Component

CONNECTICUT NATURAL GAS

(Alberta Northeast Gas (WGM Ltd. 1))

AVG Daily Quantity

Total Price (Waddington, NY)

Commodity Component

Demand Component

Reservation Fee Component

CONNECTICUT NATURAL GAS

(Alberta Northeast Gas (WGM Ltd. 2))

AVG Daily Quantity

Total Price (Waddington, NY)

Commodity Componen

Demand Component

Reservation Fee Component

CONSOLIDATED EDISON COMPANY OF N.Y

(Alberta NE Gas (AEC Oil \& Gas))

AVG Daily Quantity

Total Price (Waddington, NY)

Commodity Component

Demand Component

Reservation Fee Component
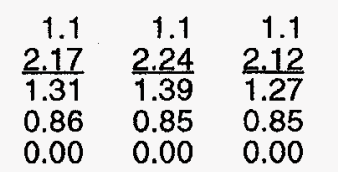

1.1
2.25
1.38
0.87
0.00

1.1
2.23
1.35
0.88
0.00

\begin{tabular}{rr}
1.1 & 1.1 \\
2.14 & 1.96 \\
\hline 1.26 & 1.07 \\
0.88 & 0.89 \\
0.00 & 0.00
\end{tabular}

1.1
2.36
1.48
0.88
0.00

1.1
2.42
1.50
0.92

$\frac{1.1}{1.38}$

1.49
0.89
0.00

$\frac{2.31}{1.44}$

1.44
0.87
0.00

CONSOLIDATED EDISON COMPANY OF N.Y. (Alberta NE Gas (Atcor))

AVG Daily Quantity

Total Price (Waddington, NY)

Commodity Component

Demand Component

Reservation Fee Component
2.6

\begin{tabular}{|c|c|c|}
\hline $\begin{array}{r}2.6 \\
2.26 \\
1.39 \\
0.87 \\
0.00\end{array}$ & $\begin{array}{r}2.6 \\
2.16 \\
1.29 \\
0.87 \\
0.00\end{array}$ & $\begin{array}{r}2.6 \\
2.12 \\
1.25 \\
0.87 \\
0.00\end{array}$ \\
\hline
\end{tabular}

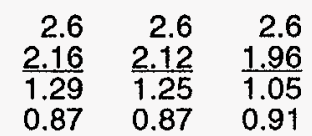

$\begin{array}{lll}1.29 & 1.25 & 1.05 \\ 0.87 & 0.87 & 0.91\end{array}$

$\begin{array}{lll}0.87 & 0.87 & 0.91 \\ 0.00 & 0.00 & 0.00\end{array}$
$2.6 \quad 2.6$

$\frac{2.37}{1.46} \quad \frac{2.42}{1.52}$

1.461 .52

$\begin{array}{ll}0.91 & 0.90 \\ 0.00 & 0.00\end{array}$

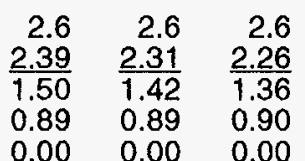

2.26

$\frac{2.26}{1.36}$

0.90
0.00

$1 \mathrm{Q95}$

TOTALMC

415,831

415,831

$\frac{2.26}{1.27}$

0.99

0.00

$1,035,000$

$1,035,000$

472,500

472,500

99,810

99,810

234,990 
VOLUME AND PRICE REPORT

\section{Long Term Imports}

Volumes (MMcf/d) \& Prices (\$MMBTU) of Natural

Gas Imported During the Past 12 Months 04/01/94 - 03/31/95
Long-Term Importer

CONSOLIDATED EDISON COMPANY OF N.Y.

(Alberta NE Gas (ProGas))

AVG Daily Quantity

Total Price (Waddington, NY)

Commodity Component

Demand Component

Reservation Fee Component

Auth

1994

Apr. 19941994

1994

\begin{tabular}{l}
1994 \\
Aug. \\
\hline
\end{tabular}

\begin{tabular}{l}
1994 \\
Sep. \\
\hline
\end{tabular}

1994

1994
ct. Nov

5.3

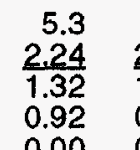

5.3
2.17
1.26
0.91
0.00

$\begin{array}{rr}5.3 & 5.2 \\ 2.12 & 2.30 \\ 1.21 & 1.35 \\ 0.91 & 0.95 \\ 0.00 & 0.00\end{array}$

5.3
2.27
1.31

$\frac{2.27}{1.31}$

5.3
2.12

5.3

0.96
0.00

1.21
0.91

0.91
0.00

$\frac{1.96}{0.99}$

0.97
0.00

$\frac{2.36}{1.39}$

1.39
0.97
0.00

$\frac{2.42}{1.44}$

0.98
0.00

$\frac{2.38}{1.38}$

CONSOLIDATED EDISON COMPANY OF N.Y.

(Alberta NE Gas (WGM Ltd. 1))

AVG Daily Quantity

5.0

$\begin{array}{rrr}5.0 & 5.0 & 5.0 \\ \frac{2.24}{1.37} & \frac{2.17}{1.30} & \frac{2.12}{1.25} \\ 0.87 & 0.87 & 0.87 \\ 0.00 & 0.00 & 0.00\end{array}$

$\frac{5.0}{1.35}$

Commodity Componen

Commodity Component

Reservation Fee Component

$0.00 \quad 0.87 \quad 0.87$

$\begin{array}{llll}0.00 & 0.00 & 0.00 & 0.00\end{array}$

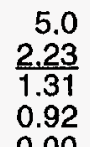

5.0
2.14
1.23
0.91
0.00

$\begin{array}{r}5.0 \\ 1.96 \\ \hline 1.04\end{array}$

CONSOUIDATED EDISON COMPANY OF N.Y.

(Alberta NE Gas (WGM Ltd. 2))

AVG Daily Quantity

Total Price (Waddington. NY)

Commodity Component

Demand Component

Reservation Fee Component

Compone

Reservation $\mathrm{Fee}$ Component

$\begin{array}{rrr}6.0 & 6.0 & 6.0 \\ 2.22 & 2.12 & 1.96 \\ 1.32 & 1.25 & 1.04 \\ 0.90 & 0.87 & 0.92 \\ 0.00 & 0.00 & 0.00\end{array}$

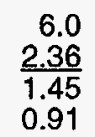

$\begin{array}{r}6.0 \\ 2.42 \\ \hline 1.52\end{array}$

6.0

0.00

$\begin{array}{ll}0.00 & 0.00\end{array}$

0.91
0.00

0.90

$\frac{2.38}{1.47}$

0.91

6.0
2.31
1.42

1.42
0.89

6.0
2.26
1.36
0.90

540,000

$31.8 \quad 31$

$\begin{array}{ll}31.8 & 31.8\end{array}$

$\frac{2.17}{1.33}$

1.33
0.84
0.00

$\frac{2.04}{1.16} \quad \frac{1.71}{0.85}$

$0.88 \quad 0.86$

31.8
1.66

$\frac{1.66}{0.79}$

31.8

0.00

0.00

CONSUMERS POWER COMPANY (1)

(WGM Ltd.)

AVG Daily Quantity

Total Price (Noyes. Minnesota)

Commodity Component

Demand Component

Reservation Fee Component

$\begin{array}{lll}15.0 & 15.0 & 15 \\ \frac{1.69}{1.26} & \frac{1.69}{1.27} & 1.71 \\ 0.43 & 0.42 & 0.45 \\ 0.00 & 0.00 & 0.00\end{array}$


VOLUME AND PRICE REPORT

\section{Long Term Imports}

Volumes (MMcf/d) \& Prices (\$MMBTU) of Natural Gas Imported During the Past 12 Months 04/01/94 - 03/31/95
Long-Term Importer

CONSUMERS POWER COMPANY 1 (1)

(Norcen Energy Resources Ltd.)

AVG Daily Quantity

Total Price (Neyes, Minnesota)

Commodity Component

Demand Component

Reservation Fee Component

CONSUMERS POWER COMPANY2 (2)

(Husky Oil)

AVG Daily Quantity

Total Price (Noyes, Minnesota)

Commodity Component

Demand Component

Reservation Fee Component

CONSUMERS POWER COMPANY3 (3)

(Shell Canada Limited)

AVG Daily Quantity

Total Price (Noyes, Minnesota)

Commodity Component

Demand Component

Reservation Fee Component

CONSUMERS POWER COMPANY4 (4)

(Poco Petroleums Ltd.)

AVG Daily Quantity

Total Price (Noyes, Minnesota)

Commodity Component

Demand Component

Reservation Fee Component

CONSUMERS POWER COMPANY5 (5)

(North Canadian Oils, Ltd.)

AVG Daily Quantity

Total Price (Noyes, Minnesota)

Commodity Component

Demand Component

Reservation Fee Component

\begin{tabular}{|c|c|c|c|c|c|c|c|c|c|c|c|c|}
\hline $\begin{array}{l}\text { Auth. } \\
\text { Vols. }\end{array}$ & $\begin{array}{l}1994 \\
\text { Apr. }\end{array}$ & $\begin{array}{l}1994 \\
\text { May. }\end{array}$ & $\begin{array}{r}1994 \\
\text { Jun. }\end{array}$ & $\begin{array}{c}1994 \\
\text { Jul. }\end{array}$ & $\begin{array}{l}1994 \\
\text { Aug. }\end{array}$ & $\begin{array}{l}1994 \\
\text { Sep. }\end{array}$ & $\begin{array}{l}1994 \\
\text { Oct. }\end{array}$ & $\begin{array}{l}1994 \\
\text { Nov. }\end{array}$ & $\begin{array}{l}1994 \\
\text { Dec. }\end{array}$ & $\begin{array}{l}1995 \\
\text { Jan. }\end{array}$ & $\begin{array}{l}1995 \\
\text { Feb. }\end{array}$ & $\begin{array}{l}1995 \\
\text { Mar. }\end{array}$ \\
\hline 14.0 & $\begin{array}{l}14.0 \\
1.77 \\
1.34 \\
0.43 \\
0.00\end{array}$ & $\begin{array}{l}14.0 \\
1.78 \\
1.35 \\
0.43 \\
0.00\end{array}$ & $\begin{array}{l}14.0 \\
2.10 \\
2.10 \\
0.00 \\
0.00\end{array}$ & $\begin{array}{l}14.0 \\
2.10 \\
2.10 \\
0.00 \\
0.00\end{array}$ & $\begin{array}{r}0.0 \\
0.00 \\
0.00 \\
0.00 \\
0.00\end{array}$ & $\begin{array}{r}0.0 \\
0.00 \\
0.00 \\
0.00 \\
0.00\end{array}$ & $\begin{array}{r}0.0 \\
0.00 \\
0.00 \\
0.00 \\
0.00\end{array}$ & $\begin{array}{r}0.0 \\
0.00 \\
0.00 \\
0.00 \\
0.00\end{array}$ & $\begin{array}{r}0.0 \\
0.00 \\
0.00 \\
0.00 \\
0.00\end{array}$ & $\begin{array}{r}0.0 \\
0.00 \\
0.00 \\
0.00 \\
0.00\end{array}$ & $\begin{array}{r}0.0 \\
0.00 \\
0.00 \\
0.00 \\
0.00\end{array}$ & $\begin{array}{r}0.0 \\
0.00 \\
0.00 \\
0.00 \\
0.00\end{array}$ \\
\hline 15.0 & $\begin{array}{l}15.0 \\
1.72 \\
1.32 \\
0.40 \\
0.00\end{array}$ & $\begin{array}{l}15.0 \\
1.73 \\
1.33 \\
0.40 \\
0.00\end{array}$ & $\begin{array}{r}0.0 \\
0.00 \\
0.00 \\
0.00 \\
0.00\end{array}$ & $\begin{array}{r}0.0 \\
0.00 \\
0.00 \\
0.00 \\
0.00\end{array}$ & $\begin{array}{r}0.0 \\
0.00 \\
0.00 \\
0.00 \\
0.00\end{array}$ & $\begin{array}{r}0.0 \\
0.00 \\
0.00 \\
0.00 \\
0.00\end{array}$ & $\begin{array}{r}0.0 \\
0.00 \\
0.00 \\
0.00 \\
0.00\end{array}$ & $\begin{array}{r}0.0 \\
0.00 \\
0.00 \\
0.00 \\
0.00\end{array}$ & $\begin{array}{r}0.0 \\
0.00 \\
0.00 \\
0.00 \\
0.00\end{array}$ & $\begin{array}{r}0.0 \\
0.00 \\
0.00 \\
0.00 \\
0.00\end{array}$ & $\begin{array}{r}0.0 \\
0.00 \\
0.00 \\
0.00 \\
0.00\end{array}$ & $\begin{array}{r}0.0 \\
0.00 \\
0.00 \\
0.00 \\
0.00\end{array}$ \\
\hline 15.0 & $\begin{array}{l}15.0 \\
1.69 \\
1.28 \\
0.41 \\
0.00\end{array}$ & $\begin{array}{l}15.0 \\
2.12 \\
2.12 \\
0.00 \\
0.00\end{array}$ & $\begin{array}{l}15.0 \\
2.12 \\
2.12 \\
0.00 \\
0.00\end{array}$ & $\begin{array}{l}11.3 \\
\frac{2.12}{2.12} \\
0.00 \\
0.00\end{array}$ & $\begin{array}{r}0.0 \\
0.00 \\
0.00 \\
0.00 \\
0.00\end{array}$ & $\begin{array}{r}0.0 \\
0.00 \\
0.00 \\
0.00 \\
0.00\end{array}$ & $\begin{array}{r}0.0 \\
0.00 \\
0.00 \\
0.00 \\
0.00\end{array}$ & $\begin{array}{r}0.0 \\
0.00 \\
0.00 \\
0.00 \\
0.00\end{array}$ & $\begin{array}{r}0.0 \\
0.00 \\
0.00 \\
0.00 \\
0.00\end{array}$ & $\begin{array}{r}0.0 \\
0.00 \\
0.00 \\
0.00 \\
0.00\end{array}$ & $\begin{array}{r}0.0 \\
0.00 \\
0.00 \\
0.00 \\
0.00\end{array}$ & $\begin{array}{r}0.0 \\
0.00 \\
0.00 \\
0.00 \\
0.00\end{array}$ \\
\hline 15.0 & $\begin{array}{l}15.0 \\
1.66 \\
1.26 \\
0.40 \\
0.00\end{array}$ & $\begin{array}{l}15.0 \\
1.65 \\
1.26 \\
0.39 \\
0.00\end{array}$ & $\begin{array}{r}0.0 \\
0.00 \\
0.00 \\
0.00 \\
0.00\end{array}$ & $\begin{array}{r}0.0 \\
0.00 \\
0.00 \\
0.00 \\
0.00\end{array}$ & $\begin{array}{r}0.0 \\
0.00 \\
0.00 \\
0.00 \\
0.00\end{array}$ & $\begin{array}{r}0.0 \\
0.00 \\
0.00 \\
0.00 \\
0.00\end{array}$ & $\begin{array}{r}0.0 \\
0.00 \\
0.00 \\
0.00 \\
0.00\end{array}$ & $\begin{array}{r}0.0 \\
0.00 \\
0.00 \\
0.00 \\
0.00\end{array}$ & $\begin{array}{r}0.0 \\
0.00 \\
0.00 \\
0.00 \\
0.00\end{array}$ & $\begin{array}{r}0.0 \\
0.00 \\
0.00 \\
0.00 \\
0.00\end{array}$ & $\begin{array}{r}0.0 \\
0.00 \\
0.00 \\
0.00 \\
0.00\end{array}$ & $\begin{array}{r}0.0 \\
0.00 \\
0.00 \\
0.00 \\
0.00\end{array}$ \\
\hline 10.0 & $\begin{array}{l}10.0 \\
1.69 \\
1.29 \\
0.40 \\
0.00\end{array}$ & $\begin{array}{l}10.0 \\
1.66 \\
1.27 \\
0.39 \\
0.00\end{array}$ & $\begin{array}{r}0.0 \\
0.00 \\
0.00 \\
0.00 \\
0.00\end{array}$ & $\begin{array}{r}0.0 \\
0.00 \\
0.00 \\
0.00 \\
0.00\end{array}$ & $\begin{array}{r}0.0 \\
0.00 \\
0.00 \\
0.00 \\
0.00\end{array}$ & $\begin{array}{r}0.0 \\
0.00 \\
0.00 \\
0.00 \\
0.00\end{array}$ & $\begin{array}{r}0.0 \\
0.00 \\
0.00 \\
0.00 \\
0.00\end{array}$ & $\begin{array}{r}0.0 \\
0.00 \\
0.00 \\
0.00 \\
0.00\end{array}$ & $\begin{array}{r}0.0 \\
0.00 \\
0.00 \\
0.00 \\
0.00\end{array}$ & $\begin{array}{r}0.0 \\
0.00 \\
0.00 \\
0.00 \\
0.00\end{array}$ & $\begin{array}{r}0.0 \\
0.00 \\
0.00 \\
0.00 \\
0.00\end{array}$ & $\begin{array}{r}0.0 \\
0.00 \\
0.00 \\
0.00 \\
0.00\end{array}$ \\
\hline
\end{tabular}

1095

TOTAL MCf

TOTALMCf

0

0

0

1) New short-term contract replaced this contract; beginning in August, Consumers Power began using its short-term import authorization for these volumes.

New short-term contract replaced this contract; beginning in June. Husky and Consumers Power began using their short-term import authorizations for these volumes.

New sher these volumes.

(5) New short-term contract replaced this contract; beginning in August, Consumers Power began using its short-lerm import authorization for these volumes. 
VOLUME AND PRICE REPORT

Long Term Imports

Volumes (MMcf/d) \& Prices (\$MMBTU) of Natural

Gas Imported During the Past 12 Months 04/01/94 - 03/31/95
Long-Term Importer

CRESTAR ENERGY MARKETING CORPORATION (1) (Crestar Energy)

AVG Daily Quantity

Total Price (Noyes. Minnesota)

Commodity Component

Demand Component

Reservation Fee Component

Auth.
Vols.
N (1)
15.0

$1994 \quad 1994 \quad 1994 \quad 1994 \quad 1994$

Apr. May

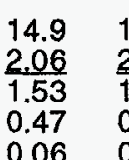

\begin{tabular}{ll}
14.9 & 15.4 \\
2.04 & 1.79 \\
\hline 1.53 & 1.28 \\
0.45 & 0.46 \\
0.06 & 0.05
\end{tabular}

14.9
1.92
1.40
0.46
0.06

14.8
1.84

1.33

0.06

3.0

$\begin{array}{rrr}1.3 & 1.6 & 1 \\ \frac{3.07}{1.64} & \frac{2.65}{1.64} & \frac{2.5}{1.64} \\ 1.43 & 1.01 & 0.93 \\ 0.00 & 0.00 & 0.00\end{array}$

5.0

$\begin{array}{rrr}1.9 & 2.7 & 3.4 \\ \frac{3.07}{1.64} & 2.65 & 2.57 \\ 1.43 & 1.64 & 1.64 \\ 0.00 & 0.00 & 0.03\end{array}$

2.0

$\begin{array}{rrr}0.9 & 1.3 & 1.3 \\ \frac{3.07}{1.64} & 2.65 & \frac{2.57}{1.64} \\ 1.64 \\ 0.43 & 1.01 & 0.93 \\ 0.00 & 0.00 & 0.00\end{array}$
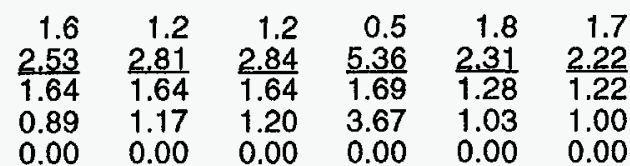

1.8
2.21
1.30
0.91
0.00

Reservation Fee Component

DARTMOUTH POWER ASSOCIATES, L.P. (2)

Home Oil Company Limited)

AVG Daily Quantity

\begin{tabular}{rrr}
0.0 & 0.0 & 0.0 \\
0.00 & $\underline{0.00}$ & $\underline{0.00}$ \\
\hline 0.00 & 0.00 & 0.00 \\
0.00 & 0.00 & 0.00 \\
0.00 & 0.00 & 0.00
\end{tabular}

\begin{tabular}{r}
0.0 \\
0.00 \\
\hline 0.00 \\
0.00 \\
0.00
\end{tabular}

\begin{tabular}{r}
0.0 \\
0.00 \\
\hline 0.00 \\
0.00 \\
0.00
\end{tabular}

0.00

\begin{tabular}{r}
0.0 \\
0.00 \\
\hline 0.00 \\
0.00
\end{tabular}

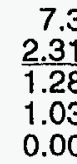

0.0
0.00
0.00
0.00
0.00
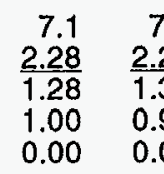

$\frac{7.21}{1.30}$ 0.91 0.00 $\frac{1.7}{1.23}$

14.9

$\frac{1.57}{1.02}$

14.9

$\frac{1.54}{1.01}$

0.46

0.07

0.06

(1) Supply is sold to Northern States Power Company for retail customers in the Grand Forks and Fargo areas of North Dakola.
(2) Contract with Home Oil Limited commenced 11/01/94. Contracts with Anderson Oil \& Gas and Canadian Nalural Gas Resources were terminaled effective 10/31/94. 
VOLUME AND PRICE REPORT

\section{Long Term Imports}

Volumes (MMcf/d) \& Prices (\$MMBTU) of Natural Gas Imported During the Past 12 Months 04/01/94 - 03/31/95

Long-Term Importer

DARTMOUTH POWER ASSOCIATES, L.P.

(Remington Energy Ltd.)

AVG Daily Quantity

Total Price (Waddington. NY)

Commodity Component

Demand Component

Reservation Fee Component

DISTRIGAS CORPORATION (88-37-LNG)

(Sonatrach)

Total Price (Everett. Massachusetts)

Commodity Component

Demand Component

Reservation Fee Component

DISTRIGAS CORPORATION (89-16-LNG)

(Sonatrach)

AVG Daily Quantity

Total Price (Everett, Massachusetts)

Commodity Component

Demand Component

Reservation Fee Component

ELIZABETHTOWN GAS COMPANY

(WGM Ltd.)

AVG Daily Quantity

Total Price (Niagara Falls. NY)

Commodity Component

Demand Component

Reservation Fee Component

ENCOGEN FOUR PARTNERS, L.P. (1)

(Sceptre Resources Limited)

AVG Daily Quantity

Total Price (Niagara Falls, NY)

Commodity Component

Demand Component

Reservation Fee Component
AVG Daily Quantity $\begin{array}{lllllllllllll}\text { Auth. } & 1994 & 1994 & 1994 & 1994 & 1994 & 1994 & 1994 & 1994 & 1994 & 1995 & 1995 & 1995\end{array}$

Vols. Apr. May. Jun. Jul. Aug. Sep. Oct. Nov Dec. Jan. Feb. Mar.

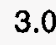

$\begin{array}{rrrr}2.6 & 3.6 & 3.7 & 5.0 \\ \frac{3.07}{1.64} & 2.65 & 2.57 & 2.53 \\ 1.43 & 1.04 & 1.64 & 1.64 \\ 0.00 & 0.00 & 0.93 & 0.89 \\ & & 0.00 & 0.00\end{array}$

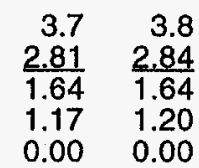

$\begin{array}{r}1.3 \\ 5.36 \\ \hline 1.69\end{array}$

$\frac{5.36}{1.69}$

0.0

$\begin{array}{lll}82.2 & 83.9 & 0.0 \\ \frac{2.17}{2.17} & \frac{2.18}{2.18} & \underline{0.00} \\ 0.00 & 0.00 & 0.00\end{array}$

$\begin{array}{lll}0.00 & 0.00 & 0.00\end{array}$

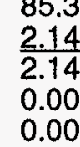

$80.4 \quad 83.4$

$\frac{2.19}{2.19} \quad \frac{2.15}{2.15}$

$0.19 \quad 2.15$

$\begin{array}{ll}0.00 & 0.00 \\ 0.00 & 0.00\end{array}$

0.0

\begin{tabular}{r}
0.0 \\
0.00 \\
\hline 0.00 \\
0.00
\end{tabular}

80.7
2.11
2.11
0.00
0.00

83.0

$\frac{2.17}{2.17}$

0.00

0.00

0.00

$0.00 \quad 0.00$

10.0

$\begin{array}{ll}10.0 & 10.0 \\ 2.51 & 2.62 \\ 1.46 & 1.62 \\ 1.05 & 1.00 \\ 0.00 & 0.00\end{array}$

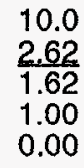

\begin{tabular}{r}
9.9 \\
2.78 \\
\hline 1.73 \\
1.05 \\
0.00
\end{tabular}

10.0
2.90
1.88
1.02
0.00

$\begin{array}{ll}10.0 & 10.0 \\ 2.78 & 2.63 \\ 1.76 & 1.58 \\ 1.02 & 1.05 \\ 0.00 & 0.00\end{array}$

10.0
2.53
1.49
1.04
0.00

10.0
2.64
1.59
1.05
0.00

10.1
2.75
1.75
1.00

\begin{tabular}{l}
10.2 \\
2.77 \\
\hline
\end{tabular}

$\frac{2.77}{1.69}$

1.08

\begin{tabular}{l}
10.2 \\
2.52 \\
\hline
\end{tabular}

1.54
0.98

0.00

15.6

$\begin{array}{llllllllllll}13.3 & 13.6 & 13.8 & 13.3 & 10.1 & 12.5 & 13.8 & 13.9 & 14.4 & 14.6 & 14.7 & 14.1 \\ \frac{2.95}{2.01} & \frac{2.89}{2.02} & \frac{2.99}{2.07} & \frac{3.09}{2.17} & \frac{3.21}{2.08} & \frac{3.02}{2.02} & \frac{2.85}{1.95} & \frac{2.92}{2.00} & \frac{2.82}{1.99} & \frac{2.90}{2.09} & \frac{2.98}{2.08} & \frac{2.93}{2.08} \\ 0.94 & 0.87 & 0.92 & 0.92 & 1.13 & 1.00 & 0.90 & 0.92 & 0.83 & 0.81 & 0.90 & 0.85 \\ 0.00 & 0.00 & 0.00 & 0.00 & 0.00 & 0.00 & 0.00 & 0.00 & 0.00 & 0.00 & 0.00 & 0.00\end{array}$

0.91

0.00
10.0
2.55
1.54
1.01
0.00

1095 TOTAL MC

246,309

TOTAL MC

246,309

$5,131,666$

$5,131,666$

(2)

0

0

912,260

912,260

(1) Supply used to fuel a 62 Megawatl gas-fired cogeneration facility located at Buffalo, New York. 


\section{VOLUME AND PRICE REPORT}

\section{Long Term Imports}

Volumes (MMcf/d) \& Prices (\$MMBTU) of Natural

Gas Imported During the Past 12 Months 04/01/94 - 03/31/95

Long-Term Importer

ENCOGEN NORTHWEST, L.P. (1)

(CanWest Gas Supply, Inc.)

AVG Daily Quantity

Total Price (Sumas, Washington)

Commodity Component

Demand Component

Reservation Fee Component

(Alberta Northeast Gas (WGM Ltd. 1))

AVG Daily Quantity

Total Price (Waddington. NY)

Demand Component

Reservation Fee Component

ESSEX COUNTY GAS CO.

(Alberta Northeast Gas (WGM Ltd. 1))

AVG Daily Quantity

Commodity Component

Demand Component

Reservation Fee Component

FULTON COGENERATION ASSOCIATES

Total Price (Niagara Falls, NY)

Commodity Component

Demand Component

Reservation Fee Component

GRANITE STATE GAS TRANSMISSION, INC

(Direct Energy Marketing Ltd.)

AVG Daily Quantity

Total Price (North Troy, VT)

Commodity Componen

Reservation Fee Component
Auth. $\quad 1994 \quad 1994 \quad 1994 \quad 1994$

Vols.

Apr.

9.6

$\begin{array}{rrr}8.2 & 7.7 & \\ \frac{1.84}{1.84} & \frac{1.84}{1.84} & \frac{1.84}{1.84} \\ 0.00 & 0.00 & 0.0 \\ 0.00 & 0.00 & 0.00\end{array}$

4.0

\begin{tabular}{rr}
4.0 & 4 \\
2.24 & 2.17 \\
\hline 1.37 & 1.30 \\
0.87 & 0.87 \\
0.00 & 0.00
\end{tabular}

2.0

$\begin{array}{rr}2.0 & 2.0 \\ \frac{2.24}{1.37} & 2.17 \\ 0.87 & 0.3 \\ 0.00 & 0.0\end{array}$

6.0

\begin{tabular}{rr}
5.9 & 6.0 \\
2.60 & 2.5 \\
\hline 1.77 & 1.7 \\
0.83 & 0.8 \\
0.00 & 0.00
\end{tabular}

6.0

$\begin{array}{rr}6.0 & 6.0 \\ 2.31 & 2.28 \\ 1.28 & 1.28 \\ 1.03 & 1.00 \\ 0.00 & 0.00\end{array}$

\begin{tabular}{rr}
9.3 & 8.4 \\
1.84 & 1.93 \\
\hline 1.84 & 1.93 \\
0.00 & 0.00 \\
0.00 & 0.00
\end{tabular}

$\begin{array}{rrr}4.0 & 4.0 & 4.0 \\ 2.17 & 2.12 & 2.25 \\ 1.30 & \frac{1.25}{1.35} \\ 0.87 & 0.87 & 0.90\end{array}$

8.7
1.93
1.93

0.00
0.00

$\frac{1.93}{1.93}$

0.00

\begin{tabular}{r}
8.2 \\
1.93 \\
\hline 1.93 \\
0.00 \\
0.00
\end{tabular}

\begin{tabular}{r}
8.2 \\
1.93 \\
\hline 1.93 \\
0.00 \\
0.00
\end{tabular}

$\frac{2.23}{1.31}$

4.0
2.14
1.23
0.91

4.0
1.96
1.04

1.04
0.92
0.00

3.9
2.36
1.45
0.91
0.00
ENERGYNORTH, INC.

Commodity Component

Total Price (Waddington, NY)

(Star Oil \& Gas Limited)

AVG Daily Quantity

Demand Component

8.2
$\frac{1.93}{1.93}$
0.00
0.00

$\frac{9.3}{1.93}$

$\frac{1.93}{1.93}$

$\begin{array}{ll}1.93 & 1.93 \\ 0.00 & 0.00\end{array}$

3.7

1.93

0.00

4.0
2.6
36
90

360,000

360,000

4.0
2.42
1.52

1.52
0.90

$\begin{array}{r}4.0 \\ 2.38 \\ \hline 1.47\end{array}$

0.91

$\frac{2.31}{1.42}$

$\begin{array}{ll}0.89 & 0.90\end{array}$

0.00

2.0

$\frac{2.26}{1.36}$

1.36

0.90

0.00

(1) Supply used to fuel a 160 Megawatt gas-fired cogeneration facility located at Bellingham, Washington. 


\section{VOLUME AND PRICE REPORT}

\section{Ong Term Imports}

Volumes (MMct/d) \& Prices (\$/MMBTU) of Natural

Gas Imported During the Past 12 Months 04/01/94 - 03/31/95

Long-Term Importer

GRANITE STATE GAS TRANSMISSION, INC.

(Shell Canada Limited)

AVG Daily Quantity

Total Price (North Troy. VT)

Commodity Component

Demand Component

Reservation Fee Component

GRANITE STATE GAS TRANSMISSION, INC.

(Shell Canada Limited)

AVG Daily Quantity

Total Price (Waddington, NY)

Commodity Componen

Demand Component

Reservation Fee Component

GREAT FALLS GAS COMPANY

(Shell Canada Limited)

AVG Daily Quantity

Total Price (Babb. Montana)

Commodity Component

Demand Component

Reservation Fee Component

Auth.

Vols.

Apr. May. Jun

1994

$\begin{array}{lll}1994 & 1994 \quad 1994\end{array}$

$1994 \quad 1994 \quad 1995$

$$
\begin{aligned}
& 24.0 \\
& 2.36 \\
& 1.33 \\
& 1.03 \\
& 0.00
\end{aligned}
$$

25.1
2.39
1.36
1.03
0.00

25.1
2.43
1.40
1.03
0.00

24.4
2.51
1.49
1.02
0.00
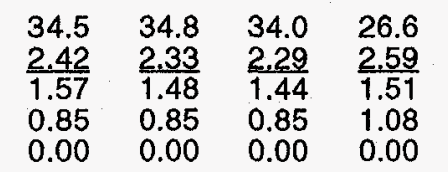

$\frac{2.59}{1.51}$

$0.00 \quad 0.00$

5.0

\begin{tabular}{|c|c|c|}
\hline $\begin{array}{r}5.0 \\
1.88 \\
1.88 \\
0.00 \\
0.00\end{array}$ & $\begin{array}{r}5.0 \\
1.62 \\
1.62 \\
0.00 \\
0.00\end{array}$ & $\begin{array}{r}5.0 \\
1.54 \\
1.54 \\
0.00 \\
0.00\end{array}$ \\
\hline
\end{tabular}

10.0

$\begin{array}{rrr}9.9 & 9.9 & 9.9 \\ \frac{1.95}{1.10} & \frac{1.71}{1.08} & \frac{1.5}{0.87} \\ 0.65 & 0.52 & 0.5 \\ 0.20 & 0.11 & 0.13\end{array}$

(Indeck Gas Supply Corp.)

AVG Daily Quantity

Total Price (Niagara Falls, NY)

Commodity Component

Demand Component

Reservation Fee Component

$\begin{array}{rrrrrrrrrrrr}9.5 & 8.3 & 9.7 & 9.6 & 9.6 & 9.6 & 9.6 & 9.5 & 8.2 & 9.6 & 9.6 & 9.7 \\ 2.54 & 2.54 & 2.54 & 2.56 & 2.56 & 2.56 & 2.54 & 2.55 & 2.54 & 2.61 & 2.61 & 2.62 \\ 1.86 & 1.86 & 1.86 & 1.86 & 1.85 & 1.85 & 1.86 & 1.86 & 1.86 & 1.92 & 1.92 & 1.92 \\ 0.68 & 0.68 & 0.68 & 0.70 & 0.71 & 0.71 & 0.68 & 0.69 & 0.68 & 0.69 & 0.69 & 0.70 \\ 0.00 & 0.00 & 0.00 & 0.00 & 0.00 & 0.00 & 0.00 & 0.00 & 0.00 & 0.00 & 0.00 & 0.00\end{array}$

24.6
2.45
1.41
1.04
0.00

YTD

TOTAL Mc

$2,202,823$

$\frac{2.46}{1.21} \quad \frac{2.54}{1.26}$

$\begin{array}{ll}1.25 & 1.28\end{array}$

21.1
2.64

1.36
1.28

0.00

$23.4 \quad 23.9$

$\frac{2.51}{1.42} \quad \frac{2.41}{1.36}$

$1.09 \quad 1.05$

24.7

$\frac{2.46}{1.31}$

24.8
2.32

1.29
1.03

0.00

$2,202,823$

$3,077,015$

$3,077,015$

$\begin{array}{lll}2.40 & 2.43 & 27.6 \\ 1.49 & \frac{2.25}{1.38} & \frac{1.18}{1.18}\end{array}$

0.91

0.91
0.00

0.00

$\frac{2.26}{1.36}$

\begin{tabular}{l}
34.8 \\
2.26 \\
\hline
\end{tabular}

1.43

33.2

$\frac{2.25}{1.40} \quad \frac{2.19}{1.26}$

34.7
2.14

$\frac{2.14}{1.31}$

0.83

0.00

450,049

450,049

$\begin{array}{rrr}5.0 & 5.0 & 2.0 \\ 1.40 & \frac{1.41}{1.41} & \frac{1.53}{1.53} \\ 0.00 & 0.00 & 0.00 \\ 0.00 & 0.00 & 0.00\end{array}$

$\begin{array}{r}5.0 \\ 1.62 \\ \hline 1.62\end{array}$

1.62
0.00

$\begin{array}{r}5.0 \\ 1.60 \\ \hline 1.60\end{array}$

$\frac{1.26}{1.26}$

\begin{tabular}{r}
5.0 \\
1.47 \\
\hline 1.47 \\
0.00
\end{tabular}

$\frac{1.28}{1.28}$

1.28
0.00

0.00

0.00

$\frac{0.00}{0.00}$

0.00

866,563 


\section{VOLUME AND PRICE REPORT}

\section{Long Term Imports}

Volumes (MMct/d) \& Prices (\$MMBTU) of Natural

Gas Imported During the Past 12 Months 04/01/94 - 03/31/95

Long-Term Importer

(Indeck Gas Supply Corp.)

AVG Daily Quantity

Total Price (Niagara Falls, NY)

Commodity Component

Demand Component

Reservation Fee Component

INDECK-YERKES LIMITED PARTNERSHIP (1)

(Northstar Energy Corporation)

AVG Daily Quantity

Total Price (Niagara Falls, NY)

Commodity Component

Demand Component

Resenvation Fee Component

KAMINE/BESICORP CARTHAGE L.P

(Renaissance Energy Ltd.)

AVG Daily Quantity

Total Price (Grand Island. NY)

Commodity Component

Demand Component

Reservation Fee Component

KAMINE/BESICORP NATURAL DAM L.P.

(North Canadian Marketing Inc.)

AVG Daily Quantity

Total Price (Waddington, NY)

Demand Component

Reservation Fee Component

KAMINE/BESICORP SOUTH GLENS FALLS L.P.

AVG Daily Quantity

Total Price (Noyes, Minnesota)

Commodity Component

Demand Component

Reservation Fee Component
INDECK-YERKES ENERGY SERVICES, INC.

Commodity Component

(Renaissance Energy Ltd.)

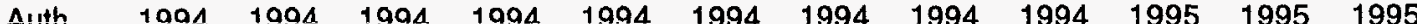

Vols. Apr May Jun Jul Aug. Sep. Oct Nov. Dec. Jan Fob. Mar.

$\begin{array}{rr}8.3 & 9.3 \\ \frac{2.30}{1.63} & 2.2 \\ 0.67 & 0.6 \\ 0.00 & 0.00\end{array}$
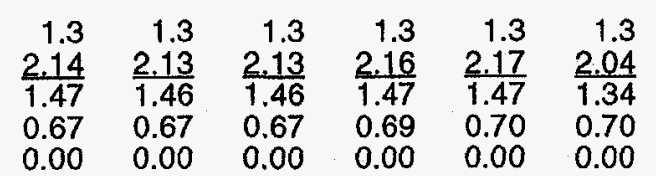

1.3
2.00
1.31
0.69

0.69

0.00
0.00
0.00

$\frac{0.00}{0.00}$

0.00

0.00
0.00

14.2

$\begin{array}{lll}12.4 & 12.3 & 11.6 \\ \frac{2.56}{1.88} & \frac{2.49}{1.84} & 2.54 \\ 0.68 & 0.65 & 0.69 \\ 0.00 & 0.00 & 0.00\end{array}$

$\begin{array}{ll}0.00 & 0.00\end{array}$

0.00

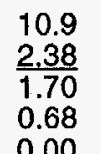

9.3
2.52
1.70

13.0
2.39
1.68
0.71

\begin{tabular}{l}
13.3 \\
2.31 \\
\hline 1.63 \\
0.68
\end{tabular}

7.1
2.61
1.56
1.05
0.00

8.5
2.34
1.59
0.75

0.82
0.00

0.00

12.5

$\begin{array}{lll}12.0 & 11.6 & 10 . \\ 2.44 & \frac{2.40}{1.73} & \frac{2.53}{1.73} \\ 1.75 & 1.67 & 0.80 \\ 0.69 & 0.67 & 0.00\end{array}$

14.2

\begin{tabular}{rrr}
12.7 & 12.4 & 9.5 \\
2.02 & $\frac{1.99}{1.75}$ & $\frac{2.00}{1.75}$ \\
\hline 1.77 & 1.75 & 0.25 \\
0.25 & 0.24 & 0.00 \\
0.00 & 0.00 & 0.00
\end{tabular}

12.4
1.87
1.62
0.25
0.00

\begin{tabular}{l}
12.2 \\
1.87 \\
\hline 1.62 \\
0.25 \\
0.00
\end{tabular}

\begin{tabular}{l}
12.6 \\
1.88 \\
\hline 1.62 \\
0.26 \\
0.00
\end{tabular}

\begin{tabular}{l}
12.8 \\
1.80 \\
\hline 1.55 \\
0.25 \\
0.00
\end{tabular}

\begin{tabular}{r}
7.0 \\
1.74 \\
\hline 1.49 \\
0.25 \\
0.00
\end{tabular}

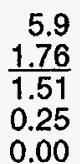

5.9
1.76
1.51
0.25
0.00

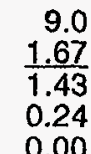

\begin{tabular}{r}
9.0 \\
1.67 \\
\hline 1.43 \\
0.24 \\
0.00
\end{tabular}

$\frac{2.32}{1.63}$

1.63

0.69

0.00

$\frac{2.07}{1.27}$

1.27

0.00
1Q95

TOTAL Mct

YTD

TOTAL MCA

811,128

811,128

0

0

827,166

827,166

(1) Contract with Northstar was terminated on 11/01/94 


\section{VOLUME AND PRICE REPORT}

\section{Long Term Imports}

Volumes (MMcf/d) \& Prices (\$MMBTU) of Natural Gas Imported During the Past 12 Months 04/01/94 - 03/31/95

Vols

Long-Term Importer

KAMINE/BESICORP SYRACUSE L.P.

(North Canadian Marketing Inc.)

AVG Daily Quantity

Total Price (Grand Is and NY)

Commodity Component

Demand Component

Reservation Fee Component

KCS ENERGY MARKETING, INC. (1)

(Ramarro Resources Ltd.

AVG Daily Quantity

Total Price (Niagara Falls, NY)

Commodity Component

Demand Component

Reservation Fee Component

LG\&E WESTMORELAND RENSSELAER (WGM Ltd.)

AVG Daily Quantity

Total Price (Niagara Falls. NY)

Commodity Component

Demand Component

Reservation Fee Component

LOCKPORT ENERGY ASSOCIATES, L.P.

(ProGas Limited)

AVG Daily Quantity

Total Price (Niagara Falls, NY)

Commodity Component

Demand Component

Reservation Fee Component

LONG ISLAND LIGHTING COMPANY

(Alberta NE Gas (AEC Oil \& Gas))

AVG Daily Quantity

Total Price (Waddington, NY)

Commodity Component

Demand Component

Reservation Fee Component
16.3

$\begin{array}{lll}12.3 & 11.8 & 1 \\ 2.45 & 2.52 & 2.39 \\ 1.78 & 1.77 & 1.77 \\ 0.67 & 0.75 & 0.72 \\ 0.00 & 0.00 & 0.00\end{array}$

6.0

$\begin{array}{rrr}6.1 & 6.0 & 6 \\ \frac{2.39}{1.72} & \frac{2.44}{1.79} & \frac{2.3}{1.6} \\ 0.67 & 0.65 & 0.68 \\ 0.00 & 0.00 & 0.00\end{array}$

18.0

$\begin{array}{lll}15.8 & 18.3 & 18 \\ \frac{2.61}{1.40} & \frac{2.42}{1.39} & \frac{2.3}{1.2} \\ 1.21 & 1.03 & 1.07 \\ 0.00 & 0.00 & 0.00\end{array}$

12.0

$\begin{array}{rrr}10.1 & 9.7 & 7.7 \\ \frac{3.24}{2.17} & \frac{3.25}{2.15} & \frac{3.62}{2.14} \\ 1.03 & 1.06 & 1.42 \\ 0.04 & 0.04 & 0.06\end{array}$

3.1

$\begin{array}{rrrrrr}3.1 & 3.1 & 3.1 & 3.1 & 3.1 & 3.1 \\ \frac{2.17}{1.31} & \frac{2.24}{1.39} & \frac{2.12}{1.27} & \frac{2.25}{1.38} & \frac{2.23}{1.35} & \frac{2.14}{1.26} \\ 0.86 & 0.85 & 0.85 & 0.87 & 0.88 & 0.88 \\ 0.00 & 0.00 & 0.00 & 0.00 & 0.00 & 0.00\end{array}$

Not. Nov.
Dec.
1995

Jan.

$\begin{array}{lll}.3 & 13.5 & 11.6 \\ .9 & 2.44 & 2.65 \\ .77 & 1.77 & 1.77 \\ .72 & 0.67 & 0.88 \\ 00 & 0.00 & 0.00\end{array}$

15.0
2.52
1.76
0.76
0.00

14.6
2.44
1.77
0.67
0.00

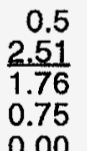

\begin{tabular}{r}
0.5 \\
2.48 \\
\hline 1.77 \\
0.71 \\
0.00
\end{tabular}

\begin{tabular}{r}
0.5 \\
2.37 \\
\hline 1.68 \\
0.69 \\
0.00
\end{tabular}

\begin{tabular}{rr}
0.8 & 0.5 \\
2.57 & 2.42 \\
\hline 1.84 & 1.71 \\
0.73 & 0.71 \\
0.00 & 0.00
\end{tabular}

$0.00 \quad 0.00$

6.0

$\frac{1.72}{1.03}$

0.69

18.2

$\frac{2.17}{1.17}$

1.17

1.00

0.00

$\begin{array}{llll}. .40 & 1.35 & 1.22 & 1.13 \\ 1.05 & 1.14 & 1.12 & 1.04\end{array}$

1.04
0.00

$\frac{2.61}{1.27}$

1.34
0.00

0.00

0.00

(1) Gas used to fuel a cogeneration facility in Milford, New Jersey (formerly Energy Marketing Exchange, Inc.) 
Long-Term Importer

LONG ISLAND LIGHTING COMPANY (Alberta Northeast Gas (Atcor))

AVG Daily Quantity

Total Price (Waddington. NY)

Commodity Component

Demand Component

Reservation Fee Component

LONG ISLAND LIGHTING COMPANY

(Alberta Northeast Gas (ProGas))

AVG Daily Quantity

Total Price (Waddington, NY)

Commodity Component

Demand Component

Reservation Fee Component

LONG ISLAND LIGHTING COMPANY (Alberta Northeast Gas (WGM Ltd. 1))

AVG Daily Quantity

Total Price (Waddington, NY)

Commodity Component

Demand Component

Reservation Fee Component

LONG ISLAND LIGHTING COMPANY (Alberta Northeast Gas (WGM Ltd. 2))

AVG Daily Quantity

Total Price (Waddington. NY)

Commodity Component

Demand Component

Reservation Fee Component

MEGAN-RACINE ASSOCIATES, INC. (WGM Ltd.)

AVG Daily Quantity

Total Price (Massena. New York)

Commodity Component

Demand Component

Reservation Fee Component

\section{VOLUME AND PRICE REPORT}

\section{Long Term Imports}

Volumes (MMct/d) \& Prices (\$MMBTU) of Natural

Gas Imported During the Past 12 Months 04/01/94 - 03/31/95

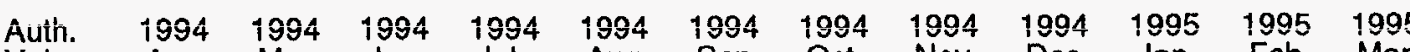

Vols. Apr. May. Jun. Jul. Aug. Sep. Oct. Nov. Dec. Jan. Feb. Mar.

6.2

\begin{tabular}{rrr}
6.2 & 6.2 & 6.2 \\
2.26 & 2.16 & 2.12 \\
\hline 1.39 & 1.29 & 1.25 \\
0.87 & 0.87 & 0.87 \\
0.00 & 0.00 & 0.00
\end{tabular}

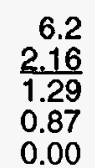

6.2
2.16

$\frac{2.16}{1.29}$

0.00

6.2
2.12
1.25
0.87
0.00

\begin{tabular}{r}
6.2 \\
1.96 \\
\hline 1.05 \\
0.91 \\
0.00
\end{tabular}

6.2
2.37
1.46
0.91
0.00

6.2
2.42
1.52
0.90

$\begin{array}{r}6.2 \\ 2.39 \\ \hline 1.50\end{array}$

0.89

$\begin{array}{r}6.2 \\ 2.31 \\ \hline 1.42\end{array}$

1.42
0.89

11.9

$\begin{array}{ll}11.9 & 11.9 \\ 2.24 & 2.1 \\ 1.32 & 1.26 \\ 0.92 & 0.91 \\ 0.00 & 0.00\end{array}$

\begin{tabular}{l}
11.9 \\
2.17 \\
\hline 1.26 \\
0.91 \\
0.00
\end{tabular}

11.9
2.12

$\frac{2.12}{1.21}$

1.21
0.91

11.8
2.30
1.35
0.95

11.9
2.27
1.31
0.96

11.9
2.1
1.21
0.91

\begin{tabular}{l}
11.9 \\
1.96 \\
\hline
\end{tabular}

0.00

30.4

$\begin{array}{ll}30.4 & 30 . \\ 2.24 & 2.17 \\ 1.37 & 1.34 \\ 0.87 & 0.87 \\ 0.00 & 0.06\end{array}$

\begin{tabular}{ll}
30.4 & 30.4 \\
2.17 & 2.1 \\
\hline 1.30 & 1.25 \\
0.87 & 0.87 \\
0.00 & 0.0
\end{tabular}

$\begin{array}{ll}30.4 & 3 \\ 2.12 & 2 \\ 1.25 & 1 \\ 0.87 & 0 \\ 0.00 & 0\end{array}$

\begin{tabular}{ll}
30.4 & 30.4 \\
2.25 & 2.23 \\
\hline 1.35 & 1.3 \\
0.90 & 0.9 \\
0.00 & 0.00
\end{tabular}

$30.4 \quad 30.4$

$\frac{2.14}{1.23}$

$\begin{array}{ll}2 & 0.91 \\ 0 & 0.00\end{array}$

30.4
1.96

$\frac{1.96}{1.04}$

0.92

$\frac{2.36}{1.45}$

0.91
0.00

\begin{tabular}{l}
30.4 \\
2.42 \\
\hline
\end{tabular}

$\frac{2.42}{1.52}$

1.52
0.90

0.00

30.4
$\frac{2.38}{1.47}$

1.47
0.91

0.91
0.00

30.0
2.31
1.42

1.42

0.89
0.00

$\begin{array}{ll}13.5 & 13.5 \\ 2.24 & 2 \\ 1.37 & 1 . \\ 0.87 & 0.87 \\ 0.00 & 0.00\end{array}$

\begin{tabular}{ll}
13.5 & 13.5 \\
2.17 & 2.12 \\
\hline 1.30 & 1.25 \\
0.87 & 0.87 \\
0.00 & 0.00
\end{tabular}

13.5
2.17
1.30
0.87
0.00

13.5
2.22
1.32
0.90
0.00

\begin{tabular}{l}
13.5 \\
2.12 \\
\hline 1.25 \\
0.87 \\
0.00
\end{tabular}

13.5
1.96
1.04
0.92
0.00

13.5
2.36
1.45
0.91
0.00

13.5
$\frac{2.42}{1.52}$
0.90
0.00

$\begin{array}{r}0.0 \\ 0.00 \\ \hline 0.00\end{array}$

\begin{tabular}{l}
14.9 \\
2.38 \\
\hline 1.47
\end{tabular}

$\begin{array}{lll}0.00 & 1.47 & 1.36 \\ 0.00 & 0.91 & 0.90\end{array}$

$\begin{array}{lll}0.00 & 0.00 & 0.00\end{array}$

11.7

$\begin{array}{rrrrr}10.2 & 5.3 & 8.1 & 9.4 & 9.2 \\ \frac{2.81}{1.57} & \frac{3.87}{1.57} & \frac{3.12}{1.57} & \frac{2.81}{1.54} & \frac{2.8}{1.5} \\ 1.24 & 2.30 & 1.55 & 1.27 & 1.30 \\ 0.00 & 0.00 & 0.00 & 0.00 & 0.00\end{array}$

9.6
2.82
1.54
1.28
0.00

\begin{tabular}{r}
7.3 \\
3.06 \\
\hline 1.42 \\
1.64 \\
0.00
\end{tabular} \begin{tabular}{r}
7.4 \\
3.09 \\
\hline 1.42 \\
1.67 \\
0.00
\end{tabular}

10.8
2.52
1.42
1.10
0.00

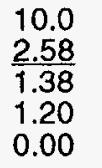

10.6
2.63
1.38
1.25
0.00
6.2

$\frac{2.26}{1.36}$

0.90

0.00

30.4

$\frac{2.26}{1.36}$

0.90

0.00

13.5

$\frac{2.26}{1.36}$
1095

TOTAL MC

553,950

553,950

$2,726,039$

$2,726,039$

$1,215,000$

$1,215,000$

YTD

$, 069,200$
TOTAL Mcf

10.7
250

$\frac{2.50}{1.38}$

1.12

0.00 
VOLUME AND PRICE REPORT

\section{Long Term Imports}

Volumes (MMct/d) \& Prices (\$/MMBTU) of Natural

Gas Imported During the Past 12 Months 04/01/94 - 03/31/95
Long-Term Importer

MICHIGAN CONSOLIDATED GAS COMPANY (WGM Ltd.)

AVG Daily Quantity

Total Price (Noyes. Minnesota)

Commodity Component

Demand Component

Reservation Fee Component

MIDLAND COGENERATION VENTURE1 (1)

(Norcen Energy Resources Ltd.)

AVG Daily Quantity

Total Price (Noyes. Minnesota)

Commodity Component

Demand Component

Reservation Fee Component

MIDLAND COGENERATION VENTURE2

(Shell Canada Limited)

AVG Daily Quantity

Total Price (Noyes. Minnesota)

Commodity Component

Demand Component

Reservation Fee Component

MIDLAND COGENERATION VENTURE3

(Canterra Energy Limited)

AVG Daily Quantity

Total Price (Noyes, Minnesota)

Commodity Component

Demand Component

Reservation Fee Component

MIDLAND COGENERATION VENTURE4

(TransCanada Pipelines Limited)

AVG Daily Quantity

Total Price (Noyes, Minnesota)

Commodity Component

Demand Component

Reservation Fee Component
Auth

1994

32.0

$$
\begin{aligned}
& 28 . \\
& 2.1 \\
& \hline 1.69 \\
& 0.45
\end{aligned}
$$

\begin{tabular}{l}
28.6 \\
2.14 \\
\hline 1.69 \\
0.45 \\
0.00
\end{tabular}

$\begin{array}{rr}6.5 & 6.5 \\ 183 & 1.82\end{array}$

$\frac{1.83}{1.40} \quad \frac{1.82}{1.40}$

$0.43 \quad 0.42$

0.00

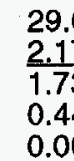

1994
Jun.

$1994 \quad 1994$

Jul.

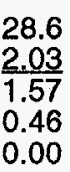

28.6
1.89
1.44
0.45
0.00

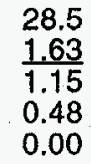
28.5
1.55
$\frac{1.55}{1.09}$
0.46

0.00

26.8
1.82
1.32
0.50
0.00

\begin{tabular}{l}
29.0 \\
1.80 \\
\hline 1.37 \\
0.43 \\
0.00
\end{tabular}

29.8
174

$\frac{1.74}{1.30}$

1.30
0.44

0.00

1.57

$\frac{1.57}{1.07}$

0.50

$\frac{1.53}{1.08}$

0.45

0.00

15.0

$\begin{array}{ll}15.0 & 15.0 \\ \frac{1.77}{1.36} & 1.75 \\ 0.41 & 0.36 \\ 0.00 & 0.00\end{array}$

15.0
1.75
1.36
0.39
0.00

\begin{tabular}{l}
15.0 \\
1.77 \\
\hline 1.36 \\
0.41 \\
0.00
\end{tabular}

15.0
1.74
1.34
0.40
0.00

15.0
1.74
1.34
0.40
0.00

$\begin{array}{ll}1.0 & 15.0 \\ 1.34 & 1.7 \\ 1.40 & 0.43 \\ 0.00 & 0.00\end{array}$

15.0
1.75
1.33
0.42

15.0
173

$\frac{1.73}{1.32}$

0.41

0.41
0.00

1.74

1.32

0.42
0.00

1.73

1.33

0.40
0.00

15.0

\begin{tabular}{ll}
15.0 & 15.0 \\
1.90 & 1.89 \\
\hline 1.50 & 1.50 \\
0.40 & 0.39 \\
0.00 & 0.00
\end{tabular}

\begin{tabular}{l}
15.0 \\
1.90 \\
\hline 1.50 \\
0.40 \\
0.00
\end{tabular}

\begin{tabular}{l}
15.0 \\
1.89 \\
\hline 1.50 \\
0.39 \\
0.00
\end{tabular}

15.0
1.89
1.50
0.39
0.00

15.0

$\frac{1.91}{1.49}$

0.42

\begin{tabular}{l}
15.0 \\
1.89 \\
\hline 1.49 \\
0.40 \\
0.00
\end{tabular}

15.

$15.0 \quad 15$.

$\begin{array}{r}15.0 \\ 1.89 \\ \hline 1.50\end{array}$

0.00

15.0

$\begin{array}{ll}15.0 & 15.0 \\ \frac{1.75}{1.32} & 1.72 \\ 0.43 & 0.42 \\ 0.00 & 0.00\end{array}$

0.00

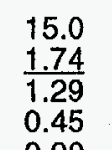

\begin{tabular}{l}
1.74 \\
\hline 1.29 \\
0.45 \\
0.00
\end{tabular}

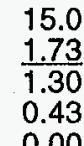

1.73
1.30
0.43

0.00

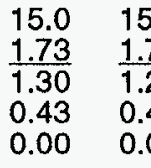

\begin{tabular}{ll}
15.0 & 15.0 \\
1.73 & 1.73 \\
\hline 1.30 & 1.28 \\
0.43 & 0.45 \\
0.00 & 0.00
\end{tabular}

\begin{tabular}{ll}
7.0 & 15.0 \\
73 & 1.7 \\
\hline .28 & 1.28 \\
00 & 0.43 \\
\hline 0 &
\end{tabular}

1.35

0.44

$\frac{1.72}{1.31}$

0.41

900,000

900,000 YTD TOTAL M

$2,631,858$

$2,631,858$

$1,350,000$

$1,350,000$

(1) On November 1, 1994, the daily contract quantity increased from 6.5 to $10.0 \mathrm{MMcf}$ 
Long-Term Importer

MIDLAND COGENERATION VENTURE5 (Poco Petroleums Ltd.)

AVG Daily Quantity

Total Price (Noyes, Minnesota)

Commodity Component

Demand Component

Reservation Fee Component

MIDLAND COGENERATION VENTURE6

(North Canadian Oils, Ltd.)

AVG Daily Quantity

Total Price (Noyes, Minnesota)

Commodity Component

Demand Component

Reservation Fee Component

MINNEGASCO, INC.

(TransCanada Pipelines Limited)

AVG Daily Quantity

Total Price (Noves, Minnesota)

Commodity Component

Demand Component

Reservation Fee Component

N.Y. STATE ELECTRIC \& GAS CO.

(Alberta NE Gas (AEC Oil \& Gas Co.))

AVG Daily Quantity

Total Price (Waddington. NY)

Commodity Component

Demand Component

Reservation Fee Component

N.Y. STATE ELECTRIC \& GAS CO.

(Alberta Northeast Gas (Atcor))

AVG Daily Quantity

Total Price (Waddington, NY)

Commodity Component

Demand Component

Reservation Fee Component

\section{VOLUME AND PRICE REPORT}

\section{Long Term Imports}

Volumes (MMcf/d) \& Prices ( $\$ M M B T U$ ) of Natural

Gas Imported During the Past 12 Months 04/01/94 - 03/31/95

Aिuth. $\quad 1994 \quad 1994 \quad 1994 \quad 1994 \quad 1994 \quad 1994 \quad 1994 \quad 1994 \quad 1994 \quad 1995 \quad 1995 \quad 1995$

Vols. Apr. May. Jun. Jul. Aug. Sep. Oct. Nov. Dec. Jan. Feb. Mar.

1095

TOTAL MCf

TOTAL MCf

15.0

\begin{tabular}{ll}
15.0 & 15.0 \\
\hline 1.95 & $\frac{1.56}{1.34}$ \\
\hline 1.55 & .34 \\
0.00 & 0.22 \\
0.00 & 0.00
\end{tabular}

\begin{tabular}{lll}
15.0 & 15.0 & 15.0 \\
1.97 & 1.96 & 1.97 \\
\hline 1.57 & 1.57 & 1.58 \\
0.40 & 0.39 & 0.39 \\
0.00 & 0.00 & 0.00
\end{tabular}

\begin{tabular}{l}
15.0 \\
1.99 \\
\hline 1.58 \\
0.41 \\
0.00
\end{tabular}

15.0

15.0

15.0

15.0

7.5

10.0

$\frac{1.98}{1.58}$

$0.00 \quad 0.00$

$\begin{array}{lll}0.00 & 0.00 & 0.00\end{array}$

$\begin{array}{ll}0.41 & 0.40 \\ 0.00 & 0.00\end{array}$

1.59

0.00

0.39

0.00

$\frac{2.01}{1.61} \quad \frac{2.52}{1.63} \quad \frac{2.24}{1.64}$

$\begin{array}{lll}0.40 & 0.89 & 0.60\end{array}$

$0.00 \quad 0.00$

10.0

\begin{tabular}{rr}
5.0 & 5.0 \\
2.77 & 2.75 \\
\hline 2.03 & 2.04 \\
0.74 & 0.71 \\
0.00 & 0.00
\end{tabular}

\begin{tabular}{rr}
2.5 & 5.0 \\
3.52 & 2.77 \\
\hline 2.05 & 2.06 \\
1.47 & 0.71 \\
0.00 & 0.00
\end{tabular}

5.0
2.78
2.06
0.72

$\begin{array}{r}5.0 \\ 2.82 \\ \hline 2.06\end{array}$

$\frac{2.82}{2.06}$

0.00

0.00

0.00

0.00

5.0
2.81
2.07
0.74
0.00

5.0
$\frac{2.85}{2.08}$
0.77

5.0

2.81
2.09
0.72

0.00

0.00

0.00

5.0
2.82
2.11
0.71

$\begin{array}{r}5.0 \\ 2.90 \\ \hline 2.11\end{array}$

$\frac{2.90}{2.11}$

0.79

5.0
2.83

$\frac{2.83}{2.12}$

0.71

50.0

\begin{tabular}{ll}
36.9 & 35.0 \\
2.32 & 2.07 \\
\hline 1.68 & 1.56 \\
0.64 & 0.51 \\
0.00 & 0.00
\end{tabular}

\begin{tabular}{l}
45.3 \\
1.74 \\
\hline 1.30 \\
0.44 \\
0.00
\end{tabular}

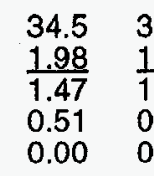

$\begin{array}{lll}34.4 & 34.4 & 41.2\end{array}$

49.1

49.6

$\frac{1.87}{1.36} \quad \frac{1.72}{1.19} \quad \frac{1.63}{1.11}$

$\begin{array}{ll}1.19 & 1.11 \\ 0.53 & 0.52\end{array}$

$\frac{1.96}{1.37}$

$\begin{array}{r}49.6 \\ 2.06 \\ \hline 1.51\end{array}$

49.6
197

49.6

$0.00 \quad 0.00$

$0.00 \quad 0.00$

0.00

0.00

0.00

$\frac{1.97}{1.39}$

$\frac{1.78}{1.14}$

49.5

$\frac{1.71}{1.13}$

1.13
0.58

0.00

985,000

985,000

450,000

$4,459,860$

$4,459,860$

1.3

\begin{tabular}{rr}
1.3 & 1.3 \\
2.17 & 2.24 \\
\hline 1.31 & 1.39 \\
0.86 & 0.85 \\
0.00 & 0.00
\end{tabular}

1.3
2.12
1.27
0.85
0.00

\begin{tabular}{rr}
1.3 & 1.3 \\
2.25 & 2.23 \\
\hline 1.38 & 1.35 \\
0.87 & 0.88
\end{tabular}

1.3
2.14
1.26
0.88
0.00

1.3
1.96
1.07
0.89
0.00

1.3
2.36
1.48
0.88

1.3
2.42
1.50
0.92

3.0

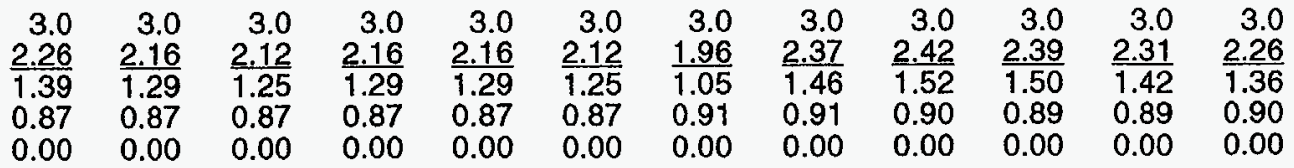




\section{VOLUME AND PRICE REPORT}

\section{Long Term Imports}

Volumes (MMcf/d) \& Prices (\$MMBTU) of Natural

Gas Imported During the Past 12 Months 04/01/94 - 03/31/95
Long-Term Importer

N.Y. STATE ELECTRIC \& GAS CO

(Alberta Northeast Gas (ProGas))

AVG Daily Quantity

Total Price (Waddington. NY)

Commodity Component

Demand Component

Reservation Fee Component

N.Y. STATE ELECTRIC \& GAS CO.

(Alberta Northeast Gas (WGM Ltd. 2))

AVG Daily Quantity

Total Price (Waddington, NY)

Commodity Component

Demand Component

Reservation Fee Component

N.Y. STATE ELECTRIC \& GAS CO.

(Crestar Energy)

Total Price (Grand Island. NY)

Commodity Component

Demand Component

Reservation Fee Component

N.Y. STATE ELECTRIC \& GAS CO. (1)

(Progas Limited)

AVG Daily Quantity

Total Price (Niagara Falls, NY)

Commodity Component

Demand Component

Reservation Fee Component $\begin{array}{lllllllllllll}\text { Auth. } & 1994 & 1994 & 1994 & 1994 & 1994 & 1994 & 1994 & 1994 & 1994 & 1995 & 1995 & 1995\end{array}$

Vols. Apr. Mav. Jun. Jul. Aug. Sep. Oct. Nov. Dec. Jan. Feb. Mar.

5.9

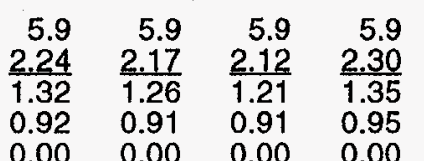

$\begin{array}{llll}0.00 & 0.00 & 0.00 & 0.00\end{array}$

$\begin{array}{rr}5.9 & 5.9 \\ 2.27 & 2.12 \\ 1.31 & 1.21 \\ 0.96 & 0.91 \\ 0.00 & 0.00\end{array}$

5.9
1.96
0.99
0.97
0.00

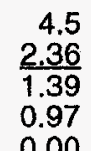

5.9
2.42
1.44 $\frac{2.42}{1.44}$

$\begin{array}{rrrr}6.8 & 6.8 & 6.8 & 6.8 \\ \frac{2.24}{1.37} & \frac{2.17}{1.30} & \frac{2.12}{1.25} & \frac{2.17}{1.30} \\ 0.87 & 0.87 & 0.87 & 0.87 \\ 0.00 & 0.00 & 0.00 & 0.00\end{array}$

NATIONAL FUEL GAS DISTRIBUTION CORPORATION (Alberta NE Gas (WGM Ltd))

AVG Daily Quantity

Total Price (Niagara Falls, NY)

Commodity Component

Demand Component

Reservation Fee Component

\begin{tabular}{r}
8.2 \\
2.46 \\
\hline 1.44 \\
1.02 \\
0.00
\end{tabular}
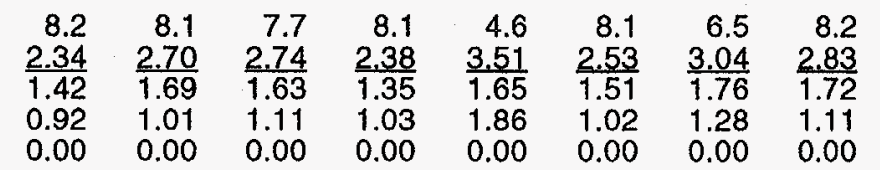

5.8
$\frac{3.23}{1.82}$
1.41

0.00

613,619

613,619

$\begin{array}{llllllllllll}10.0 & 10.0 & 10.0 & 9.9 & 10.0 & 10.0 & 10.0 & 10.0 & 10.0 & 10.0 & 10.0 & 10.0 \\ \frac{2.24}{1.39} & \frac{2.17}{1.32} & \frac{2.15}{1.28} & \frac{2.25}{1.35} & \frac{2.23}{1.31} & \frac{2.14}{1.23} & \frac{1.94}{1.04} & \frac{2.36}{1.46} & \frac{2.41}{1.52} & \frac{2.37}{1.47} & \frac{2.31}{1.43} & \frac{2.24}{1.36} \\ 0.85 & 0.85 & 0.87 & 0.90 & 0.92 & 0.91 & 0.90 & 0.90 & 0.89 & 0.90 & 0.88 & 0.88 \\ 0.00 & 0.00 & 0.00 & 0.00 & 0.00 & 0.00 & 0.00 & 0.00 & 0.00 & 0.00 & 0.00 & 0.00\end{array}$


VOLUME AND PRICE REPORT

\section{Long Term Imports}

Volumes (MMct/d) \& Prices (\$MMBTU) of Natural

Gas Imported During the Past 12 Months 04/01/94 - 03/31/95
Long-Term importer

Auth

Vols.

$\begin{array}{lllll}1994 & 1994 & 1994 & 1994 & 1994\end{array}$

NATURAL GAS PIPELINE COMPANY OF AMERICA

(WGM Ltd.)

AVG Daily Quantity

Total Price (Noyes, Minnesota)

Commodity Componen

Reservation Fee Component

171.3

NEW ENGLAND POWER COMPANY

(Renaissance Energy Limited)

AVG Daily Quantity

Total Price (Waddington, NY)

Commodity Component

Demand Component

Reservation Fee Component

NEW ENGLAND POWER COMPANY

(Sceptre Resources Limited)

AVG Daily Quantity

Total Price (Waddington, NY)

Commodity Component

Demand Component

Reservation Fee Component

NEW ENGLAND POWER COMPANY

(Talisman Energy Inc.)

AVG Daily Quantity

Total Price (Waddington. NY)

Commodity Component

Demand Component

Reservation Fee Component

20.0

10.0

15.0

(Transwest Energy Inc.)

AVG Daily Quantity

Total Price (Waddíngton, NY)

Commodity Component

Demand Component

Reservation Fee Component
1994

1994

1994

$171.3 \quad 163.3$

$$
\begin{array}{r}
171.3 \\
2.13 \\
\hline 1.56 \\
0.42
\end{array}
$$

2.13
1.56
0.42

$\begin{array}{ll}0.15 & 0.44\end{array}$

ay. Jun

\begin{tabular}{|c|c|c|c|c|c|c|c|c|c|c|c|}
\hline $\begin{array}{l}171.3 \\
2.13 \\
1.56 \\
0.42 \\
0.15\end{array}$ & $\begin{array}{r}163.3 \\
2.25 \\
1.66 \\
0.44 \\
0.15\end{array}$ & $\begin{array}{r}171.0 \\
1.99 \\
1.39 \\
0.45 \\
0.15\end{array}$ & $\begin{array}{r}154.8 \\
2.17 \\
1.53 \\
0.48 \\
0.16\end{array}$ & $\begin{array}{r}161.5 \\
1.99 \\
1.39 \\
0.45 \\
0.15\end{array}$ & $\begin{array}{r}171.3 \\
1.71 \\
1.11 \\
0.45 \\
0.15\end{array}$ & $\begin{array}{r}171.3 \\
1.61 \\
1.03 \\
0.44 \\
0.14\end{array}$ & $\begin{array}{r}163.0 \\
1.94 \\
1.31 \\
0.47 \\
0.16\end{array}$ & $\begin{array}{r}165.3 \\
1.90 \\
1.32 \\
0.43 \\
0.15\end{array}$ & $\begin{array}{r}171.3 \\
1.82 \\
1.23 \\
0.44 \\
0.15\end{array}$ & $\begin{array}{r}171.2 \\
1.66 \\
1.02 \\
0.48 \\
0.16\end{array}$ & $\begin{array}{r}171.2 \\
1.63 \\
1.04 \\
0.44 \\
0.15\end{array}$ \\
\hline $\begin{array}{l}10.4 \\
2.69 \\
2.00 \\
0.69 \\
0.00\end{array}$ & $\begin{array}{l}10.0 \\
2.74 \\
2.05 \\
0.69 \\
0.00\end{array}$ & $\begin{array}{r}0.0 \\
0.00 \\
0.00 \\
0.00 \\
0.00\end{array}$ & $\begin{array}{l}13.3 \\
2.77 \\
2.06 \\
0.71 \\
0.00\end{array}$ & $\begin{array}{r}0.6 \\
2.68 \\
1.98 \\
0.70 \\
0.00\end{array}$ & $\begin{array}{r}0.0 \\
0.00 \\
0.00 \\
0.00 \\
0.00\end{array}$ & $\begin{array}{r}0.0 \\
0.00 \\
0.00 \\
0.00 \\
0.00\end{array}$ & $\begin{array}{r}9.6 \\
2.46 \\
1.75 \\
0.71 \\
0.00\end{array}$ & $\begin{array}{l}10.9 \\
2.48 \\
1.78 \\
0.70 \\
0.00\end{array}$ & $\begin{array}{l}11.0 \\
2.47 \\
1.77 \\
0.70 \\
0.00\end{array}$ & $\begin{array}{l}11.1 \\
2.27 \\
1.57 \\
0.70 \\
0.00\end{array}$ & $\begin{array}{l}11.1 \\
2.24 \\
1.55 \\
0.69 \\
0.00\end{array}$ \\
\hline
\end{tabular}

$\begin{array}{ll}10.8 & 10.2 \\ \frac{2.43}{1.61} & \frac{2}{1.57} \\ 0.82 & 0.84 \\ 0.00 & 0.00\end{array}$

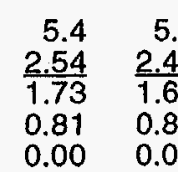

$\begin{array}{lll}0.2 & 0.0 & 0.0\end{array}$

$\frac{2.37}{1.53}$

$\begin{array}{ll}1.53 & 0.00\end{array}$

0.00
0.00

0.00

\begin{tabular}{r}
0.0 \\
0.00 \\
\hline 0.00 \\
0.00
\end{tabular}

5.2

7.5
2.5

1.41

0.85
0.00

$\frac{2.35}{1.48}$

0.87
0.00

$\frac{2.25}{1.40}$

\begin{tabular}{l}
12.8 \\
2.04 \\
\hline 1.18 \\
0.86
\end{tabular}

\begin{tabular}{l}
14.5 \\
1.98 \\
\hline 1.13
\end{tabular}

\begin{tabular}{l}
14.7 \\
1.83 \\
\hline 0.97
\end{tabular}

\begin{tabular}{l}
14.8 \\
1.62 \\
\hline 0.76
\end{tabular}

0.86

14.8

$\frac{1.73}{0.88}$

0.85

0.00
0.0
0.00
0.00
0.00
0.00

0.4
2.39
1.56
0.83

2.6
2.61
1.76

3.1
2.30
1.43
0.87
0.00

4.2
$\frac{2.23}{1.38}$
0.85
0.00

\begin{tabular}{r}
6.4 \\
2.34 \\
\hline 1.50 \\
0.84
\end{tabular}

7.3
2.41
1.58
0.83
0.00

7.3
2.45
1.61
0.84
0.00

\begin{tabular}{r}
7.4 \\
2.42 \\
\hline 1.58 \\
0.84
\end{tabular}

7.4
2.44
1.60
0.84

0.00

0.00

0.00
994,786

994,786

$1,326,380$

$1,326,380$

63,190

663,190
YTTD

TOTAL MCI

$15,412,422$

$15,412,422$

$\begin{array}{rrrrrrrrrrrr}6.3 & 2.8 & 7.7 & 0.0 & 3.9 & 5.8 & 6.4 & 0.0 & 0.0 & 0.0 & 0.0 & 0.0 \\ \frac{3.03}{2.35} & \frac{2.96}{2.28} & \frac{2.69}{2.01} & \frac{0.00}{0.00} & \frac{2.52}{1.83} & \frac{2.19}{1.48} & \frac{2.10}{1.39} & \frac{0.00}{0.00} & \frac{0.00}{0.00} & \frac{0.00}{0.00} & \frac{0.00}{0.00} & \frac{0.00}{0.00} \\ 0.68 & 0.68 & 0.68 & 0.00 & 0.69 & 0.71 & 0.71 & 0.00 & 0.00 & 0.00 & 0.00 & 0.00 \\ 0.00 & 0.00 & 0.00 & 0.00 & 0.00 & 0.00 & 0.00 & 0.00 & 0.00 & 0.00 & 0.00 & 0.00\end{array}$




\section{VOLUME AND PRICE REPORT}

\section{Long Term Imports}

Volumes (MMcf/d) \& Prices (\$MMBTU) of Natural

Gas Imported During the Past 12 Months 04/01/94 - 03/31/95

\section{Long-Term Importer}

NEW JERSEY NATURAL GAS COMPANY (Alberta NE Gas (AEC Oil \& Gas Company) AVG Daily Quantity

Total Price (Waddington. NY)

Commodity Component

Demand Component

Reservation Fee Component

NEW JERSEY NATURAL GAS COMPANY (Alberta Northeast Gas (Atcor))

AVG Daily Quantity

Total Price (Waddington. NY)

Commodity Component

Demand Component

Reservation Fee Component

NEW JERSEY NATURAL GAS COMPANY

(Alberta Northeast Gas (ProGas))

AVG Daily Quantity

Total Price (Waddington, NY)

Commodity Componen

Demand Component

Reservation Fee Component

NEW JERSEY NATURAL GAS COMPANY (Alberta Northeast Gas (WGM Ltd. 1))

AVG Daily Quantity

Total Price (Waddington. NY)

Commodity Component

Demand Component

Reservation Fee Component

NEW JERSEY NATURAL GAS COMPANY (Alberta Northeast Gas (WGM Ltd. 2))

AVG Daily Quantity

Total Price (Waddington, NY)

Commodity Component

Demand Component

Reservation Fee Component
Auth.

Vols.

1994
Apr

19941994

1994

$1994 \quad 1994$

1994

1994

1.9

$$
\begin{array}{ll}
1.9 & \\
\frac{2.17}{1.31} & 2 \\
0.86 & 0.38 \\
0.00 & 0.00
\end{array}
$$

1.9
2.24
1.39
0.85
0.00

1.9
2.12
1.27
0.85
0.00

1.9
2.25
1.38
0.87
0.00
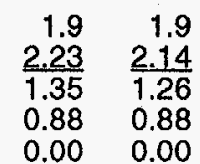

\begin{tabular}{r}
1.9 \\
1.96 \\
\hline 1.07 \\
0.89 \\
0.00
\end{tabular}
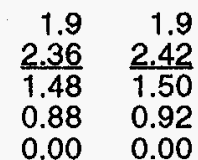

1.9
2.38
1.49
0.89
0.00

1.9
2.31
1.44
0.87
0.00

\begin{tabular}{r}
1.9 \\
2.26 \\
\hline 1.36 \\
0.90 \\
0.00
\end{tabular}

3.0

$$
\begin{array}{rr}
3.0 & 3.0 \\
2.26 & 2.16 \\
\hline 1.39 & 1.29 \\
0.87 & 0.87 \\
0.00 & 0.00
\end{array}
$$

3.0
2.12
1.25

3.0
2.16
1.29

0.87

0.00

1.29
0.87

3.0
2.16
1.29

2.16
1.29
0.87

3.0

\begin{tabular}{r}
3.0 \\
1.96 \\
\hline 1.05 \\
0.91
\end{tabular}

3.0

$\frac{2.37}{1.46}$

0.91

3.0
2.42
1.52

2.42
1.52
0.90

3.0
239

$\frac{2.39}{1.50}$

3.0
$\frac{2.31}{1.42}$

0.89

1.42
0.89

$\frac{2.26}{1.36}$

1.36

0.90

4.6

\begin{tabular}{rrr}
4.6 & 4.6 & \\
2.24 & 2.17 & 2 \\
\hline 1.32 & 1.26 & 1 \\
0.92 & 0.91 & 0.9 \\
0.00 & 0.00 & 0.0
\end{tabular}

4.6
2.12
1.21
0.91
0.00

$\begin{array}{rr}4.6 & 4.6 \\ 2.30 & 2.27 \\ 1.35 & 1.31 \\ 0.95 & 0.96 \\ 0.00 & 0.00\end{array}$

4.6
2.27
1.31
0.96
0.00

4.6
2.12
1.21
0.91

4.6

$\frac{1.96}{0.99}$

0.99
0.97

0.00

4.6
36

$\frac{2.36}{1.39}$

1.39
0.97

0.00

4.6
2.42
1.44
0.98

4.6
2.38
1.38

$\frac{2.38}{1.38}$

1.00
0.00

4.6

$\frac{2.32}{1.34}$

0.98

0.00

4.6

$\frac{2.26}{1.27}$

0.99
0.00
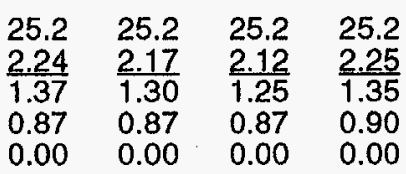

\begin{tabular}{l}
24.2 \\
2.23 \\
\hline 1.31 \\
0.92 \\
0.00
\end{tabular}

25.2
2.14
1.23
0.91

25.2

$25.2 \quad 25$

$\begin{array}{lll}25.2 & 25.2 \quad 25.2\end{array}$

$\frac{2.31}{1.42} \quad \frac{2.26}{1.36}$

$0.00 \quad 0.00$

0.00

0.00

0.92

0.00

$\frac{2.36}{1.45} \quad \frac{2.42}{1.52}$

0.910 .90

$\frac{2.38}{1.47}$

0.91
0.00

0.00

0.00

5.3

\begin{tabular}{rrr}
5.3 & 5.3 & 5.3 \\
2.24 & 2.17 & 2.12 \\
\hline 1.37 & 1.30 & 1.25 \\
0.87 & 0.87 & 0.87 \\
0.00 & 0.00 & 0.00
\end{tabular}

5.3
2.17
1.30
0.87
0.00

5.3
2.22
1.32
0.90
0.00

5.3
2.12
1.25
0.87
0.00

\begin{tabular}{r}
5.3 \\
1.96 \\
\hline 1.04 \\
0.92
\end{tabular}

5.3
236

$\frac{2.36}{1.45} \quad \frac{2.42}{1.52}$

1.451 .52

$\begin{array}{ll}0.00 & 0.00\end{array}$ \begin{tabular}{r}
5.3 \\
2.38 \\
\hline 1.47 \\
0.91 \\
0.00
\end{tabular}

5.3
2.31
1.42
0.89

5.3
226

$\frac{2.26}{1.36}$

0.90

0.00
.31
1.42
0.89
0.00
$2,268,000$

$2,268,000$

472,500

472,500 


\section{VOLUME AND PRICE REPORT}

\section{Long Term Imports}

Volumes (MMcf/d) \& Prices (\$MMBTU) of Natural

Gas Imported During the Past 12 Months 04/01/94 - 03/31/95
Long-Term Importer

NIAGARA MOHAWK POWER CORPORATION (WGM Ltd.)

AVG Daily Quantity

Total Price (Waddington. NY)

Commodity Component

Demand Component

Reservation Fee Component

NORTH JERSEY ENERGY ASSOCIATES

(ProGas Limited)

AVG Daily Quantity

Total Price (Niagara Falls, NY)

Commodity Component

Demand Component

Reservation Fee Component

\section{NORTHEAST ENERGY ASSOCIATES}

(ProGas Limited)

AVG Daily Qu

Total Price (Niagara Falls, NY)

Commodity Component

Demand Component

Reservation Fee Component

NORTHERN MINNESOTA UTILITIES

(WGM Ltd.)

AVG Daily Quantity

Total Price (Noyes: Minnesota)

Commodity Component

Demand Component

Reservation Fee Component

$1994 \quad 1994$

1994

$\begin{array}{lllllll}1994 & 1994 & 1994 & 1994 & 1994 & 1994 & 1995\end{array}$

Vols.

Apr.

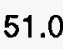

\begin{tabular}{lll}
51.0 & 51.0 & 51.0 \\
2.07 & 2.13 & 1.9 \\
\hline 1.15 & 1.23 & 1.0 \\
0.92 & 0.90 & 0.95 \\
0.00 & 0.00 & 0.00
\end{tabular}

51.0
1.96
1.01
0.95
0.00

51.0
2.09
1.16
0.93
0.00

51.0
1.94

$\frac{1.94}{1.02}$

0.00

24.0

$\begin{array}{lll}21.6 & 21.7 & 2 \\ 2.65 & 2.32 & 2 \\ 1.72 & 1.41 & 1 . \\ 0.93 & 0.91 & 0.9 \\ 0.00 & 0.00 & 0.0\end{array}$

48.0

$\begin{array}{lll}47.9 & 48.0 & 4 \\ \frac{3.10}{2.17} & \frac{3.01}{2.10} & \frac{3}{2} \\ 0.93 & 0.91 & 0 \\ 0.00 & 0.00 & 0.00\end{array}$

10.0

\begin{tabular}{rrr}
9.0 & 6.1 & 3.4 \\
2.32 & 2.55 & 3.18 \\
\hline 1.85 & 1.85 & 1.85 \\
0.47 & 0.70 & 1.33 \\
0.00 & 0.00 & 0.00
\end{tabular}

\begin{tabular}{rr}
1.4 & 0.3 \\
4.99 & 17.24 \\
\hline 1.85 & 1.85 \\
3.14 & 15.39 \\
0.00 & 0.00
\end{tabular}

\begin{tabular}{rr}
3.4 & 2.9 \\
3.17 & 3.35 \\
\hline 1.85 & 1.85 \\
1.32 & 1.50 \\
0.00 & 0.00
\end{tabular}

4.0
2.98
1.85
1.13
0.00

$\frac{2.37}{1.85}$

0.52
0.00

2.6
1.85
0.8

0.81

2.4

$\frac{2.43}{1.85}$

1.85
0.58

NORTHER
(WGM Ltd)

AVG Daily Quantity

Total Price (International Falls MN)

29.8

17.9
2.78
2.08
0.70

0.00
15.6
2.78
2.08
0.70
0.00

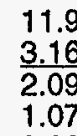

$\frac{3.16}{2.09}$

2.09
1.07

0.00

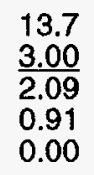

3.00
2.09
0.91

0.00
$12.2 \quad 15.0$

$\frac{3.18}{2.09}$

$\begin{array}{ll}2.09 & 2.08 \\ 1.09 & 0.85\end{array}$

$\begin{array}{ll}1.09 & 0.85 \\ 0.00 & 0.00\end{array}$ \begin{tabular}{l}
16.9 \\
2.33 \\
\hline 1.61 \\
0.72 \\
0.00
\end{tabular}

\begin{tabular}{l}
18.2 \\
2.28 \\
\hline 1.66 \\
0.62 \\
0.00
\end{tabular}
18.7
2.14
1.60
0.54
0.00

20.5
2.01
1.42
0.59

\begin{tabular}{l}
18.8 \\
1.98 \\
\hline 1.40 \\
0.58 \\
0.00
\end{tabular} $\frac{2.97}{1.85}$

1.12

0.00

1095 IOTAL MCf

$4,580,098$

$4,580,098$

$1,950,440$

$1,950,440$

1.49

48.0

$\frac{3.13}{2.17}$

0.96

0.00

523,639

523,639

$1,736,950$

$1,736,950$

(1) This import contract was previously shown as imports made by Inter-City Minnesola Pipelines, Ltd. - 1 . 


\section{VOLUME AND PRICE REPORT}

\section{LON Term Imports}

Volumes (MMcf/d) \& Prices (\$MMBTU) of Natural

Gas Imported During the Past 12 Months 04/01/94 - 03/31/95

Long-Term Importer

Auth.

Vols. Apr. May. Jun 1994

1994

19941994

19941994

NORTHERN MINNESOTA UTILITIES - WESTERN MARKET (1)

(WGM Lid.)

AVG Daily Quantity

Total Price (Warroad, Minnesota)

1.5

\begin{tabular}{rr}
0.8 & 0.5 \\
2.67 & 3.12 \\
\hline 1.91 & 1.91 \\
0.76 & 1.21 \\
0.00 & 0.00
\end{tabular}

0.4
$\frac{3.66}{1.91}$
1.75
0.00

$\begin{array}{rr}0.3 & 0.4 \\ \frac{3.78}{1.91} & \frac{3.49}{1.91} \\ 1.87 & 1.58 \\ 0.00 & 0.00\end{array}$

\begin{tabular}{r}
0.4 \\
3.49 \\
\hline 1.91 \\
1.58
\end{tabular}

Reservation Fee Component

NORTHERN MINNESOTA UTILITIES/CITY OF WARROAD

(WGM Ltd.)

AVG Daily Quantity

Total Price (Warroad, Minnesota)

Commodity Component

Demand Component

Reservation Fee Component

$\begin{array}{rrrrrrrrrrrr}0.2 & 0.1 & 0.1 & 0.1 & 0.1 & 0.1 & 0.2 & 0.3 & 0.5 & 0.6 & 0.6 & 0.4 \\ \frac{3.67}{1.91} & \frac{5.00}{1.91} & \frac{7.93}{1.91} & \frac{7.99}{1.91} & \frac{8.42}{1.91} & \frac{7.00}{1.91} & \frac{4.27}{1.91} & \frac{3.18}{1.91} & \frac{2.76}{1.91} & \frac{2.65}{1.91} & \frac{2.72}{1.91} & \frac{2.88}{1.91} \\ 1.76 & 3.09 & 6.02 & 6.08 & 6.51 & 5.09 & 2.36 & 1.27 & 0.85 & 0.74 & 0.81 & 0.97 \\ 0.00 & 0.00 & 0.00 & 0.00 & 0.00 & 0.00 & 0.00 & 0.00 & 0.00 & 0.00 & 0.00 & 0.00\end{array}$

NORTHERN STATES POWER COMPANY

(Amoco Canada Petroleum Company Ltd.)

AVG Daily Quantity

Total Price (Noyes, Minnesota)

Commodity Component

Demand Component

Reservation Fee Component

NORTHERN STATES POWER COMPANY

(Canadian Occidental Petroleum Co. Ltd.)

AVG Daily Quantity

Total Price (Noyes, Minnesota)

$\begin{array}{rrrr}7.4 & 7.5 & 7.3 & 7.5 \\ 2.15 & 2.02 & 2.02 & 1.93 \\ 1.69 & 1.55 & 1.50 & 1.50 \\ 0.46 & 0.47 & 0.52 & 0.43 \\ 0.00 & 0.00 & 0.00 & 0.00\end{array}$

\begin{tabular}{ll}
7.5 & 7.4 \\
1.93 & 1.94 \\
\hline 1.50 & 1.50 \\
0.43 & 0.44 \\
0.00 & 0.00
\end{tabular}

\begin{tabular}{r}
0.4 \\
3.53 \\
\hline 1.91 \\
1.62 \\
0.00
\end{tabular}

\begin{tabular}{r}
0.5 \\
3.19 \\
\hline 1.91 \\
1.28
\end{tabular}

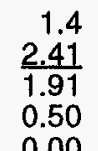

1.8
2.25
1.91
0.34

2.1
$\frac{2.22}{1.91}$

$\frac{2.2}{1.23}$

$\frac{2.34}{1.9}$

$0.32 \quad 0.43$

Demand Component

Reservation Fee Component

NORTHERN STATES POWER COMPANY (ProGas Limited)

AVG Daily Quantity

Total Price (Noyes, Minnesota)

Commodity Component

Reservation Fee Component

\begin{tabular}{rrrrrrr}
7.4 & 7.4 & 7.3 & 7.5 & 7.5 & 7.5 & 4.4 \\
2.24 & 2.19 & 2.04 & 2.06 & $\frac{2.01}{1.51}$ & $\frac{2.07}{1.60}$ & $\frac{2.11}{1.34}$ \\
\hline 1.78 & 1.74 & 1.57 & 1.61 & 1.56 & .47 & 0.77 \\
0.46 & 0.45 & 0.47 & 0.45 & 0.45 & 0.47 & 0.00
\end{tabular}
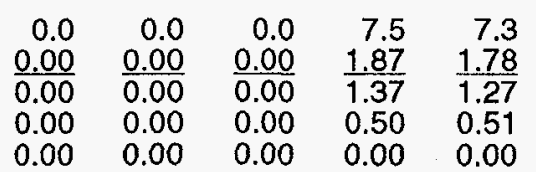
VOLUME AND PRICE REPORT

\section{Long Term Imports}

Volumes (MMcf/d) \& Prices (\$MMBTU) of Natural

Gas Imported During the Past 12 Months 04/01/94 - 03/31/95
Long-Term Importer

Auth. $1994 \quad 1994 \quad 1994$

Vols. Apr. May, Jun.
1994.
Jul.

1994
Aug.
1994

1994
Oct.
1994 Nov. $\begin{array}{llll}1994 & 1995 & 1995 & 1995 \\ \text { Jan } & \text { Feb. } & \text { Mar }\end{array}$

NORTHERN STATES POWER COMPANY (MINNESOTA)

(Amoco Canada Pet. Co. Ltd.)

AVG Daily Quantity

Total Price (Neyes. Minnesota)

Commodity Component

Demand Component

16.0

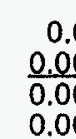

\begin{tabular}{r}
0.0 \\
0.00 \\
\hline 0.00 \\
0.00 \\
0.00
\end{tabular}

\begin{tabular}{rr}
0.0 & 0.0 \\
0.00 & 0.00 \\
\hline 0.00 & 0.00 \\
0.00 & 0.00 \\
0.00 & 0.00
\end{tabular}

0.0
0.00
0.00
0.00
0.00

$\begin{array}{r}0.0 \\ 0.00 \\ \hline .00\end{array}$

$\frac{0.00}{0.00}$

0.0
0.00
0.00
0.00

0.00
0.00

0.00

0.0

$\frac{0.00}{0.00}$

15.7

$\frac{15.7}{1.63}$

0.00
0.00

0.00

\begin{tabular}{l}
15.9 \\
1.75 \\
\hline 1.25 \\
0.50 \\
0.00
\end{tabular}

15.9

$\frac{1.62}{1.14}$

$\begin{array}{r}15.9 \\ 1.42 \\ \hline 0.88\end{array}$

Reservation Fee Component

NORTHERN STATES POWER COMPANY (MINNESOTA)

(WGM Ltd.)

AVG Daily Quantity

Total Price (Noyes. Minnesota)

19.4

0.0

Commodity Component

Demand Component

Reservation Fee Component

NORTHWEST NATURAL GAS COMPANY

(Amoco Canada Petroleum Company Ltd.)

AVG Daily Quantity

Total Price (Sumas. Washington)

Commodity Component

Demand Component

Reservation Fee Component

NORTHWEST NATURAL GAS COMPANY

(CanWest Gas Supply)

AVG Daily Quantity

Total Price (Sumas, Washington)

Commodity Component

Demand Component

Reservation Fee Component

$\begin{array}{rrr}0.0 & 0.0 & 0.0 \\ 0.00 & 0.00 & 0.00 \\ 0.00 & 0.00 & 0.00 \\ 0.00 & 0.00 & 0.00 \\ 0.00 & 0.00 & 0.00\end{array}$

8.0

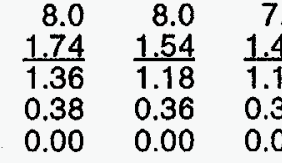

7.9
1.48
1.11
0.37
0.00

$\begin{array}{r}8.0 \\ \quad 1.49 \\ \hline 1.13\end{array}$

$\frac{1.49}{1.13}$
0.36

0.36
0.00

$\begin{array}{rr}0.0 & 0.0 \\ 0.00 & 0.00\end{array}$

0.00

0.00
0.00

0.0
0.00

19.2

0.00

0.00

$0.54 \quad 0.49$

NORTHWEST NATURAL GAS COMPANY

(Poco Petroleums Limited)

AVG Daily Quantity

Total Price (Eastport, Idaho)

Commodity Component

Demand Component

Reservation Fee Component

$\begin{array}{llllllllllll}92.0 & 92.0 & 92.0 & 91.8 & 91.8 & 64.8 & 78.9 & 93.4 & 92.3 & 60.8 & 67.1 & 51.5 \\ \frac{1.79}{1.38} & \frac{1.53}{1.14} & \frac{1.50}{1.10} & \frac{1.54}{1.15} & \frac{1.58}{1.19} & \frac{1.82}{1.24} & \frac{1.79}{1.32} & \frac{1.77}{1.37} & \frac{1.76}{1.37} & \frac{1.93}{1.34} & \frac{1.96}{1.34} & \frac{2.02}{1.29} \\ 0.41 & 0.39 & 0.40 & 0.39 & 0.39 & 0.58 & 0.47 & 0.40 & 0.39 & 0.59 & 0.62 & 0.73 \\ 0.00 & 0.00 & 0.00 & 0.00 & 0.00 & 0.00 & 0.00 & 0.00 & 0.00 & 0.00 & 0.00 & 0.00\end{array}$

$\begin{array}{r}0.0 \\ 0.00 \\ \hline 0.00\end{array}$

0.00
0.00

0.0
0.00

$\frac{0.00}{0.00}$

$\frac{0.00}{0.00}$

\begin{tabular}{rr}
8.0 & 5.4 \\
1.50 & 1.79 \\
\hline 1.14 & 1.23
\end{tabular}

$\frac{1.50}{1.14} \quad \frac{1.79}{1.23}$

$\begin{array}{ll}1.14 & 1.23 \\ 0.36 & 0.56\end{array}$

0.36

0.56
0.00

6.2
1.84
1.36

$\frac{1.84}{1.36}$

0.48
0.00

8.1

$\frac{1.64}{1.18}$

0.46

0.46

\begin{tabular}{l}
19.2 \\
1.83 \\
\hline 1.36 \\
0.47
\end{tabular}

19.2

$\frac{1.75}{1.26}$

19.2
1.52
1.00

$\frac{1.52}{1.00}$

19.2

$\frac{1.47}{0.99}$

0.48

0.00
YTD

TOTAL MCf

$1,427,355$

$1,731,150$ 


\section{VOLUME AND PRICE REPORT}

\section{Long Term Imports}

Volumes (MMct/d) \& Prices (\$MMBTU) of Natural Gas Imported During the Past 12 Months 04/01/94 - 03/31/95
Long-Term Importer

NORTHWEST NATURAL GAS COMPANY

(Summit Resources Limited)

AVG Daily Quantity

Total Price (Eastport, Idaho)

Commodity Component

Demand Component

Reservation Fee Component

NORTHWEST NATURAL GAS COMPANY

(Westcoast Gas Services)

AVG Daily Quantity

Total Price (Eastport, Idaho)

Commodity Component

Demand Component

Reservation Fee Component

\section{NORTHWEST NATURAL GAS COMPANY (1)}

(Westcoast Gas Services)

AVG Daily Quantity

Total Price (Eastport, Idaho)

Commodity Component

Demand Component

Reservation Fee Component

NW ALASKAN PIPELINE CO-Pacific Interstate

(Pan-Alberta Gas Ltd.)

AVG Daily Quantity

Total Price (Eastport, Idaho)

Commodity Component

Demand Component

Reservation Fee Component

\section{Auth}

Apr. May. Jun

8.0

$\begin{array}{ll}1.0 & 3.6 \\ \frac{3.68}{2.01} & 2.25 \\ 1.67 & 1.81 \\ 0.00 & 0.44\end{array}$

23.3

$\begin{array}{rr}3.5 & 9.7 \\ \frac{3.08}{1.54} & \frac{2.20}{1.52} \\ 0.54 & 0.32\end{array}$

1.00

3.8

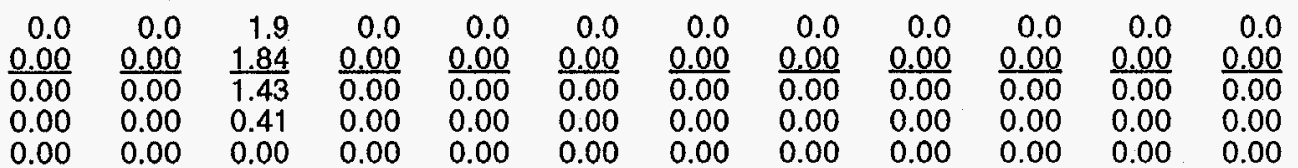

240.0

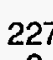 \\ 227}

$\begin{array}{lrr}\frac{2.09}{1.71} & \frac{1.96}{1.61} & \frac{1.92}{1.56} \\ 0.38 & 0.35 & 0.36\end{array}$

0.00

NW ALASKAN PIPELINE CO-Pan-Alberta Gas (1) (2)

(Pan-Alberta Gas Ltd.)

AVG Daily Quantity

Total Price (Port of Morgan, MT)

Commodity Component

Demand Component

Reservation Fee Component

200.0

$\begin{array}{rrrrrrrrrrrr}200.9 & 191.4 & 199.5 & 198.7 & 198.9 & 186.0 & 199.8 & 199.7 & 199.6 & 204.2 & 212.4 & 203.7 \\ \frac{1.93}{1.63} & \frac{2.14}{1.84} & \frac{1.78}{1.48} & \frac{1.69}{1.41} & \frac{1.93}{1.65} & \frac{1.60}{1.29} & \frac{1.52}{1.24} & \frac{1.61}{1.32} & \frac{1.69}{1.41} & \frac{1.69}{1.41} & \frac{1.68}{1.38} & \frac{1.32}{1.03} \\ 0.30 & 0.30 & 0.30 & 0.28 & 0.28 & 0.31 & 0.28 & 0.29 & 0.28 & 0.28 & 0.30 & 0.29 \\ 0.00 & 0.00 & 0.00 & 0.00 & 0.00 & 0.00 & 0.00 & 0.00 & 0.00 & 0.00 & 0.00 & 0.00\end{array}$

1095

IOTAL MC

688,172

688,172

$2,155,076$

$2,155,076$

$\frac{1.35}{0.94}$

0.25

0

$18,593,534$

(1) On November 4, 1994, DOE/FE Order 664-B terminated this contract.
(2). Effective November 1, 1993, the Northern Natural contract was assumed by Pan-Alberta Gas (U.S.) Inc. 


\section{VOLUME AND PRICE REPORT}

\section{Long Term Imports}

Volumes (MMct/d) \& Prices (\$MMBTU) of Natural

Gas Imported During the Past 12 Months 04/01/94 - 03/31/95

Long-Term Importer

$\begin{array}{lllllllllllll}\text { Auth. } & 1994 & 1994 & 1994 & 1994 & 1994 & 1994 & 1994 & 1994 & 1994 & 1995 & 1995 & 1995\end{array}$

NW ALASKAN PIPELINE CO-Pan-Alberta Gas (II)

(Pan-Alberta Gas Ltd.)

AVG Daily Quantity

Total Price (Port of Morgan. MT)

Commodity Componen

Demand Component

Reservation Fee Component

600.0

OCEAN STATE POWER

(ProGas Limited)

AVG Daily Quantity

Total Price (Niagara Falls, NY)

Commodity Componen

Demand Component

Reservation Fee Component

OCEAN STATE POWER II

(ProGas Limited)

AVG Daily Quantity

Total Price (Niagara Falls, NY)

Commodity Componen

Demand Component

Reservation Fee Component
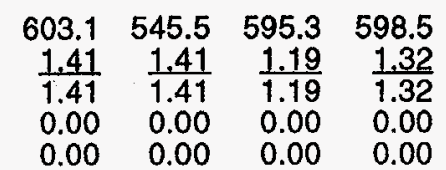

$\begin{array}{r}594.9 \\ 1.19 \\ \hline 1.19\end{array}$

Sep. OC.

ct. Nov.

OCEAN STATE POWER II

(WGM Ltd.)

AVG Daily Quantity

Total Price (Niagara Falls, NY)

Commodity Component

Demand Component

Reservation Fee Component

50.0

$\begin{array}{llllllllllll}51.2 & 51.2 & 51.2 & 51.2 & 51.2 & 51.3 & 50.0 & 50.0 & 50.0 & 50.0 & 50.0 & 50.0 \\ \frac{1.98}{1.06} & \frac{1.99}{1.08} & \frac{1.98}{0.92} & \frac{1.96}{1.05} & \frac{1.95}{1.00} & \frac{1.96}{1.02} & \frac{1.96}{1.02} & \frac{1.96}{1.01} & \frac{1.95}{0.99} & \frac{1.95}{1.00} & \frac{1.96}{1.01} & \frac{1.97}{1.01} \\ 0.92 & 0.91 & 1.06 & 0.91 & 0.95 & 0.94 & 0.94 & 0.95 & 0.96 & 0.95 & 0.95 & 0.96 \\ 0.00 & 0.00 & 0.00 & 0.00 & 0.00 & 0.00 & 0.00 & 0.00 & 0.00 & 0.00 & 0.00 & 0.00\end{array}$

25.0

$\begin{array}{llllllllllll}25.2 & 25.0 & 24.2 & 24.6 & 24.8 & 25.2 & 25.0 & 24.9 & 25.0 & 25.0 & 25.0 & 25.0 \\ \frac{1.99}{1.06} & \frac{1.99}{1.08} & \frac{1.98}{1.06} & \frac{1.96}{1.05} & \frac{1.95}{1.00} & \frac{1.96}{1.02} & \frac{1.96}{1.02} & \frac{1.96}{1.01} & \frac{1.95}{0.99} & \frac{1.95}{1.00} & \frac{1.96}{1.01} & \frac{1.97}{1.01} \\ 0.93 & 0.91 & 0.92 & 0.91 & 0.95 & 0.94 & 0.94 & 0.95 & 0.96 & 0.95 & 0.95 & 0.96 \\ 0.00 & 0.00 & 0.00 & 0.00 & 0.00 & 0.00 & 0.00 & 0.00 & 0.00 & 0.00 & 0.00 & 0.00\end{array}$

25.0

\begin{tabular}{ll}
18.3 & 25.0 \\
1.98 & $\frac{1.99}{1.06}$ \\
\hline 1.06 & 1.13 \\
0.92 & 0.86 \\
0.00 & 0.00
\end{tabular}

24.5

\begin{tabular}{ll}
25.3 & 25.3 \\
1.96 & 1.95 \\
\hline 1.10 & 1.04 \\
0.86 & 0.91 \\
0.00 & 0.00
\end{tabular}

25.5
1.96
1.07
0.89
0.00

\begin{tabular}{l}
25.0 \\
1.96 \\
\hline 1.07 \\
0.89 \\
0.00
\end{tabular}

25.0
1.96
1.06
0.90
0.00

25.0
1.95
1.05
0.90
0.00

25.0

$\frac{1.87}{1.06}$

$25.0 \quad 25.0$

$\frac{1.95}{1.08} \quad \frac{1.97}{1.09}$

$0.00 \quad 0.00$

0.00

0.00

0.00

0.00

$\begin{array}{lll}0.00 & 0.00 & 0.88\end{array}$

ORANGE AND ROCKLAND UTILITIES, INC

(Wascana Energy Marketing)

AVG Daily Quantity

25.0

$\begin{array}{rrrrrrr}0.0 & 0.0 & 0.0 & 11.2 & 25.0 & 25.8 & 24.9 \\ \frac{0.00}{0.00} & \frac{0.00}{0.00} & \frac{0.00}{0.00} & \frac{2.72}{1.70} & \frac{2.56}{1.56} & \frac{2.56}{1.56} & \frac{2.19}{1.22} \\ 0.00 & 0.00 & 0.00 & 0.83 & 0.83 & 0.83 & 0.83 \\ 0.00 & 0.00 & 0.00 & 0.19 & 0.17 & 0.17 & 0.14\end{array}$

24.8
2.48
1.46
0.86
0.16

25.0
2.46
1.47
0.83
0.16

$1.47 \quad \frac{2.41}{1.41}$

\begin{tabular}{l}
2.41 \\
\hline 1.41 \\
0.84
\end{tabular}

$\frac{2.30}{1.23}$

1.23

$\begin{array}{lll}0.16 & 0.93 & 0.84\end{array}$

$\stackrel{1095}{\text { TOTAL Mcf }}$

YTD

TOTAL MC

$55,374,816$

$55,374,816$

$4,500,000$

$4,500,000$

$2,250,000$

$2,250,000$

$2,250,000$

$2,250,000$

Demand Component

Reservation Fee Component

$\begin{array}{lll}0.00 & 0.00 & 0.00\end{array}$

0.17 


\section{VOLUME AND PRICE REPORT}

\section{Long Term Imports}

Volumes (MMct/d) \& Prices (\$MMBTU) of Natural

Gas Imported During the Past 12 Months 04/01/94 - 03/31/95

Long-Term Importer

ORCHARD GAS CORPORATION (1)

(ProGas Limited)

AVG Daily Quantity

Total Price (Waddington. NY)

Commodity Component

Demand Component

Reservation Fee Component

PAN NATIONAL GAS SALES, INC. (2)

(Sonatrading)

AVG Daily Quantity

Total Price (Lake Charles, LA)

Commodity Component

Demand Component

Reservation Fee Component

PAWTUCKET POWER ASSOCIATES

(Home Oil Company Limited)

AVG Daily Quantity

Total Price (Waddington, NY)

Commodity Componen

Demand Component

Reservation Fee Component

PAWTUCKET POWER ASSOCIATES

(Tarragon Oil \& Gas Limited)

AVG Daily Quantity

Total Price (Waddington. NY)

Commodity Component

Demand Component

Reservation Fee Component

PEOPLES NATURAL GAS COMPANY (WGM Ltd.)

AVG Daily Quantity

Total Price (Noyes, Minnesota)

Commodity Component

Demand Component

Reservation Fee Component $\begin{array}{lllllllllllll}\text { Auth. } & 1994 & 1994 & 1994 & 1994 & 1994 & 1994 & 1994 & 1994 & 1994 & 1995 & 1995 & 1995\end{array}$

Vols. Apr. May. Jun. Jul. Aug. Sep. Oct Nov Dec. Jan. Feb. Mar.

25.0

\begin{tabular}{lllllllllllll}
25.0 & 24.6 & 24.0 & 25.0 & 20.6 & 24.8 & 18.8 & 18.8 & 21.2 & 22.1 & 21.3 & 18.8 \\
2.86 & 2.89 & 2.92 & 2.81 & 2.78 & 2.89 & 2.95 & 2.92 & 2.71 & $\frac{2.81}{2.85}$ & $\frac{2.91}{1.87}$ & $\frac{2.93}{1.90}$ \\
\hline 1.94 & 1.96 & 1.97 & 1.90 & 1.87 & 1.96 & 2.02 & 2.00 & 1.90 & 1.83 & 1.87 & 0.98 & 0.98 \\
0.92 & 0.93 & 0.95 & 0.91 & 0.91 & 0.93 & 0.93 & 0.92 & 0.81 & 0.98 & 0.98 & 0.00 & 0.00
\end{tabular}

123.3

\begin{tabular}{|c|c|c|c|c|c|c|c|c|c|c|c|}
\hline $\begin{array}{r}163.8 \\
1.75 \\
1.75 \\
0.00 \\
0.00\end{array}$ & $\begin{array}{r}0.0 \\
0.00 \\
0.00 \\
0.00 \\
0.00\end{array}$ & $\begin{array}{r}0.0 \\
0.00 \\
0.00 \\
0.00 \\
0.00\end{array}$ & $\begin{array}{l}80.4 \\
1.83 \\
1.83 \\
0.00 \\
0.00\end{array}$ & $\begin{array}{r}0.0 \\
0.00 \\
0.00 \\
0.00 \\
0.00\end{array}$ & $\begin{array}{r}0.0 \\
0.00 \\
0.00 \\
0.00 \\
0.00\end{array}$ & $\begin{array}{r}0.0 \\
0.00 \\
0.00 \\
0.00 \\
0.00\end{array}$ & $\begin{array}{r}0.0 \\
0.00 \\
0.00 \\
0.00 \\
0.00\end{array}$ & $\begin{array}{r}0.0 \\
0.00 \\
0.00 \\
0.00 \\
0.00\end{array}$ & $\begin{array}{r}0.0 \\
0.00 \\
0.00 \\
0.00 \\
0.00\end{array}$ & $\begin{array}{r}0.0 \\
0.00 \\
0.00 \\
0.00 \\
0.00\end{array}$ & $\begin{array}{r}0.0 \\
0.00 \\
0.00 \\
0.00 \\
0.00\end{array}$ \\
\hline
\end{tabular}

8.2

\begin{tabular}{|c|c|c|c|c|c|c|c|c|c|c|c|}
\hline $\begin{array}{r}1.9 \\
4.43 \\
1.40\end{array}$ & $\begin{array}{r}4.2 \\
2.76 \\
1.47 \\
1.29 \\
0.00\end{array}$ & $\begin{array}{r}6.6 \\
2.29 \\
1.43 \\
0.86 \\
0.00\end{array}$ & $\begin{array}{r}6.7 \\
2.41 \\
1.52 \\
0.89 \\
0.00\end{array}$ & $\begin{array}{r}6.4 \\
2.39 \\
1.44 \\
0.95 \\
0.00\end{array}$ & $\begin{array}{r}6.7 \\
2.20 \\
1.25\end{array}$ & $\begin{array}{r}6.8 \\
2.61 \\
1.27 \\
1.34 \\
0.00\end{array}$ & $\begin{array}{r}5.4 \\
2.36 \\
1.45\end{array}$ & $\begin{array}{r}5.6 \\
2.57 \\
1.47 \\
1.10 \\
0.00\end{array}$ & $\begin{array}{r}6.8 \\
2.48 \\
1.55 \\
0.93 \\
0.00\end{array}$ & $\begin{array}{r}7.3 \\
2.44 \\
1.48 \\
0.96 \\
0.00\end{array}$ & $\begin{array}{r}6.8 \\
2.44 \\
150\end{array}$ \\
\hline
\end{tabular}

6.0

\begin{tabular}{rr}
1.4 & 3.3 \\
4.43 & 2.76 \\
\hline 1.40 & 1.47 \\
3.03 & 1.29 \\
0.00 & 0.00
\end{tabular}

5.0
2.38
1.52
0.86
0.00

5.2
2.54
1.65
0.89
0.00

4.9
2.54
1.59
0.95
0.00

\begin{tabular}{rr}
5.0 & 5.1 \\
2.32 & 2.77 \\
\hline 1.37 & 1.43 \\
0.95 & 1.34 \\
0.00 & 0.00
\end{tabular}

3.1
2.53
1.62

$\begin{array}{r}4.1 \\ 2.78 \\ \hline 1.68\end{array}$

5.2
2.57
1.64

5.3
2.57
1.61

$\frac{2.57}{1.61}$

0.96

4.9

$\frac{2.58}{1.66}$

0.92

0.00

0.00

0.00

0.00

9.0

\begin{tabular}{rrr}
4.5 & 2.2 & 1.2 \\
3.64 & 5.34 & 9.04 \\
\hline 1.91 & 1.91 & 1.91 \\
1.73 & 3.43 & 7.13 \\
0.00 & 0.00 & 0.00
\end{tabular}

1.2
8.51
1.91
6.60
0.00
1.2
$\frac{8.32}{1.91}$
6.41

0.00 $\begin{array}{lll}0.97 & 1.08 & 1.31\end{array}$ \begin{tabular}{rrr}
1.7 & 3.4 & 2.8 \\
6.70 & $\frac{3.06}{1.91}$ & $\frac{3.36}{1.91}$ \\
\hline .79 & 1.15 & 1.45 \\
0.00 & 0.00 & 0.00
\end{tabular}

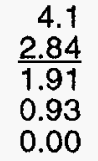

4.1
2.84
1.91
0.93
0.00

4.1

$\begin{array}{r}4.1 \\ 2.99 \\ \hline 1.91\end{array}$

3.0

$\frac{3.22}{1.91}$

$\begin{array}{lll}1.91 & 1.91 & 1.91 \\ 0.97 & 1.08 & 1.31\end{array}$

331,846

(1) Acts as agent for MASSPOWER and Granite State Gas Transmission System.
(2) Tailgate Price. 
VOLUME AND PRICE REPORT

\section{Long Term Imports}

Volumes (MMcf/d) \& Prices (\$MMBTU) of Natural

Gas Imported During the Past 12 Months 04/01/94 - 03/31/95

Long-Term Importer

PEPPERELL POWER ASSOCIATES, L.P. (WGM Ltd.)

AVG Daily Quantity

Total Price (Niagara Falls, NY)

Commodity Component

Demand Component

Reservation Fee Component

POCO PETROLEUM INC.

(IGI Resources, Inc.)

AVG Daily Quantity

Total Price (Sumas. Washington)

Commodity Componen

Demand Component

Reservation Fee Component

PORTAL MUNICIPAL GAS (1)

(SASKENERGY INC.)

AVG Daily Quantity

Total Price (Portal, North Dakota)

Commodity Component

Demand Component

Reservation Fee Component

POWER CITY PARTNERS, L.P.

(Husky Oil Operations Ltd.)

AVG Daily Quantity

Total Price (Massena, New York)

Commodity Component

Demand Component

Reservation Fee Component

PROGAS U.S.A. INC. (2)

(ProGas Limited)

AVG Daily Quantity

Total Price (Noyes, Minnesota)

Commodity Componen

Demand Component

Reservation Fee Component
Âüh

\begin{tabular}{rr}
8.2 & 7.2 \\
1.70 & 1.70 \\
\hline 0.80 & 0.80 \\
0.90 & 0.90 \\
0.00 & 0.00
\end{tabular}

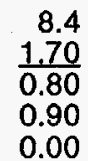

$\begin{array}{r}8.6 \\ 1.70 \\ \hline\end{array}$

$\frac{1.70}{0.80}$

0.90

$\frac{1.70}{0.80}$

0.80
0.90
0.00

$\frac{1.70}{0.80}$

$0.00 \quad 0.00$

$0.00 \quad 0.00$

20.0

\begin{tabular}{lll}
15.0 & 19.9 & 20.1 \\
2.20 & $\frac{1.97}{1.32}$ & $\frac{1.97}{1.32}$ \\
\hline 1.67 & 0.50 & 0.50 \\
0.50 & 0.50 \\
0.03 & 0.15 & 0.15
\end{tabular}

2.3

$\begin{array}{rrr}0.0 & 0.0 & 0.0 \\ \frac{0.00}{0.00} & \frac{0.00}{0.00} & \underline{0.0} \\ 0.00 & 0.00 & 0.00 \\ 0.00 & 0.00 & 0.00\end{array}$

21.0

\begin{tabular}{ll}
15.9 & 15.7 \\
3.35 & 3.28 \\
\hline 1.80 & 1.80 \\
1.55 & 1.48 \\
0.00 & 0.00
\end{tabular}

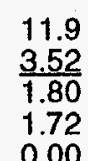

16.6
3.07
1.80
1.27
0.00

16.2
3.18
1.80
1.38
0.00

12.9
3.49
1.80
1.69
0.00

12.3
3.54
1.80
1.74
0.00

\begin{tabular}{l}
17.9 \\
3.11 \\
\hline 1.80 \\
1.31 \\
0.00
\end{tabular}

\begin{tabular}{l}
18.8 \\
$\mathbf{3 . 0 4}$ \\
\hline 1.80 \\
1.24 \\
0.00
\end{tabular}

\begin{tabular}{l}
19.4 \\
3.66 \\
\hline
\end{tabular}

.6 .4
.96
.74

.74

9.6
3.23
1.92
1.31
0.00

85.0

\begin{tabular}{|c|c|c|c|c|c|c|c|c|}
\hline $\begin{array}{l}75.0 \\
2.25 \\
1.50 \\
0.75 \\
0.00\end{array}$ & $\begin{array}{l}75.0 \\
2.10 \\
1.35 \\
0.75 \\
0.00\end{array}$ & $\begin{array}{l}75.0 \\
2.10 \\
1.35 \\
0.75 \\
0.00\end{array}$ & $\begin{array}{l}75.0 \\
2.10 \\
1.35 \\
0.75 \\
0.00\end{array}$ & $\begin{array}{l}75.0 \\
2.10 \\
1.35 \\
0.75 \\
0.00\end{array}$ & $\begin{array}{l}75.0 \\
2.10 \\
1.35 \\
0.75 \\
0.00\end{array}$ & $\begin{array}{l}75.0 \\
2.20 \\
1.45 \\
0.75 \\
0.00\end{array}$ & $\begin{array}{l}68 . \\
2.5 \\
1.6 \\
0.9 \\
0.0\end{array}$ & $\begin{array}{l}42.5 \\
3.05 \\
1.60 \\
1.45 \\
0.00\end{array}$ \\
\hline
\end{tabular}

18.7

$\frac{3.16}{1.92}$

1.24

0.00

1005

TOTAL MC

TOTAL

$$
0
$$

0.00

0.00
0.00

0.00

$1,747,302$

$1,747,302$

6,184

6,184

$1,731,446$

$1,731,446$ 
VOLUME AND PRICE REPORT

\section{Ong Term Imports}

Volumes (MMct/d) \& Prices (\$MMBTU) of Natural Gas Imported During the Past 12 Months 04/01/94 - 03/31/95
Long-Term Importer

PROJECT ORANGE ASSOCIATES, L.P. (1)

(Noranda, Inc.)

AVG Daily Quantity

Total Price (Niagara Falls. NY)

Commodity Component

Demand Component

Reservation Fee Component

PUBLIC SERVICE ELECTRIC \& GAS

(Alberta Northeast Gas (WGM Ltd. 1))

AVG Daily Quantity

Total Price (Waddington. NY)

Commodity Component

Demand Component

Reservation Fee Component

RENAISSANCE ENERGY (U.S.) INC. (2)

(Renaissance Energy Ltd.)

AVG Daily Quantity

Total Price (Port of Morgan, MT)

Commodity Componen

Demand Component

Reservation $\mathrm{Fee}$ Component

Auth.

Vols.

0.0

$\begin{array}{lll}17.5 & 17.7 & 17.4 \\ 2.45 & 2.38 & 2.46 \\ 0.73 & 0.73 & 0.73 \\ 1.72 & 1.65 & 1.73 \\ 0.00 & 0.00 & 0.00\end{array}$

\begin{tabular}{lll}
10.0 & 10.0 & 10.0 \\
2.24 & 2.17 & 2.12 \\
\hline 1.37 & 1.30 & 1.25 \\
0.87 & 0.87 & 0.87
\end{tabular}

$\begin{array}{lll}0.87 & 0.87 & 0.87 \\ 0.00 & 0.00 & 0.00\end{array}$

5.0

\begin{tabular}{|c|c|c|c|c|c|c|c|c|c|c|c|}
\hline $\begin{array}{r}0.0 \\
0.00 \\
\end{array}$ & $\begin{array}{r}0.0 \\
0.00 \\
\end{array}$ & $\begin{array}{r}4.9 \\
1.21 \\
\end{array}$ & $\begin{array}{r}0.0 \\
0.00 \\
\end{array}$ & $\begin{array}{r}0.0 \\
0.00 \\
\end{array}$ & $\begin{array}{r}0.0 \\
0.00 \\
\end{array}$ & $\begin{array}{r}0.0 \\
0.00 \\
\end{array}$ & $\begin{array}{r}0.0 \\
0.00 \\
\end{array}$ & $\begin{array}{r}5.0 \\
1.62 \\
\end{array}$ & $\begin{array}{r}5.1 \\
1.49 \\
\end{array}$ & $\begin{array}{r}5.1 \\
1.24 \\
\end{array}$ & $\begin{array}{r}4.6 \\
1.23 \\
\end{array}$ \\
\hline 0.00 & 0.00 & $\overline{1.21}$ & 0.00 & 0.00 & 0.0 & 0.00 & & & & $\overline{1.24}$ & \\
\hline 0 & 0.0 & $0 . C$ & 0.0 & & & & & & & & \\
\hline & 0.0 & 0.00 & 0.00 & 0.00 & 0.00 & 0.00 & 0.0 & 0.00 & 0.00 & 0.00 & \\
\hline
\end{tabular}

ROCHESTER GAS AND ELECTRIC CORPORATION (Westcoast Gas Services)

AVG Daily Quantity

Total Price (Grand Island. NY)

Commodity Component

Demand Component

Reservation Fee Component

16.0

$\begin{array}{lll}16.0 & 16.0 & 16.0 \\ 2.64 & \frac{2.82}{2.14} & \frac{2.46}{1.78} \\ 1.96 & 2.14 & \\ 0.68 & 0.68 & 0.68 \\ 0.00 & 0.00 & 0.00\end{array}$

16.0
2.65
2.00
0.65
0.00

16.0
2.48

$\frac{2.48}{1.81}$

16.0
2.18
1.49

$\frac{2.18}{1.49}$

16.0
2.11
1.45

14.0
2.29

0.00

0.00

0.00

$\frac{2.20}{1.62}$
0.67

0.00

\begin{tabular}{l}
13.6 \\
2.24 \\
\hline 1.69 \\
0.55 \\
0.00
\end{tabular}

13.6

15.7

$\frac{2.10}{1.54}$

0.56

$\frac{2.00}{1.30}$

0.70

16.0
2.05
1.39
0.66
0.00

\begin{tabular}{lll}
14.5 & 14.5 & 14.6 \\
2.94 & 2.93 & 2.92 \\
\hline 2.08 & 2.10 & 2.06 \\
0.86 & 0.83 & 0.86 \\
0.00 & 0.00 & 0.00
\end{tabular}

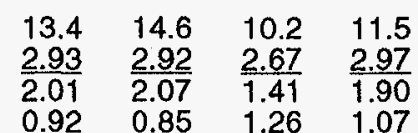

$\begin{array}{llll}2.01 & 2.07 & 1.41 & 1.90 \\ 0.92 & 0.85 & 1.26 & 1.07\end{array}$

0.00
(Shell Canada Limited)

Total Price (Niagara Falls, NY)

Commodity Componen

Reservation Fee Component

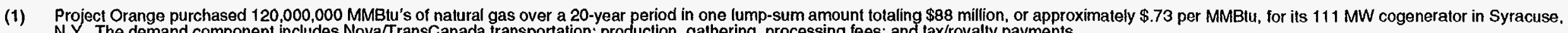
N.Y. The demand component includes Nova/TransCanada transportation; production, gathering, processing fees; and tax/royalty payments

(3) Gas resold to Cogen Energy Technology, Inc. to fuel a cogeneration facility at Castleton-on-Hudson, N.Y. 
VOLUME AND PRICE REPORT

\section{Long Term Imports}

Volumes (MMcf/d) \& Prices (\$MMBTU) of Natural

Gas Imported During the Past 12 Months 04/01/94 - 03/31/95

Long-Term Importer

$\begin{array}{llll}\text { Auth. } & 1994 & 19994 & i 99 \hat{4} \\ \text { Vols. } & \text { Apr. } & \text { May. Jun. }\end{array}$

$1994 \quad 1994 \quad 1994$

1994

Apr.

Jul.

Aug. Sep.

Oct. Nov.

SALMON RESOURCES LTD.-ENRON GAS MARKETING

(Shell Canada Limited)

AVG Daily Quantity

Total Price (Port of Morgan. MT)

Commodity Component

Demand Component

9.8

\begin{tabular}{rr}
9.6 & 9.0 \\
1.61 & 1.57 \\
\hline 1.61 & 1.57 \\
0.00 & 0.00 \\
0.00 & 0.00
\end{tabular}

9.6
1.48
1.48
0.00
0.00

9.6
1.52
1.52

$\begin{array}{rr}9.5 & 8.9\end{array}$

Reservation Fee Component

0.00

0.00

$\frac{1.50}{1.50} \quad \frac{1.44}{1.44}$

$0.00 \quad 0.00$

9.7
1.34
1.34
0.00
0.00

\begin{tabular}{r}
9.7 \\
1.44 \\
\hline 1.44 \\
0.00 \\
0.00
\end{tabular}

9.7
1.52
1.52
0.00

9.7
1.41
1.41

0.00

$0.00 \quad 0.00$

$\frac{1.41}{1.41}$

0.00

1.33

$\frac{1.33}{1.33}$

$0.00 \quad 0.00$

SALMON RESOURCES LTD.-MIDWEST GAS

(Shell Canada Limited)

AVG Daily Quantity

20.5

$\begin{array}{ll}20.2 & 15.2 \\ \frac{1.69}{1.69} & \frac{1.63}{1.63} \\ 0.00 & 0.00\end{array}$

20.2
1.47
1.47

20.2

17.4

Total Price (Port of Morgan, MT)

Commodity Component

Reservation Fee Component

SAN DIEGO GAS \& ELECTRIC COMPANY

(Bow Valley Industries L.td.)

AVG Daily Quantity

Total Price (Eastport, Idaho)

Commodity Component

Demand Component

Reservation Fee Component

SAN DIEGO GAS \& ELECTRIC COMPANY

(Canadian Hunter Marketing Ltd.)

AVG Daily Quantity

Total Price (Eastport, Idaho)

Commodity Component

Demand Component

Reservation Fee Component

SAN DIEGO GAS \& ELECTRIC COMPANY

(Husky Oil Operations Ltd.)

AVG Daily Quantity

Total Price (Eastport, Idaho)

Commodity Component

Demand Component

Reservation Fee Component

\begin{tabular}{|c|c|c|c|c|c|c|c|c|c|c|c|}
\hline $\begin{array}{r}4.9 \\
1.00 \\
1.00\end{array}$ & $\begin{array}{r}5.0 \\
1.00 \\
1.00 \\
0.00 \\
0.00\end{array}$ & $\begin{array}{r}4.9 \\
0.78 \\
0.78 \\
0.00 \\
0.00\end{array}$ & $\begin{array}{r}4.8 \\
0.88 \\
0.88 \\
0.00 \\
0.00\end{array}$ & $\begin{array}{r}4.9 \\
0.82 \\
0.82 \\
0.00 \\
0.00\end{array}$ & $\begin{array}{r}1.3 \\
0.68 \\
0.68 \\
0.00 \\
0.00\end{array}$ & $\begin{array}{r}0.0 \\
0.00 \\
0.00 \\
0.00 \\
0.00\end{array}$ & $\begin{array}{r}0.0 \\
0.00 \\
0.00 \\
0.00 \\
0.00\end{array}$ & $\begin{array}{r}0.0 \\
0.00 \\
0.00 \\
0.00 \\
0.00\end{array}$ & $\begin{array}{r}0.0 \\
0.00 \\
0.00 \\
0.00 \\
0.00\end{array}$ & $\begin{array}{r}0.0 \\
0.00 \\
0.00 \\
0.00 \\
0.00\end{array}$ & $\begin{array}{r}0.0 \\
0.00 \\
0.00 \\
0.00 \\
0.00\end{array}$ \\
\hline
\end{tabular}

19.7

$\begin{array}{ll}19.8 & 19.8 \\ 1.00 & \frac{1.01}{1.01} \\ 0.00 & 0.00\end{array}$

\begin{tabular}{l}
19.5 \\
0.78 \\
\hline 0.78 \\
0.00 \\
0.00
\end{tabular}

19.4

19.4

19.5

$0.00 \quad 0.00$

$\frac{0.82}{0.82}$

0.00

0.68

19.6

19.6

0.00

$\frac{0.46}{0.46}$

0.00
0.00

19.70
0.70
0.00

19.6
0.85

0.85
0.85
0.00

19.7
0.69

$\frac{0.69}{0.69}$

19.7
0.39

$\frac{0.39}{0.39}$

\begin{tabular}{l}
19.7 \\
0.35 \\
\hline 0.35
\end{tabular}

0.35

$1,774,936$

$1,774,936$

21.

$\begin{array}{llll}21.4 & 21.4 & 21.6 & 21.1 \\ 1.00 & \frac{1.00}{1.00} & \underline{0.78} & \underline{0.88} \\ 0.00 & 0.00 & 0.78 & 0.00 \\ 0.00 & 0.00 & 0.00 & 0.00 \\ & & \end{array}$

21.0
0.82
0.82
0.00
0.00

19.8
0.68
0.68
0.00
0.00

20.7
0.46
0.46
0.00
0.00

21.5
0.71
0.71
0.00

21.6

$\frac{0.84}{0.84}$

21.5

0.00

0.00

0

0

$\begin{array}{llll}0.00 & 0.00 & 0.00 & 0.00 \\ 0.00 & 0.00 & 0.00 & 0.00\end{array}$

$0.00 \quad 0.00$

$\begin{array}{ll}0.00 & 0.00\end{array}$

$0.69 \quad 0.40$

0.40

21.5

$\frac{0.49}{0.49}$

0.49

0.00 


\section{VOLUME AND PRICE REPORT}

\section{Long Term Imports}

Volumes (MMcf/d) \& Prices (\$MMBTU) of Natural

Gas Imported During the Past 12 Months 04/01/94 - 03/31/95
Long-Term Importer

SARANAC POWER PARTNERS, L.P.

(Shell Canada Limited)

AVG Daily Quantity

Total Price (Champlain. NY)

Commodity Component

Demand Component

Reservation Fee Component

SELKIRK COGEN PARTNERS, L.P.

(ATCOR Ltd.)

AVG Daily Quantity

Total Price (Waddington, NY)

Commodity Component

Demand Component

Reservation Fee Component

SELKIRK COGEN PARTNERS, L.P.

(Imperial Oil Resources Limited)

AVG Daily Quantity

Total Price (Waddington, NY)

Commodity Component

Demand Component

Reservation Fee Component

SELKIRK COGEN PARTNERS, L.P.

(PanCanadian Petroleum Ltd.)

AVG Daily Quantity

Total Price (Waddington. NY)

Commodity Component

Demand Component

Reservation Fee Component

SELKIRK COGEN PARTNERS, L.P. (1)

(Paramount Resources Ltd.)

AVG Daily Quantity

Total Price (Waddington, NY)

Commodity Component

Demand Component

Reservation Fee Component

\section{Auth}

Auth.
Vols.

$1994 \quad 1994 \quad 1994$

Apr. May. Jun 1994

54.0

\begin{tabular}{rr}
0.9 & 11.7 \\
2.97 & 2.97 \\
\hline 2.08 & 2.1 \\
0.89 & 0.8 \\
0.00 & 0.00
\end{tabular}

17.0

\begin{tabular}{rr}
0.0 & 0.0 \\
0.00 & 0.00 \\
\hline 0.00 & 0.00 \\
0.00 & 0.00 \\
0.00 & 0.00
\end{tabular}

\begin{tabular}{r}
0.0 \\
0.00 \\
\hline 0.00 \\
0.00 \\
0.00
\end{tabular}

0.0
0.00

$\frac{0.00}{0.00}$

0.00

19.0

\begin{tabular}{rrr}
0.0 & 0.0 & 0.0 \\
0.00 & 0.00 & $\underline{0.00}$ \\
\hline 0.00 & 0.00 & 0.00 \\
0.00 & 0.00 & 0.00 \\
0.00 & 0.00 & 0.00
\end{tabular}

\begin{tabular}{r}
0.0 \\
0.00 \\
\hline 0.00 \\
0.00 \\
0.00
\end{tabular}

0.0
0.00

0.00
0.00
0.00
0.00

0.0
0.00
0.00

0.00

0.00
0.00

$\begin{array}{r}0.0 \\ 0.00 \\ \hline .00\end{array}$

$\frac{0.00}{0.00}$

0.00
0.00

1994

1994

1994

1994
Dec.

199

Jan.

(995

Mar.

TOTAL M

$4,123,258$

45.8

3.09

2.24

0.85

0.00

19.0

\begin{tabular}{|c|c|c|c|c|c|c|c|c|c|c|}
\hline $\begin{array}{r}0.0 \\
0.00 \\
0.00 \\
0.00 \\
0.00\end{array}$ & $\begin{array}{r}0.0 \\
0.00 \\
0.00 \\
0.00 \\
0.00\end{array}$ & $\begin{array}{r}0.0 \\
0.00 \\
0.00 \\
0.00 \\
0.00\end{array}$ & $\begin{array}{r}0.0 \\
0.00 \\
0.00 \\
0.00 \\
0.00\end{array}$ & $\begin{array}{r}0.0 \\
0.00 \\
0.00 \\
0.00 \\
0.00\end{array}$ & $\begin{array}{r}0.0 \\
0.00 \\
0.00 \\
0.00 \\
0.00\end{array}$ & $\begin{array}{r}0.0 \\
0.00 \\
0.00 \\
0.00 \\
0.00\end{array}$ & $\begin{array}{l}12.2 \\
2.23 \\
1.16 \\
1.07 \\
0.00\end{array}$ & $\begin{array}{l}13.4 \\
2.30 \\
1.21 \\
1.09 \\
0.00\end{array}$ & $\begin{array}{l}16.9 \\
\frac{2.17}{1.09} \\
1.08 \\
0.00\end{array}$ & $\begin{array}{l}12.3 \\
2.27 \\
1.07 \\
1.20 \\
0.00\end{array}$ \\
\hline
\end{tabular}

23.0

$\begin{array}{llllllllllll}20.4 & 18.3 & 14.6 & 16.1 & 18.5 & 15.6 & 12.8 & 4.9 & 20.1 & 17.7 & 19.2 & 21.0 \\ \frac{2.44}{1.74} & \frac{2.18}{1.49} & \frac{2.26}{1.58} & \frac{2.34}{1.50} & \frac{2.48}{1.62} & \frac{2.45}{1.59} & \frac{2.10}{1.26} & \frac{2.16}{1.28} & \frac{2.29}{1.43} & \frac{2.38}{1.52} & \frac{2.42}{1.53} & \frac{2.26}{1.41} \\ 0.70 & 0.69 & 0.68 & 0.84 & 0.86 & 0.86 & 0.84 & 0.88 & 0.86 & 0.86 & 0.89 & 0.85 \\ 0.00 & 0.00 & 0.00 & 0.00 & 0.00 & 0.00 & 0.00 & 0.00 & 0.00 & 0.00 & 0.00 & 0.00\end{array}$

$1,388,798$

$1,388,798$

2.02

$\begin{array}{lllllll}0.00 & 0.00 & 1.23 & 1.25 & 1.30 & 1.08 & 1.06 \\ .00 & 0.00 & 1.01 & 1.00 & 0.95 & 0.99 & 0.96 \\ .00 & 0.00 & 0.00 & 0.00 & 0.00 & 0.00 & 0.00\end{array}$

$\begin{array}{lllllll}0.00 & 0.00 & 1.23 & 1.25 & 1.30 & 1.08 & 1.06 \\ 0.00 & 0.00 & 1.01 & 1.00 & 0.95 & 0.99 & 0.96 \\ 0.00 & 0.00 & 0.00 & 0.00 & 0.00 & 0.00 & 0.00\end{array}$

\section{0}

$\frac{0.00}{0.00}$

0.00
0.00

0.0
0.00

$\frac{0.00}{0.00}$

0.00

12.2
2.23
1.16
1.07
0.00

13.4
$\frac{2.30}{1.21}$

1.21
1.09

1.09
0.00

\begin{tabular}{l}
16.9 \\
2.17 \\
\hline 1.09 \\
1.08
\end{tabular}

\begin{tabular}{l}
16.5 \\
2.17 \\
\hline 1.05 \\
1.12
\end{tabular}

18.6

$\frac{2.11}{1.01}$

1.10

$1,560,092$

$1,560,092$

0.00

$1,444,838$

$1,444,838$

$1,738,169$

$1,738,169$

(1) A portion of import volumes are made at Niagara Falls, New York. The volumes imported at Niagara include 230,806 (2nd quarter), 302,127 (3rd quarter), and 199,733 (4th quarter). 


\section{VOLUME AND PRICE REPORT}

\section{Long Term Imports}

Volumes (MMcf/d) \& Prices ( $\$ M M B T U$ ) of Natural

Gas Imported During the Past 12 Months 04/01/94 - 03/31/95
Long-Term Importer

Vols.

SITHE/INDEPENDENCE POWER PARTNERS, L.P.

(Enron Power Services Inc.)

AVG Daily Quantity

Total Price (Grand Island, NY)

Commodity Component

Demand Component

Reservation Fee Component

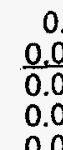

$\begin{array}{rr}0.0 & \\ 0.00 & 0.0 \\ 0.00 & 0.00 \\ 0.00 & 0 \\ 0.00 & 0.00\end{array}$

15.0

\section{(WGM Ltd.)}

SOUTHEASTERN MICHIGAN GAS COMPANY

Total Price (Noyes. Minnesota)

Commodity Component

Demand Component

Reservation Fee Component

SOUTHERN CALIFORNIA EDISON COMPANY

(AEC Oil \& Gas Company)

AVG Daily Quantity

Total Price (Eastport, Idaho)

Commodity Componen

Demand Component

Reservation Fee Component

SOUTHERN CALIFORNIA EDISON COMPANY

(Imperial Oil Resources Limited)

AVG Daily Quantity

Total Price (Eastport, Idaho)

Commodity Component

Demand Component

Reservation Fee Component

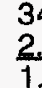

\begin{tabular}{ll}
34.3 & 34.4 \\
$\frac{2.05}{1.99}$ & 1.7 \\
\hline 0.06 & 0.06 \\
0.00 & 0.00
\end{tabular}

$\begin{array}{ll}0.06 & 0.06 \\ 0.00 & 0.00\end{array}$

$\begin{array}{lll}15.0 & 12.9 & 12.0 \\ 2.66 & 2.84 & 2.67 \\ 2.19 & \frac{2.31}{2.08} \\ 0.47 & 0.53 & 0.59 \\ 0.00 & 0.00 & 0.00\end{array}$

$1994 \quad 1994$

1994

1994

1994

1994
Dec.

4199

1995

May. Jun.

ul.

\section{$0.0 \quad 0.0$}

$\frac{0.00}{0.00}$

0.00
0.00

$\frac{0.00}{0.00}$

0.00
0.00

0.0
0.00
0.00
0.00
0.00

\begin{tabular}{r}
0.0 \\
0.00 \\
\hline 0.00 \\
0.00 \\
0.00
\end{tabular}

0.0
0.00
0.00
0.00
0.00

0.0
0.00
0.00
0.00
0.00

9.7
3.92
1.79
2.13

23.8
2.62
1.78
0.84

28.3
2.98
2.28

2.28
0.70
3.04

2.26

$\begin{array}{llll}0.00 & 0.00 & 0.00 & 0.00\end{array}$

SOUTHERN CALIFORNIA EDISON.COMPANY (Shell Canada Limited)

AVG Daily Quantity

Total Price (Eastport, Idaho)

Commodity Component

Demand Component

Reservation Fee Component

\begin{tabular}{ll}
24.5 & 24.6 \\
2.05 & 1.79 \\
\hline 1.99 & 1.73 \\
0.06 & 0.06 \\
0.00 & 0.00
\end{tabular}

24.7
1.44
1.38
0.06
0.00

\begin{tabular}{l}
24.7 \\
1.60 \\
\hline 1.54 \\
0.06 \\
0.00
\end{tabular}

24.7
1.53
1.47
0.06
0.00

24.7
1.18
1.12
0.06
0.00

\begin{tabular}{l}
24.9 \\
1.12 \\
\hline 1.06 \\
0.06 \\
0.00
\end{tabular}

\begin{tabular}{l}
31.7 \\
1.10 \\
\hline 1.04 \\
0.06
\end{tabular}

31.7
1.26
1.20
0.06
0.00

31.4
1.28
1.22
0.06
0.00

\begin{tabular}{l}
31.8 \\
1.12 \\
\hline 1.06 \\
0.06
\end{tabular}

0.06

\begin{tabular}{l}
31.8 \\
0.84 \\
\hline 0.78 \\
0.06 \\
0.00
\end{tabular}

51.0

\begin{tabular}{|c|c|c|c|c|c|c|c|c|c|c|c|}
\hline $\begin{array}{l}49.0 \\
2.01 \\
1.95 \\
0.06 \\
0.00\end{array}$ & $\begin{array}{l}49.2 \\
1.95 \\
1.89 \\
0.06 \\
0.00\end{array}$ & $\begin{array}{l}49.3 \\
1.21 \\
1.15 \\
0.06 \\
0.00\end{array}$ & $\begin{array}{l}49.3 \\
1.44 \\
1.38 \\
0.06 \\
0.00\end{array}$ & $\begin{array}{l}49.3 \\
1.44 \\
1.38 \\
0.06 \\
0.00\end{array}$ & $\begin{array}{l}49.6 \\
1.14 \\
1.08 \\
0.06 \\
0.00\end{array}$ & $\begin{array}{l}49.8 \\
0.87 \\
0.81 \\
0.06 \\
0.00\end{array}$ & $\begin{array}{l}51.1 \\
1.10 \\
1.04 \\
0.06 \\
0.00\end{array}$ & $\begin{array}{l}50.0 \\
1.24 \\
1.18 \\
0.06 \\
0.00\end{array}$ & $\begin{array}{l}49.5 \\
1.35 \\
1.29 \\
0.06 \\
0.00\end{array}$ & $\begin{array}{l}50.1 \\
1.51 \\
1.45 \\
0.06 \\
0.00\end{array}$ & $\begin{array}{l}50.1 \\
0.94 \\
0.88 \\
0.06 \\
0.00\end{array}$ \\
\hline
\end{tabular}

0.78

3.00
2.28
0.72

0.00

1095 TOTAL MCA

$2,524,922$

$1,209,322$

$1,209,322$

$2.18 \quad 2.44$

$0.49 \quad 0.64$

0.64
0.00

35.1

$\frac{0.78}{0.72}$

0.72
0.06

0.00

$3,143,503$

$3,143,503$

$2,847,048$

$2,847,048$

$4,490,849$

$4,490,849$

YTD

TOTAL MCf

\section{$2,524,922$}

143,503

.. 
VOLUME AND PRICE REPORT

\section{Long Term Imports}

Volumes (MMcf/d) \& Prices (\$MMBTU) of Natural

Gas Imported During the Past 12 Months 04/01/94 - 03/31/95
Long-Term Importer

SOUTHERN CALIFORNIA EDISON COMPANY (WGM Ltd.)

AVG Daily Quantity

Total Price (Eastport, Idaho)

Commodity Component

Demand Component

Reservation Fee Component

SOUTHERN CONNECTICUT GAS

(Alberta NE Gas (AEC Oil \& Gas Company))

AVG Daily Quantity

Total Price (Waddington. NY)

Commodity Component

Demand Component

Reservation Fee Component

\section{SOUTHERN CONNECTICUT GAS}

(Alberta Northeast Gas (Atcor))

AVG Daily Quantity

Total Price (Waddington, NY)

Commodity Componen

Demand Component

Reservation Fee Component

SOUTHERN CONNECTICUT GAS

(Alberta Northeast Gas (ProGas))

AVG Daily Quantity

Total Price (Waddington, NY)

Commodity Component

Demand Component

Reservation Fee Component

SOUTHERN CONNECTICUT GAS

(Alberta Northeast Gas (WGM Ltd. 1))

AVG Daily Quantity

Total Price (Waddington, NY)

Commodity Component

Demand Component

Reservation Fee Component

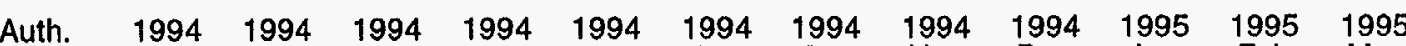

Vols. Apr. Mav. Jun. Jul. Aug. Sep. Oct. Nov. Dec. Jan. Fob. Mar

51.8

$$
\begin{aligned}
& 34.3 \\
& 2.05 \\
& 1.99
\end{aligned}
$$

34.3
2.05
1.99
0.06
0.00

\begin{tabular}{l}
34.4 \\
1.79 \\
\hline 1.73 \\
0.06 \\
0.00
\end{tabular}

34.5
1.44
1.38
0.06
0.00

34.5
1.60
1.54
0.06
0.00

34.5
1.53
1.47
0.06
0.00

34.7

$\frac{1.18}{1.12}$

34.8

54.3

34.5

0.00

2.2

\begin{tabular}{rr}
2.2 & 2.2 \\
2.17 & $\frac{2.24}{1.39}$ \\
\hline 1.31 & 0.86 \\
0.00 & 0.85
\end{tabular}

\begin{tabular}{rrr}
2.2 & 2.2 & 2.2 \\
2.12 & 2.25 & 2.23 \\
\hline 1.27 & 1.38 & 1.35 \\
0.85 & 0.87 & 0.88 \\
0.00 & 0.00 & 0.00
\end{tabular}

\begin{tabular}{r}
2.2 \\
2.14 \\
\hline 1.26 \\
0.88 \\
0.00
\end{tabular}

2.2
1.96
1.07

$0.00 \quad 0.00$

3.7

\begin{tabular}{rr}
3.7 & 3.7 \\
2.26 & 2.16 \\
\hline 1.39 & 1.29 \\
0.87 & 0.87 \\
0.00 & 0.00
\end{tabular}

3.7
2.12
1.25
0.87
0.00

3.7
2.16
1.29
0.87
0.00

3.7
2.16
1.29

$\frac{2.16}{1.29}$

0.87
0.00

\begin{tabular}{rr}
3.7 & 3.7 \\
2.12 & 1.96 \\
\hline
\end{tabular}

$\frac{2.12}{1.25} \quad \frac{1.96}{1.05}$

$\begin{array}{ll}1.25 & 1.05 \\ 0.00 & 0.91\end{array}$

$0.00 \quad 0.00$

3.7
$\frac{2.37}{1.46}$

1.46
0.91

0.00

6.6

\begin{tabular}{rrr}
6.6 & 6.6 & \\
2.24 & 2.17 & 2 \\
\hline 1.32 & 1.26 & 1 \\
0.92 & 0.91 & 0 \\
0.00 & 0.00 & 0.00
\end{tabular}

6.6
2.12
1.21
0.91
0.00

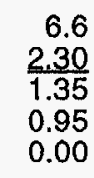

6.6
2.27
1.31

$\frac{2.27}{1.31}$

$\begin{array}{rr}6.6 & 6.6 \\ 2.12 & 196\end{array}$

$\frac{2.12}{1.21} \quad \frac{1.96}{0.99}$

$\begin{array}{ll}1.21 & 0.99 \\ 0.91 & 0.97\end{array}$

6.6
2.36

$\frac{2.36}{1.39}$

1.39
0.97
0.00

0.00

0.00

15.0

\begin{tabular}{ll}
15.0 & 15.0 \\
2.24 & 2.17 \\
\hline 1.37 & 1.30 \\
0.87 & 0.87 \\
0.00 & 0.00
\end{tabular}

15.0
2.12
1.25
0.87
0.00

\begin{tabular}{l}
15.0 \\
2.25 \\
\hline 1.35 \\
0.90 \\
0.00
\end{tabular}

15.0
2.23
1.31
0.92
0.00

\begin{tabular}{l}
15.0 \\
2.14 \\
\hline 1.23 \\
0.91 \\
0.00
\end{tabular}

\begin{tabular}{l}
15.0 \\
1.96 \\
\hline 1.04 \\
0.92 \\
0.00
\end{tabular}

\begin{tabular}{l}
15.0 \\
2.36 \\
\hline 1.45 \\
0.91 \\
0.00
\end{tabular}

22.5
2.42
1.52
0.90
0.00

\begin{tabular}{lll}
22.5 & 22.5 & 22.5 \\
2.38 & 2.31 & 2.26 \\
\hline 1.47 & 1.42 & 1.36 \\
0.91 & 0.89 & 0.90 \\
0.00 & 0.00 & 0.00
\end{tabular}
$2,025,000$

$2,025,000$

YTD

1095
IOTAL MC

TOTAL MCI

$4,889,577$

195,300

195,300

335,700

335,700

594,000

594,000

1.27

0.99

2.5
.26
.36
.90
.00




\section{VOLUME AND PRICE REPORT}

\section{Long Term Imports}

Volumes (MMct/d) \& Prices (\$MMBTU) of Natural

Gas Imported During the Past 12 Months 04/01/94 - 03/31/95
Long-Term Importer

SOUTHERN CONNECTICUT GAS

(Alberta Northeast Gas (WGM Ltd. 2))

AVG Daily Quantity

Total Price (Waddington, NY)

Commodity Component

Demand Component

Reservation Fee Component

ST. LAWRENCE GAS COMPANY INC.

(Niagara Gas Transmission Ltd.)

AVG Daily Quantity

Total Price (Massena. New York)

Commodity Componen

Demand Component

Reservation Fee Component

SUMAS COGENERATION COMPANY, L.P.

(ENCO Gas, Ltd.)

AVG Daily Quantity

Total Price (Sumas. Washington)

Commodity Component

Demand Component

Reservation Fee Component

SUMAS COGENERATION COMPANY, L.P

(Westcoast Gas Services)

AVG Daily Quantity

Total Price (Sumas. Washington)

Commodity Component

Demand Component

Reservation Fee Component

\section{TENASKA GAS COMPANY}

(Husky Oil Operations Ltd.)

AVG Daily Quantity

Total Price (Sumas, Washington)

Commodity Componen

Demand Component

Reservation Fee Component
11.9
2.00
1.60
0.40
0.00

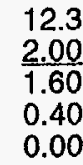

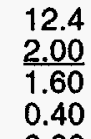

$\frac{2.00}{1.60}$

0.40

0.00

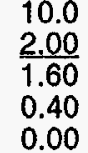

$\frac{2.00}{1.60}$
0.40
0.00
10.0
2.00
1.60
0.40

0.40
0.00

$\begin{array}{rrrrrrrrrrrr}11.1 & 11.1 & 10.3 & 10.0 & 11.9 & 12.2 & 13.2 & 1.1 & 1.9 & 2.7 & 11.6 & 12.1 \\ \frac{2.10}{2.10} & \frac{2.10}{2.10} & \frac{2.10}{2.10} & \frac{2.10}{2.10} & \frac{2.10}{2.10} & \frac{2.10}{2.10} & \frac{2.10}{2.10} & \frac{2.26}{2.26} & \frac{2.26}{2.26} & \frac{2.26}{2.26} & \frac{2.26}{2.26} & \frac{2.26}{2.26} \\ 0.00 & 0.00 & 0.00 & 0.00 & 0.00 & 0.00 & 0.00 & 0.00 & 0.00 & 0.00 & 0.00 & 0.00 \\ 0.00 & 0.00 & 0.00 & 0.00 & 0.00 & 0.00 & 0.00 & 0.00 & 0.00 & 0.00 & 0.00 & 0.00\end{array}$

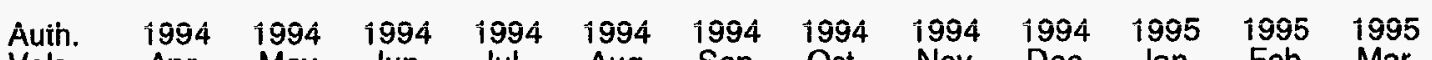

7.5

$\begin{array}{ll}7.5 & 7.5 \\ 2.24 & 2.17 \\ 1.37 & 1.30 \\ 0.87 & 0.87 \\ 0.00 & 0.00\end{array}$

43.0

\begin{tabular}{ll}
16.8 & 12.5 \\
2.54 & 2.54 \\
\hline 2.54 & 2.54 \\
0.00 & 0.00
\end{tabular}

$0.00 \quad 0.00$

7.5
2.12
1.25
0.87
0.00
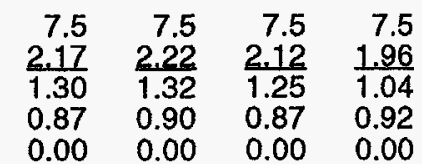

0.0

$\frac{0.00}{0.00}$

0.9100

0.0
0.00

0.00

0.00

$\frac{0.00}{0.00}$

0.00
Vols. Apr. May. Jun. Jul. Aug. Sep. Oct. Nov Dec. Jan. Feb. Mar.

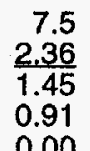

\begin{tabular}{rrrrr}
9.5 & 7.2 & 8.5 & 10.3 & 12.4 \\
2.52 & $\frac{2.48}{2.52}$ & $\frac{2.64}{2.64}$ & $\frac{2.61}{2.61}$ & $\frac{2.35}{2.35}$ \\
\hline 2.52 & 2.48 & 2.64 & 0.60 & 0.00 \\
0.00 & 0.00 & 0.00 & 0.00 & 0.00
\end{tabular}

0.00
1095
TOTAL

TOTAL MCf

TOTAL MC

0

0

783,511

\begin{tabular}{|c|c|}
\hline $\begin{array}{r}9.6 \\
1.66 \\
1.00 \\
0.66 \\
0.00\end{array}$ & $\begin{array}{r}9.6 \\
1.66 \\
1.00 \\
0.66 \\
0.00\end{array}$ \\
\hline
\end{tabular}

7.8
1.70
1.00
0.70
0.00

9.4
1.66
1.00
0.66
0.00

9.6
1.72
1.00
0.72
0.00

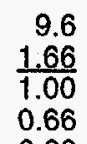

8.

$\begin{array}{ll}0.66 & 0.66 \\ 0.00 & 0.00\end{array}$

0.00

13.0

\begin{tabular}{rrr}
4.0 & 0.0 & 10.0 \\
2.00 & $\underline{0.00}$ & $\frac{2.00}{1.60}$ \\
\hline 2.00 & 0.00 & 0.40 \\
0.00 & 0.00 & 0.40 \\
0.00 & 0.00 & 0.00
\end{tabular}

9.6
1.80
1.08
0.72
0.00

9.6

$\frac{1.80}{1.08}$

0.72
0.00

9.7
$\frac{1.80}{1.08}$
0.72
0.00

9.6

$\frac{1.80}{1.08}$

0.72

0.00

867,171

867,171

881,148

881,148 
VOLUME AND PRICE REPORT

\section{Long Term Imports}

Volumes (MMct/d) \& Prices (\$MMBTU) of Natural Gas Imported During the Past 12 Months 04/01/94 - 03/31/95

Long-Term Importer

TENASKA GAS COMPANY

(Petro-Canada Ltd.)

AVG Daily Quantity

Total Price (Sumas, Washington)

Commodity Component

Demand Component

Reservation Fee Component

\section{TENASKA GAS COMPANY}

(Talisman Energy Inc.)

AVG Daily Quantity

Total Price (Sumas, Washington)

Commodity Component

Demand Component

Reservation Fee Component

TENNESSEE GAS PIPELINE COMPANY

(KannGaz Producers Ltd.)

AVG Daily Quantity

Total Price (Niagara Falls, NY)

Commodity Component

Demand Component

Reservation Fee Component

TENNESSEE GAS PIPELINE COMPANY (1)

(TransCanada Pipelines Limited)

AVG Daily Quantity

Total Price (Niagara Falls. NY)

Commodity Component

Demand Component

Reservation Fee Component

THE MONTANA POWER COMPANY

Canadian-Montana Pipeline Company)

AVG Daily Quantity

Total Price (Whitlash, Montana)

Commodity Component

Demand Component

Reservation Fee Component

(1) Gas imported on behalf of assignees $\begin{array}{lllllllllllll}\text { Auth. } & 1994 & 1994 & 1994 & 1994 & 1994 & 1994 & 1994 & 1994 & 1994 & 1995 & 1995 & 1995\end{array}$

Vols. Apr. May Jun. Jul. Aug. Sep. Oct Nov Dec. Jan. Feb. Mar.

15.0

\begin{tabular}{ll}
14.4 & 0.0 \\
2.15 & 0.00 \\
\hline 1.40 & 0.00 \\
0.75 & 0.00 \\
0.00 & 0.00
\end{tabular}

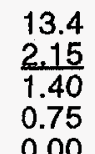

\begin{tabular}{l}
13.1 \\
2.15 \\
\hline 1.40 \\
0.75 \\
0.00
\end{tabular}

13.6

11.5

11.5

$0.00 \quad 0.00$

$0.00 \quad 0.00$

$\frac{2.15}{1.40}$

0.00

1.40
0.75

0.75

$\frac{2.14}{1.40}$

14.4

14.4
2.14
1.40
0.74

14.3

0.74

0.74
0.00

$\frac{2.15}{1.40}$

11.4

$\frac{2.22}{1.44}$

1.44
0.78

18.0

$\begin{array}{rrr}0.0 & 0.0 & 17 \\ 0.00 & \underline{0.00} & \frac{1}{1.08} \\ 0.00 & 0.00 & 1.00 \\ 0.00 & 0.00 & 0 . \\ 0.00 & 0.00 & 0.00\end{array}$

17.3
1.48
1.48
0.00

\begin{tabular}{l}
17.0 \\
1.58 \\
\hline 1.58 \\
0.00 \\
0.00
\end{tabular}

17.1

$\frac{1.54}{1.54}$

17.2
1.43
1.43

17.3

17.3

$0.00 \quad 0.00$

0.00
0.00

1.43

$\frac{1.20}{1.20}$

1.20
0.00

$\frac{1.46}{1.46}$

0.00

17.2

$\frac{1.63}{1.63}$

0.00

16.9
1.45
1.45

0.00

100.0

\begin{tabular}{ll}
50.0 & 50.0 \\
2.82 & 2 \\
\hline 1.94 & 2 \\
0.88 & 0.85 \\
0.00 & 0.00
\end{tabular}

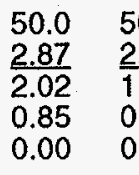

50.0
2.63
1.75
0.88

\begin{tabular}{l}
50.0 \\
2.72 \\
\hline
\end{tabular}

$\frac{2.72}{1.89}$

1.89
0.83
0.00

$50.0 \quad 50.0$

$\frac{2.55}{1.72}$

0.83

$0.83 \quad 0.86$

\begin{tabular}{l}
50.4 \\
2.19 \\
\hline
\end{tabular}

$\frac{2.19}{1.37}$

1.37
0.82
0.00

$\begin{array}{r}0.0 \\ 0.00 \\ \hline\end{array}$

$\frac{0.00}{0.00}$

0.00
0.00

\begin{tabular}{r}
0.0 \\
0.00 \\
\hline 0.00 \\
0.00
\end{tabular}

0.0
0.00

$\frac{0.00}{0.00}$

0.00
0.00

25.0

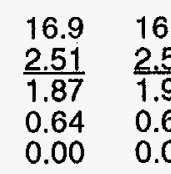

\begin{tabular}{ll}
16.8 & 17.1 \\
2.58 & 2.3 \\
\hline 1.95 & 1.68 \\
0.63 & 0.64 \\
0.00 & 0.00
\end{tabular}

15.0
2.51
1.82
0.69
0.00

\begin{tabular}{l}
15.1 \\
2.33 \\
\hline 1.65 \\
0.68 \\
0.00
\end{tabular}

$\begin{array}{r}0.0 \\ 0.00 \\ \hline\end{array}$

0.0

$\begin{array}{r}0.0 \\ 0.00 \\ \hline\end{array}$

$\begin{array}{r}0.0 \\ 0.00 \\ \hline\end{array}$

0.0

$\frac{0.00}{0.00}$

$\begin{array}{ll}0.00 & 0.00\end{array}$

0.00

0.00

0.00
0.00

50.0

$\begin{array}{rrrrrrr}18.2 & 11.9 & 1.5 & 2.0 & 7.1 & 13.7 & 20.3 \\ \frac{1.41}{1.41} & \frac{1.41}{1.41} & \frac{1.41}{1.41} & \frac{1.41}{1.41} & \frac{1.42}{1.42} & \frac{1.44}{1.44} & \frac{1.45}{1.45} \\ 0.00 & 0.00 & 0.00 & 0.00 & 0.00 & 0.00 & 0.00 \\ 0.00 & 0.00 & 0.00 & 0.00 & 0.00 & 0.00 & 0.00\end{array}$

21.7
1.43
1.43
0.00
0.00

\begin{tabular}{l}
21.9 \\
1.43 \\
\hline 1.43 \\
0.00
\end{tabular}

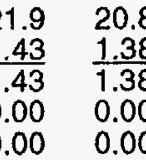

\begin{tabular}{l}
11.9 \\
2.22 \\
\hline 1.44
\end{tabular}

11.2

$\frac{2.22}{1.44}$

1.44
0.78

$0.00 \quad 0.00$

\begin{tabular}{l}
17.3 \\
1.09 \\
\hline
\end{tabular}

$\frac{1.09}{1.09}$

16.8
1.08

1.08

0.00

$0.00 \quad 0.00$

$\begin{array}{r}0.0 \\ 0.00 \\ \hline 0.00\end{array}$

$\frac{0.00}{0.00}$

0.00
0.00

0.0

$\frac{0.00}{0.00}$

0.00

0.00

$\begin{array}{r}0.0 \\ 0.00 \\ \hline 0.00\end{array}$

0.0

$\frac{0.00}{0.00}$

0.00

0.00

0.00

$\begin{array}{ll}21.3 & 21.9 \\ \frac{1.39}{1.39} & 1.39 \\ 0.00 & 0.00 \\ 0.00 & 0.00\end{array}$

$1,917,938$

$1,917,938$

YTD

$1 \mathrm{Q} 95$

TOTAL MC

$1,032,672$

$1,032,672$

$1,528,864$

$1,528,864$

0

0

0

$$
0
$$

$0.00 \quad 0.00$

39
39
00
00




\section{VOLUME AND PRICE REPORT}

\section{Long Term Imports}

Volumes (MMct/d) \& Prices (\$MMBTU) of Natural

Gas Imported During the Past 12 Months 04/01/94 - 03/31/95
Long-Term Importer

THE MONTANA POWER COMPANY

(Canadian-Montana Pipeline Company)

AVG Daily Quantity

Total Price (Port of Del Bonita. MT)

Commodity Component

Demand Component

Reservation Fee Component

THE U.S. GENERAL SERVICES ADMINISTRATION

(Chief Mountain Natural Gas Coop.)

AVG Daily Quantity

Total Price (Babb, Montana)

Commodity Component

Demand Component

Reservation Fee Component

TM STAR FUEL COMPANY

(CanWest Gas Supply)

AVG Daily Quantity

Total Price (Sumas, Washington)

Commodity Component

Demand Component

Reservation Fee Component
Âuth

Vols.
199

$\begin{array}{rrr}0.4 & 0.4 & 0 \\ \frac{1.36}{1.36} & \frac{1.36}{1.36} & 0.0 \\ 0.00 & 0.00 & 0.0 \\ 0.00 & 0.00 & 0.0\end{array}$

0.2

\begin{tabular}{r}
0.0 \\
0.00 \\
\hline 0.00 \\
0.00
\end{tabular}

0.00
0.00

0.00

10.0

$\begin{array}{rr}6.2 & 7.7 \\ \frac{1.90}{1.90} & \frac{1.90}{1.90} \\ 0.00 & 0.00 \\ 0.00 & 0.00\end{array}$

\begin{tabular}{r}
5.9 \\
1.90 \\
\hline 1.90 \\
0.00 \\
0.00
\end{tabular}

8.1
1.90
1.9
0.00
0.00

6.5
1.90
1.90

$\frac{1.90}{1.90}$

1.90
0.00

TRANSCO ENERGY MARKETING COMPANY (1)

(Canstates Gas Marketing)

AVG Daily Quantity

48.4

Total Price (Niagara Falls. NY)

Commodity Componen

Demand Component

Reservation Fee Component

TRANSCO ENERGY MARKETING COMPANY-BG\&E

(Canstates Gas Marketing)

AVG Daily Quantity

Total Price (Niagara Falls, NY)

Commodity Componen

Demand Component

Reservation Fee Component
25.0

\begin{tabular}{rrr}
0.0 & 5.4 & 0.0 \\
0.00 & 2.61 & 0.00 \\
\hline 0.00 & 1.96 & 0.00 \\
0.00 & 0.65 & 0.00 \\
0.00 & 0.00 & 0.00
\end{tabular}

\begin{tabular}{lll}
48.2 & 48.0 & 48.1 \\
2.94 & 2.44 & 2.44 \\
\hline 2.09 & 1.61 & 1.62 \\
0.85 & 0.83 & 0.82 \\
0.00 & 0.00 & 0.00
\end{tabular}

$0.00 \quad 0.00 \quad 0.00$
0.00

$0.62 \quad 0.00$

$\frac{1.90}{1.90}$

$1.90 \quad \frac{1.90}{1.90}$

$0.00 \quad 0.00$

0.00

1994

$1994 \quad 1995 \quad 1995 \quad 1995$

1995
Mar.

1Q95

TOTAL MC

24,866

224

224

764,827

764,827

$\begin{array}{rrrrr}1.90 & 1.90 & \frac{1.97}{1.9} & \frac{1.97}{1.9} & \frac{1.97}{1.97}\end{array}$

$\begin{array}{lll}1.97 & 1.97 & 1.97\end{array}$

$\begin{array}{llllll}0.00 & 0.00 & 0.00 & 0.00 & 0.00\end{array}$

0.00

$1,773,757$

$1,773,757$

2.03

$\frac{2.03}{1.19}$

0.84

0.00

$0.88 \quad 0.86$

0.88
0.00

0.83
0.00

0.88

0.00

\begin{tabular}{rrrr}
8.6 & 0.0 & 0.0 & 0.0 \\
2.48 & 0.00 & 0.00 & 0.00 \\
\hline 1.86 & 0.00 & 0.00 & 0.00 \\
0.62 & 0.00 & 0.00 & 0.00
\end{tabular}

0.0

$\frac{0.00}{0.00}$

10.2
2.24
1.68

$\frac{2.24}{1.68}$

$\begin{array}{lll}24.2 & 24.2 & 22.5\end{array}$

$\frac{2.20}{1.65} \quad \frac{1.96}{1.47}$

22.5
1.95

$0.00 \quad 0.00$

0.00
0.00

0.56
0.00

$\begin{array}{lll}0.55 & 0.49 & 0.49\end{array}$

$\begin{array}{lll}0.00 & 0.00 & 0.00\end{array}$

(1) Gas resold to Hopewell Cogeneration Facility and other TEMCO customers. 


\section{VOLUME AND PRICE REPORT}

\section{Long Term Imports}

Volumes (MMct/d) \& Prices (\$MMBTU) of Natural

Gas Imported During the Past 12 Months 04/01/94 - 03/31/95
Leng-Term Importer

Auth.

Auth.
Vols.
TRANSCO ENERGY MARKETING COMPANY-LILCO

(Canstates Gas Marketing)

AVG Daily Quantity

Total Price (Niagara Falls. NY)

15.0

Demand Component

Reservation Fee Component

TRANSCO ENERGY MARKETING COMPANY-PSE\&G

(Canstates Gas Marketing)

AVG Daily Quantity

Total Price (Niagara Falls. NY)

Commodity Component

Demand Component

Reservation Fee Component

1994

1994

1994
Jul.

1994

1994

1994

1994

1994

$\begin{array}{rrr}0.9 & 0.0 & 0.0 \\ 290 & 0.00 & 0.00\end{array}$

$\frac{2.90}{2.04} \quad \frac{0.00}{0.00} \quad \frac{0.00}{0.00}$

$\begin{array}{lll}0.86 & 0.00 & 0.00 \\ 0.00 & 0.00 & 0.00\end{array}$

$\begin{array}{ll}0.0 & 0.0\end{array}$

$\frac{0.00}{0.00}$

$0.00 \quad 0.00$

0.00
0.00
0.00
0.00

0.0

$\frac{0.00}{0.00}$

12.4

0.00

0.00

$\begin{array}{r}0.0 \\ 0.00 \\ \hline 0.00\end{array}$

0.00

0.00

0.00

14.4
2.59
1.74

1.74

0.85
0.00

14.5

$\frac{2.52}{1.67}$

0.85
0.00

2.33

1.48
0.85

0.00

14.5

$\frac{2.36}{1.51}$

1.51
0.85

0.00

UTILICORP UNITED, INC. (1)

(ProGas Limited)

AVG Daily Quantity

Total Price (Noyes, Minnesota)

Commodity Componen

Demand Component

Reservation Fee Component

2.2
$\frac{2.86}{2.00}$
0.86
0.00

$\begin{array}{ll}10.8 & \\ \frac{2.94}{2.08} & 0.0 \\ 0.86 & 0.0 \\ 0.00 & 0.0\end{array}$

0.0
0.00
0.00
0.00
0.00

1.0
2.82
1.97
0.85

\begin{tabular}{r}
0.0 \\
0.00 \\
\hline 0.00 \\
0.00 \\
0.00
\end{tabular}

8.4

0.0

10.6

0.0

28.2

32.4

26.1
$\frac{2.32}{1.46}$

$\frac{2.36}{1.50} \quad \frac{0.00}{0.00}$

$0.86 \quad 0.00$

$\frac{2.56}{1.70}$
0.86

0.00

0.00

$\frac{2.49}{1.63}$

0.86

$\begin{array}{ll}1.43 & 1.46 \\ 0.86 & 0.86\end{array}$

$0.00 \quad 0.00$

2.7

$\begin{array}{rrr}2.7 & 2.7 & 2.7 \\ \frac{2.07}{1.57} & \frac{2.11}{1.62} & \frac{1.88}{1.36} \\ 0.48 & 0.47 & 0.50 \\ 0.02 & 0.02 & 0.02\end{array}$

7.2

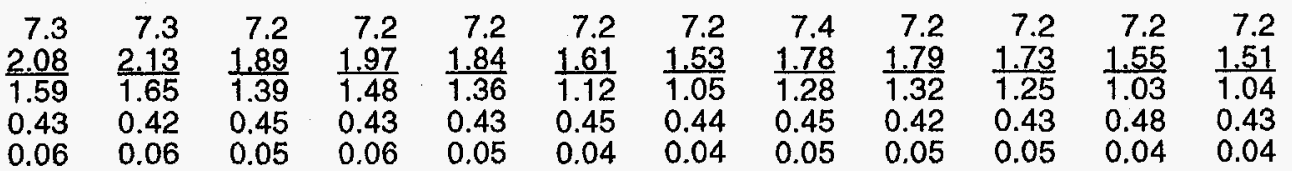

1.0

\begin{tabular}{|c|c|c|}
\hline $\begin{array}{r}1.0 \\
2.24 \\
1.37 \\
0.87 \\
0.00\end{array}$ & $\begin{array}{r}1.0 \\
2.17 \\
1.30 \\
0.87 \\
0.00\end{array}$ & $\begin{array}{r}1.0 \\
2.12 \\
1.25 \\
0.87 \\
0.00\end{array}$ \\
\hline
\end{tabular}

$\begin{array}{rrr}1.0 & 1.0 & 1.0 \\ \frac{2.23}{1.31} & \frac{2.14}{1.23} & \frac{1.96}{1.04} \\ 0.92 & 0.91 & 0.92 \\ 0.00 & 0.00 & 0.00\end{array}$

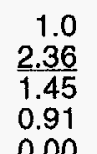

\begin{tabular}{r}
1.0 \\
2.42 \\
\hline 1.52 \\
0.90 \\
0.00
\end{tabular}

1.0
2.38
1.47
0.91
0.00

1.0
2.31
1.42
0.89
0.00

1.0
2.26
1.36
0.90
0.00

89,370

89,370

YTD

TOTAL MCf

$1,304,347$

$1,304,347$

$2,587,925$

$2,587,925$

240,214

240,214

650,622

650,622

Reservation Fee Component

(1) Volumes used by Michigan Gas Uilities.
(2) Volumes used by Michigan Gas Utilities. 
VOLUME AND PRICE REPORT

\section{Long Term Imports}

Volumes (MMcf/d) \& Prices (\$MMBTU) of Natural

Gas Imported During the Past 12 Months 04/01/94 - 03/31/95

Long-Term Importer

VERMONT GAS SYSTEMS INC.

(WGM Ltd.)

AVG Daily Quantity

Total Price (Highgate Spr. VT)

Commodity Component

Demand Component

Reservation Fee Component

WASHINGTON NATURAL GAS COMPANY

(Amoco Canada)

AVG Daily Quantity Washington

Total Price (Sumas, Washington)

Commodity Component

Demand Component
Reservation Fee Component

WASHINGTON NATURAL GAS COMPANY

(Mobil Oil Canada)

Total Price (Sumas. Washington)

Commodity Component

Demand Component

Reservation Fee Component

WASHINGTON NATURAL GAS COMPANY (Poco Petroleums Ltd.)

AVG Daily Quantity

Total Price (Sumas, Washington)

Commodity Component

Demand Component

Reservation Fee Component

WASHINGTON NATURAL GAS COMPANY (Westcoast Gas Services)

AVG Daily Quantity

Total Price (Sumas, Washington)

Commodity Component

Demand Component

Reservation Fee Component

Auth.

19941994

Apr. Mav. Jun.

1994

1994

$1994 \quad 1994$

Vols.

32.0

\begin{tabular}{ll}
20.1 & 13 \\
3.28 & 3.8 \\
\hline 1.85 & 1.73 \\
1.43 & 2.13 \\
0.00 & 0.00
\end{tabular}

24.0

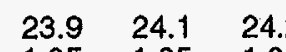

$\begin{array}{lll}1.85 & \frac{1.85}{1.85} & \frac{1.85}{1.85}\end{array}$

$\begin{array}{lll}1.85 & 1.85 & 1.85\end{array}$

$\begin{array}{lll}0.00 & 0.00 & 0.00 \\ 0.00 & 0.00 & 0.00\end{array}$

\begin{tabular}{l}
10.6 \\
4.36 \\
\hline 1.66 \\
2.70 \\
0.00
\end{tabular}

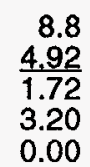

\begin{tabular}{r}
9.2 \\
4.88 \\
\hline 1.81 \\
3.07 \\
0.00
\end{tabular}

\begin{tabular}{l}
11.9 \\
4.35 \\
\hline 1.84 \\
2.51 \\
0.00
\end{tabular}

15.4
3.68

5.4
.68
.87
.81

21.2
2.59
1.34
1.25

0.00

0.00

14.5

$\begin{array}{lll}14.4 & 14.3 & 7.2 \\ 2.23 & \frac{2.16}{1.57} & 2 . \\ 0.52 & 0.52 & 1.55 \\ 0.14 & 0.14 & 0.14\end{array}$

14.4

0.00

0.00

9.6

\begin{tabular}{rrr}
9.7 & 9.7 & 0.0 \\
2.11 & 2.08 & 0.0 \\
\hline 1.68 & 1.68 & 0.0 \\
0.43 & 0.40 & 0.0 \\
0.00 & 0.00 & 0.00
\end{tabular}

\begin{tabular}{ll}
24.2 & 24.2 \\
1.85 & $\frac{1.85}{1.49}$ \\
\hline 1.49 & 0.36 \\
0.36 & 0.36
\end{tabular}

$\begin{array}{ll}0.36 & 0.36 \\ 0.00 & 0.00\end{array}$

\begin{tabular}{l}
24.2 \\
1.85 \\
\hline 1.47 \\
0.38 \\
0.00
\end{tabular}

9.9
2.38
1.48
0.90

25.0
1.58
1.14
0.44

0.44
0.00

$\begin{array}{lll}7.2 & 7.1 \quad 7.1\end{array}$

7.2

55

1.44

$\frac{2.85}{1.53}$

1.49
1.06

8.8
2.48
1.40
0.87
0.21

14.4
1.95

$\frac{1.95}{1.30}$

0.12

9

$0.00 \quad 0.00$ \begin{tabular}{rr}
6.2 & 0.0 \\
2.42 & 0.00 \\
\hline 1.68 & 0.00 \\
0.74 & 0.00 \\
0.00 & 0.00
\end{tabular} \begin{tabular}{r}
9.6 \\
1.80 \\
\hline 1.39 \\
0.41 \\
0.00
\end{tabular}
2.31
0.95
1.36

1.36
0.00

2.21
1.29
0.92

$\frac{2.04}{1.09}$

0.95

0.00

2.15

1.09

1.06

0.00

$\begin{array}{llll}14.4 & 14.3 & 14.4 & 14.2 \\ \frac{2.26}{1.84} & \frac{1.99}{1.65} & \frac{1.59}{1.18} & \frac{1.65}{1.26} \\ 0.42 & 0.34 & 0.41 & 0.39\end{array}$

23.4
$\frac{1.69}{1.27}$
0.42
0.00

25.0
1.46
0.95
0.51

0.51
0.00

25.0

$\frac{1.09}{0.53}$

0.56

25.0

$\frac{1.06}{0.55}$

0.55
0.51
0.00

0.51
0.00

14.4

$\frac{2.13}{1.48}$

1.48
0.52
0.13

$\begin{array}{r}7.7 \\ 2.84 \\ \hline 1.54\end{array}$

2.7
5.67

$\frac{5.67}{1.46}$

1.46
3.00
1.21

0.0

$\frac{0.00}{0.00}$

0.00

0.00

315,000

315,000

$2,250,000$

$2,553,461$
$2,250,000$

$2,553,461$

.

(

$0.27 \quad 1.2$

\begin{tabular}{l}
15.0 \\
1.46 \\
\hline 1.02 \\
0.44 \\
0.00
\end{tabular}

$1,349,999$

$1,349,999$

$\begin{array}{lllll}0.46 & 0.43 & 0.44 & 0.49 & 0.44 \\ 0.00 & 0.00 & 0.00 & 0.00 & 0.00\end{array}$ \begin{tabular}{r}
9.8 \\
1.57 \\
\hline 1.15 \\
0.42 \\
0.00
\end{tabular}

\begin{tabular}{r}
9.6 \\
1.66 \\
\hline 1.26 \\
0.40 \\
0.00
\end{tabular}

\begin{tabular}{l}
10.0 \\
1.41 \\
\hline 1.02 \\
0.39 \\
0.00
\end{tabular}

10.0
1.07
0.61
0.46

10.0

1.04

0.63

0.41
0.00
900,000

900,000 


\section{VOLUME AND PRICE REPORT}

\section{Long Term Imports}

Volumes (MMct/d) \& Prices (\$MMBTU) of Natural

Gas Imported During the Past 12 Months 04/01/94 - 03/31/95
Long-Term Importer

WASHINGTON NATURAL GAS COMPANY

(Westcoast Gas Services)

AVG Daily Quantity

Total Price (Eastport. Idaho)

Commodity Component

Demand Component

Reservation Fee Component

WASHINGTON WATER POWER COMPANY

(AEC Oil \& Gas Company)

AVG Daily Quantity

Total Price (Eastport, Idaho)

Commodity Component

Demand Component

Reservation Fee Component

WASHINGTON WATER POWER COMPANY (Amerada Hess Canada Ltd.)

AVG Daily Quantity

Total Price (Eastport, Idaho)

Commodity Component

Demand Component

Reservation Fee Component

WASHINGTON WATER POWER COMPANY

(PanCanadian Petroleum Limited)

AVG Daily Quantity

Total Price (Eastport, Idaho)

Commodity Component

Demand Component

Reservation Fee Component

WASHINGTON WATER POWER COMPANY (Westcoast Gas Services)

AVG Daily Quantity

Total Price (Eastport, Idaho)

Commodity Component

Demand Component

Reservation Fee Component

\section{Auth. \\ Vo}

$\begin{array}{ll}14.5 & \\ 3.01 & 6.01 \\ 1.65 & 1.65 \\ 1.36 & 4.36 \\ 0.00 & 0.00\end{array}$

25.6

\begin{tabular}{ccc}
0.0 & 0.0 & \\
0.00 & $\underline{0.00}$ & 0.0 \\
\hline 0.00 & 0.00 & 0.0 \\
0.00 & 0.00 & 0.0 \\
0.00 & 0.00 & 0.0
\end{tabular}

16.8

$\begin{array}{lll}10.8 & 10.8 & 10.8 \\ \frac{1.78}{1.39} & \frac{1.84}{1.46} & \frac{1.63}{1.24} \\ 0.39 & 0.38 & 0.39 \\ 0.00 & 0.00 & 0.00\end{array}$

$\begin{array}{lrr}10.0 & 4.2 & 0.0 \\ \frac{1.71}{1.71} & \frac{1.79}{1.79} & 0.00 \\ 0.00 & 0.00 & 0.00 \\ 0.00 & 0.00 & 0.00\end{array}$

19.1

$\begin{array}{lrr}14.7 & 0.5 & 3.7 \\ \frac{1.88}{1.59} & \frac{10.11}{1.64} & \frac{2.54}{1.42} \\ 0.29 & 8.47 & 1.12 \\ 0.00 & 0.00 & 0.00\end{array}$

\begin{tabular}{lr}
.6 & 8.0 \\
& $\frac{3.85}{65}$ \\
\hline
\end{tabular}

$\begin{array}{ll}.65 & 1.32 \\ 36 & 2.53\end{array}$

0.00 $\begin{array}{llll}1994 & 1994 & 1994 & 1994\end{array}$

Aug. Sep, Oct.
1994

Nov.
1994

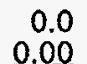

0.00

0.00
0.00

0.00
0.00

\begin{tabular}{l}
27.0 \\
2.20 \\
\hline
\end{tabular}

$\frac{2.20}{1.27}$

0.93
0.00

49.1

$\frac{1.80}{1.15}$

0.65
0.00

\begin{tabular}{l}
65.5 \\
1.49 \\
\hline
\end{tabular}

$\frac{1.49}{1.02}$

0.47
0.00

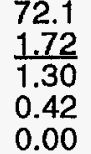

67.7

$\frac{1.85}{1.40}$

0.45

0.00

43.5

$\frac{1.95}{1.19}$

0.76
0.00

$\begin{array}{r}37.2 \\ 1.85 \\ \hline 0.90\end{array}$

0.90
0.95

0.00

0.0
0.00
0.00
0.00
0.00

\begin{tabular}{r}
0.0 \\
0.00 \\
\hline 0.00 \\
0.00 \\
0.00
\end{tabular}

0.0
0.00
0.00
0.00

0.0

0.0

16.4

16.4

16.5

$\begin{array}{r}16.5 \\ 1.98 \\ \hline 1.98\end{array}$

$\begin{array}{r}9.9 \\ 1.98 \\ \hline 1.98\end{array}$

$\begin{array}{r}8.1 \\ 1.98 \\ \hline 1.98\end{array}$

$\begin{array}{llll}0.00 & 0.00 & 0.00\end{array}$

$0.00 \quad 0.00$

0.00

0.00
0.00

1.98
0.00

0.00

0.00
0.00

$0.00 \quad 0.00$

19.0

\begin{tabular}{l}
10.8 \\
1.76 \\
\hline 1.38 \\
0.38 \\
0.00
\end{tabular}

0.00

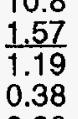

0.00 $\underline{0.96}$

0.31

$\begin{array}{ll}1.27 & 1.18 \\ 0.87 & 0.79\end{array}$

$\begin{array}{ll}0.40 & 0.39 \\ 0.00 & 0.00\end{array}$

$\frac{1.16}{0.84}$

0.32
0.00

0.30
0.00

0.0
0.00
0.00
0.00

0.00
0.00
0.00

$\begin{array}{ll}4.3 & 9.8\end{array}$

$\frac{2.17}{2.17} \frac{1.88}{1.88}$

$0.00 \quad 0.00$

$0.00 \quad 0.00$

10.0

11.0

$\frac{1.67}{1.67}$

1.79
0.00

0.00

0.00

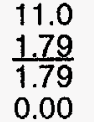

1.79
1.79

0.00

0.00

11.0
1.78
1.78
0.00

$\begin{array}{r}11.0 \\ 1.64 \\ \hline\end{array}$

$\frac{1.64}{1.64}$

0.00
0.00

11.0

$\frac{1.59}{1.59}$

0.00

0.00

\begin{tabular}{rr}
0.0 & 10.6 \\
0.00 & 1.80 \\
\hline 0.00 & 1.26 \\
0.00 & 0.54 \\
0.00 & 0.00
\end{tabular}

\begin{tabular}{l}
15.2 \\
1.59 \\
\hline 1.05 \\
0.54 \\
0.00
\end{tabular}

15.1
1.39
0.88
0.51
0.00

\begin{tabular}{l}
15.0 \\
1.69 \\
\hline 1.19 \\
0.50 \\
0.00
\end{tabular}

\begin{tabular}{l}
15.2 \\
1.80 \\
\hline 1.27 \\
0.53 \\
0.00
\end{tabular}

\begin{tabular}{l}
15.1 \\
1.59 \\
\hline 1.04 \\
0.55 \\
0.00
\end{tabular}

15.1
1.38
0.80
0.58

\begin{tabular}{l}
15.2 \\
1.33 \\
\hline 0.78 \\
0.55
\end{tabular}

0.00
41.1

$\frac{1.68}{0.88}$

0.80

0.00

11.9
$1 \mathrm{Q} 95$

TOTAL MCf

$3,664,684$

$3,664,684$

$1,037,643$

$1,037,643$

$1,071,023$

$1,071,023$

990,020

990,020

$1,361,794$

$1,361,794$

YTD

(

(1)
TOTAL MCf 


\section{VOLUME AND PRICE REPORT}

\section{Long Term Imports}

Volumes (MMct/d) \& Prices (\$MMBTU) of Natural

Gas Imported During the Past 12 Months 04/01/94 - 03/31/95

Long-Term Importer

$1994 \quad 1994 \quad 1994$

1994

Vols.

Apr.

WESTCOAST GAS SERVICES INC. - CIBOLA CORPORATION (Westcoast Gas)

AVG Daily Quantity

Total Price (Port of Morgan. MT)

1.2

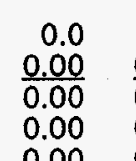

$\begin{array}{rr}0.0 & 0.0 \\ 0.00 & 0.00 \\ 0.00 & 0.00 \\ 0.00 & 0.00\end{array}$

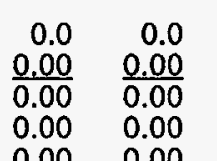

$1994 \quad 1994 \quad 1994$

1994

1994

Commodity Component

0.00

$0.00 \quad 0.00$

Reservation Fee Component

WESTCOAST GAS SERVICES INC. - INTERSTATE POWER CO.

(Westcoast Gas)

Total Price (Port of Morgan. MT)

1.0

\begin{tabular}{rr}
0.0 & 0.0 \\
0.00 & 0.00 \\
\hline 0.00 & 0.00 \\
0.00 & 0.00
\end{tabular}

0.0
0.00
0.00

Commodity Component

Demand Component

Reservation Fee Component

WESTCOAST GAS SERVICES INC. - METRO. UTIL. DIST.

(Westcoast Gas)

AVG Daily Quantity

Total Price (Port of Morgan, MT)

3.7

\begin{tabular}{r}
0.0 \\
0.00 \\
\hline 0.00 \\
0.00
\end{tabular}

$\begin{array}{rrr}0.0 & 0.0 & \\ 0.00 & 0.00 & 0 . \\ 0.00 & 0.00 & 0.0 \\ 0.00 & 0.00 & 0.0 \\ 0.00 & 0.00 & 0.0\end{array}$

\begin{tabular}{r}
0.0 \\
0.00 \\
\hline 0.00 \\
0.00 \\
0.00
\end{tabular}

\begin{tabular}{r}
0.0 \\
0.00 \\
\hline 0.00 \\
0.00
\end{tabular}

0.00

Demand Component
Reservation Fee Component

WESTCOAST GA

AVG Daily Quantity

Total Price (Port of Morgan. MT)

Commodity Component

Demand Component

Reservation Fee Component

6.5

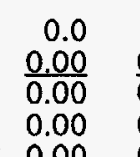

\begin{tabular}{rr}
0.0 & 0.0 \\
0.00 & 0.00 \\
\hline 0.00 & 0.00 \\
0.00 & 0.00 \\
0.00 & 0.00
\end{tabular}

0.0
0.00
0.00
0.00
0.00

$\begin{array}{r}0.0 \\ 0.00 \\ \hline\end{array}$

$\frac{0.00}{0.00}$

0.00

$\begin{array}{ll}0.00 & 0.00 \\ 0.00 & 0.00\end{array}$

\begin{tabular}{rr}
0.0 & 0.0 \\
0.00 & 0.00 \\
\hline 0.00 & 0.00 \\
0.00 & 0.00 \\
0.00 & 0.00
\end{tabular}

\begin{tabular}{r}
0.0 \\
0.00 \\
\hline 0.00 \\
0.00 \\
0.00
\end{tabular}

1.2
$\frac{1.33}{1.33}$
0.00

\begin{tabular}{r}
1.2 \\
1.47 \\
\hline 1.47 \\
0.00
\end{tabular}

$\frac{1.3}{1.34}$

\section{4}

$\frac{1.09}{1.09}$

$\frac{1.09}{1.09}$

1.08

1.08

0.00

108,833

WESTCOAST GAS SERVICES INC. - N'WESTERN PUB. SERV.

(Westcoast Gas)

AVG Daily Quantity

Total Price (Port of Morgan. MT)

0.9

\begin{tabular}{r}
0.0 \\
0.00 \\
\hline 0.00 \\
0.00
\end{tabular}

0.0
0.00
0.00
0.00

0.0

$\underline{0.00}$

0.0

$\frac{0.00}{0.00}$

Commodity Compone

Reservation Fee Component

0.00

0.00

0.00
0.00

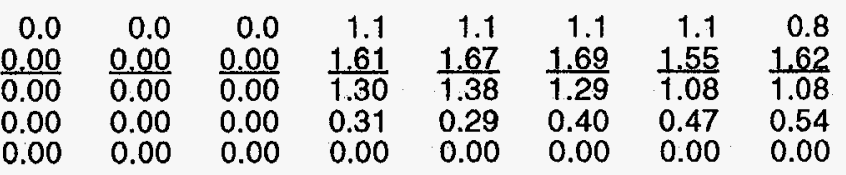

87,992

87,992

241,295

241,295

549,029

549,029

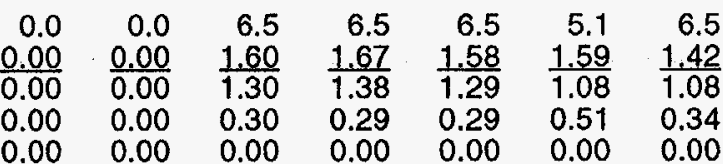

0.34
0.00

$\begin{array}{rrrrrrrr}0.0 & 0.0 & 0.0 & 0.9 & 0.9 & 0.9 & 0.9 & 0.9 \\ \frac{0.00}{0.00} & \frac{0.00}{0.00} & \frac{0.00}{0.00} & \frac{1.90}{1.42} & \frac{1.97}{1.51} & \frac{1.64}{1.42} & \frac{1.44}{1.19} & \frac{1.41}{1.19} \\ 0.00 & 0.00 & 0.00 & 0.48 & 0.46 & 0.22 & 0.25 & 0.22 \\ 0.00 & 0.00 & 0.00 & 0.00 & 0.00 & 0.00 & 0.00 & 0.00\end{array}$

85,419

85,419 
VOLUME AND PRICE REPORT

Long Term Imports

Volumes (MMct/d) \& Prices (\$MMBTU) of Natural

Gas Imported During the Past 12 Months 04/01/94 - 03/31/95

Long-Term importer

$\begin{array}{lllllllllllll}\text { Auth. } & 1994 & 1994 & 1994 & 1994 & 1994 & 1994 & 1994 & 1994 & 1994 & 1995 & 1995 & 1995 \\ \text { Vols. } & \text { Apr. } & \text { May. } & \text { Jun. } & \text { Jul. } & \text { Aug. } & \text { Sep. } & \text { Oct. } & \text { Nov. } & \text { Dec. } & \text { Jan. } & \text { Feb. } & \text { Mar. }\end{array}$

WESTCOAST GAS SERVICES INC. - WISCONSIN GAS CO.

(Westcoast Gas)

AVG Daily Quantity

Total Price (Port of Morgan. MT)

Commodity Component

Demand Component

Reservation Fee Component
2.4

$$
\begin{array}{r}
0.0 \\
0.00 \\
0.00 \\
0.00 \\
0.00
\end{array}
$$

\begin{tabular}{r}
0.0 \\
0.00 \\
\hline 0.00 \\
0.00 \\
0.00
\end{tabular}

0.0
0.00
0.00
0.00
0.00

0.0
0.00
0.00
0.00
0.00

\begin{tabular}{r}
0.0 \\
0.00 \\
\hline 0.00 \\
0.00 \\
0.00
\end{tabular}

0.0
0.00
0.00

$$
0.00
$$

25.0

$$
\frac{15}{1.2}
$$

15.3
2.29
1.70
0.59

11.2
2.33
1.75
0.58
0.00

15.1
2.05
1.44
0.61
0.00

$\begin{array}{lll}0.00 & 0.00 & 0.00\end{array}$

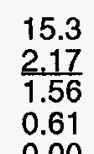

14.

$\frac{2.08}{1.48}$

2.08
0.68
0.00

0.00

3.0

$\begin{array}{rrr}3.0 & 3.0 & 3.0 \\ 2.09 & 2.12 & \frac{1.90}{1.57} \\ \frac{1.61}{1.37} \\ 0.48 & 0.47 & 0.50\end{array}$

0.04

0.04

0.03

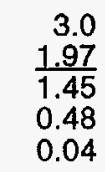

3.0
$\frac{1.85}{1.33}$
0.49

1.33
0.49

0.49
0.03

7.4

\begin{tabular}{lrr}
7.3 & 7.4 & 7.4 \\
2.11 & 2.14 & $\frac{1.95}{1.45}$ \\
\hline 1.63 & 1.67 & 1.45 \\
0.43 & 0.42 & 0.46 \\
0.05 & 0.05 & 0.04
\end{tabular}

7.3
2.01
1.52
0.44
0.05

7.3
1.90
1.43

$\frac{1.90}{1.43}$
0.43

0.43
0.04

$\frac{1.71}{1.21}$

1.21
0.46
0.04

$\frac{1.62}{1.15}$

1.15
0.44
0.03

$\begin{array}{ll}0.03 & 0.05\end{array}$

6.6

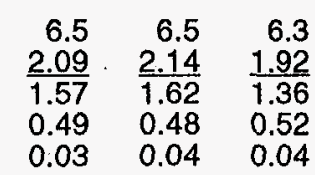

\begin{tabular}{r}
6.4 \\
1.99 \\
\hline 1.46 \\
0.49 \\
0.04
\end{tabular}

6.4
$\frac{1.86}{1.33}$
0.50
0.03

\begin{tabular}{rr}
6.4 & 6.4 \\
86 & 1.63 \\
\hline .33 & 1.08 \\
.50 & 0.52 \\
.03 & 0.03
\end{tabular}

\begin{tabular}{r}
6.5 \\
1.54 \\
\hline 1.01 \\
0.50 \\
0.03
\end{tabular}

6.5
1.79
0.51
0.02

$1 Q 95$

TOTAL MCf

184,022

$\begin{array}{r}2.4 \\ 1.51 \\ \hline 1.16\end{array}$

1.16

.35

Reservation Fee Component

(1) Volumes were resold to Northern Natural. On September 26, 1994, DOE/FE Order 637-C terminated this authorization, effective November 1. 


\section{Long-Term Importer}

WISCONSIN GAS COMPANY

(ProGas Limited (II))

AVG Daily Quantity

Total Price (Noyes, Minnesota)

Commodity Component

Demand Component

Reservation Fee Component

\section{WISCONSIN GAS COMPANY}

(WGM Ltd.)

AVG Daily Quantity

Total Price (Noyes. Minnesota)

Commodity Component

Demand Component

Reservation Fee Component

\section{WISCONSIN GAS COMPANY}

(WGM Ltd.)

AVG Daily Quantity

Total Price (Noyes. Minnesota)

Commodity Component

Demand Component

Reservation Fee Component

WISCONSIN NATURAL. GAS COMPANY

(Progas Limited)

AVG Daily Quantity

Total Price (Noves, Minnesota)

Commodity Component

Demand Component

Reservation Fee Component

\section{WISCONSIN NATURAL GAS COMPANY} (WGM Ltd.)

AVG Daily Quantity

Total Price (Noyes, Minnesota)

modity Component

Demand Component

Reservation Fee Component

\section{VOLUME AND PRICE REPORT}

\section{Long Term Imports}

Volumes (MMcf/d) \& Prices (\$MMBTU) of Natural Gas Imported During the Past 12 Months 04/01/94 - 03/31/95

Auth.
Vols.

Apr. Mav Jun

28.5

\begin{tabular}{|c|c|c|c|c|c|c|c|c|c|c|c|}
\hline $\begin{array}{l}.8 .4 \\
.09 \\
.57 \\
.48\end{array}$ & $\begin{array}{l}28.5 \\
2.13 \\
1.62 \\
0.47 \\
0.04\end{array}$ & $\begin{array}{l}28.4 \\
1.90 \\
1.36 \\
0.51 \\
0.03\end{array}$ & $\begin{array}{l}28.3 \\
1.98 \\
1.46 \\
0.48 \\
0.04\end{array}$ & $\begin{array}{l}28.2 \\
1.85 \\
1.33 \\
0.49 \\
0.03\end{array}$ & $\begin{array}{l}28 . \\
1.6 \\
1.0 \\
0.5 \\
0.0\end{array}$ & $\begin{array}{l}28.3 \\
1.53 \\
1.01 \\
0.49 \\
0.03\end{array}$ & & & & & $\begin{array}{l}28.5 \\
1.51 \\
1.01 \\
0.49 \\
0.01\end{array}$ \\
\hline
\end{tabular}

$\begin{array}{llll}88.6 & 88.9 & 88.8 & 88 \\ 2.10 & 2.13 & 1.94 & 2 \\ 1.62 & 1.66 & 1.44 & 1.5 \\ 0.43 & 0.42 & 0.46 & 0.4 \\ 0.05 & 0.05 & 0.04 & 0.05\end{array}$

1.9

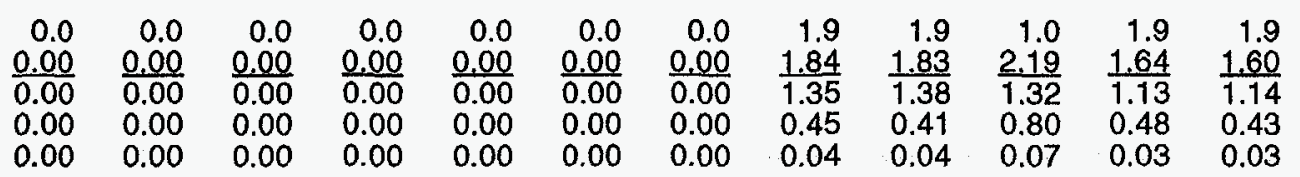

10.7

$\begin{array}{llllllllllll}10.7 & 10.3 & 10.7 & 10.7 & 10.7 & 10.2 & 10.2 & 10.5 & 10.6 & 10.7 & 10.8 & 10.8 \\ \frac{2.09}{1.57} & \frac{2.15}{1.62} & \frac{1.89}{1.36} & \frac{1.98}{1.46} & \frac{1.85}{1.33} & \frac{1.64}{1.08} & \frac{1.55}{1.01} & \frac{1.80}{1.26} & \frac{1.82}{1.30} & \frac{1.76}{1.22} & \frac{1.59}{1.00} & \frac{1.55}{1.01} \\ 0.48 & 0.49 & 0.50 & 0.48 & 0.49 & 0.53 & 0.51 & 0.51 & 0.49 & 0.52 & 0.57 & 0.52 \\ 0.04 & 0.04 & 0.03 & 0.04 & 0.03 & 0.03 & 0.03 & 0.03 & 0.03 & 0.02 & 0.02 & 0.02\end{array}$

26.5

\begin{tabular}{ll}
26.3 & 26.6 \\
2.10 & 2.13 \\
\hline 1.62 & 1.66 \\
0.43 & 0.42 \\
0.05 & 0.05
\end{tabular}

$\begin{array}{ll}0.05 & 0.05\end{array}$

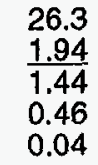

$\frac{2.01}{1.52}$

0.44
0.05

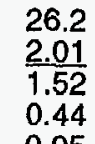

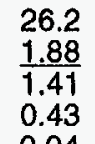

$\frac{1.88}{1.41}$
0.43

0.04

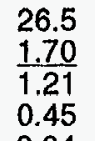

$\frac{1.70}{1.21}$

0.04

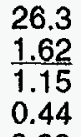

$\frac{1.62}{1.15}$

0.03

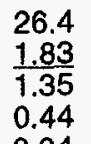

1.83
1.35
0.44

0.04

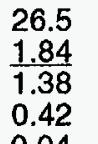

1.84
1.38
0.42
0.04
25.6
$\frac{1.81}{1.32}$
0.45
0.04

\begin{tabular}{r}
20.1 \\
1.80 \\
\hline 1.13 \\
0.63
\end{tabular}

26.3

$\frac{1.60}{1.14}$

0.43

$\begin{array}{ll}0.04 & 0.03\end{array}$
144,072

144,072

966,597

966,597

$2,169,984$

$2,169,984$

(2)
YTD

TOTAL MC

$2,565,676$

$7,403,790$ 
VOLUME AND PRICE REPORT

\section{Long Term Imports}

Volumes (MMct/d) \& Prices (\$MMBTU) of Natural

Gas Imported During the Past 12 Months 04/01/94 - 03/31/95
Long-Term Importer

WISCONSIN POWER \& LIGHT COMPANY (ProGas Limited)

AVG Daily Quantity

Total Price (Noyes, Minnesota)

Commodity Component

Demand Component

Reservation Fee Component

WISCONSIN POWER \& LIGHT COMPANY

(WGM Ltd.)

AVG Daily Quantity

Total Price (Noyes. Minnesota)

Commodity Component

Demand Component

Reservation Fee Component

WISCONSIN POWER \& LIGHT COMPANY

(WGM Ltd.)

AVG Daily Quantity

Total Price (Noyes, Minnesota)

Commodity Componen

Demand Component

Reservation Fee Component

WISCONSIN PUBLIC SERVICE CORPORATION

(ProGas Limited)

AVG Daily Quantity

Total Price (Noyes. Minnesota)

Commodity Component

Demand Component

Reservation Fee Component

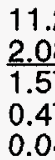

\begin{tabular}{l}
11.2 \\
2.08 \\
\hline 1.57 \\
0.47
\end{tabular}

11.0
2.13
1.62
0.47

$\frac{1.89}{1.36}$

0.50

1994
Jul.

1994

1994

1994

$1994 \quad 1994 \quad 1995$

Apr. May. Jun

.

$\begin{array}{rrr}3.4 & 3.4 & 3.4 \\ 2.05 & 2.09 & 1.8 \\ 1.57 & 1.62 & 1.3 \\ 0.48 & 0.47 & 0.5 \\ 0.00 & 0.00 & 0.00\end{array}$

\begin{tabular}{rr}
3.4 & 3.4 \\
1.94 & $\frac{1.82}{1.33}$ \\
\hline 1.46 & 0.49 \\
0.48 & 0.49 \\
0.00 & 0.00
\end{tabular}

\begin{tabular}{r}
3.4 \\
1.61 \\
\hline 1.10 \\
0.51 \\
0.00
\end{tabular}

3.4
1.51
1.02
0.49
0.00

\begin{tabular}{r}
3.4 \\
1.76 \\
\hline 1.26 \\
0.50 \\
0.00
\end{tabular}

3.4
1.80
1.30
0.50

4.3

$\begin{array}{rrr}4.2 & 4.1 & 4.2 \\ 2.12 & 2.14 & 1.90 \\ 1.52 & 1.55 & 1.28 \\ 0.60 & 0.59 & 0.62\end{array}$

4.2
$\frac{2.10}{1.36}$
0.74

4.2
1.86
1.27
0.59

4.2
1.70
1.09
0.61
0.00

$\begin{array}{r}4.2 \\ +57 \\ \hline\end{array}$

$\frac{1.57}{0.98}$

0.74
0.00

$\begin{array}{ll}0.00 & 0.00\end{array}$

8.4

\begin{tabular}{rrr}
8.2 & 8.2 & 8.2 \\
2.10 & 2.14 & 1.94 \\
\hline 1.62 & 1.66 & 1.44 \\
0.48 & 0.48 & 0.50
\end{tabular}

8.2
$\frac{1.96}{1.52}$

0.44

\begin{tabular}{r}
8.2 \\
1.85 \\
\hline 1.41 \\
0.44
\end{tabular}

$\begin{array}{ll}8.2 & 8.2 \\ 1 & 2.08 \\ 4 & 0.62 \\ 0 & 0.0\end{array}$

\begin{tabular}{rr}
8.2 & 8.2 \\
08 & 1.63 \\
\hline 62 & 1.15 \\
.46 & 0.48
\end{tabular}

\begin{tabular}{rr}
8.2 & 8.3 \\
1.63 & 1.82 \\
\hline 15 & 1.35
\end{tabular}

0.48
0.00

1.82
1.35
0.47

0.47
0.00

\begin{tabular}{r}
8.4 \\
1.84 \\
\hline 1.38 \\
0.46
\end{tabular}

0.46
0.00

$0.00 \quad 0.00$

1995

Feb.

Mar.

$3.4 \quad 3.4$

$4 \quad 1.58 \quad 1.54$

.54

$0.00 \quad 0.00$

WISCONSIN PUBLIC SERVICE CORPORATION

(ProGas Limited)

AVG Daily Quantity

Total Price (Noyes, Minnesota)

8.0

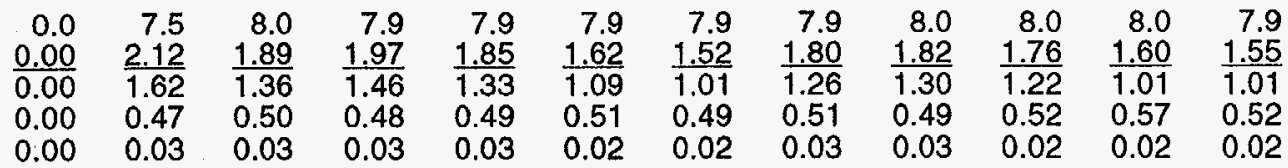

\begin{tabular}{r}
11.1 \\
1.98 \\
\hline 1.46 \\
0.48 \\
0.04 \\
\\
7.9 \\
1.97 \\
\hline 1.46 \\
0.48 \\
0.03
\end{tabular}

11.0

11.1

11.0

$11.0 \quad 11.1$

$\frac{1.84}{1.33} \quad \frac{1.62}{1.08}$

1.08
0.51

1.53
1.01
0.49

1.01
0.49
0.03

$\frac{1.80}{1.26}$

$\frac{1.82}{1.30}$

0.03

0.03

0.03

0.03

\begin{tabular}{l}
11.1 \\
1.77 \\
\hline 1.22 \\
0.52 \\
0.03
\end{tabular}

$11.1 \quad 11.1$

$\frac{1.61}{1.01} \frac{1.56}{1.01}$

$0.57 \quad 0.52$

Commodity Component

Demand Component

Reservation Fee Component

$\begin{array}{rrrrrrrr}7.9 & 7.9 & 7.9 & 7.9 & 8.0 & 8.0 & 8.0 & 7.9 \\ \frac{1.85}{1.33} & \frac{1.62}{1.09} & \frac{1.52}{1.01} & \frac{1.80}{1.26} & \frac{1.82}{1.30} & \frac{1.76}{1.22} & \frac{1.60}{1.01} & \frac{1.55}{1.01} \\ 0.49 & 0.51 & 0.49 & 0.51 & 0.49 & 0.52 & 0.57 & 0.52 \\ 0.03 & 0.02 & 0.02 & 0.03 & 0.03 & 0.02 & 0.02 & 0.02\end{array}$

TOTAL

305,808

YTD TOTAL MCf

305,808

379,444

379,444

751,241

751,241

$1,307,123$

$1,307,123$

717,900

717,900 


\section{VOLUME AND PRICE REPORT}

\section{Long Term Imports}

Volumes (MMct/d) \& Prices ( $\$ M M M B T U$ ) of Natural Gas Imported During the Past 12 Months 04/01/94 - 03/31/95
Long-Term Importer

WISCONSIN PUBLIC SERVICE CORPORATION (WGM Ltd.)

AVG Daily Quantity

Total Price (Noves. Minnesota)

Commodity Component

Demand Component

Peservation Fee Component

YANKEE GAS SERVICES CO.

(Alberta NE Gas (AEC Oil \& Gas Company))

AVG Daily Quantity

Total Price (Waddington, NY)

Commodity Component

Demand Component

Reservation Fee Component

YANKEE GAS SERVICES CO.

(Alberta Northeast Gas (Atcor))

AVG Daily Quantity

Total Price (Waddington. NY)

Commodity Component

Demand Component

Reservation Fee Component

YANKEE GAS SERVICES CO

(Alberta Northeast Gas (ProGas))

AVG Daily Quantity

Total Price (Waddington, NY)

Commodity Component

Demand Component

Reservation Fee Component

YANKEE GAS SERVICES CO

(Alberta Northeast Gas (WGM Ltd. 1))

AVG Daily Quantity

Total Price (Waddington, NY)

Commodity Component

Demand Component

Reservation Fee Component
Àuth.

$\begin{array}{llllll}1994 & 1994 & 1994 & 1994 & 1994 & 1994\end{array}$

Apr. May Jun

27.4

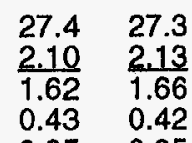

$\begin{array}{ll}0.05 & 0.05\end{array}$

3.9

$\begin{array}{lr}3.9 & 3.9 \\ \frac{2.17}{1.31} & 2.24 \\ 0.86 & 1.39 \\ 0.00 & 0.85\end{array}$

5.2

$\begin{array}{rr}5.2 & 5.2 \\ \frac{2.26}{1.39} & \frac{2.1}{1.29} \\ 0.87 & 0.87 \\ 0.00 & 0.00\end{array}$

9.9

\begin{tabular}{rr}
9.9 & 9.9 \\
2.24 & 2.17 \\
\hline 1.32 & 1.26 \\
0.92 & 0.91 \\
0.00 & 0.00
\end{tabular}

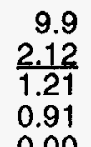

9.8
2.30
1.35
0.95
0.00
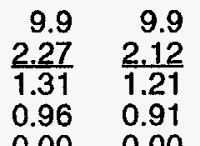

9.9
1.96
0.99
0.97
0.00

9.9
2.36
1.39
0.97
0.00

9.9
2.42
1.44
0.98

9.9
2.38
1.38
1.00
0.00

28.7

$\begin{array}{lllll}28.7 & 28.7 & 28.7 & 28.7 & 28.7 \\ 2.24 & 2.17 & 2.12 & \frac{2.25}{1.35} & \frac{2.23}{1.31} \\ 1.37 & 1.30 & \frac{1.25}{1.25} & 0.90 & 0.92 \\ 0.87 & 0.87 & 0.87 & 0.00 & 0.00\end{array}$

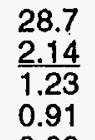

28.7
$\frac{2.14}{1.23}$
0.91
0.00

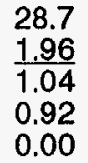

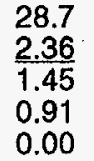

28.7
2.36
1.45
0.91
0.00

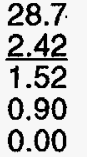

28.7
2.38
1.47
0.91
0.00
9.9
2.32
1.34
0.98

9.9
2.26
1.27
0.99
0.00 \begin{tabular}{l}
28.7 \\
2.31 \\
\hline 1.42 \\
0.89
\end{tabular}

28.7
2.26
1.36
0.90 $\begin{array}{lll}00 & 0.00 & 0.00\end{array}$

$1 Q 95$ TOTAL Mc

$2,457,169$

$2,457,169$

$\frac{1.60}{1.14}$

0.43

0.03

3.9

$\frac{2.26}{1.36}$

0.90

0.00

469,980

469,980

891,000

891,000

$2,583,000$

$2,583,000$

YTD

OTAL Mcf

353,520

353,520

.

409 


\section{VOLUME AND PRICE REPORT}

\section{Long Term Imports}

Volumes (MMct/d) \& Prices (\$MMBTU) of Natural

Gas Imported During the Past 12 Months 04/01/94 - 03/31/95

Long-Term Importer

YANKEE GAS SERVICES CO

(Alberta Northeast Gas (WGM Ltd. 2))

AVG Daily Quantity

Total Price (Waddington. NY)

Commodity Component

Demand Component

Reservation Fee Component

Total Mef
Auth.
Vols.

1994

\begin{tabular}{lll}
1994 & 1994 & 1994 \\
Apr. May, & Jun \\
\hline
\end{tabular}

1994

1994

1994

1994

1994.

$\begin{array}{ll}1994 & 1995 \\ \text { Dec. } & \text { Jan. }\end{array}$

1995

Feb.

1995

Mar.

11.3

$\begin{array}{llll}11.3 & 11.3 & 11.3 & 11 \\ 2.24 & 2.17 & 2.12 & 2 \\ \frac{2.37}{1.30} & 1.25 & 1.30 \\ 0.87 & 0.87 & 0.87 & 0.87 \\ 0.00 & 0.00 & 0.00 & 0.00\end{array}$

$\begin{array}{ll}11.3 & 11.3 \\ \frac{2.17}{1.30} & 2 \\ 0.87 & 0.32 \\ 0.00 & 0.00\end{array}$

11.3
2.22
1.32
0.90
0.00

11.3
2.12
1.25
0.87

11.3
1.96

.3
.04
.92
.00

11.3
2.36
1.45
0.91
0.00

$\begin{array}{ll}11.3 & 11.3\end{array}$

$\frac{2.42}{1.52}$

11.3
$\frac{2.38}{1.47}$

11.3

$\frac{2.31}{1.42}$

11.3

$\frac{2.26}{1.36}$

$\frac{2.26}{1.36}$

$\begin{array}{lllllll}. .87 & 0.92 & 0.91 & 0.90 & 0.91 & 0.89 & 0.90 \\ 0.00 & 0.00 & 0.00 & 0.00 & 0.00 & 0.00 & 0.00\end{array}$ 


\section{VOLUME AND PRICE REPORT}

\section{Long Term Exports}

Volumes (MMcf/d) \& Prices (\$MMBTU) of Natural

Gas Exported During the Past 12 Months 04/01/94 - 03/31/95

Long-Term Exporter

MARATHON OIL COMPANY

(Tokyo Electric Co./Tokyo Gas Co.)

AVG Daily Quantity

Total Price (Kenai, Alaska)

Commodity Component

Demand Component

Reservation Fee Component

Vols.

Apr

May. Jun

$\begin{array}{lllllllll}1994 & 1994 & 1994 & 1994 & 1994 & 1994 & 1995 & 1995 & 1995\end{array}$

50.4

$\begin{array}{lllllllllllll}36.6 & 37.7 & 58.2 & 53.6 & 51.1 & 58.3 & 52.4 & 54.0 & 72.0 & 53.7 & 56.4 & 54.2 \\ \frac{2.89}{2.89} & \frac{2.92}{2.92} & \frac{2.99}{2.99} & \frac{3.12}{3.12} & \frac{3.26}{3.26} & \frac{3.36}{3.36} & \frac{3.38}{3.38} & \frac{3.34}{3.34} & \frac{3.31}{3.31} & \frac{3.32}{3.32} & \frac{3.34}{3.34} & \frac{3.34}{3.34} \\ 0.00 & 0.00 & 0.00 & 0.00 & 0.00 & 0.00 & 0.00 & 0.00 & 0.00 & 0.00 & 0.00 & 0.00 \\ 0.00 & 0.00 & 0.00 & 0.00 & 0.00 & 0.00 & 0.00 & 0.00 & 0.00 & 0.00 & 0.00 & 0.00\end{array}$

PHILLIPS ALASKA NATURAL GAS CORPORATION • (Tokyo Electric Co./Tokyo Gas Co.)

AVG Daily Quantity

136.4

2.89

$\frac{2.89}{2.89}$

\begin{tabular}{lr}
81.4 & 12 \\
2.92 & 2 \\
\hline 2.92 & 2 \\
0.00 & 0.0
\end{tabular}

$\begin{array}{lll}0.00 & 0.00 & 0\end{array}$

Demand Component

Reservation Fee Component

Total Mof 


\section{SHORT-TERM BLANKET IMPORTS}

Year \&

Month

Active

Importers
Est. Volumes

(MMCF)
Weighted Avg.

Price (\$/MMBTU)

1993

January

February

March

April

May

June

July

August

September

October

November

December

1994

January

February

March

April

May

June

July

August

September

October

November

December

1995

January

February

March
64

70

71

63

62

59

66

67

67

68

87

95

100

102

89

92

91

90

94

101

95

98

89

94

97

100

94
59879

62911

70845

61000

53853

53821

63432

70638

62626

61610

89945

102632

114799

89789

104609

93536

88377

87001

100339

105103

100031

105103

106733

113499
1.84

1.58

1.67

1.71

1.84

1.62

1.58

1.62

1.80

1.67

1.92

2.13

1.97

2.02

1.95

1.69

1.66

1.49

1.49

1.45

1.32

1.22

1.40

1.42

131167

1.29

1.15

1.11 
SHORT-TERM IMPORTERS

Estimated Volumes (MMCF)

1 SOURCE ENERGY SERVICES COMPANY ACCESS ENERGY COFIPORATION

AIG TRADING CORPOFIATION

ALTRESCO PITTSFIELD, L.P.

AMERADA HESS CORFORATION

AMERICAN HUNTER EXPLORATION LTD.

AMOCO CANADA MARIKETING CORPORATION

AMOCO ENERGY TRADING CORPORATION

ANADARKO TRADING COMPANY

ANR GAS SUPPLY COMPANY

ANR PIPELINE COMPANY

ARCO PRODUCTS COMPANY

ASSOCIATED GAS SEFIVICES, INC.

BAY STATE GAS COMPANY

BC GAS UTILITY LTD.

BROAD STREET OIL \& GAS COMPANY

BRYMORE ENERGY INC.

CABOT OIL \& GAS TRADING CORPORATION

CANADA IMPERIAL OIL. LIMITED

CANADIAN HYDROCAFBBONS MARKETING (U.S.) INC

CANADIANOXY MARKETTING INC.

CANSTATES GAS MARKETING (U.S.) Ltd.

CANWEST GAS SUPPL.Y U.S.A., INC.

CASCADE NATURAL GAS CORPORATION

CATEX VITOL GAS, INC.

CHEVRON NATURAL GAS SERVICES INC.

CHEVRON U.S.A. INC.

CIBOLA CORPORATION

CMEX ENERGY, INC.

CNG PRODUCING COMPANY

COASTAL GAS MARKETING COMPANY

COENERGY TRADING COMPANY

CONSUMERS POWER COMPANY

CONTINENTAL ENERGY MARKETING LTD.

CONWEST EXPLORATION COMPANY LIMITED

CRESTAR ENERGY MARKETING CORPORATION

CU ENERGY MARKETING, INC.

DARTMOUTH POWER ASSOCIATES L.P.

DEKALB ENERGY COMPANY

DGS TRADING INC.

DIRECT ENERGY MARKETING LIMITED

EASTERN ENERGY MARKETING, INC.

ENRON GAS MARKETING, INC.

ENRON GAS SERVICES CANADA CORPORATION EQUITABLE RESOURCES MARKETING COMPANY GAZ METROPOLITAIN AND COMPANY, L.P.

GREAT FALLS GAS COMPANY
1994

Jan.-Mar.

1994

Apr-Jun

1994

33

119

7206

0

0

2093

5073

648

310

464

3480

741

14466

555

0

213

0

5686

799

1563

0

15694

4029

0

7145

0

23

560

604

2942

269

0

947

0

322

768

337

1070

545

1282

0

8020

46

0

0

0

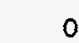

67

448

0

0

89

3859

1085

574

633

3518

697

10972

263

0

383

0

450

197

1096

0

1239

16215

744

0

7590

0

0

0

421

1131

0

0

261

r

700

1226

179

995

1001

562

0
7034

0

0

0

45
Jul.-Sep

1994

Oct.-Dec

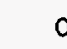

69

1143

2804

0

0

3570

0

0

953

3524

945

9228

0

0

377

159

415

121

364

0

1582

12974

2098

82

0

8472

0

0

0
3141

3141

4342

851

553

688

1457

135

1167

377

215

0

14600

0

0

0

25
1995

Jan.-Mar.

0

0

7782

2754

1693

0

5993

805

0

0

1740

1153

7699

555

76

214

0

441

344

0

206

4397

19158

4476

0

0

8921

731

0

0

7974

548

6866

1121

818

606

613

204

930

2585

1544

8317

0

882

1667

452 


\section{SHORT-TERM IMPORTERS}

Estimated Volumes (MMCF)

GREAT PLAINS NATURAL GAS COMPANY

HOWARD ENERGY COMPANY, INC.

HUSKY GAS MARKETING, INC.

IGI RESOURCES INC.

INDECK ENERGY SERVICES, INC.

INLAND PACIFIC ENERGY SERVICES CORP.

INLAND PACIFIC ENERGY SERVICES LTD.

INTALCO ALUMINUM CORPORATION

INTERENERGY CORPORATION

INTERSTATE POWER COMPANY

INVERNESS RESOURCES INC.

IOWA ELECTRIC LIGHT \& POWER COMPANY

IROQUOIS ENERGY MANAGEMENT, INC.

JMC FUEL SERVICES, INC.

JONAN GAS MARKETING

KCS ENERGY MARKETING, INC.

KIMBALL ENERGY CORPORATION

KOCH GAS SERVICES COMPANY

LOUIS DREYFUS ENERGY CORPORATION

MADISON GAS \& ELECTRIC COMPANY

MASSPOWER

METROPOLITAN UTILITIES DISTRICT

MIDCON GAS SERVICES CORPORATION

MIDWEST GAS COMPANY

MINNEGASCO

MOBIL NATURAL GAS INC.

MOCK RESOURCES, INC.

MURPHY GAS GATHERING

NATIONAL FUEL GAS DISTRIBUTION

NATIONAL STEEL CORPORATION

NATURAL GAS CLEARINGHOUSE INC.

NEW ENGLAND POWER COMPANY

NORCEN MARKETING INC.

NORSTAR ENERGY, L.P.

NORTH AMERICAN RESOURCES COMPANY

NORTH CANADIAN MARKETING CORPORATION

NORTHERN ILLINOIS GAS COMPANY

NORTHERN MINNESOTA UTILITIES

NORTHERN STATES POWER COMPANY (MINNESOTA)

NORTHERN STATES POWER COMPANY (WISCONSIN)

NORTHERN UTILITIES, INC.

NORTHRIDGE GAS MARKETING, INC.

NORTHWESTERN PUBLIC SERVICE COMPANY

NOVAGAS CLEARINGHOUSE LTD.

NUMAC ENERGY (U.S.) INC.

O\&R ENERGY, INC.

OCEAN STATE POWER

\begin{tabular}{|c|c|}
\hline $\begin{array}{c}1994 \\
\text { Jan.-Mar. }\end{array}$ & $\begin{array}{r}1994 \\
\text { Apr-Ju } \\
\end{array}$ \\
\hline 53 & 51 \\
\hline 117 & 0 \\
\hline 9891 & 10257 \\
\hline 3822 & 3688 \\
\hline 357 & 321 \\
\hline 734 & 636 \\
\hline 1947 & 625 \\
\hline 274 & 239 \\
\hline 618 & 318 \\
\hline 66 & 56 \\
\hline 343 & 344 \\
\hline 241 & 154 \\
\hline 5 & 65 \\
\hline 0 & 0 \\
\hline 58 & 142 \\
\hline 483 & 643 \\
\hline 1175 & 918 \\
\hline 0 & 0 \\
\hline 358 & 0 \\
\hline 88 & 88 \\
\hline 0 & 0 \\
\hline 495 & 457 \\
\hline 0 & 0 \\
\hline 458 & 580 \\
\hline 2372 & 5480 \\
\hline 9435 & 8893 \\
\hline 105 & 170 \\
\hline 293 & 296 \\
\hline 0 & 0 \\
\hline 1618 & 1668 \\
\hline 249 & 0 \\
\hline 246 & 0 \\
\hline 1089 & 0 \\
\hline 0 & 0 \\
\hline 177 & 854 \\
\hline 9615 & 9147 \\
\hline 609 & 226 \\
\hline 1072 & 395 \\
\hline 2078 & 2010 \\
\hline 318 & 277 \\
\hline 86 & 59 \\
\hline 2040 & 1896 \\
\hline 83 & 85 \\
\hline 150 & 152 \\
\hline 0 & 0 \\
\hline 0 & 25 \\
\hline 0 & 0 \\
\hline
\end{tabular}

1994

Jul. -Sep

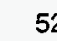

136

10904

4037

459

698

455

211

409

75

336

247

67

0

313

374

727

0

0

88

628

0

358

2138

9933

953

302

0

1586

0

0

0

0

492

11339

530

343

1781

333

0

2090

84

154

0

106

106
1994

Oct.-Dec.

1995 Jan.-Mar.

18

19

10123

8856

441

574

2426

163

234

33

347

85

606

0

229

1044

731

952

0

31

301

179

1996

202

798

8798

0

299

0

1736

0

0

0

361

9210

213

782

379

113

58

2932

29

154

1188

1209

0
0

0
8622

11357

462

473

4095

122

348

0

339

0

937

10

126

620

898

3229

364

0

317

0

2712

0

0

15621

0

293

266

2189

0

0

4110

991

343

12900

0

497

0

0

260

3858

0

124

1817

0

50 
OCEAN STATE POWER \|

ORYX GAS MARKETING, L.P.

PACIFIC GAS TRANSMISSION COMPANY

PAN NATIONAL GAS SALES, INC. (1)

PAN-ALBERTA GAS (U.S.), INC.

PANCANADIAN PETROLEUM COMPANY

PARAMOUNT RESOUFICES U.S., INC.

PAWTUCKET POWER ASSOCIATES L.P.

PEOPLES NATURAL GAS COMPANY

PETRO-CANADA HYDFOCARBONS, INC.

PG\&E ELECTRIC SUPF'LY BUSINESS UNIT

PG\&E GAS SUPPLY BUSINESS UNIT

PHIBRO OIL \& GAS, INC.

POCO PETROLEUM, INC.

POWER CITY PARTNERS, L.P.

PROGAS U.S.A., INC.

RENAISSANCE ENERGY (U.S.), INC.

SALMON RESOURCES LTD.

SAN DIEGO GAS \& ELECTRIC

SELKIRK COGEN PARTNERS, L,P.

SIERRA PACIFIC POWER COMPANY

SOUTHEASTERN MICHIGAN GAS COMPANY

SOUTHERN CALIFORNIA EDISON COMPANY

ST. CLAIR PIPELINES LITD.

STAMPGAS (U.S.) INC.

SUNCOR INC.

SUNRISE ENERGY COMPANY

TALISMAN MARKETING (U.S.)

TECO GAS MARKETING COMPANY

TENASKA GAS COMPANY

TENASKA MARKETING VENTURES

TENNECO GAS MARKETING COMPANY

TEXACO GAS MARKETING INC.

TEXAS-OHIO GAS, INC.

THE MONTANA POWER COMPANY

TRANSCO ENERGY MARKETING COMPANY

TRISTAR GAS MARKETING COMPANY

UNION GAS LIMITED

UNION PACIFIC FUELS, INC.

UNIVERSAL RESOURCES CORPORATION

UTILICORP UNITED

VECTOR ENERGY (U.S.A.) INC.

VERMONT GAS SYSTEEMS, INC.

WASCANA ENERGY MARKETING (U.S.) INC.

WASHINGTON ENERGY GAS MARKETING COMPANY

WASHINGTON NATURAL GAS COMPANY

WASHINGTON WATER: POWER COMPANY
1994

Jan.-Mar.

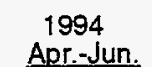

0

0

0
133

6563

5410

1161

103

813

6301

326

53496

2249

5500

1096

4043

3215

639

0

2671

18

4605

0

0
7987

993

0

85

130

0
676

676
323

0

309

3376

266

400

0

0

0
1526

296

6036

0

568

0
Apr-Jun.

1994

Jul.-Sep

0

0
140

0

1513

6309

5508

1723

342

1223

5090

921

52063

433

5403

0

10450

4108

3061

623

0

2691

0

4988

0

0
8863

1212

0

209

90

208

0

0

113

0

3804

0

0

970

0

1542

0

4943

1902

449

64

Co

0

961

9080

6295

1778

30

1295

4904

473

50665

2050

5984

0

9786

6186

3880

733

680

2724

0

5080

0

9620

1479

11

11

52

283

2028

0

408

0

4413

335

0

0
1110

1110

1509

14

3461

4481

453

0
1994
Oct.-Dec.

1995

Jan.-Mar. o
28

0

1156

9625

12942

2056

3

0
7115

7115

902

52470

6220

7183

44

16308

10348

3008

1064

72

1334

0

0

1657

22

9310

609

0

199

0

622

0

285

613

3285

56

0

950

0

593

1504

205

6091

831

673

(1) These figures repressent sales for resale rather than imports. Actual imports are as follows: 1st Qtr 94 (173); 2nd Qtr 94 (2794); 3rd Qtr 94 (47); 4th Qtr 94 (0); 1st Otr 95 (2573). 
SHORT-TERM IMPORTERS

Estimated Volumes (MMCF)

WESTCOAST GAS SERVICES (U.S.A.), INC.

1994

Jan-Mar.

WESTCOAST GAS SERVICES INC.

WESTERN GAS MARKETING INC.

WESTERN GAS RESOURCES, INC.

WISCONSIN GAS COMPANY

WISCONSIN POWER \& LUGT COMPANY

WISCONSIN PUBLIC SERVICE CORPORATION

Totals

580
1685
22248
99
11316
104
718

$\underline{309196}$

$$
\begin{gathered}
1994 \\
\text { Apr.-Jun. }
\end{gathered}
$$

1428

1830

18646

733

175

0

255

268915
1994

Jul.-Sep.

1369

2048

30087

373

177

0

0

305473

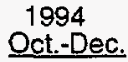

785

3369

25640

372

60

1

0

325336
1995

Jan.-Mar.

47

6107

24572

468

0

0

0

$\underline{383193}$ 
Page -58

PURCHASERS OF SHORT-TERM GAS

Estimated Volumes Imported (MMCF)

1 Source Energy Services Company

AA Productions

AIG Trading Corporation

ANR Gas Supply

ANR Pipeline Company

Access Energy Corporation

Acco World-East

Acco World-West

Alberta Natural Gas

Alberta Northeast Gas Limited

Allied Producers Gas Service, Inc.

Altresco Pittsfield, L.P.

Aluminum Company of America

AmGas Inc.

Amalgamated Sugar Company

Amerada Hess

American Fine Foods

American Home Foods

American Hunter Energy

Amoco Trading

Anadarko Trading Company

Anodizing

Appalachian Gas Sales

Aquila Energy Marketing

Arcadian Corporation

Archer-Daniels-Midland Company

Arco Products Company

Arkla Energy Marketing

Armstrong World Industries, Inc.

Asarco Inc.

Ash Grove Cement

Ash Grove Cement West

1994

Jan-Mar

1994

Apr.-Jun.

1994

Jul.-Sep.

1994

Oct-Dec.

1995

(16

166

59

167

961

634

Jan.-Mar.

Associated Gas Services, Inc.

3480

3480

295

160

0

0

121

2040

115

4502

3524

3518

0

0

2465

1740

8569

3

0

2058

0

0

221

154

9

2037

0

3

4

23

0

0

2804

0

1242

941

2

0

$\begin{array}{rr}1 & 40 \\ 0 & 0\end{array}$

163

344

4931

4434

310

2337

0

411

411
0

0

1460

0

124

28

163

0

5439

Associated Natural Gas

0

Atochem

Ault Naval Base

$B C$ Gas Iric.

BNG, Inc.

Baker Commodities

Baltimore Gas \& Electric

Barretts Minerals

Basic American Foods

Bay State Gas Company

Bethlehem Steel Corporation

Blitz Brewing

Boeing Company

Boise Cascade Corporation

Boston Edison Company

Brammer Manufacturing Company 


\section{PURCHASERS OF SHORT-TERM GAS} Estimated Volumes Imported (MMCF)

Broad Street Oil \& Gas

Brooklyn Interstate Natural Gas

Brooklyn Union Gas

Brymore Energy Inc.

Buffalo Psychiatric

Bullseye Glass

CMS Gas Marketing

CNG Energy Services

CNG Gas Services

CNG Producing Company

CNG Transmission Corporation

Cabot Oil

Califomia Industrial End-users

Califomia Protein Products

CanStates Gas Marketing

Canada Imperial Oil

Canadian Hunter (U.S.)

Canadian Hydrocarbons

CanadianOxy Marketing Inc.

Canton Central School

Canton-Potsdam Hospital

Canwest Gas Supply

Camation Dairies

Cascade Natural Gas

Cascade Steel

Catex Vitol

Cenergy, Inc.

Cenex, Inc.

Centra Gas

Central Hudson Gas \& Electric

Central Hudson Gas \& Electric/CNG Energy

Central Hudson Gas \& Electric/Northeast Utilities

Central Illinois Light Company

Certainteed Corporation

Chautauqua Energy Marketing Inc.

Chevron Chemical

Chevron Natural Gas Services, Inc.

Chevron U.S.A. Inc.

Chooljian Brothers

Cibola Corporation

City of Buckley, OR

City of Ellensburg, WA

City of Enumclaw, WA

City of Greenwood, SC

City of Ogdensburg School District

City of Ogdensburg, NY

City of Palo Alto, CA

City of Pasadena, CA

City of Perham, MN

Clarkson University

\begin{tabular}{|c|c|c|c|c|}
\hline $\begin{array}{c}1994 \\
\text { Jan.-Mar. }\end{array}$ & $\begin{array}{c}1994 \\
\text { Apr--Jun. }\end{array}$ & $\begin{array}{r}1994 \\
\text { Jul.-Sep. } \\
\end{array}$ & $\begin{array}{c}1994 \\
\text { Oct.-Dec. }\end{array}$ & $\begin{array}{c}1985 \\
\text { Jan.-Mar. }\end{array}$ \\
\hline 261 & 22 & 377 & 0 & 333 \\
\hline 228 & 134 & 96 & 297 & 440 \\
\hline 937 & 43 & 246 & 198 & 312 \\
\hline 0 & 0 & 104 & 0 & 0 \\
\hline 0 & 0 & 0 & 86 & 0 \\
\hline 8 & 24 & 0 & 15 & 8 \\
\hline 0 & 0 & 0 & 0 & 181 \\
\hline 0 & 0 & 0 & 0 & 459 \\
\hline 874 & 1142 & 1787 & 968 & 458 \\
\hline 604 & 421 & 0 & 0 & 0 \\
\hline 0 & 0 & 71 & 88 & 0 \\
\hline 0 & 0 & 0 & 0 & 8 \\
\hline 0 & 0 & 0 & 0 & 964 \\
\hline 0 & 0 & 0 & 0 & 90 \\
\hline 146 & 121 & 0 & 493 & 567 \\
\hline 799 & 197 & 121 & 0 & 344 \\
\hline 0 & 0 & 34 & 90 & 0 \\
\hline 68 & 0 & 0 & 0 & 0 \\
\hline 0 & 0 & 0 & 0 & 206 \\
\hline 5 & 5 & 5 & 8 & 4 \\
\hline 5 & 5 & 5 & 5 & 5 \\
\hline 35 & 0 & 0 & 0 & 20 \\
\hline 263 & 228 & 148 & 188 & 24 \\
\hline 5318 & 1330 & 3397 & 4428 & 8396 \\
\hline 80 & 154 & 193 & 128 & 81 \\
\hline 0 & 0 & 229 & 609 & 8 \\
\hline 1493 & 1189 & 2628 & 2088 & 1518 \\
\hline 0 & 10 & 0 & 153 & 62 \\
\hline 0 & 0 & 0 & 0 & 1503 \\
\hline 0 & 764 & 621 & 279 & 70 \\
\hline 0 & 0 & 0 & 473 & 0 \\
\hline 0 & 0 & 0 & 315 & 0 \\
\hline 21 & 0 & 0 & 67 & 0 \\
\hline 326 & 293 & 329 & 302 & 190 \\
\hline 126 & 0 & 102 & 377 & 177 \\
\hline 0 & 0 & 0 & 0 & 920 \\
\hline 7145 & 7945 & 0 & 0 & 0 \\
\hline 89 & 109 & 9255 & 9436 & 11054 \\
\hline 0 & 0 & 14 & 17 & 3 \\
\hline 7883 & 7785 & 8354 & 10530 & 9848 \\
\hline 64 & 0 & 0 & 0 & 0 \\
\hline 128 & 87 & 83 & 125 & 147 \\
\hline 33 & 0 & 0 & 0 & 0 \\
\hline 50 & 0 & 0 & 0 & 0 \\
\hline 9 & 9 & 9 & 9 & 0 \\
\hline 2 & 3 & 3 & 3 & 2 \\
\hline 1088 & 902 & 756 & 1277 & 1086 \\
\hline 0 & 0 & 73 & 0 & 0 \\
\hline 174 & 131 & 142 & 165 & 175 \\
\hline 38 & 38 & 40 & 36 & 33 \\
\hline
\end{tabular}


PURCHASERS OF SHORT-TERM GAS

Estimated Volumes Imported (MMCF)

Clinton - Newbery

Clinton Gas Marketing

CoEnergy Trading Company

Coastal Gas Marketing Company

1994 Jan. Mar.

Cogen Energy Technology

College of Technology

Columbia Aluminum

Columbia Energy Service

Columbia Falls Aluminum

Com Products

Comgas

Commonwealth Gas Company

Connecticut Natural Gas

Connecticut Power \& Light

Conoco Inc.

Consolidated Edison

Consumers Power Company

Continental Baking

Continental Energy Marketing Lid.

Cook Inlet

Cook Inlet/Arco Watson Cogeneraton

Cook Inlet/Mock Resources

Cook Inlet/National \& Redwood Resources

Cook Inlet/National Gas Resources

Cook Inlet/Redwood/Mock \& National Resources

Comell Linen

Crysen Refining

DAl Oildale

DFSC Fort Drum

DGS Trading

Dairyman's Cooperative: Creamery

Darigold Inc.

Darling Delaware

Dartmouth Power Associates

Del Ray Packing

Delmarva Power \& Light Company

Destec Energy

Destec Gas Services

Development Associates

Dexel inc.

Direct Energy Marketing

Direct Gas Marketing

Domtar Gypsum

Dyno Polymers

ESCO Corporation

Eagle Picher

Eastem Energy Marketing

Eastem Shore

Eastex Energy

Eastex Hydrocarbons

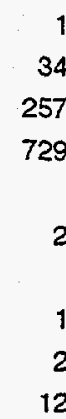

14

7298

0

21

0

19

22

122

0

0

551

0

14

390

359

0

1309

1171

0

0

0

$$
0
$$$$
0
$$$$
6
$$$$
0
$$$$
0
$$

11

1186

0

0

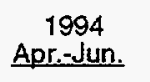

1994
Jul.-Sep.

1994
Oct.-Dec.

1995

Jan.-Mar.
0

229
1730

8041

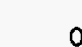

258

939

7757

7214

1730

0

736

1748

0

0

0

0

0

6

0

0

0

737

0

0

0

0

0

251

308

144

176

0

205

0

105

0

49

0

0

0

0

4
26

22

47

0

15

114

9

229

0

0

0
764

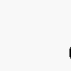

90

1793

8029

18

21

64

0

31

107

50

0
728

306

87

20

9103

5

237

327

604

0

0

1198

0

6

74

0

0

590

292

253

12

50

17

270

254

0

0

61

0

315

0

107

0

48

51

0

0

0

0
28

2101

14248

20

21

72

0

7

78

0

0

0

134

0

16

8527

15

478

1549

0

896

- 551

0

609

6

0

160

0

1

293

328

19

117

6

110

0

0

0

0

67

352

161

6

17

0

91

0

50

0 
PURCHASERS OF SHORT-TERM GAS

Estimated Volumes Imported (MMCF)

El Paso Natural Gas

Elf Atochem

Empire Natural Gas Corporation

Empire State Pipeline

Encogen Northwest, L.P.

Energy Transportation

Enron Capital \& Trade Resources

Enron Gas Marketing

Enron Gas Services

Enron Gas Supply Company

Ensearch Development Corporation

Equitable Resources

Equitable Resources Marketing Company

Evanite Fiber

Evergreen Oil Inc.

Fincrest Schools

Fitzbur

Flanders inn

Flavorland Foods

Fujitsu

G M Hydrocarbons, Ltd.

Gas Company of New Mexico

Gas Energy Development

Gaslantic

Gaylord Container Corporation

General Chemical

1994
Jan.-Mar.

Gilroy Energy Company

Goal Line

Grand Valley Gas Company

Great Falls Gas Company

Great Lakes Gas Transmission Co.

Great Plains Natural Gas Company

Greeley Gas Company

HUB Marketing

HUB Services

Hadson Gas Marketing

Hadson Gas Systems

Hawkeye Manufacturing Company

Hazelton Fuel Management Co.

Heartland Energy

Hercules

Hesse Gas Company

Heuvelton Central School

High Cascade Lumber

Highland Nursing Home

Holman Inc.

Home Oil

Hoosier Magnetics, Inc.
1994
Apr.-Jun.

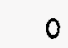

56

47

0

121

15

0

4543

0

0

65

44

0

71

32

0

0

1

0

0

145

23

22

16

984

36

47

295

1994

Jul.-Sep.

1994

Oct.-Dec.

1995

Jan.-Mar.

$\begin{array}{rr}30 & 0 \\ 7 & 0 \\ 54 & 0 \\ 209 & 116\end{array}$

394

0

0

0

15828

2

348

0

1391

2012

535

0

83

29

0

0

1

0

0

147

0

0

0

888

36

68

16

0

0

45

17

566

1024

17

0

0

1514

0

234

0

0
1156

3

0

1

0

25

2076 
Page - 62

PURCHASERS OF SHORT-TERM GAS

Estimated Volumes Imported (MMCF)

Hopewell Cogeneration Facility

Howard Energy Company

Hub Services

Hunt Wesson

Husky Oil

Hytek Finishes

IBP

ICC

IGI Resources

Imperial Oil Resources

Indeck Energy Services of Oswego

Indeck Energy Services of Yerkes

Industrial Energy Applications

Industrial Energy Services Company

Inland Pacific

Inland Steel Company

Intalco Aluminum Corporation

Interenergy Corporation

Intermountain Gas Company

Intermountain Pipeline

Interox

Interstate Gas Marketing

Interstate Power Company

lowa Electric Light \& Power

lowa Illinois Gas \& Electric

Iroquois Energy Management

Iroquois Gas Transmission

J. Aaron Company

J. Makowski

J.R. Simplot Company

JMC Fuel Services

Jackson Prairie Storage

James Hardy

James River Corporation

Joanna A. CHF Company

John Brown Oil \& Gas

Jorgensen Steel

Jorgenson Forge

KCS Energy Management

KN Energy

KTM

Kalama Chemical

Kaltum

Kamine/Besicorp Carthage L.P.

Kamine/Besicorp Natural Dam L.P.

Kamine/Besicorp South Glens Falls

Kamine/Besicorp Syracuse L.P.

Kaztex Energy Managernent

Kimball Energy Corporation

Kimball Trippe Inc.
1994

Jan.-Mar.

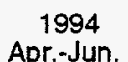

Apr.-Jun.

115

169

122

0

0

0

78

7

8613

0

216

141

109

0

0

362

274

664

672

342

204

13

66

241

0

70

0

1486

4

2472

89

28

117

1399

5

0

0

0

151

0

311

0

159

33

137

11

44

900

10
1994

Jul.-Sep.

449

0

436

0

31

0

93

0

4527

0

207

114

0

157

903

343

239

967

556

446

41

0

56

154

0

67

0

1820

0

1018

$$
0
$$

0

$$
0
$$

1474

5

0

0

0

187

0

258

0

141

164

18

239

0

526

1184

0

446

0

72

0

0

92

4788

0

219

240

0

0

1529

311

211

1190

493

284

176

0

75

247

0

136

56

0

80

1443

5

0

0

1782

5

45

0

0

197

0

141

0

151

138

0

110

33

0

690

0 \begin{tabular}{l}
$\begin{array}{c}1994 \\
\text { Oct.-Dec. }\end{array} \quad$ Jan.-Mar. \\
\hline
\end{tabular}

20

19

$$
\begin{array}{r}
5 \\
293 \\
0 \\
0 \\
0 \\
16 \\
0 \\
0 \\
6334 \\
0 \\
200 \\
261 \\
0 \\
0
\end{array}
$$

16334

1388

372

122

1076

0

0

10

0

0

0

891

465

1661

294

0

1733

188

0

15

1447

0

14

64

0

887

0

393

86

0

0

0

0

0

0

1728 
PURCHASERS OF SHORT-TERM GAS

Estimated Volumes imported (MMCF)

Koch Gas Services Company

Koch Hydrocarbon Company

L.P. McNear Brick Co., Inc.

LG\&E Westmoreland

Lake Cogen Ltd.

Lamb Weston

Legacy Health System

Lisbon Central School

Lockport Energy

Long Island Lighting Company

Louis Dreyfus Energy Corporation

Louisiana Pacific

$M$ \& B Industrial Gas Development

MG Natural Gas Corporation

Madison Gas \& Electric

Madrid-Waddington School

Masonite

Massena Central School

Massena Hospitality Enterprises

Massena Memorial Hospital

Masspower

McCall Oil

Medina Power Company

Meridian Marketing

Metropolitan Utilities District

MichCon Storage

MichCon Trading

Michigan Consolidated Gas Company

Michigan Gas Company

Michigan Gas Utilities

Michigan Gas Ventures

Mid American Natural Resources

Midcon Gas Services Corporation

Midcon Marketing Corporation

Midland Cogeneration Venture

Midland Marketing

Midwest Gas Company

Migas Exchange

Minnegasco

Mobil Gas Marketing Ine.

Mobil Natural Gas Inc.

Mock Resources

Mohawk Correctional

Montana Resources

Montana State

Montana-Dakota Utilities Co.

Morgan Stanley

Mountain Fuel

Municipal Gas Authority

Municipal Utility District

\begin{tabular}{|c|c|c|c|c|}
\hline $\begin{array}{c}1994 \\
\text { Jan.-Mar. }\end{array}$ & $\begin{array}{c}1994 \\
\text { Apr.-Jun. }\end{array}$ & $\begin{array}{c}1994 \\
\text { Jul.-Sep. }\end{array}$ & $\begin{array}{c}1994 \\
\text { Oct.-Dec. }\end{array}$ & $\begin{array}{c}1995 \\
\text { Jan.-Mar }\end{array}$ \\
\hline 0 & 161 & 502 & 2281 & 5224 \\
\hline 8 & 0 & 0 & 0 & 0 \\
\hline 0 & 0 & 15 & 18 & 17 \\
\hline 0 & 8 & 0 & 0 & 0 \\
\hline 0 & 0 & 267 & 0 & 0 \\
\hline 147 & 131 & 159 & 117 & 269 \\
\hline 84 & 60 & 29 & 55 & 50 \\
\hline 2 & 2 & 2 & 2 & 2 \\
\hline 0 & 0 & 25 & 0 & 0 \\
\hline 78 & 1109 & 1296 & 1368 & 973 \\
\hline 731 & 296 & 0 & 0 & 1532 \\
\hline 24 & 13 & 18 & 16 & 6 \\
\hline 0 & 0 & 0 & 0 & 57 \\
\hline 0 & 0 & 0 & 0 & 3 \\
\hline 88 & 88 & 88 & 34 & 61 \\
\hline 2 & 2 & 2 & 2 & 2 \\
\hline 0 & 0 & 54 & 0 & 67 \\
\hline 5 & 5 & 6 & 6 & 5 \\
\hline 0 & 0 & 0 & 1 & 0 \\
\hline 3 & 4 & 4 & 4 & 3 \\
\hline 8 & 0 & 0 & 301 & 317 \\
\hline 0 & 0 & 41 & 21 & 6 \\
\hline 542 & 0 & 203 & 253 & 189 \\
\hline 308 & 102 & 0 & 15 & 68 \\
\hline 1007 & 502 & 722 & 467 & 459 \\
\hline 6607 & 96 & 1143 & 2531 & 7782 \\
\hline 0 & 0 & 634 & 0 & 74 \\
\hline 409 & 633 & 949 & 2190 & 1407 \\
\hline 886 & 90 & 106 & 384 & 0 \\
\hline 533 & 0 & 0 & 770 & 506 \\
\hline 448 & 0 & 0 & 0 & 0 \\
\hline 0 & 0 & 0 & 30 & 0 \\
\hline 0 & 74 & 0 & 499 & 1119 \\
\hline 197 & 0 & 0 & 1 & 733 \\
\hline 0 & 147 & 563 & 522 & 544 \\
\hline 651 & 266 & 0 & 0 & 0 \\
\hline 978 & 890 & 358 & 409 & 565 \\
\hline 0 & 0 & 0 & 152 & 701 \\
\hline 3198 & 6174 & 2419 & 3357 & 4630 \\
\hline 0 & 381 & 0 & 0 & 11 \\
\hline 6307 & 0 & 311 & 0 & 0 \\
\hline 1217 & 670 & 753 & 1194 & 1597 \\
\hline 11 & 0 & 0 & 0 & 0 \\
\hline 0 & 0 & 33 & 35 & 25 \\
\hline 0 & 0 & 39 & 0 & 0 \\
\hline 385 & 0 & 0 & 0 & 0 \\
\hline 0 & 0 & 0 & 0 & 604 \\
\hline 0 & 59 & 0 & 0 & 0 \\
\hline 5 & 0 & 0 & 0 & 0 \\
\hline 0 & 0 & 0 & 281 & 469 \\
\hline
\end{tabular}


PURCHASERS OF SHORT-TERM GAS Estimated Volumes Imported (MMCF)

Murphy Gas Gathering

Murphy Plywood

N.Y. State Electric \& Gas

National Fuel Gas Distribution

National Fuel Resources

National Gas \& Electric

National Gas Resources

National Gas Services

National Steel Corporation

Natural Gas Clearinghouse

Natural Gas Pipeline

Natural Gas Resources

Neste Oy

Neste Trading USA Inc.

Nevada Power

New England Electric

New England Power Company

New Jersey Natural Gas

New York Power Authority

Niagara Mohawk

Nipsco Energy Trading Corporation

NorAm Energy Services

Norcen Energy Resources

Norfolk Paper Company

Norstar Energy

North American Chemical Company

North American Resources Company

North Atlantic Utilities

North Canadian Marketing Corp.

North Coast Energy, Inc.

North East Heat \& Light

Northeast Combustion Turbine

Northeast Heat \& Light

Northeast Utilities

Northem Illinois Gas

Northem Minnesota Utilities

Northem Natural Gas

Northem States Power (MN)

Northem States Power (WI)

Northem States Power Company

Northem States Power Gas Utility

Northem Utilities, Inc.

Northridge Gas Marketing Inc.

Northridge Petroleum Marketing U.S., Inc.

Northwest Alloys

Northwest Aluminum Company

Northwest Natural Gas Company

Northwest Pipeline

Northwestem Public Service Company

Norwood School Board
1994

Jan.-Mar

293

0

338

773

400

0

2465

0

1618

5702

110

181

1411

84

0

383

298

423

0

272

9

0

0

64

0

0

177

0

987

0

32

0

0

0

11121

1983

362

2154

318

669

16

127

0

0

256

100

1072

322

83

2
1994

Apr.-Jun.

296

0

458

0

85

0

2939

0

1668

5185

0

0

479

291

0

0

0

560

0

21

10

0

0

65

0

4

854

0

654

0

0

0

0

$$
0
$$

7659

395

0

2158

277

359

0

59

0

0

216

82

1319

0

85

2
1994
Jul.-Sep.

1994
Oct.-Dec

1995

Jan-Mar.

302
18

688

0

261

0

3136

0
1586

1586

12267

0

653

299

0

0

0

53

0

353

0

0

85

2

65

0

0

492

108

1546

0

0

18

0

795

9195

1465

113

2291

429

0

0

0

0

74

250

88

1010

215

84

2
0
110

0

703

0
2819

197

1736

6116

0

0

870

33

5

533

1266

0

376

205

0

74

0

65

0

0

409

0

1039

153

0

0

0

900

2663

1964

28

960

393

387

0

58

0

36

86

81

2440

30

29

1

$$
\begin{array}{r}
0 \\
0 \\
0 \\
337 \\
0 \\
155 \\
3975 \\
0 \\
2189 \\
7014 \\
0 \\
0 \\
813 \\
0 \\
0 \\
347 \\
1498 \\
0 \\
10 \\
0 \\
0 \\
425 \\
1 \\
61 \\
33 \\
0 \\
343 \\
0
\end{array}
$$


Norwood-Norfolk School

Novagas Clearinghouse

Nurserymen's Exchange

O \& R Energy

O \& R Marketing

OCM Boces

OLS Energy-Berkeley

Occidental

Ocean State Power

Ocean State Power II

Ogdensboro

Ogdensburg School District

Otympic Energy, Inc.

Olympic Fuels, Inc.

Omaha Utilities District

Orange \& Rockland Marketing

Orange \& Rockland Utilities

Ore-lda

Oroweat

Oryx Gas Marketing Company

PACCO

PG\&E Electric Supply Business Unit

PG\&E Gas Supply Business Unit

PSNC Production

Pabco Roofing

Pacific Gas \& Electric (UEG)

Pacific Gas \& Electric Company

Pacific Gas \& Energy

Pacific Gas Transmission

Pacific Gas Transmission (Linepack)

Pacific Northern Oil

Paladin

Pan-Alberta Gas (U.S.) Inc.

Pancanadian Petroleum Ltd.

Panhandle Trading Company

Paragon Gas Marketing

Pasco Cogen. Ltd.

Pawtucket Power Associates

Penn. Energy Marketing

Peoples Gas \& Light

Peoples Natural Gas Company

Pepperell Power Assoc. - Hydra Co.

Pepperell Power Associates, L.P.

Perry Gas Company

Petro Canada Hydrocarbons

Petro Source Gas Ventures

Phibro Energy USA Inc.

Phibro Oil \& Gas Inc.

Philadelphia Electric Company

Philadelphia Gas Works

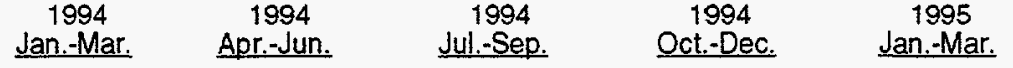

2
0

56

224

381

53496

29

6367

9801

0

2

0

641

0

0

265

0

0

54

0

0
1611

465

0

0

1360

5

9

1764

596

50
198

2

154

0

0

0

0

0

911

0

0

0

2268

32

15

140

0$$
0
$$$$
19
$$$$
74
$$$$
0
$$$$
0
$$$$
0
$$$$
0
$$$$
0
$$$$
0
$$$$
0
$$$$
44
$$$$
0
$$$$
92
$$$$
0
$$$$
423
$$$$
87
$$$$
0
$$$$
0
$$$$
289
$$$$
473
$$$$
52063
$$$$
0
$$$$
2888
$$$$
15412
$$$$
140
$$$$
1
$$$$
0
$$$$
0
$$$$
0
$$$$
0
$$$$
1154
$$$$
1513
$$$$
0
$$$$
0
$$$$
0
$$$$
0
$$$$
0
$$$$
1237
$$$$
0
$$$$
\begin{array}{r}
0 \\
53
\end{array}
$$$$
1350
$$$$
392
$$$$
970
$$$$
228
$$$$
0
$$$$
0
$$

50665 
PURCHASERS OF SHORT-TERM GAS Estimated Volumes Imported (MMCF)

Phoenix Chemical

Pictsweet

Piedmont Natural Gas Company

Plum Creek Manufacturing

Poco Petroleum

Poppy Foods

Port of Portland

Portland General Electric Company

Potlatch Corporation

Potsdam Central School

Potsdam College

Potsdam Hardwoods

Potsdam Laundry

Potters Industries

Power City Partners, L.P

Prairieland Energy Marketing

Premier Edible Oils

Progas

Public Senvice Electric \& Gas

Public Service of Colorado

Public Service of North Carolina

Puget Sound Power \& Light

Questar Pipeline Company

Rainier Brewery

Reatta

Redwood Resources Inc

Reed College

Renaissance Energy (U.S.) Inc.

Reynolds Metal Corporation

Rhone-Poulenc

Rocky Mountain Natural Gas

Rogers Foods

Rome Family YMCA

Roseburg Lumber

Ross Simmons Hardwood

Royale Natural Gas

SCANA

SEMCO Energy Services

SL Knit

SUN

SUNY - N.Y

Sacremento Municipal

Salmon Resources

Salwasser

San Diego Gas \& Electric Company

Santanna Natural Gas

Seattle Steam

Selkirk Cogeneration Partners

Shell Oil Company

Sierra Pacific Industries

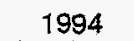

Jan.-Mar.

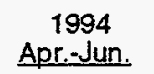

1994
Jull.-Sep.

1994
Oct.-Dec.

1995
Jan.-Mar.

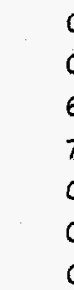

353

0

0

10

0

0

0

3131

331

6

46

3

2

52

0

0

53

0

213

0

281

2

1230

0

0

624

0

0

6

374

17

0

11

10

0

0

0

0

0

0

72

0

2696

0

742

0

273

23

335

0
787

289

6

47

4

2

62

0

0

48

0

324

30

754

0

0

0

0

336

0

0

0

$\theta$

0

142

0

15

0

0

0

0

0

0

65

6

2745

0

1406

0

245

0

551

\section{0}

29

0

9

459

8

9

1533

344

6

49

4

2

68

0

0

47

0

68

0

766

323

0

0

0

703

3

0

72

106

0

166

0

48

0

23

0

0

0

0

0

0

3604

14

2921
96

40

166

16

0

10

38

4214

436

6

48

1

1

64

25

14

48

310

550

0

220

44

0

34

371

540

20

0

166

145

0

129

0

14

0

163

24

0

0

34

606

0

2843

36

1082

34

162

308

570

92

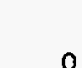

43

806

39

54

1

51

2836

473

5

47

0

62

44

3

0

1023

92

0

0

0

0

47

0
297

19

10348

109

190

14

1

0

40

29

40 
Sierra Pacific Power Company

Simpson Paper

Simpson Tacoma Kraft Company

Sioux Pointe Inc.

Smurfit Newsprint Corporation

Southeastem Michigan Gas Company

Southem Califomia Edison Company

Southem Califomia Gas Company

Southem Connecticut Gas Company

Southwest Gas Corporation

St. Joseph's Hospital

St. Lawrence University

St. Regis Nursing Home

Stella Cheese Company

Stokely USA

Stone Container Corporation

Sun Chemical

Sun Refining

Sunpacific Energy Management

Sunrise Energy

Sunrise Marketing

Talisman Energy

Tarrant Resources

Teco Gas Marketing

Teledyne

Tenaska Gas Company

Tenaska Marketing Ventures

Tenaska Washington Partners

Tenneco Gas Marketing

Tennessee Gas Pipeline Company

Terra Industries

Terra International

TexPar Energy, Inc.

Texaco Gas Marketing Inc.

Texaco Natural Gas

Texas Ohio, West Inc.

Texas-Ohio Gas, Inc.

The Montana Power Company

Torch Gas L.C.

Tosco Energy

Tosco Northwest

Tosco Refining Company

Transco Energy Marketing Company

Transok

Tristar Gas Marketing

Trunkline Gas Company

Twister Transmission Company

U.S. Energy

U.S. Gas Transmission

U.S. Gas Transportation Inc.

\begin{tabular}{|c|c|c|c|c|}
\hline $\begin{array}{c}1994 \\
\text { Jan.-Mar. }\end{array}$ & $\begin{array}{c}1994 \\
\text { Apr-Jun. }\end{array}$ & $\begin{array}{c}1994 \\
\text { Jul.-Sep. }\end{array}$ & $\begin{array}{c}1994 \\
\text { Oct.-Dec. }\end{array}$ & $\begin{array}{c}1995 \\
\text { Jan.-Mar. }\end{array}$ \\
\hline 3462 & 3284 & 4773 & 3818 & 4372 \\
\hline 0 & 0 & 0 & 366 & 451 \\
\hline 0 & 0 & 0 & 261 & 439 \\
\hline 1516 & 834 & 0 & 293 & 0 \\
\hline 0 & 0 & 0 & 6 & 132 \\
\hline 36 & 0 & 0 & 0 & 0 \\
\hline 4802 & 5590 & 5573 & 4321 & 0 \\
\hline 44 & 49 & 0 & 310 & 51 \\
\hline 11 & 0 & 0 & 0 & 4 \\
\hline 813 & 546 & 18 & 747 & 446 \\
\hline 11 & 0 & 0 & 0 & 0 \\
\hline 32 & 32 & 36 & 33 & 169 \\
\hline 1 & 1 & 1 & 2 & 1 \\
\hline 17 & 18 & 18 & 18 & 17 \\
\hline 0 & 0 & 59 & 46 & 7 \\
\hline 34 & 184 & 228 & 347 & 346 \\
\hline 0 & 39 & 33 & 0 & 0 \\
\hline 0 & 0 & 0 & 0 & 86 \\
\hline 747 & 324 & 171 & 129 & 0 \\
\hline 2490 & 4284 & 4047 & 1385 & 1222 \\
\hline 2 & 0 & 0 & 0 & 0 \\
\hline 0 & 0 & 5 & 0 & 0 \\
\hline 2 & 0 & 0 & 0 & 0 \\
\hline 0 & 0 & 143 & 658 & 1028 \\
\hline 77 & 63 & 62 & 62 & 28 \\
\hline 158 & 714 & 80 & 100 & 102 \\
\hline 5844 & 4938 & 6070 & 6444 & 6024 \\
\hline 0 & 208 & 283 & 95 & 0 \\
\hline 195 & 1123 & 620 & 871 & 723 \\
\hline 0 & 0 & 1183 & 0 & 583 \\
\hline 1957 & 59 & 0 & 0 & 0 \\
\hline 0 & 1767 & 1521 & 1133 & 0 \\
\hline 0 & 783 & 30 & 0 & 0 \\
\hline 323 & 702 & 49 & 37 & 0 \\
\hline 0 & 146 & 0 & 0 & 0 \\
\hline 441 & 199 & 0 & 0 & 83 \\
\hline 0 & 113 & 737 & 606 & 404 \\
\hline 309 & 58 & 0 & 634 & 698 \\
\hline 25 & 0 & 0 & 0 & 0 \\
\hline 139 & 0 & 29 & 0 & 62 \\
\hline 0 & 0 & 29 & 153 & 105 \\
\hline 5 & 244 & 0 & 0 & 0 \\
\hline 0 & 0 & 340 & 1007 & 1595 \\
\hline 0 & 0 & 0 & 81 & 0 \\
\hline 1032 & 684 & 646 & 153 & 173 \\
\hline 6 & 1955 & 0 & 0 & 0 \\
\hline 783 & 0 & 0 & 0 & 0 \\
\hline 0 & 0 & 0 & 0 & 973 \\
\hline 0 & 0 & 14 & 0 & 0 \\
\hline 57 & 364 & 232 & 672 & 722 \\
\hline
\end{tabular}


Page - 68

PURCHASERS OF SHORT-TERM GAS

Estimated Volumes Imported (MMCF)

1994

Jan-Mar.

1994

Apr.-Jun.

1994

1994

Oct.-Dec

1995

Jan.-Mar.

UGI Utilities

US Energy

Unicorp Energy Inc.

Unicorp United

Unifield Natural Gas Group Inc.

Union Gas Limited

Union Oil

Union Pacific Fuels

Union Pacific Resources

United City

United Cogen

United Helpers Managernent Company

Universal Frozen Foods

Universal Resources

Unocal

Utah Gas Services

Utilicorp United

Valero Industrial Gas

Vanalco

Various Midwest Customers

Various NY State Markets

Various New England Markets

Various Northeastem U.S. Customers

Various PA State Markets

Vastar Gas

Vermont Gas Systems, Inc.

Viking Gas Transmission

Village of Potsdam Housing Auth.

Virginia Power

Vlasic Foods Inc.

Volunteer Energy Corporation

WP Natural Gas

Wasatch Oil \& Gas

Wascana Energy Marketing

Washington Beef

Washington Energy Gas Marketing Company

2231

Western Gas Marketing Inc.

Western Gas Resources

Western Gas Services

Western Sugar

Westvaco

Weyerhauser

Wickford Energy Marketing Inc.

Willamette

Williston Basin Interstate Pipeline
0

0

0

107

1490

3

36

227

0

0

4

186

192

0

0

0

0

0

170

237

0

718

155

296

0

3

0

0

0

182

0

10

0

46

5382

1385

1083

370

1310

2666

\section{-Sep.}

1475

0
1641

0

0

0

5

119

1079

0

0

2469

0

0

0

223

0

0

0

0

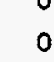

0
4

4
6

0

0

131

0

0

1902

5369

1273

269

389

2414

155

1131

0
0

0

0

1136

5

11

0
15

303

0

729

0

1481

321

0

280

0

0

1744

1

1038

0

238

72

3

78

1243

0

0

1310

88

288

4

4
290

0

87

0

0

35

0

4

0

29

0

149

59

0

0

2724

7566

1744

0

159

3511

283

171

150

0

323

1133

0

255

80

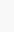


PURCHASERS OF SHORT-TERM GAS

Estimated Volumes Imported (MMCF)

Windward Energy \& Marketing Inc.

Wisconsin Gas

Wisconsin Power \& Light

Wisconsin Public Service Corp.

Yankee Gas Services

Zacky Farms

Totals
1994

Jan.-Mar.

2

11392

750

718

84

0

$\underline{309196}$

\author{
1994 \\ Apr.-Jun.
}

3

175

551

255

0

0

$\underline{268915}$
1994

Jul.-Sep.

1158

487

509

0

0

21

$\underline{305473}$
1994

Oct.-Dec.

8

433

1136

0

0

34

$\underline{325336}$
1995 Jan.-Mar.

2
78
090
16
12
34

383193 
SHORT-TERM SALES

YEAR: 1995 Quarter: One

Estimated Volumes (MMCF) \& Prices ( $/$ MMBTU)
Importer

Seller

Purchaser / End User

AIG TRADING CORPORATION AIG Trading Corporation

MichCon Storage

AIG TRADING CORPORATION AIG Trading Corporation

MichCon Storage

AIG TRADING CORPORATION AIG Trading Corporation

MichCon Storage

ALTRESCO PITTSFIELD, L.P.

New England Power

Altresco Pittsfield, L.P.

ALTRESCO PITTSFIELD, L.P.

Northridge Gas Marketing

Altresco Pittsfield, L.P.

AMERADA HESS COFIPORATION Amerada Hess Canada

AIG Trading Corporation

AMERADA HESS COFPORATION Amerada Hess Canada

Amerada Hess

Port of Morgan, MT

$51 \quad 1.51$

- N/A

o N/A

$51 \quad 1.51$

AMERADA HESS CORPORATION Amerada Hess Canada

Aquila Energy Marketing

AMERADA HESS CORPORATION Amerada Hess Canada CNG Gas Services

Port of Morgan, MT

- N/A

- N/A

$39 \quad 1.24$

$39 \quad 1.24$

AMERADA HESS CORPORATION Amerada Hess Canada

Eastex Energy

Port of Morgan, MT

o N/A

- N/A

$50 \quad 1.24$

$50 \quad 1.24$

AMERADA HESS CORPORATION Amerada Hess Canada Enron Gas Marketing
Port of Morgan, MT
$100 \quad 1.51$

- N/A

$52 \quad 1.24$

$152 \quad 1.42$ 
Importer

Purchaser/End User

AMERADA HESS CORPORATION

Amerada Hess Canada

Natural Gas Clearinghouse

AMERADA HESS CORPORATION

Amerada Hess Canada

NorAm Energy Services

AMERADA HESS CORPORATION Amerada Hess Canada

Teco Gas Marketing

Port of Morgan, MT

$0 \quad N / A$

$40 \quad 1.26$

- N/A

$40 \quad 1.26$

AMERADA HESS CORPORATION

Amerada Hess Canada

Tenaska Marketing Ventures

Port of Morgan, MT

$0 \quad N / A$

$95 \quad 1.26$

$2 \quad 1.24$

$97 \quad 1.26$

AMERADA HESS CORPORATION

Amerada Hess Canada

Westcoast Gas Services (U.S.A.) Inc

Port of Morgan, MT

$48 \quad 1.51$

$0 \quad$ N/A

- N/A

48

1.51

AMERADA HESS CORPORATION

Amerada Hess Canada

Western Gas Marketing Inc.

Port of Morgan, MT

$34 \quad 1.51$

$0 \quad$ N/A

$51 \quad 1.24$

$85 \quad 1.35$

AMOCO CANADA MARKETING CORPORATION

Amoco Canada Marketing Corporation

Amoco Trading

Eastport, Idaho

1158

1.15

952

0.74

$819 \quad 0.87$

2929

0.94

AMOCO CANADA MARKETING CORPORATION

Amoco Canada Marketing Corporation

Associated Gas Services, Inc.

Eastport, Idaho

AMOCO CANADA MARKETING CORPORATION

Amoco Canada Marketing Corporation

Enron Gas Marketing

Eastport, Idaho

AMOCO CANADA MARKETING CORPORATION

Amoco Canada Marketing Corporation

IGI Resources

Eastport, Idaho

19

0.81

$20 \quad 0.87$

403

0.91

AMOCO CANADA MARKETING CORPORATION

Amoco Canada Marketing Conporation

Pacific Gas \& Electric Company

Eastport, Idaho
724

0.85

$819 \quad 0.87$

2038

0.90 
SHORT-TERM SALES

YEAR: 1995 Quarter: One

Estimated Volumes (MMCF) \& Prices ( $\$$ / MMBTU)
Importer

Seller

Purchaser / End User
Point

of

Entry

AMOCO CANADA MAFIKETING CORPORATION

Amoco Canada Marketing Corporation

Tristar Gas Marketing

Eastport, Idaho

95

0.73

January

Vol. Price

February
vol.

Vol. Price

March

Vol. Price

Quarterty

Total

Vol. ${ }^{\text {Avg. }}$ Price

AMOCO CANADA MARKETING CORPORATION

Amoco Canada Marketing Corporation

U.S. Gas Transportation Inc.

Eastport, Idaho

13

0.69

$4 \quad 0.67$

- N/A

17

0.69

AMOCO CANADA MARKETING CORPORATION

Amoco Canada Marketing Corporation

Westcoast Gas Services (U.S.A.) Inc

Eastport, Idaho

182

1.03

69

0.75

- N/A

251

0.95

AMOCO ENERGY TRADING CORPORATION

Amoco Energy Trading Corporation

Amoco Trading

Eastport, Idaho

$181 \quad 1.57$

- N/A

310

1.15

491

1.30

AMOCO ENERGY TRADING CORPORATION

Amoco Energy Trading Corporation

Amoco Trading

Port of Morgan, MT

O N/A

$0 \quad N / A$

10

1.16

10

1.16

AMOCO ENERGY TRADING CORPORATION

Amoco Energy Trading Corporation

Amoco Trading

Waddington, NY

O N/A

61

2.23

94

1.91

154

2.04

AMOCO ENERGY TRADING CORPORATION

PEMEX

Amoco Trading

Hidalgo, Texas

$0 \quad N / A$

o N/A

150

1.48

150

1.48

ANR PIPELINE COMPANY

Western Gas Marketing Limited

ANR Pipeline Company

Noyes, Minnesota

59

3.18

$541 \quad 2.90$

599

2.82

1740

2.97

ARCO PRODUCTS COMPANY

AGS Gas Services

Arco Products Comparyy

Sumas, Washington

$10 \quad 5.47$

$24 \quad 2.38$

O N/A

34

3.26

ARCO PRODUCTS COMPANY

CanWest Marketing

Arco Products Company

Sumas, Washington

$256 \quad 1.71$

256

1.29

178

1.14

689

1.41

ARCO PRODUCTS COMPANY

Mobil Oil Canada Ltd.

Arco Products Company

Sumas, Washington

o N/A

o N/A

14

1.00
$14 \quad 1.00$ 


\section{SHORT-TERM SALES \\ YEAR: 1995 Quarter: One \\ Estimated Volumes (MMCF) \& Prices (\$ / MMBTU)}

Importer

Seller

Purchaser / End User

ARCO PRODUCTS COMPANY

Shell Canada Limited

Arco Products Company

ARCO PRODUCTS COMPANY

Westcoast Gas Services inc.

Arco Products Company

ASSOCIATED GAS SERVICES, INC. Associated Gas Services, Inc.

Anodizing

Sumas, Washington

Sumas, Washington

ASSOCIATED GAS SERVICES, INC

Associated Gas Services, Inc.

Aquila Energy Marketing

ASSOCIATED GAS SERVICES, INC

Associated Gas Services, Inc.

Associated Gas Services, Inc.

Eastport, Idaho

ASSOCIATED GAS SERVICES, INC Associated Gas Services, Inc.

Atochem

Sumas, Washington

$0 \quad$ N/A

$27 \quad 1.03$

$4 \quad 1.00$

$31 \quad 1.03$

ASSOCIATED GAS SERVICES, INC. Associated Gas Services, Inc.

Baker Commodities

Sumas, Washington

Sumas, Washington

$17 \quad 1.40$

14. $\quad 1.03$

$15 \quad 1.00$

$46 \quad 1.16$

ASSOCIATED GAS SERVICES, INC. Associated Gas Services, Inc.

Cabot Oil

Sumas, Washington

- N/A

$0 \quad$ N/A

$8 \quad 1.00$

$8 \quad 1.00$

ASSOCIATED GAS SERVICES, INC. Associated Gas Services, Inc.

Cascade Natural Gas

Sumas, Washington

- N/A

140

1.03

$0 \quad$ N/A

$140 \quad 1.03$

ASSOCIATED GAS SERVICES, INC. Associated Gas Services, Inc.

Cascade Steel
Sumas, Washington
$13 \quad 1.40$

1.03

1.00

$81 \quad 1.08$ 


\section{SHORT-TERM SALES \\ YEAR: 1995 Quarter: One \\ Estimated Volumes (MMCF) \& Prices (\$ / MMBTU)}

Importer

Seller

Purchaser / End User

\section{Point \\ of}

Entry

Sumas, Washington

Sumas, Washington

Associated Gas Services, Inc.

Continental Baking

ASSOCIATED GAS SERVICES, INC. Associated Gas Services, Inc.

Darigold Inc.

ASSOCIATED GAS SERVICES, INC

Associated Gas Services, Inc.

Domtar Gypsum

Sumas, Washington

ASSOCIATED GAS SERVICES, INC.

Associated Gas Services, Inc.

Evanite Fiber

Sumas, Washington

ASSOCIATED GAS SERVICES, INC.

Associated Gas Services, Inc.

Flavorland Foods

Sumas, Washington

ASSOCIATED GAS SERVICES, INC. Associated Gas Services, Inc.

Fujitsu

Sumas, Washington

ASSOCIATED GAS SERVICES, INC.

Associated Gas Services, Inc.

General Chemical

Sumas, Washington

ASSOCIATED GAS SERVICES, INC.

Associated Gas Services, Inc.

Georgia Pacific Compration

ASSOCIATED GAS SERVICES, INC.

Associated Gas Services, Inc.

Hytek Finishes

Sumas, Washington

$6 \quad 1.40$

$4 \quad 1.03$

$5 \quad 1.00$

16

1.16

ASSOCIATED GAS SERVICES, INC. Associated Gas Services, Inc.

IGI Resources

January

February

Vol. Price

March

Vol. Price

Quarterly

Total

Vol. Price

$27 \quad 1.40$

$22 \quad 1.03$

$23 \quad 1.00$

72

1.16

$110 \quad 1.40$

$104 \quad 1.03$

$114 \quad 1.00$

328

1.14

$9 \quad 1.40$

$67 \quad 1.03$

85

161

1.04

$9 \quad 1.40$

$10 \quad 1.03$

$15 \quad 1.00$

34

1.11

$3 \quad 1.40$

$2 \quad 1.03$

$0 \quad$ N/A

$5 \quad 1.22$

Sumas, Washington

$37 \quad 2.80$

$107 \quad 2.06$

$20 \quad 2.00$

164

2.22

Sumas, Washington
$36 \quad 1.40$

$448 \quad 1.03$

1.00

1028

1.03 
SHORT-TERM SALES

YEAR: 1995 Quarter: One

Estimated Volumes (MMCF) \& Prices (\$ / MMBTU)
Importer

Seller

Purchaser/End User

ASSOCIATED GAS SERVICES, INC.

Associated Gas Services, Inc.

Inland Pacific

Sumas, Washington

Sumas, Washington

Associated Gas Services, Inc.

James River Corporation

ASSOCIATED GAS SERVICES, INC.

Associated Gas Services, Inc.

Kalama Chemical

Sumas, Washington

ASSOCIATED GAS SERVICES, INC

Associated Gas Services, Inc.

McCall Oil

Sumas, Washington

ASSOCIATED GAS SERVICES, INC.

Associated Gas Services, Inc.

National Gas Resources

Sumas, Washington

$0 \quad$ N/A

$0 \quad N / A$

$93 \quad 1.00$

$93 \quad 1.00$

ASSOCIATED GAS SERVICES, INC. Associated Gas Services, Inc.

Natural Gas Clearinghouse

Sumas, Washington

Sumas, Washington

ASSOCIATED GAS SERVICES, INC.

Associated Gas Services, Inc.

Northwest Aluminum Company

ASSOCIATED GAS SERVICES, INC.

Associated Gas Services, Inc.

Northwest Natural Gas Company

Sumas, Washington

- N/A

$66 \quad 1.03$

- N/A

66

1.03

ASSOCIATED GAS SERVICES, INC. Associated Gas Services, Inc.

Occidental

Sumas, Washington

0 N/A

11.03

29

1.00

$30 \quad 1.00$

ASSOCIATED GAS SERVICES, INC. Associated Gas Services, Inc.

Pabco Roofing

Sumas, Washington

$3 \quad 1.40$

$4 \quad 1.03$

$5 \quad 1.00$

12

1.12

ASSOCIATED GAS SERVICES, INC. Associated Gas Services, Inc.

Petro Canada Hydrocarbons

Sumas, Washington
$465 \quad 1.40$

$0 \quad$ N/A

O N/A

465

1.40 


\section{SHORT-TERM SALES \\ YEAR: 1995 Quarter: One \\ Estimated Volumes (MMCF) \& Prices (\$ / MMBTU)}

Importer

Seller

Point

of

Purchaser / End User

Entry

January
Vol. Price

February
Vol. Price

March

Vol. Price

Quarterty
Total

Avg.

ASSOCIATED GAS SERVICES, INC.

Associated Gas Services, inc.

Pictsweet

Sumas, Washington

$16 \quad 1.40$

$12 \quad 1.03$

$14 \quad 1.00$

43

1.16

ASSOCIATED GAS SERVICES, INC.

Associated Gas Services, Inc.

Port of Portland

Sumas, Washington

$21 \quad 1.40$

$15 \quad 1.03$

$15 \quad 1.00$

51

1.18

ASSOCIATED GAS SERVICES, INC.

Associated Gas Services, Inc.

Rainier Brewery

Sumas, Washington

$17 \quad 1.40$

$12 \quad 1.03$

$17 \quad 1.00$

$47 \quad 1.16$

ASSOCIATED GAS SERVICES, INC.

Associated Gas Services, Inc.

Reed College

Sumas, Washington

$8 \quad 1.40$

$6 \quad 1.03$

$6 \quad 1.00$

19

1.17

ASSOCIATED GAS SERVICES, INC.

Associated Gas Services, Inc.

Reynolds Metal Corporation

Sumas, Washington

- N/A

$46 \quad 1.03$

$64 \quad 1.00$

109

1.01

ASSOCIATED GAS SERVICES, INC.

Associated Gas Services, Inc.

Rocky Mountain Natural Gas

Sumas, Washington

- N/A

$14 \quad 1.03$

O N/A

14

1.03

ASSOCIATED GAS SERVICES, INC. Associated Gas Services, Inc.

Ross Simmons Hardwood

Sumas, Washington

o N/A

$14 \quad 1.03$

$14 \quad 1.00$

29

1.01

ASSOCIATED GAS SERVICES, INC.

Associated Gas Services, Inc.

Seattle Steam

Sumas, Washington

$41 \quad 1.40$

62. 1.03

$15 \quad 1.00$

117

1.15

ASSOCIATED GAS SERVICES, INC.

Associated Gas Services, Inc.

Sierra Pacific Power Company

Sumas, Washington

$0 \quad$ N/A

$19 \quad 1.03$

$17 \quad 1.00$

35

1.02

ASSOCIATED GAS SERVICES, INC.

Associated Gas Services, inc.

Smurfit Newsprint Corporation

Sumas, Washington

$6 \quad 1.40$

$5 \quad 1.03$

$121 \quad 1.00$

132

1.02

ASSOCIATED GAS SERVICES, INC. Associated Gas Services, Inc.

Stokely USA

Sumas, Washington

$4 \quad 1.40$

$3 \quad 1.03$

$0 \quad 1.00$

7 
SHORT-TERM SALES

YEAR: 1995 Quarter: One

Estimated Volumes (MMCF) \& Prices (\$ / MMBTU)
Importer

Seller

Purchaser / End User

ASSOCIATED GAS SERVICES, INC. Associated Gas Services, Inc.

Teledyne

ASSOCIATED GAS SERVICES, INC. Associated Gas Services, Inc.

Tristar Gas Marketing

Sumas, Washington

Detroit, Michigan

Sumas, Washington

Sumas, Washington

Associated Gas Services, Inc.

Washington Beef

ASSOCIATED GAS SERVICES, INC.

Associated Gas Services, Inc.

Washington Natural Gas Company

ASSOCIATED GAS SERVICES, INC.

Associated Gas Services, Inc.

Washington Water Power Company

ASSOCIATED GAS SERVICES, INC.

Associated Gas Services, Inc.

Weyerhauser

Sumas, Washington

12

2.80

112

3.09

$114 \quad 3.00$

239

3.03

BAY STATE GAS COMPANY

Renaissance Energy Ltd.

Bay State Gas Company

BC GAS UTILITY LTD.

B.C. Gas Inc.

Universal Resources

Sumas, Washington

$0 \quad N / A$

76

0.91

$0 \quad N / A$

$\begin{array}{ll}76 & 0.91\end{array}$

BROAD STREET OIL \& GAS COMPANY

Pan-Alberta Gas Ltd.

Broad Street Oil \& Gas
Eastport, Idaho

$\begin{array}{llll}113 & 1.08 & 102 & 0.85\end{array}$

O N/A

214

0.97 
SHORT-TERM SALES

YEAR: 1995 Quarter: One

Estimated Volumes (MMCF) \& Prices (\$ / MMBTU)

Importer

Seller

Purchaser/End User
Point

Entry
January
Vol. Price

February

Vol. Price
Quarterly

Total
Avg.

Vol. Price

CABOT OIL \& GAS TRADING CORPORATION

Canadian Natural Resources Limited

Boeing Company

Sumas, Washington

- N/A

$138 \quad 1.64$

$149 \quad 1.64$

287

1.64

CABOT OIL \& GAS TRADING CORPORATION

Ocelot Energy, Inc.

Boeing Company

Sumas, Washington

$154 \quad 1.64$

$0 \quad$ N/A

- N/A

154

1.64

CANADA IMPERIAL OIL LIMITED

Imperial Oil Resources

Canada Imperial Oil

Eastport, Idaho

$203 \quad 1.69$

$141 \quad 1.38$

o N/A

$344 \quad 1.56$

CANADIANOXY MARKETING INC.

Canadian Occidental Petroleum Ltd.

Canadian Oxy Marketing inc.

Noyes, Minnesota

- N/A

206

1.10

$0 \quad$ N/A

206

1.10

CANSTATES GAS MARKETING (U.S.) Ltd.

CanStates Gas Marketing

Cook Inlet

Eastport, Idaho

$44 \quad 1.05$

O N/A

O N/A

$44 \quad 1.05$

CANSTATES GAS MARKETING (U.S.) Ltd.

CanStates Gas Marketing

Equitable Resources

Eastport, Idaho

111

1.15

O N/A

o N/A

111

1.15

CANSTATES GAS MARKETING (U.S.) Ltd.

CanStates Gas Marketing

Home Oil

Eastport, Idaho

$\begin{array}{llll}0 & \text { N/A } & 404 & 1.01\end{array}$

$452 \quad 1.00$

850

1.00

CANSTATES GAS MARKETING (U.S.) Ltd.

CanStates Gas Marketing

IGI Resources

Eastport, Idaho

67

1.12

$10 \quad 1.15$

- N/A

76

1.12

CANSTATES GAS MARKETING (U.S.) LId.

CanStates Gas Marketing

Pacific Gas \& Electric Company

Eastport, Idaho

21.08

128

1.15

$179 \quad 1.14$

309

1.14

CANSTATES GAS MARKETING (U.S.) Ltd.

CanStates Gas Marketing

Petro Source Gas Ventures

Eastport, Idaho

1301

1.71

809

1.01

89

3001

1.32

CANWEST GAS SUPPLYU.S.A., INC.

CanWest Gas Supply Inc.

American Hunter Energy

Eastport, Idaho

$91 \quad 0.89$

- N/A

91

0.89 
SHORT-TERM SALES

YEAR: 1995 Quarter: One

Estimated Volumes (MMCF) \& Prices ( $\$$ / MMBTU)
Importer

Seller

Purchaser / End User

CANWEST GAS SUPPLY U.S.A., INC. CanWest Gas Supply inc.

Cascade Natural Gas

CANWEST GAS SUPPLYU.S.A., INC CanWest Gas Supply Inc.

Chevron U.S.A. inc.

CANWEST GAS SUPPLY U.S.A., INC. CanWest Gas Supply Inc.

Coastal Gas Marketing Company

CANWEST GAS SUPPLYU.S.A., INC CanWest Gas Supply lnc

DAl Oildale

Eastport, Idaho

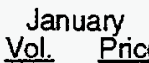

Eastport, Idaho

$119 \quad 1.06$

February
Vol. Price

Entry

Eastport, Idaho

Eastport, Idaho

$5 \quad 1.06$

$\begin{array}{ll}715 & 0.89\end{array}$

611

0.72

1331

0.81

CANWEST GAS SUPPLY U.S.A., INC. CanWest Gas Supply Inc.

Enron Gas Marketing

Eastport, Idaho

178

$0 \quad$ N/A

65

0.89

95

0.72

160

0.79

CANWEST GAS SUPPLY U.S.A., INC. CanWest Gas Supply Inc.

IGI Resources

Eastport, Idaho

110

1104

1.78

1088

1.44

3295

1.43

CANWEST GAS SUPPLY U.S.A., INC. CanWest Gas Supply lnc.

Interenergy Corporation

CANWEST GAS SUPPLY U.S.A., INC.

CanWest Gas Supply Inc.

James River Corporation

Eastport, Idaho

170

2.12

174

1.78

203

1.44

546

1.76

CANWEST GAS SUPPLY U.S.A., INC. CanWest Gas Supply Inc.

Kimball Energy Corporation

CANWEST GAS SUPPLY U.S.A., INC. CanWest Gas Supply Inc.

Koch Gas Services Company

Eastport, Idaho

$30 \quad 1.06$

$0 \quad N / A$

104

0.72

134

0.80

CANWEST GAS SUPPLY U.S.A., INC. CanWest Gas Supply inc.

Masonite

Eastport, Idaho
$3 \quad 1.06$

65

0.89

- N/A

67

0.90 
SHORT-TERM SALES

YEAR: 1995 Quarter: One

Estimated Volumes (MMCF) \& Prices (\$ / MMBTU)
Importer

Seller

Purchaser / End User

CANWEST GAS SUPPLY U.S.A., INC. CanWest Gas Supply Iric.

Mock Resources

CANWEST GAS SUPPLY U.S.A., INC.

CanWest Gas Supply iric.

Natural Gas Clearinghouse

CANWEST GAS SUPPLY U.S.A., INC.

CanWest Gas Supply Inic.

Northwest Natural Gas Company

Eastport, Idaho

134

January

of

Entry

Eastport, Idaho

Eastport, Idaho

Eastport, Idaho

CanWest Gas Supply Inc.

Northwest Pipeline

CANWEST GAS SUPPLY U.S.A., INC.

CanWest Gas Supply Inc.

Pacific Gas \& Electric (IJEG)

CANWEST GAS SUPPLY U.S.A., INC.

CanWest Gas Supply Inc.

Pacific Gas Transmission

CANWEST GAS SUPPLY U.S.A., INC.

CanWest Gas Supply Inc.

Portland General Electric Company

Eastport, Idaho

870

2.12

643

0.89

183

0.72

1696

1.50

CANWEST GAS SUPPLY U.S.A., INC. CanWest Gas Supply Inc.

Royale Natural Gas

Eastport, Idaho

- N/A

$40 \quad 0.89$

o N/A

40

0.89

CANWEST GAS SUPPLY U.S.A., INC. CanWest Gas Supply Inc.

San Diego Gas \& Electric Company

CANWEST GAS SUPPLY U.S.A., INC.

CanWest Gas Supply Inc.

Sierra Pacific Power Company

Eastport, Idaho

O N/A

$0 \quad$ N/A

218

0.72

218

0.72

CANWEST GAS SUPPLY U.S.A., INC.

CanWest Gas Supply Inc.

Simpson Paper
Eastport, ldaho
2.12

16

1.78

105

0.72

451

1.67 


\section{SHORT-TERM SALES \\ YEAR: 1995 Quarter: One \\ Estimated Volumes (MMCF) \& Prices (\$ / MMBTU)}

Importer

Seller

Purchaser / End User

CANWEST GAS SUPPLY U.S.A., INC.

CanWest Gas Supply Inc.

Simpson Tacoma Kraft Company

CANWEST GAS SUPPLY U.S.A., INC

CanWest Gas Supply Inc.

Southwest Gas Corporation

CANWEST GAS SUPPLY U.S.A., INC. CanWest Gas Supply Inc.

Teco Gas Marketing

CANWEST GAS SUPPLY U.S.A., INC. CanWest Gas Supply Inc.

Tenaska Gas Company

CANWEST GAS SUPPLY U.S.A., INC. CanWest Gas Supply inc.

Tosco Northwest

Eastport, Idaho

CANWEST GAS SUPPLY U.S.A., INC CanWest Gas Supply Inc.

Union Pacific Fuels

Eastport, Idaho

$47 \quad 1.06$

- N/A

$0 \quad N / A$

$47 \quad 1.06$

CANWEST GAS SUPPLY U.S.A., INC. CanWest Gas Supply inc.

United Cogen

Eastport, Idaho

$61 \quad 1.06$

$11 \quad 0.89$

$0 \quad$ N/A

72

1.04

CANWEST GAS SUPPLY U.S.A., INC CanWest Gas Supply inc.

Universal Resources

Eastport, Idaho

$297 \quad 1.06$

159

0.89

595

0.72

1051

0.84

CANWEST GAS SUPPLY U.S.A., INC.

CanWest Gas Supply Inc.

Washington Natural Gas Company

Eastport, Idaho

$1548 \quad 1.06$

1478

0.89

1650

1.44

4676

1.14

CANWEST GAS SUPPLY U.S.A., INC.

CanWest Gas Supply Inc.

Washington Water Power Company

Eastport, Idaho

- N/A

$0 \quad$ N/A

25

0.72

25

0.72

CANWEST GAS SUPPLY U.S.A., INC.

CanWest Gas Supply Inc.

Watson Cogeneration

Eastport, Idaho
$0 \quad$ N/A

$0 \quad N / A$

149

0.72

149

0.72 
SHORT-TERM SALES

YEAR: 1995 Quarter: One

Estimated Volumes (MMCF) \& Prices (\$ / MMBTU)

Importer

Seller

Purchaser / End User

CASCADE NATURAL GAS CORPORATION

Associated Gas Services, Inc.

Cascade Natural Gas

Sumas, Washington

Sumas, Washington

CASCADE NATURAL GIAS CORPORATION

CanWest Gas Supply Inc.

Cascade Natural Gas

Point

Entry

January
Vol. Price

February
Vol. Price

March
Vol. Price

$108 \quad 4.15$

$223 \quad 4.19$

$255 \quad 4.56$

586

4.34

CASCADE NATURAL GAS CORPORATION

Equitable

Cascade Natural Gas

Eastport, Idaho

O N/A

o N/A

$13 \quad 0.96$

13

0.96

CASCADE NATURAL GAS CORPORATION

IGI Resources

Cascade Natural Gas

Eastport, Idaho

231

Sumas, Washington

IGI Resources

Cascade Natural Gas

CASCADE NATURAL GAS CORPORATION

Inland Pacific

Cascade Natural Gas

Sumas, Wastington

CASCADE NATURAL GAS CORPORATION

Mobil Natural Gas inc.

Cascade Natural Gas

Sumas, Washington

$856 \quad 7.64$

509

6.19

$\begin{array}{ll}715 & 2.98\end{array}$

2080

5.68

CASCADE NATURAL GAS CORPORATION

Natural Gas Clearinghouse

Cascade Natural Gas

Sumas, Washington

CASCADE NATURAL GAS CORPORATION

Westcoast Gas Services Inc.

Cascade Natural Gas

Sumas, Washington

Champlain, NY

$42 \quad 2.69$

$38 \quad 2.81$

$44 \quad 2.66$

124

2.72

CHEVRON U.S.A. INC.

Chevron Canada Resources

Chevron U.S.A. Inc.
3036

2.32
2689

2.31

2.34

8797

2.32 
SHORT-TERM SALES

YEAR: 1995 Quarter: One

Estimated Volumes (MMCF) \& Prices (\$ / MMBTU)
Importer

Seller

Purchaser / End User

CIBOLA CORPORATION

Cibola Canada Energy Marketing Co.

Cibola Corporation

CIBOLA CORPORATION

Cibola Canada Energy Marketing Co.

Cook Inlet

CIBOLA CORPORATION

Cibola Canada Energy Marketing Co.

Pacific Gas Transmission

Point
of
Entry

Port of Morgan, MT

Eastport, Idaho

Eastport, Idaho

Eastport, Idaho

Eastport, ldaho

Grand Island, NY

St. Clair, M!

AlG Trading Corporation

Great Lakes Gas Transmission Co.

COASTAL GAS MARKETING COMPANY

AlG Trading Compration

iroquois Gas Transmission

COASTAL GAS MARKETING COMPANY

AIG Trading Corporation

Tennessee Gas Pipeline Company

Niagara Falls, NY

COASTAL GAS MARKETING COMPANY

AlG Trading Corporation

Viking Gas Transmission

Noyes, Minnesota

COASTAL GAS MARKETING COMPANY ANR Pipeline

Coastal Gas Marketing Company

Noyes, Minnesota
- N/A

122

1.56

$0 \quad$ N/A

122

1.56

$0 \quad N / A$

$6 \quad 1,18$

- N/A

$6 \quad 1.18$

January
Vol. Price

Total

Vol.

Price

$19 \quad 0.87$

$\begin{array}{ll}398 & 0.83\end{array}$

$63 \quad 0.91$

$\begin{array}{ll}726 & 1.47\end{array}$

$80 \quad 1.43$

$16 \quad 1.44$

$89 \quad 1.53$

- N/A

- N/A

589

1.53 
SHORT-TERM SALES

YEAR: 1995 Quarter: One

Estimated Volumes (MMCF) \& Prices (\$ / MMBTU)
Importer

Seller

Purchaser / End User

COASTAL GAS MARKETING COMPANY ANR Pipeline

Great Lakes Gas Transmission Co.

Noyes, Minnesota

- N/A

$547 \quad 2.44$

$17 \quad 1.14$

564

2.40

COASTAL GAS MARKETING COMPANY ANR Pipeline

Michigan Gas Utilities

Noyes, Minnesota

$15 \quad 1.54$

$0 \quad$ N/A

O N/A

$15 \quad 1.54$

COASTAL GAS MARKETING COMPANY

Enron Capital \& Trade

Empire State Pipeline

COASTAL GAS MARKETING COMPANY

Gaz Metropolitan, Inc.

Iroquois Gas Transmission

Waddington, NY

- N/A

155

1.57

$0 \quad$ N/A

155

1.57

COASTAL GAS MARKETING COMPANY Inland Pacific

IGI Resources

COASTAL GAS MARKETING COMPANY Inland Pacific

Northwest Pipeline

Sumas, Washington

- N/A

$234 \quad 1.05$

$5 \quad 1.03$

238

1.05

COASTAL GAS MARKETING COMPANY Mobil Natural Gas Canada

Northwest Pipeline

Sumas, Washington

$19 \quad 1.12$

- N/A

$0 \quad$ N/A

$19 \quad 1.12$

COASTAL GAS MARKETING COMPANY North Canadian Marketing Inc.

Coastal Gas Marketing Company

COASTAL GAS MARKETING COMPANY North Canadian Marketing Inc.

Great Lakes Gas Transmission Co.

Noyes, Minnesota

$0 \quad$ N/A

143

1.18

$0 \quad$ N/A

143

1.18

COASTAL GAS MARKETING COMPANY Northridge Gas Marketing

Coastal Gas Marketing Company

Noyes, Minnesota

159

1.36

O N/A

O N/A

159

1.36

COASTAL GAS MARKETING COMPANY

Northridge Gas Marketing

Viking Gas Transmission
Noyes, Minnesota
121

2.36

- N/A

121

2.36 
SHORT-TERM SALES

YEAR: 1995 Quarter: One

Estimated Volumes (MMCF) \& Prices (\$ / MMBTU)
Importer

Seller

Purchaser / End User

COASTAL GAS MARKETING COMPANY Northridge Petroleum Marketing, inc

Viking Gas Transmission

COASTAL GAS MARKETING COMPANY Numac Energy

Northwest Pipeline

Sumas, Washington

O N/A

$50 \quad 1.02$

$0 \quad$ N/A

$50 \quad 1.02$

COASTAL GAS MARKETING COMPANY Petro-Canada Hydrocarbons Inc.

Pacific Gas Transmission

Eastport, Idaho

O N/A

$14 \quad 0.82$

- N/A

14

0.82

COASTAL GAS MARKETING COMPANY Petro-Canada Resources

Northwest Pipeline

Sumas, Washington

- N/A

- N/A

152

1.05

152

1.05

COASTAL GAS MARKETING COMPANY

Petro-Canada Resources

Pacific Gas Transmission

COASTAL GAS MARKETING COMPANY

Poco Petroleum Ltd.

Great Lakes Gas Transmission Co.

Noyes, Minnesota

- N/A

$21 \quad 1.18$

O N/A

21

1.18

COASTAL GAS MARKETING COMPANY Poco Petroleum Ltd.

Northwest Pipeline

COASTAL GAS MARKETING COMPANY

ProGas Limited

Great Lakes Gas Transmission Co.

Noyes, Minnesota

- N/A

442

2.44

- N/A

442

2.44

COASTAL GAS MARKETING COMPANY

ProGas Limited

Michigan Gas Utilities

Noyes, Minnesota

$490 \quad 3.07$

o N/A

o N/A

490

3.07

COASTAL GAS MARKETING COMPANY ProGas Limited

Viking Gas Transmission

Noyes, Minnesota

o N/A

$0 \quad$ N/A

164

1.14

164

1.14

COASTAL GAS MARKETING COMPANY

SaskEnergy

Coastal Gas Marketing Company

Noyes, Minnesota

$672 \quad 2.72$

- N/A

672

2.72 o N/A

(6)


SHORT-TERM SALES

YEAR: 1995 Quarter: One

Estimated Volumes (MMCF) \& Prices (\$ / MMBTU)
Importer

Seller

Purchaser / End User

COASTAL GAS MARKETING COMPANY SaskEnergy

Empire State Pipeline

Grand Island, NY

Noyes, Minnesota

$0 \quad$ N/A

758

2.40

159

1.05

917

2.17

COASTAL GAS MARKETING COMPANY SaskEnergy

Iroquois Gas Transmission

Waddington, NY

$245 \quad 1.78$

$98 \quad 1.56$

$67 \quad 1.58$

410

1.69

COASTAL GAS MARKETING COMPANY

SaskEnergy

Tennessee Gas Pipeline Company

Niagara Falls, NY

$0 \quad$ N/A

$368 \quad 1.55$

O N/A

$368 \quad 1.55$

COASTAL GAS MARKETING COMPANY SaskEnergy

Viking Gas Transmission

Noyes, Minnesota

$0 \quad$ N/A

$137 \quad 1.05$

$59 \quad 1.08$

$196 \quad 1.06$

COASTAL GAS MARKETING COMPANY Selkirk Cogeneration Partners, L.P.

Iroquois Gas Transmission

Waddington, NY

- N/A

$10 \quad 1.56$

$18 \quad 1.56$

$28 \quad 1.56$

COASTAL GAS MARKETING COMPANY Shell Canada Limited

Empire State Pipeline

COASTAL GAS MARKETING COMPANY

Shell Canada Limited

Iroquois Gas Transmission

Waddington, NY

$19 \quad 3.28$

$4 \quad 1.56$

$4 \quad 1.55$

27

2.80

COASTAL GAS MARKETING COMPANY Shell Canada Limited

Tennessee Gas Pipeline Company

Niagara Falls, NY

$5 \quad 1.55$

- N/A

- N/A

$5 \quad 1.55$

COASTAL GAS MARKETING COMPANY Tarpon Gas Marketing Ltd.

Empire State Pipeline

Grand Island, NY

27

1.80

$2 \quad 1.55$

- N/A

29

1.78

COASTAL GAS MARKETING COMPANY Tarpon Gas Marketing Ltd.

Tennessee Gas Pipeline Company
Niagara Falls, NY
$11 \quad 1.55$

- N/A

11

1.55
O N/A 


\section{SHORT-TERM SALES \\ YEAR: 1995 Quarter: One \\ Estimated Volumes (MMCF) \& Prices (\$ / MMBTU)}

Importer

Seller

Purchaser / End User

$$
\begin{gathered}
\begin{array}{c}
\text { Point } \\
\text { of } \\
\text { Entry }
\end{array} \\
\hline
\end{gathered}
$$

COASTAL GAS MARKETING COMPANY

Wascana Energy Marketing Inc.

Pacific Gas Transmission

COENERGY TRADING COMPANY

CoEnergy Trading Company

Central Hudson Gas \& Electric

COENERGY TRADING COMPANY

CoEnergy Trading Company

Empire Natural Gas Corporation

COENERGY TRADING COMPANY

CoEnergy Trading Company

Long Island Lighting Company

COENERGY TRADING COMPANY CoEnergy Trading Company Northeast Utilities

Waddington, NY

- N/A

$0 \quad 1.45$

$0 \quad N / A$

$0 \quad 1.45$

COENERGY TRADING COMPANY Continental Energy Marketing Ltd.

Central Hudson Gas \& Electroc

Waddington, NY

$14 \quad 1.62$

- N/A

$0 \quad$ N/A

$14 \quad 1.62$

COENERGY TRADING COMPANY Continental Energy Marketing Ltd.

Long Island Lighting Company

COENERGY TRADING COMPANY Continental Energy Marketing Ltd. Long Island Lighting Company

Waddington, NY

- N/A

22

1.55

- N/A

22

1.55

COENERGY TRADING COMPANY Continental Energy Marketing Ltd. Northeast Utilities

Niagara Falls, NY

- N/A

267

$0 \quad N / A$

267

1.47

COENERGY TRADING COMPANY Continental Energy Marketing Ltd. Orange \& Rockland Marketing

Niagara Falls, NY

$0 \quad$ N/A

$0 \quad$ N/A

33

1.76

$33 \quad 1.76$

COENERGY TRADING COMPANY

Renaissance Energy Ltd.

Bay State Gas Company
Niagara Falls, NY o N/A

$4 \quad 1.56$

$31 \quad 1.67$

$34 \quad 1.66$ 
SHORT-TERM SALES

YEAR: 1995 Quarter: One

Estimated Volumes (MMCF) \& Prices (\$ / MMBTU)
Importer

Seller

Purchaser / End User

COENERGY TRADING COMPANY Renaissance Energy Ltd.

Bay State Gas Compariy

COENERGY TRADING COMPANY Selkirk Cogeneration Partners, L.P. Long Island Lighting Company

CONSUMERS POWEFI COMPANY Husky Oil

Consumers Power Company

Point

Entry

Waddington, NY

Waddington, NY

$10 \quad 1.57$

- N/A

- N/A

February

Vol. Price

Vol. Price

March

Vol. Price

Quarterly

Total

Avg

Vol. Price

Noyes, Minnesota

465

2.10

420

2.10

465

2.10

1350

2.10

CONSUMERS POWEFI COMPANY

Norcen Energy Resourses

Consumers Power Company

Noyes, Minnesota

434

2.10

39

2.10

434

2.10

1260

2.10

CONSUMERS POWEFI COMPANY

North Canadian Oils Lirnited

Consumers Power Company

Noyes, Minnesota

310

2.10

280

2.10

310

2.10

900

2.10

CONSUMERS POWEF COMPANY

Poco Petroleum Ltd.

Consumers Power Company

Noyes, Minnesota

465

2.13

420

2.13

465

2.13

1350

2.13

CONSUMERS POWEFI COMPANY

Shell Canada Limited

Consumers Power Company

Noyes, Minnesota

349

2.12

315

2.12

349

2.12

1013

2.12

CONSUMERS POWEF COMPANY

Western Gas Marketing Limited
Consumers Power Company

Noyes, Minnesota

CONTINENTAL ENERGY MARKETING LTD.

Continental Energy Marketing Ltd.

Barretts Minerals

Massena, New York

CONTINENTAL ENERGY MARKETING LTD.

Continental Energy Marketing Ltd.

Canton Central School

Massena, New York

CONTINENTAL ENERGY MARKETING LTD.

Continental Energy Marketing Ltd.

Clarkson University

Massena, New York
$349 \quad 2.09$

31

2.09

$330 \quad 2.09$

994

2.09

$6 \quad 2.52$

$5 \quad 2.52$

$6 \quad 2.52$

$16 \quad 2.52$

$2 \quad 2.52$

$1 \quad 2.52$

$2 \quad 2.52$

$4 \quad 2.52$

$11 \quad 5.04$

$10 \quad 5.04$

$11 \quad 5.04$

33

5.04 
Page -89

SHORT-TERM SALES

YEAR: 1995 Quarter: One

Estimated Volumes (MMCF) \& Prices (\$ / MMBTU)
Importer

Seller

Purchaser / End User
Point

of

Entry
January
Vol. Price

$0 \quad N / A$

328

February

Vol. Price
Quarterly

Total

Vol. ${ }^{\text {Avg. }}$ Price

CONTINENTAL ENERGY MARKETING LTD. Continental Energy Marketing Ltd.

CoEnergy Trading Company

Massena, New York

$0 \quad N / A$

328

CONTINENTAL ENERGY MARKETING LTD.

Continental Energy Marketing Ltd.

CoEnergy Trading Company

Niagara Falls, NY

- N/A

- N/A

$33 \quad 1.76$

33

1.76

CONTINENTAL ENERGY MARKETING LTD.

Continental Energy Marketing Ltd.

CoEnergy Trading Company

Waddington, NY

- N/A

$22 \quad 1.55$

165

1.56

187

1.56

CONTINENTAL ENERGY MARKETING LTD.

Continental Energy Marketing Ltd.

College of Technology

Massena, New York

$7 \quad 2.52$

$6 \quad 2.52$

$7 \quad 2.52$

$21 \quad 2.52$

CONTINENTAL ENERGY MARKETING LTD.

Continental Energy Marketing Ltd.

Continental Energy Marketing Ltd.

Eastport, Idaho

69

1.10

O N/A

O N/A

$69 \quad 1.10$

CONTINENTAL ENERGY MARKETING LTD.

Continental Energy Marketing Ltd.

Continental Energy Marketing Ltd.

Waddington, NY

$14 \quad 1.68$

o N/A

- N/A

$14 \quad 1.68$

CONTINENTAL ENERGY MARKETING LTD.

Continental Energy Marketing Ltd.

Comell Linen

Massena, New York

$2 \quad 2.52$

$2 \quad 2.52$

$2 \quad 2.52$

$6 \quad 2.52$

CONTINENTAL ENERGY MARKETING LTD.

Continental Energy Marketing Ltd.

Flanders inn

Massena, New York

$0 \quad 2.52$

$0 \quad 2.52$

$0 \quad 2.52$

1

2.52

CONTINENTAL ENERGY MARKETING LTD.

Continental Energy Marketing Ltd.

Heuvelton Central School

Massena, New York

1252

$1 \quad 2.52$

$1 \quad 2.52$

$3 \quad 2.52$

CONTINENTAL ENERGY MARKETING LTD.

Continental Energy Marketing Ltd.

Highland Nursing Home

Massena, New York

$0 \quad 2.52$

$0 \quad 2.52$

$0 \quad 2.52$

$1 \quad 2.52$

CONTINENTAL ENERGY MARKETING LTD.

Continental Energy Marketing Ltd.

Howard Energy Company

Eastport, Idaho

$54 \quad 0.84$

$0 \quad$ N/A

54

0.84 


\section{SHORT-TERM SALES \\ YEAR: 1995 Quarter: One}

Estimated Volumes (MMCF) \& Prices (\$ / MMBTU)

Importer

Seller

Purchaser / End User
Point

Entry
January

Vol. Price
February
Vol. Price
Quarterly Total

March

Vol. Price
Avg.

Vol Price

CONTINENTAL ENERGYY MARKETING LTD.

Continental Energy Marketing Ltd.

Howard Energy Company

CONTINENTAL ENERGY MARKETING LTD.

Continental Energy Marketing Ltd.

Lisbon Central School

CONTINENTAL ENERGY MARKETING LTD.

Continental Energy Marketing Ltd.

Madrid-Waddington School

CONTINENTAL ENERGYY MARKETING LTD.

Continental Energy Marketing Ltd.

Massena Central School

CONTINENTAL ENERGY MARKETING LTD.

Continental Energy Marketing Ltd.

Massena Memorial Hospital

CONTINENTAL ENERGY MARKETING LTD.

Continental Energy Marketing Ltd.

Northridge Gas Marketing Inc.

CONTINENTAL ENERGY MARKETING LTD.

Continental Energy Marketing Ltd.

Ogdensboro

CONTINENTAL ENERGY MARKETING LTD.

Continental Energy Marketing Ltd.

Potsdam Central School

Massena, New York

$2 \quad 2.52$

12.52

$2 \quad 2.52$

5

CONTINENTAL ENERGY MARKETING LTD.

Continental Energy Ma keting Ltd.

St. Lawrence University

Massena, New York

O N/A

- N/A

$137 \quad 1.63$

137

1.63

CONTINENTAL ENERGY MARKETING LTD.

Continental Energy Makketing Ltd.

St. Regis Nursing Home

Massena, New York

$0 \quad 2.52$

$0 \quad 2.52$

$0 \quad 2.52$

$1 \quad 2.52$

CONTINENTAL ENERGY MARKETING LTD.

Continental Energy Marketing Ltd.

Texas Ohio, West Inc.

Eastport, Idaho

$0 \quad$ N/A

$83 \quad 1.16$

$0 \quad$ N/A

83

1.16 
SHORT-TERM SALES

YEAR: 1995 Quarter: One

Estimated Volumes (MMCF) \& Prices (\$ / MMBTU)
Importer

Seller

Purchaser / End User

$$
\text { Point }
$$

Entry
January
Vol. Price
Eastport, Idaho

Continental Energy Marketing Ltd.

Texas-Ohio Gas, Inc.

CONWEST EXPLORATION COMPANY LIMITED

Conwest Exploration Company Limited

Enron Gas Marketing

Port of Morgan, MT

CRESTAR ENERGY MARKETING CORPORATION

Crestar Energy

Cibola Corporation

Port of Morgan, MT

CRESTAR ENERGY MARKETING CORPORATION

Crestar Energy

Northem States Power (MN)

Port of Morgan, MT

Eastport, Idaho

Eastport, Idaho

CU ENERGY MARKETING, INC

Continental Energy Marketing Lid.

Sunrise Energy

CU ENERGY MARKETING, INC.

Maple View Gas Marketing

Sunrise Energy

Eastport, Idaho

$61 \quad 1.74$

$0 \quad$ N/A

o N/A

$61 \quad 1.74$

DARTMOUTH POWER ASSOCIATES L.P.

Brymore Energy, Ltd.

Dartmouth Power Associates

Waddington, NY

$42 \quad 2.21$

$21 \quad 2.30$

$54 \quad 2.07$

117

2.16

DARTMOUTH POWER ASSOCIATES L.P.

Dartmouth Power Associates

DGS Trading.

Waddington, NY

$0 \quad$ N/A

- N/A

$1 \quad 0.89$

$1 \quad 0.89$

DARTMOUTH POWER ASSOCIATES L.P. Dartmouth Power Associates JMC Fuel Services

Waddington, NY

- N/A

10.84

- N/A

$1 \quad 0.84$

DARTMOUTH POWER ASSOCIATES L.P. Dartmouth Power Associates

Pawtucket Power Associates
Waddington, NY
$89 \quad 1.17$

$54 \quad 1.01$

$552 \quad 1.35$

$432 \quad 1.01$

20.99

Quarterly
Total

Avg.

Vol. Price 
SHORT-TERM SALES

YEAR: 1995 Quarter: One

Estimated Volumes (MMCF) \& Prices (\$/MMBTU)
Importer

Seller

Purchaser / End User

$$
\begin{aligned}
& \text { Point } \\
& \text { of } \\
& \text { Entry }
\end{aligned}
$$

Waddington, NY

Waddington, NY

Dartmouth Power Associates

Yankee Gas Services

DEKALB ENERGY COMPANY

Dekalb Energy Canada Ltd.

Enron Capital \& Trade Resources

DEKALB ENERGY COMPANY

Dekalb Energy Canada Ltd.

Gaylord Container Corporation

DEKALB ENERGY COMPANY

Dekalb Energy Canada Ltd.

Redwood Resources inc.

DGS TRADING INC

Canadian Hunter Marketing Ltd.

Bay State Gas Company

DGS TRADING INC.

Canadian Hunter Marketing Ltd.

Boston Edison Company

DGS TRADING INC.

Cowest Energy

Boston Edison Company

DGS TRADING INC.

Enron Gas Marketing Canada

Bay State Gas Compary

DGS TRADING INC

Enron Gas Marketing Canada

Boston Edison Company

DGS TRADING INC.

Louis Dreyfus Energy Corporation

Boston Edison Company

Eastport, Idaho

Eastport, Idaho

Eastport, Idaho
January
Vol. February
Price

March

Vol. Price

Quarterly

Total

Avg.

Vol. Price

- N/A

$0 \quad$ N/A

$34 \quad 0.69$

34

0.69

$319 \quad 0.93$

265

0.72

$289 \quad 0.69$

874

0.79

- N/A

22

0.72

- N/A

22

0.72

Grand island, NY

- N/A

$\begin{array}{ll}78 & 1.82\end{array}$

- N/A

$\begin{array}{ll}78 & 1.82\end{array}$

Grand Island, NY

$\begin{array}{ll}65 & 1.58\end{array}$

- N/A

- N/A

$65 \quad 1.58$

Grand Island, NY

$131 \quad 1.59$

- N/A

128

1.61

259

1.60

Grand Island, NY

- N/A

155

2.12

- N/A

155

2.12

Grand Island, NY

140

2.12

- N/A

155

1.94

295

2.03

Grand Isiand, NY
- N/A

$60 \quad 2.42$

$31 \quad 2.42$

$91 \quad 2.42$ 
SHORT-TERM SALES

YEAR: 1995 Quarter: One

Estimated Volumes (MMCF) \& Prices (\$/MMBTU) importer

Seller

Purchaser/End User

DGS TRADING INC.

Louis Dreyfus Energy Corporation Central Hudson Gas \& Electric

DGS TRADING INC.

Phibro Energy

Boston Edison Company

DGS TRADING INC.

Phibro Energy

New England Power Company

DGS TRADING INC.

ProGas Limited

Bay State Gas Company

DGS TRADING INC.

ProGas Limited

Boston Edison Company

DGS TRADING INC.

Renaissance Energy Ltd.

Bay State Gas Company

DGS TRADING INC.

Renaissance Energy Ltd.

Connecticut Power \& Light

DGS TRADING INC.

Stamp Gas Inc.

Bay State Gas Company

DGS TRADING INC.

Stamp Gas inc.

Pawtucket Power Associates

Grand Island, NY

- N/A

- N/A

$56 \quad 1.59$

$56 \quad 1.59$

DGS TRADING INC.

Wascana Energy Marketing Inc.

Connecticut Power \& Light

DGS TRADING INC.

Wascana Energy Marketing Inc.

Pawtucket Power Associates

Point

Entry

Grand Island, NY

Grand Island, NY

210

- N/A

$50 \quad 1.64$

$0 \quad$ N/A

$50 \quad 1.64$

Grand Island, NY

o N/A

- N/A

$30 \quad 1.58$

$30 \quad 1.58$

Grand Island, NY

$\begin{array}{llll}0 & \text { N/A } & 395 & 1.77\end{array}$

$0 \quad$ N/A

$395 \quad 1.77$

Grand Island, NY

$15 \quad 1.52$

- N/A

$35 \quad 1,62$

$50 \quad 1.59$

Grand Island, NY

- N/A

$42 \quad 1.81$

- N/A

42

1.81

Grand Island, NY

$84 \quad 1.64$

- N/A

- N/A

$84 \quad 1.64$

Grand Island, NY
- N/A

96

1.79

$93 \quad 1.65$

189

1.72 
Importer

Seller

Purchaser / End User

DIRECT ENERGY MAFIKETING LIMITED

Direct Energy Marketing Limited

AIG Trading Corporation

DIRECT ENERGY MAFIKETING LIMITED

Direct Energy Marketing Limited

Chevron U.S.A. Inc.

Eastport, Idaho

$114 \quad 3.07$

122

February

Vol. Price

Vol. Price

March

Vol. Price

Quarterly

Total

vol. Price

Noyes, Minnesota

$1 \quad 1.17$

- N/A

O N/A

1

1.17

DIRECT ENERGY MAFIKETING LIMITED

Direct Energy Marketing Limited

Coastal Gas Marketing Company

DIRECT ENERGY MARKETING LIMITED

Direct Energy Marketing Limited

Howard Energy Company

Eastport, Idaho

O N/A

$83 \quad 1.05$

155

1.05

237

1.05

DIRECT ENERGY MAFKETING LIMITED

Direct Energy Marketing Limited

$M$ \& $B$ Industrial Gas Development

Niagara Falls, NY

$30 \quad 1.91$

$27 \quad 1.69$

- N/A

$57 \quad 1.81$

DIRECT ENERGY MARKETING LIMITED

Direct Energy Marketing Limited

Meridian Marketing

Grand Island, NY

$0 \quad$ N/A

$65 \quad 1.62$

$3 \quad 1.75$

68

1.63

DIRECT ENERGY MAFIKETING LIMITED

Direct Energy Marketing Limited

Mock Resources

Eastport, Idaho

- N/A

$217 \quad 1.43$

$3 \quad 1.12$

221

1.43

DIRECT ENERGY MAFIKETING LIMITED

Direct Energy Marketing Limited

National Gas Resources

Eastport, Idaho

$30 \quad 1.99$

$205 \quad 2.97$

144

3.23

379

2.99

DIRECT ENERGY MARIKETING LIMITED

Direct Energy Marketing Limited

North Coast Energy, Inc.

Niagara Falls, NY

$\begin{array}{ll}73 & 1.91\end{array}$

$58 \quad 1.67$

$12 \quad 1.70$

144

1.79

DIRECT ENERGY MARKETING LIMITED Direct Energy Marketinc Limited

Northeast Heat \& Light

Grand Island, NY

$0 \quad$ N/A

O N/A

$18 \quad 1.67$

$18 \quad 1.67$

DIRECT ENERGY MARKETING LIMITED

Direct Energy Marketing Limited

Westcoast Gas Services; (U.S.A.) Inc
Noyes, Minnesota
$0 \quad N / A$

O N/A

26

1.68 


\section{SHORT-TERM SALES \\ YEAR: 1995 Quarter: One \\ Estimated Volumes (MMCF) \& Prices (\$ / MMBTU)}

Importer

Seller

Purchaser/End User

ENRON GAS MARKETING, INC.

AIG Trading Corporation

Enron Gas Marketing

ENRON GAS MARKETING, INC

British Columbia Gas Utility

Enron Gas Marketing

Point

Entry

Sumas, Washington

Sumas, Washington

Sumas, Washington

CanWest Gas Supply inc.

Enron Gas Marketing

ENRON GAS MARKETING, INC.

Conwest Exploration Co. Ltd.

Enron Gas Marketing

Eastport, ldaho

Sumas, Washington

$40 \quad 1.05$

$20 \quad 0.99$

279

2.02

339

1.84

ENRON GAS MARKETING, INC. Enron Gas Marketing Canada Centra Gas

Noyes, Minnesota

$134 \quad 1.61$

121

1.30

133

1.41

388

1.44

ENRON GAS MARKETING, INC. Enron Gas Marketing Canada Centra Gas

Port of Morgan, MT

$520 \quad 1.22$

ENRON GAS MARKETING, INC. Enron Gas Marketing Canada

Enron Gas Marketing

Eastport, Idaho

ENRON GAS MARKETING, INC.

Enron Gas Marketing Canada

Enron Gas Marketing

Grand Island, NY

$155 \quad 2.12$

141

2.12

155

1.93

451

2.05

ENRON GAS MARKETING, INC.

Enron Gas Marketing Canada

Enron Gas Marketing

Niagara Falls, NY

887

2.10

$617 \quad 2.02$

719

1.68

2223

1.94

ENRON GAS MARKETING, INC.

Enron Gas Marketing Canada

Enron Gas Marketing
Sumas, Washington
$519 \quad 1.06$

420 


\section{SHORT-TERM SALES \\ YEAR: 1995 Quarter: One \\ Estimated Volumes (MMCF) \& Prices (\$ / MMBTU)}

Importer

Seller

Purchaser / End User

ENRON GAS MARKETING, INC

Enron Gas Marketing Canada

Enron Gas Marketing

ENRON GAS MARKETING, INC.

Enron Gas Marketing Cianada

The Montana Power Company

ENRON GAS MARKETING, INC.

Petro Source Gas Ventures

Enron Gas Marketing

Eastport, Idaho

$42 \quad 1.96$

O N/A

$0 \quad$ N/A

$42 \quad 1.96$

ENRON GAS MARKETING, INC.

Selkirk Cogeneration

Enron Gas Marketing

Niagara Falls, NY

$20 \quad 1.76$

$0 \quad$ N/A

- N/A

$20 \quad 1.76$

ENRON GAS MARKETING, INC

Westcoast Gas Services Inc.

Enron Gas Marketing

Sumas, Washington

$3 \quad 0.94$

- N/A

51.00

$8 \quad 0.98$

EQUITABLE RESOURCES MARKETING COMPANY

Equitable Resources Marketing Canad

Equitable Resources Marketing Compa

Eastport, Idaho

508

4.32

356

7.97

882

5.77

GAZ METROPOLITAIN AND COMPANY, L.P.

Gaz Metropolitain, Inc.

JMC Fuel Services

Waddington, NY

$93 \quad 1.17$

$84 \quad 0.98$

$0 \quad$ N/A

177

1.08

GAZ METROPOLITAIN AND COMPANY, L.P.

Gaz Metropolitain, Inc.

Long Island Lighting Company

Waddington, NY

310

1.25

$280 \quad 1.06$

$310 \quad 1.42$

900

GAZ METROPOLITAIN AND COMPANY, L.P.

Gaz Metropolitain, Inc.

Transco Energy Marketing Company Waddington, NY

1.25

$132 \quad 1.52$

$0 \quad$ N/A

352

1.35

GAZ METROPOLITAIN AND COMPANY, L.P.

Gaz Metropolitain, Inc.

United City

Waddington, NY

$90 \quad 1.80$

$148 \quad 1.80$

O N/A

238

1.80

GREAT FALLS GAS COMPANY

Coastal Gas Marketing, Canada

Great Falls Gas Company

(SP)

Babb, Montana

$\begin{array}{ll}82 & 1.78\end{array}$

117

1.78

289

1.78

SP Internuptible/special purchase sale made under Long-term authorization. 


\section{SHORT-TERM SALES \\ YEAR: 1995 Quarter: One \\ Estimated Volumes (MMCF) \& Prices (\$/MMBTU)}

Importer
Seller

Purchaser / End User

GREAT FALLS GAS COMPANY (SP)

Eagle Gas Marketing

Great Falls Gas Company

HUSKY GAS MARKETING, INC.

Husky Oil

AmGas Inc.

HUSKY GAS MARKETING, INC.

Husky Oil

Arcadian Corporation

HUSKY GAS MARKETING, INC.

Husky Oil

Cibola Corporation

HUSKY GAS MARKETING, INC.

Husky Oil

Coastal Gas Marketing Company

HUSKY GAS MARKETING, INC.

Husky Oil

Consumers Power Company

HUSKY GAS MARKETING, INC.

Husky Oil

Enron Gas Marketing

Port of Morgan, MT

$388 \quad 1.00$

$214 \quad 0.91$

130

0.90

732

0.96

HUSKY GAS MARKETING, INC.

Husky Oil

Enron Gas Marketing

Sumas, Washington

$0 \quad$ N/A

101

1.56

- N/A

10

1.56

HUSKY GAS MARKETING, INC.

Husky Oil
Equitable Resources Marketing Compa

Port of Morgan, MT

o N/A

$0 \quad$ N/A

$5 \quad 0.90$

$5 \quad 0.90$

HUSKY GAS MARKETING, INC.

Husky Oil

Grand Valley Gas Company

Port of Morgan, MT

- N/A

D N/A

151

0.90

151

0.90

HUSKY GAS MARKETING, INC.

Husky Oil

IGI Resources

Sumas, Washington $\begin{array}{llll}0 & \text { N/A } & 161 & 1.56\end{array}$

13.04

163

1.57

SP Interruptible/special purchase sale made under Long-term authorization. 
SHORT-TERM SALES

YEAR: 1995 Quarter: One

Estimated Volumes (MMCF) \& Prices (\$ / MMBTU)
Importer

Seller

Purchaser / End User

HUSKY GAS MARKETING, INC.

Husky Oil

Interenergy Corporation

HUSKY GAS MARKETING, INC. Husky Oil

Koch Gas Services Company

HUSKY GAS MARKETING, INC.

Husky Oil

Koch Gas Services Company

HUSKY GAS MARKETING, INC.

Husky Oil

Midcon Gas Services Corporation

HUSKY GAS MARKETING, INC.

Husky Oil

Midwest Gas Company

HUSKY GAS MARKETING, INC. Husky Oil

Mock Resources

HUSKY GAS MARKETING, INC.

Husky Oil

National Gas Resources

HUSKY GAS MARKETING, INC

Husky Oil

Natural Gas Clearinghouse

HUSKY GAS MARKETING, INC

Husky Oil

NorAm Energy Services

Port of Morgan, MT

$29 \quad 0.91$

$41 \quad 0.90$

209

0.97

HUSKY GAS MARKETING, INC. Husky Oil

Novagas Clearinghousse

Sumas, Washington

o N/A

$0 \quad N / A$

$11 \quad 3.04$

11

3.04

HUSKY GAS MARKETING, INC.

Husky Oil

Omaha Utilities District
Port of Morgan, MT
Port of Morgan, MT

January

Vol. Price

March

vol. Price

Total

Avg.

Vol. Price

Port of Morgan, MT

$166 \quad 1.00$

273

589

0.93

Port of Morgan, MT

$10 \quad 1.00$

- N/A

$0 \quad N / A$

10

1.00

Sumas, Washington

$35 \quad 1.67$

$0 \quad$ N/A

$35 \quad 1.67$

Port of Morgan, MT

$135 \quad 1.00$

- N/A

$0 \quad N / A$

135

1.00

$6 \quad 3.04$

Sumas, Washington
$21 \quad 1.00$

$0 \quad N / A$

$0 \quad$ N/A

$21 \quad 1.00$ 


\section{SHORT-TERM SALES \\ YEAR: 1995 Quarter: One \\ Estimated Volumes (MMCF) \& Prices (\$ / MMBTU)}

Importer

Seller

Purchaser / End User

HUSKY GAS MARKETING, INC. Husky Oil

Peoples Natural Gas Company

HUSKY GAS MARKETING, INC. Husky Oil

Southwest Gas Corporation

HUSKY GAS MARKETING, INC. Husky Oil

Tenaska Marketing Ventúres

HUSKY GAS MARKETING, INC. Husky Oil

Tenaska Marketing Ventures

HUSKY GAS MARKETING, INC. Husky Oil

U.S. Gas Transportation Inc.

HUSKY GAS MARKETING, INC.

Husky Oil

Washington Natural Gas Company

IGI RESOURCES INC.

AEC Oil \& Gas Company

Boise Cascade Corporation

IGI RESOURCES INC

AEC Oil \& Gas Company

California Industrial End-users

IGI RESOURCES INC.

AEC Oil \& Gas Company

Cascade Natural Gas

IGI RESOURCES INC.

AEC Oil \& Gas Company

Chevron Chemical

Eastport, Idaho

Port of Morgan, MT

o N/A

o N/A

215

0.90

$215 \quad 0.90$

Sumas, Washington

- N/A

$41 \quad 1.51$

- N/A

$41 \quad 1.51$

Port of Morgan, MT

$451 \quad 0.91$

$466 \quad 0.90$

$1472 \quad 0.94$

Sumas, Washington

$45 \quad 1.67$

- N/A

o N/A

$45 \quad 1.67$

Port of Morgan, MT

$155 \quad 1.00$

58

0.91

$0 \quad N / A$

214

0.98

Sumas, Washington

$70 \quad 1.67$

$447 \quad 2.68$

$82 \quad 3.04$

600

Eastport, Idaho

- N/A

- N/A

$0 \quad 1.66$

$0 \quad 1.66$

Eastport, Idaho

- N/A

- N/A

$0 \quad 0.83$

$0 \quad 0.83$

Eastport, Idaho

$0 \quad N / A$

- N/A

10.83

$1 \quad 0.83$

IGI RESOURCES INC.

AEC Oil \& Gas Company

Grand Valley Gas Company
Eastport, Idaho
$0 \quad N / A$

- N/A

$4 \quad 0.83$

4 


\section{SHORT-TERM SALES \\ YEAR: 1995 Quarter: One \\ Estimated Volumes (MMCF) \& Prices (\$ / MMBTU)}

importer

Seller

Purchaser / End User

IGI RESOURCES INC.

AEC Oil \& Gas Company

Lamb Weston

IGI RESOURCES INC.

AEC Oil \& Gas Compariy

PACCO

IGI RESOURCES INC.

AEC Oil \& Gas Comparyy

Pacific Gas \& Electric Company

IGI RESOURCES INC.

AEC Oil \& Gas Company

Sierra Pacific Power Company

IGI RESOURCES INC.

AEC Oil \& Gas Company

WP Natural Gas

Eastport, Idaho

Eastport, Idaho

RCESINC.

AEC Oil \& Gas Company

Weyemauser

IGI RESOURCES INC.

Amoco Canada Petroleum Company Ltd. Boise Cascade Corporation

Eastport, Idaho

IGI RESOURCES INC

Amoco Canada Petrolesum Company Ltd. Califomia Industrial End-users

Eastport, Idaho

IG] RESOURCES INC

Amoco Canada Petroleum Company Ltd. Cascade Natural Gas

IGI RESOURCES INC.

Amoco Canada Petroleum Company Ltd. Chevron Chemical

Eastport, Idaho

26

2.78

27

2.34

47

3.10

100

2.81

IGI RESOURCES INC.

Amoco Canada Petroleum Company Ltd. Grand Valley Gas Company

Vol. Price

February

Vol. Price

March

vol. Price

Quartenly

Total

Vol. Price

- N/A

- N/A

$0 \quad 0.83$

$0 \quad 0.83$

- N/A

$0 \quad$ N/A

10.83

$1 \quad 0.83$

- N/A

- N/A

$\begin{array}{ll}0 & 0.83\end{array}$

0

0.83

Eastport, Idaho

2.78

19

2.34

31

3.10

65

2.80

Eastport, Idaho
39

2.34

85

3.10

197

2.83 


\section{SHORT-TERM SALES \\ YEAR: 1995 Quarter: One \\ Estimated Volumes (MMCF) \& Prices (\$ / MMBTU)}

Importer

Seller

Purchaser / End User

IGI RESOURCES INC.

Amoco Canada Petroleum Company Ltd. J.R. Simplot Company

IGI RESOURCES INC.

Amoco Canada Petroleum Company Ltd. Lamb Weston

IGI RESOURCES INC.

Amoco Canada Petroleum Company Ltd. PACCO

Eastport, Idaho

IGI RESOURCES INC.

Amoco Canada Petroleum Company Ltd.

Pacific Gas \& Electric Company

Point
of
Entry

Eastport, Idaho

Eastport, Idaho

Eastport, Idaho

IGI RESOURCES INC.

Amoco Canada Petroleum Company Ltd. Portland General Electric Company

IGI RESOURCES INC.

Amoco Canada Petroleum Company Ltd. Sierra Pacific Power Company

Eastport, Idaho

Eastport, Idaho

Amoco Canada Petroleum Company Ltd Universal Frozen Foods

IGI RESOURCES INC.

Amoco Canada Petroleum Company Ltd. WP Natural Gas

Eastport, Idaho

IGI RESOURCES INC.

Amoco Canada Petroleum Company Ltd. Weyerhauser

Eastport, Idaho

Eastport, Idaho

Associated Gas Services, inc.

Boise Cascade Corporation

IGI RESOURCES INC.

Associated Gas Services, inc.

Califomia industrial End-users

Eastport, Idaho

Eastport, Idaho

January
Vol. Price $\quad$ Vol. : Price

March
Vol.

Vol. Price

Quarterly

Total

Vol. ${ }^{\text {Avg. }}$ Price

38

2.78

$47 \quad 2.34$

$0 \quad N / A$

85

- N/A

12.34

$0 \quad 3.10$

$2 \quad 2.46$

$2 \quad 2.78$

$3 \quad 2.34$

o N/A

$5 \quad 2.52$

$\begin{array}{ll}6 & 2.78\end{array}$

$34 \quad 2.34$

34

3.10

$74 \quad 2.72$

26

2.78

$8 \quad 2.34$

54

3.10

88

156

1.39

- N/A

$\begin{array}{ll}7 & 1.28\end{array}$

162

1.39 
SHORT-TERM SALES

YEAR: 1995 Quarter: One

Estimated Volumes (MMCF) \& Prices (\$ / MMBTU)
Importer

Seller

Purchaser / End User

IGI RESOURCES INC.

Associated Gas Services, Inc. Cascade Natural Gas

IGI RESOURCES INC.

Associated Gas Services, Inc.

Chevron Chemical

IGI RESOURCES INC.

Associated Gas Services, Inc. Grand Valley Gas Company

IGI RESOURCES INC.

Associated Gas Services, Inc.

J.R. Simplot Company

IGI RESOURCES INC.

Associated Gas Services, Inc. Lamb Weston

Eastport, Idaho

$15 \quad 1.39$

20

February

Vol. Price

Vol. Price

March

Vol Price

$41 \quad 1.39$

33

1.37

$24 \quad 1.28$

98

1.36

Eastport, Idaho

$68 \quad 1.39$

$47 \quad 1.37$

$37 \quad 1.28$

152

1.36

Eastport, Idaho

$192 \quad 1.39$

$\begin{array}{ll}68 & 1.37\end{array}$

$66 \quad 1.28$

326

IGI RESOURCES INC

Associated Gas Services, inc. PACCO

Eastport, Idaho

- N/A

$0 \quad$ N/A

$0 \quad 1.28$

$0 \quad 1.28$

IGI RESOURCES INC

Associated Gas Services, Inc.

Pacific Gas \& Electric Company

Eastport, Idaho

$52 \quad 1.39$

O N/A

$11 \quad 1.28$

$63 \quad 1.37$

IGI RESOURCES INC

Associated Gas Services, Inc.

Portland General Electric Company

Eastport, Idaho

$99 \quad 1.39$

$82 \quad 1.37$

$0 \quad$ N/A

181

1.38

IGI RESOURCES INC.

Associated Gas Services, Inc.

Sierra Pacific Power Company

Eastport, Idaho

$0 \quad$ N/A

21.37

$0 \quad 1.28$

3

1.36

IGI RESOURCES INC.

Associated Gas Services, Inc.

Universal Frozen Foods

Eastport, Idaho

$6 \quad 1.39$

$5 \quad 1.37$

- N/A

11

1.38

IGI RESOURCES INC

Associated Gas Services, Inc.

WP Natural Gas
Eastport, Idaho
$60 \quad 1.37$

26

1.28

102

1.35 
SHORT-TERM SALES

YEAR: 1995 Quarter: One

Estimated Volumes (MMCF) \& Prices (\$ / MMBTU)
Importer

Seller

Purchaser / End User

IGI RESOURCES INC.

Associated Gas Services, Inc.

Weyerhauser

IGI RESOURCES INC.

CanStates Gas Marketing

Boise Cascade Corporation

IGI RESOURCES INC.

CanStates Gas Marketing

Califomia Industrial End-users

Eastport, Idaho

Eastport, Idaho

January

Vol. Price

February

Vol. Price

March

Entry

Eastport, Idaho

$67 \quad 1.39$

$15 \quad 1.37$

$42 \quad 1.28$

124

1.35

Eastpont, Idaho

- N/A

$0 \quad$ N/A

$0 \quad 1.60$

$0 \quad 1.60$

IGI RESOURCES INC.

CanStates Gas Marketing

Cascade Natural Gas

IGI RESOURCES INC.

CanStates Gas Marketing

Chevron Chemical

Eastport, Idaho

$0 \quad 0.90$

$1 \quad 0.87$

$2 \quad 0.80$

$3 \quad 0.83$

IGI RESOURCES INC.

CanStates Gas Marketing

Grand Valley Gas Company

IGI RESOURCES INC.

CanStates Gas Marketing

J.R. Simplot Company

IGI RESOURCES INC.

CanStates Gas Marketing

Lamb Weston

Eastport, Idaho

$0 \quad 0.90$

0.87

1

0.80

10.83

IGI RESOURCES INC.

CanStates Gas Marketing

Pacific Gas \& Electric Company

Eastport, Idaho

0.90

- N/A

10.80

$1 \quad 0.83$

IGI RESOURCES INC.

CanStates Gas Marketing

Portland General Electric Company

Eastport, Idaho

$1 \quad 0.90$

1

0.87

- N/A

20.88

IGI RESOURCES INC.

CanStates Gas Marketing

Sierra Pacific Power Company
Eastport, Idaho
0.87

$0 \quad 0.80$

0

0.85 
SHORT-TERM SALES

YEAR: 1995 Quarter: One

Estimated Volumes (MMCF) \& Prices (\$ / MMBTU)

Importer

Seller

Purchaser / End User

IGI RESOURCES INC.

CanStates Gas Marketing

Universal Frozen Foods

IGI RESOURCES INC

CanStates Gas Marketing

WP Natural Gas

IGI RESOURCES INC

CanStates Gas Marketing

Weyerhauser

Eastport, Idaho

$0 \quad 0.90$

$0 \quad 0.87$

20.80

$3 \quad 0.82$

IGI RESOURCES INC

CanWest Gas Supply Inc.

Boise Cascade Corporation

IGI RESOURCES INC.

CanWest Gas Supply inc.

Califomia Industrial End-users

Eastport, Idaho

$0 \cdot N / A$

- N/A

0.80

1

IGI RESOURCES INC

CanWest Gas Supply Inc.

Cascade Natural Gas

IGI RESOURCES INC.

CanWest Gas Supply Inc.

Chevron Chemical

Eastport, Idaho

- N/A

- N/A

$3 \quad 0.80$

3

0.80

IGI RESOURCES INC.

CanWest Gas Supply Inc.

Grand Valley Gas Company

IGI RESOURCES INC.

CanWest Gas Supply Inc.

J.R. Simplot Company

IGI RESOURCES INC:

CanWest Gas Supply inc.

Lamb Weston

Eastport, Idaho

o N/A

- N/A

10.80

1

0.80

IGI RESOURCES INC:

CanWest Gas Supply Inc.

PACCO
Eastport, Idaho
$0 \quad$ N/A

0.80

$0 \quad 0.80$ 
SHORT-TERM SALES

YEAR: 1995 Quarter: One

Estimated Volumes (MMCF) \& Prices (\$ / MMBTU)
Importer

Seller

Purchaser / End User

IGI RESOURCES INC.

CanWest Gas Supply inc.

Pacific Gas \& Electric Company

IGI RESOURCES INC.

CanWest Gas Supply Inc.

Sierra Pacific Power Company

IGI RESOURCES INC.

CanWest Gas Supply inc.

WP Natural Gas

IGI RESOURCES INC

CanWest Gas Supply Inc.

WP Natural Gas

IGI RESOURCES INC.

CanWest Gas Supply inc.

Weyerhauser

IGI RESOURCES INC.

Enron Gas Marketing Canada

Boise Cascade Corporation

IGI RESOURCES INC.

Enron Gas Marketing Canada

Califomia Industrial End-users

IGI RESOURCES INC.

Enron Gas Marketing Canada Cascade Natural Gas

IGI RESOURCES INC.

Enron Gas Marketing Canada Chevron Chemical

Eastport, Idaho

- N/A

- N/A

28

0.74

28

|GI RESOURCES INC. Enron Gas Marketing Canada Grand Valley Gas Company

IGI RESOURCES INC.

Enron Gas Marketing Canada Lamb Weston

January
Vol. Price

February

Vol. Price

March

Vol. Price

Quarterly

Total

Vol. Price

Eastport, Idaho

$0 \quad$ N/A

O N/A

1. 0.80

0.80

Eastport, Idaho

$0 \quad$ N/A

0.80

0

0.80

Eastport, Idaho

- N/A

O N/A

20.80

20.80

Sumas, Washington

$\begin{array}{llll}0 & \text { N/A } & 110 & 1.00\end{array}$

O N/A

110

1.00

Eastport, Idaho

- N/A

O N/A

$4 \quad 0.80$

$4 \quad 0.80$

Eastport, Idaho

Eastport, Idaho

- N/A

- N/A

$5 \quad 0.74$

5

0.74

Eastport, Idaho

- N/A

- N/A

$50 \quad 0.74$

50

Eastport, Idaho

- N/A

- N/A

$7 \quad 0.74$

7

0.74 
SHORT-TERM SALES

YEAR: 1995 Quarter: One

Estimated Volumes (MMCF) \& Prices (\$ / MMBTU)
Importer

Seller

Purchaser / End User

IGI RESOURCES INC.

Enron Gas Marketing Cianada PACCO

IGI RESOURCES INC.

Enron Gas Marketing Canada

Pacific Gas \& Electric Company

IGI RESOURCES INC.

Enron Gas Marketing Canada

Sierra Pacific Power Company

IGI RESOURCES INC

Enron Gas Marketing Canada WP Natural Gas

Eastport, Idaho

Enron Gas Marketing Canada Weyerhauser

IGI RESOURCES INC

Equitable

Boise Cascade Corporation

IGI RESOURCES INC.

Equitable

Califomia Industrial End-users

IGI RESOURCES INC.

Equitable

Cascade Natural Gas

IGI RESOURCES INC

Equitable

Chevron Chemical

Eastport, Idaho

$0 \quad$ N/A

$0 \quad$ N/A

$4 \quad 0.65$

$4 \quad 0.65$

IGI RESOURCES INC.

Equitable

Grand Valley Gas Company

Eastport, Idaho

$0 \quad$ N/A

o N/A

$8 \quad 0.65$

$8 \quad 0.65$

IGI RESOURCES INC

Equitable

Lamb Weston
Eastport, Idaho
Eastport, idaho

Eastport, Idaho
anuary
February
Vol. Price

March

Vol. Price

$\begin{aligned} & \text { Quarterly } \\ & \text { Total } \\ & \text { Avg. } \\ & \text { Vol. Price }\end{aligned}$

$0 \quad 0.74$

$0 \quad 0.74$

$20 \quad 0.74$

$0 \quad 1.30$

10.65

Eastport, Idaho

$0 \quad$ N/A

$3 \quad 0.65$

0.65
- N/A

0.65

1

0.65 
SHORT-TERM SALES

YEAR: 1995 Quarter: One

Estimated Volumes (MMCF) \& Prices (\$ / MMBTU)
Importer

Seller

Purchaser/ End User

IGI RESOURCES INC

Equitable

PACCO

IGI RESOURCES INC

Equitable

Pacific Gas \& Electric Company

IGI RESOURCES INC.

Equitable

Sierra Pacific Power Company

IGI RESOURCES INC.

Equitable

WP Natural Gas

Eastport, Idaho

$0 \quad$ N/A

o N/A

$3 \quad 0.65$

$3 \quad 0.65$

IGI RESOURCES'INC

Equitable

Weyerhauser

Eastport, Idaho

- N/A

- N/A

$5 \quad 0.65$

$5 \quad 0.65$

IGI RESOURCES INC.

Mobil Oil Canada Ltd.

Amalgamated Sugar Company

IGI RESOURCES INC.

Mobil Oil Canada Ltd.

American Fine Foods

IGI RESOURCES INC.

Mobil Oil Canada Ltd.

Armstrong World Industries, Inc.

Sumas, Washington

$67 \quad 0.77$

$62 \quad 1.03$

69

1.13

199

0.98

IGI RESOURCES INC

Mobil Oil Canada Ltd.

Ash Grove Cement

Sumas, Washington

16

0.77

$4 \quad 1.03$

$0 \quad 1.13$

$20 \quad 0.82$

IGI RESOURCES INC.

Mobil Oll Canada Ltd.

Ash Grove Cement West

IGI RESOURCES INC.

Mobil Oil Canada Ltd.

Boeing Company
Sumas, Washington

Sumas, Washington $\begin{array}{llll}11 & 0.77 & 22 & 1.03\end{array}$

17

1.13

$50 \quad 1.01$

$8 \quad 0.77$

O N/A

118

1.13 
SHORT-TERM SALES

YEAR: 1995 Quarter: One

Estimated Volumes (MMCF) \& Prices ( $/$ MMBTU)
Importer

Seller

Purchaser/End User

IGI RESOURCES INC.

Mobil Oil Canada Ltd.

Boise Cascade Corporation

IGI RESOURCES INC.

Mobil Oil Canada Ltd.

Boise Cascade Corporation

IGI RESOURCES INC.

Mobil Oil Canada Ltd.

Bullseye Glass

IGI RESOURCES INC.

Mobil Oil Canada Ltd.

Califomia Industrial End-Users

IGI RESOURCES INC

Mobil Oil Canada Ltd.

Califomia Industrial End-users

IGI RESOURCES INC

Mobil Oil Canada Ltd.

Califomia Industrial End-users

IGI RESOURCES INC.

Mobil Oil Canada Ltd.

Camation Dairies

IGI RESOURCES INC.

Mobil Oil Canada Ltd.

Cascade Natural Gas

IGI RESOURCES INC

Mobil Oil Canada Ltd.

Chevron Chemical

IGI RESOURCES INC:

Mobil Oil Canada Ltd.

Darling Delaware

IGI RESOURCES INC:

Mobil Oil Canada Ltd.

Dyno Polymers

\section{Point}

Entry

Eastport, Idaho

Sumas, Washington

Sumas, Washington

Sumas, Washington

Eastport, Idaho

Sumas, Washington

Sumas, Washington

- N/A

o N/A

24

1.13

24

1.13

Eastport, Idaho

Eastport, Idaho

Sumas, Washington

$\begin{array}{ll}10 & 0.77\end{array}$

$9 \quad 1.03$

$0 \quad 1.13$

19

0.89
Sumas, Washington
$3 \quad 0.77$

$3 \quad 1.03$

$0 \quad 1.13$

6

0.90 
SHORT-TERM SALES

YEAR: 1995 Quarter: One

Estimated Volumes (MMCF) \& Prices ( $\$$ / MMBTU)
Importer

Seller

Purchaser / End User

IGI RESOURCES INC. Mobil Oil Canada Ltd.

ESCO Corporation

IGI RESOURCES INC. Mobil Oil Canada Ltd.

Fincrest Schools

IGI RESOURCES INC.

Mobil Oil Canada Ltd.

Grand Valley Gas Company

IGI RESOURCES INC Mobil Oil Canada Ltd.

Interox

IGI RESOURCES INC. Mobil Oil Canada Ltd.

J.R. Simplot Company

IGI RESOURCES INC. Mobil Oil Canada Ltd.

J.R. Simplot Company

IGI RESOURCES INC. Mobil Oil Canada Ltd. James Hardy

Sumas, Washington

GI RESOURCES INC. Mobil Oil Canada Ltd. Jorgensen Steel

IGI RESOURCES INC Mobil Oil Canada Ltd. KTM

IGI RESOURCES INC. Mobil Oil Canada Ltd. Lamb Weston

Eastport, Idaho

IGI RESOURCES INC. Mobil Oil Canada Ltd. Legacy Health System

\section{Point \\ Entry}

Sumas, Washington

Sumas, Washington

Eastport, Idaho

Sumas, Washington

Sumas, Washington

Sumas, Washington

Sumas, Washington

Sumas, Washington
136

0.77

131

1.03

126

1.13

393

0.97
January
Vol. Price

March

Vol. Price

uarterty

Total

Avg.

Vol. Price

168

130

2.42

$166 \quad 2.40$

465

$82 \quad 1.64$

$38 \quad 2.42$

- N/A

120

1.89

255

$156 \quad 2.06$

$90 \quad 2.26$

$501 \quad 1.44$
30 $\quad 0.77$

$21 \quad 1.03$

- N/A

0.88 
SHORT-TERM SALES

YEAR: 1995 Quarter: One

Estimated Volumes (MMCF) \& Prices (\$ / MMBTU)
Importer

Seller

Purchaser / End User

IGI RESOURCES INC. Mobil Oil Canada Lid.

Ore-lda

IGI RESOURCES INC.

Mobil Oil Canada Ltd.

Oroweat

IGI RESOURCES INC.

Mobil Oil Canada Ltd.

PACCO

IGI RESOURCES INC

Mobil Oil Canada Ltd.

Pacific Gas \& Electric Company

IGI RESOURCES INC.

Mobil Oil Canada Ltd.

Portland General Electric Company

IGI RESOURCES INC

Mobil Oil Canada Ltd.

Portland General Electric Company

Sumas, Washington

135

IGI RESOURCES INC

Mobil Oil Canada Ltd.

Roseburg Lumber

Sumas, Washington

18

IGI RESOURCES INC.

Mobil Oil Canada Ltd.

Sierra Pacific Power Company

Eastport, Idaho

- N/A

IGI RESOURCES INC.

Mobil Oil Canada Ltd.

Sierra Pacific Power Company

IGI RESOURCES INC.

Mobil Oil Canada Ltd.

Southwest Gas Corporation

Sumas, Washington

- N/A

IGI RESOURCES INC.

Mobil Oil Canada Ltd.

Universal Frozen Foods
Eastport, Idaho
$10 \quad 2.42$

- N/A

15

2.17
0.77

O N/A

- N/A

135

0.77

0.77

15

1.03

$6 \quad 1.13$

39

0.92

$4 \quad 2.42$

$0 \quad 2.40$

5

2.42

O N/A

$15 \quad 1.13$

15

1.13

Quarterly

Avg.

Vol. Price

$27 \quad 1.04$

o 2.40

$43 \quad 2.14$

$81 \quad 1.00$

$1.04 .2 .10,2 / A$




\section{SHORT-TERM SALES \\ YEAR: 1995 Quarter: One \\ Estimated Volumes (MMCF) \& Prices (\$ / MMBTU)}

Importer

Seller

Purchaser/End User

IGI RESOURCES INC.

Mobil Oil Canada Ltd.

Universal Frozen Foods

IGI RESOURCES INC.

Mobil Oil Canada Ltd.

WP Natural Gas

IGI RESOURCES INC.

Mobil Oil Canada Ltd.

WP Natural Gas

IGI RESOURCES INC.

Mobil Oil Canada Ltd.

Washington Water Power Company

Sumas, Washington

- N/A

- N/A

$117 \quad 1.13$

$117 \quad 1.13$

IGI RESOURCES INC.

Mobil Oil Canada Ltd.

Weyerhauser

Eastport, ldaho

$59 \quad 1.64$

$28 \quad 2.42$

106

2.40

193

2.17

IGI RESOURCES INC.

Mobil Oil Canada Ltd.

Weyerhauser

Sumas, Washington

O N/A

O N/A

$9 \quad 1.13$

$9 \quad 1.13$

IGI RESOURCES INC.

Mobil Oil Canada Ltd.

Willamette

Sumas, Washington

$\begin{array}{ll}72 & 0.77\end{array}$

$41 \quad 1.03$

59

1.13

171

0.96

IGI RESOURCES INC.

Northridge Petroleum Marketing, Inc Boise Cascade Corporation

Eastport, Idaho

- N/A

O N/A

$2 \quad 2.54$

$2 \quad 2.54$

IGI RESOURCES INC.

Northridge Petroleum Marketing, Inc Califomia Industrial End-users

IGI RESOURCES INC.

Northridge Petroleum Marketing, Inc Cascade Natural Gas

Eastport, Idaho

$41 \quad 1.57$

$43 \quad 1.54$

36

1.27

120

1.47

IGI RESOURCES INC.

Northridge Petroleum. Marketing, Inc Chevron Chemical
Eastport, Idaho
$63 \quad 1.54$

1.27 
SHORT-TERM SALES

YEAR: 1995 Quarter: One

Estimated Volumes (MMCF) \& Prices (\$ / MMBTU)
Importer

Seller

Purchaser/End User

IGI RESOURCES INC.

Northridge Petroleum Narketing, Inc Grand Valley Gas Company

IGI RESOURCES INC

Northridge Petroleum Marketing, Inc J.R. Simplot Company

IGI RESOURCES INC.

Northridge Petroleum Marketing, Inc Lamb Weston

IGI RESOURCES INC.

Northridge Petroleum Marketing, Inc PACCO

IGI RESOURCES INC

Northridge Petroleum Marketing, Inc Pacific Gas \& Electric Company

IGI RESOURCES INC

Northridge Petroleum Marketing, Inc Portland General Electric Company

IGI RESOURCES INC

Northridge Petroleum Marketing, Inc Sierra Pacific Power Company

IGI RESOURCES INC

Northridge Petroleum Marketing, Inc

Universal Frozen Foods

IGI RESOURCES INC.

Northridge Petroleum Marketing, Inc WP Natural Gas

IGI RESOURCES INC

Northridge Petroleum Marketing, Inc Weyerhauser

IGI RESOURCES INC

Petro-Canada Hydrocarbons Inc.

Boise Cascade Corporation
Eastport, Idaho

$5 \quad 1.57$

7. 1.54

O N/A

$12 \quad 1.55$

Eastport, Idaho

$$
\begin{aligned}
& \text { Point } \\
& \text { of } \\
& \text { Entry }
\end{aligned}
$$

Eastport, Idaho

Eastport, Idaho

$92 \quad 1.57$

$14 \quad 1.57$

26

1.54

$14 \quad 1.27$

55

1.48

Eastport, Idaho

$51 \quad 1.57$

$97 \quad 1.57$

108

1.54

- N/A

205

1.55

Eastport, Idaho

- N/A

$3 \quad 1.54$

o $\quad 1.27$

$3 \quad 1.52$
Eastport, ldaho
Quarterly
Total
Avg.
Vol. Price

$378 \quad 1.48$

$0 \quad 1.27$

$68 \quad 1.50$

Eastport, Idaho

$\begin{array}{ll}66 & 1.57\end{array}$

$19 \quad 1.54$

63

1.27

149

1.44

- N/A

$0 \quad 1.60$

1.60 
Importer

Purchaser / End User

IGI RESOURCES INC.

Petro-Canada Hydrocarbons Inc. Califomia Industrial End-users

IGI RESOURCES INC.

Petro-Canada Hydrocarbons Inc. Cascade Natural Gas

IGI RESOURCES INC.

Petro-Canada Hydrocarbons Inc. Chevron Chemical

IGI RESOURCES INC.

Petro-Canada Hydrocarbons Inc. Grand Valley Gas Company

IGI RESOURCES INC.

Petro-Canada Hydrocarbons Inc. J.R. Simplot Company

IGI RESOURCES INC.

Petro-Canada Hydrocarbons Inc. Lamb Weston

IGI RESOURCES INC.

Petro-Canada Hydrocarbons Inc.

PACCO

IGI RESOURCES INC.

Petro-Canada Hydrocarbons Inc. Pacific Gas \& Electric Company

IGI RESOURCES INC.

Petro-Canada Hydrocarbons Inc.

Portland General Electric Company

IGI RESOURCES INC.

Petro-Canada Hydrocarbons Inc.

Sierra Pacific Power Company

IGI RESOURCES INC.

Petro-Canada Hydrocaibons Inc.

Universal Frozen Foods
Eastport, ldaho

\section{Point \\ Entry}

Eastport, Idaho

Eastport, Idaho

Eastport, Idaho

Eastport, Idaho

Eastport, Idaho

Eastport, Idaho

Eastport, Idaho

Eastport, Idaho

Eastport, Idaho

Eastport, Idaho
SHORT-TERM SALES

EAR: 1995 Quarter: One

Volumes (MMCF) \& Prices (\$ / MMBTU) 
SHORT-TERM SALES

YEAR: 1995 Quarter: One

Estimated Volumes (MMCF) \& Prices (\$/MMBTU)
Importer

Seller

Purchaser / End User

GI RESOURCES INC

Petro-Canada Hydrocarbons Inc.

WP Natural Gas

IGI RESOURCES INC.

Petro-Canada Hydrocarbons Inc.

Weyerhauser

IGI RESOURCES INC.

Petro-Canada Resources

Califomia Industrial End-Users

Eastport, Idaho

IGI RESOURCES INC.

Petro-Canada Resources

Chevron Chemical

IGI RESOURCES INC.

Petro-Canada Resources

J.R. Simplot Company

IGI RESOURCES INC

Petro-Canada Resources

Lamb Weston

IGI RESOURCES INC

Petro-Canada Resources

Portland General Electric Company

Eastport, Idaho

IGI RESOURCES INC

Petro-Canada Resources

Universal Frozen Foods

IGI RESOURCES INC.

Petro-Canada Resources

Weyerhauser

Eastport, Idaho

$1 \quad 1.00$

o N/A

$0 \quad$ N/A

1

1.00

IGI RESOURCES INC.

Summit Resources Linnited

Califomia Industrial Erid-users

IGI RESOURCES INC.

Summit Resources Lirnited

Cascade Natural Gas

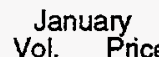

23

0.96

$5 \quad 0.66$

$0 \quad$ N/A

$0 \quad N / A$

$3 \quad 1.00$

Eastport, Idaho

Eastport, Idaho

Eastport, Idaho

$38 \quad 0.94$

$0 \quad N / A$

N/A

38

0.94

Eastport, Idaho
$10 \quad 0.94$

O N/A

$0 \quad N / A$

10

0.94 
SHORT-TERM SALES

YEAR: 1995 Quarter: One

Estimated Volumes (MMCF) \& Prices (\$ / MMBTU)
Importer

Seller

Purchaser / End User

IGI RESOURCES INC

Summit Resources Limited Chevron Chemical

IGI RESOURCES INC.

Summit Resources Limited

Grand Valley Gas Company

[G] RESOURCES INC

Summit Resources Limited

J.R. Simplot Company

IGI RESOURCES INC.

Summit Resources Limited

Lamb Weston

Eastport, Idaho

$4 \quad 0.94$

- N/A

$0 \quad$ N/A

4

0.94

IGI RESOURCES INC.

Summit Resources Limited

Pacific Gas \& Electric Company

IGI RESOURCES INC.

Summit Resources Limited

Portland General Electric Company

Eastport, Idaho

24

0.94

- N/A

O N/A

$24 \quad 0.94$

IGI RESOURCES INC.

Summit Resources Limited

Universal Frozen Foods

IGI RESOURCES INC

Summit Resources Limited

WP Natural Gas

Eastport, Idaho

$4 \quad 0.94$

O N/A

- N/A

$4 \quad 0.94$

IGI RESOURCES INC

Summit Resources Limited Weyertauser

Eastport, Idaho

0.94

- N/A

$0 \quad$ NIA

17

0.94

IGI RESOURCES INC

Talisman Energy, Inc.

Basic American Foods

IGI RESOURCES INC

Talisman Energy, Inc.

Boise Cascade Corporation
Sumas, Washington

O N/A

- N/A

$1 \quad 1.07$
$0 \quad$ N/A

$0 \quad$ N/A

21.07

$2 \quad 1.07$ 
SHORT-TERM SALES

YEAR: 1995 Quarter: One

Estimated Volumes (MMCF) \& Prices (\$ / MMBTU)
Importer

Seller

Purchaser / End User

IGI RESOURCES INC. Talisman Energy, Inc.

Bulliseye Glass

IGI RESOURCES INC. Talisman Energy, Inc. IBP

IGI RESOURCES INC. Talisman Energy, Inc. J.R. Simplot Company

IGI RESOURCES INC.

Talisman Energy, Inc.

Roseburg Lumber

IGI RESOURCES INC.

Talisman Energy, Inc.

Sierra Pacific Power Company

IGI RESOURCES INC.

Talisman Energy, Inc.

Willamette

IGI RESOURCES INC.

Wascana Energy Marketing Inc.

Boise Cascade Corporation

IGI RESOURCES INC.

Wascana Energy Marketing Inc. Califomia Industrial End-users

IGI RESOURCES INC.

Wascana Energy Marketing Inc.

Cascade Natural Gas

IGI RESOURCES INC.

Wascana Energy Marketing Inc. Chevron Chemical

IGI RESOURCES INC.

Wascana Energy Marketing Inc.

Grand Valley Gas Company
Sumas, Washington

$$
\begin{gathered}
\text { Point } \\
\text { of } \\
\text { Entry }
\end{gathered}
$$

Sumas, Washington

Sumas, Washington

Sumas, Washington

Sumas, Washington

Eastport, Idaho

Eastport, Idaho

Eastport, Idaho

Eastport, Idaho

Eastport, Idaho
- N/A

- N/A

$11 \quad 1.07$

$11 \quad 1.07$

$\begin{aligned} & \text { January } \\ & \text { Vobruary }\end{aligned}$
Vol. Price Price

March

Vol. Price

Quarterly
Total
Avg.
Vol. Price

- N/A

- N/A

$0 \quad 1.07$

$0 \quad 1.07$

$245 \quad 1.47$

174

1.13

230

2.14

$650 \quad 1.62$

- N/A

- N/A

$1 \quad 1.07$

$\begin{array}{ll}1 & 1.07\end{array}$

- N/A

$40 \quad 1.13$

- N/A

40

- N/A

- N/A

$0 \quad 1.56$

$0 \quad 1.56$

$57 \quad 1.81$

o N/A

$2 \quad 0.78$

60

1.77

$15 \quad 1.81$

21.55

$8 \quad 0.78$

$26 \quad 1.45$

$25 \quad 1.81$

$3 \quad 1.55$

13

0.78

$41 \quad 1.47$

$71 \quad 1.81$

$4 \quad 1.55$

23

0.78

98

1.56 
Importer

Seller

Purchaser / End User

IGI RESOURCES INC.

Wascana Energy Marketing Inc. J.R. Simplot Company

IGI RESOURCES INC.

Wascana Energy Marketing Inc. Lamb Weston

Eastport, Idaho

$1 \quad 1.55$

$3 \quad 0.78$

10

1.43

IGI RESOURCES INC.

Wascana Energy Marketing Inc.

PACCO

Eastport, Idaho

$0 \quad$ N/A

O N/A

$0 \quad 0.78$

0

IGI RESOURCES INC.

Wascana Energy Marketing inc.

Pacific Gas \& Electric Company

IGI RESOURCES INC.

Wascana Energy Marketing inc.

Portland General Electric Company

Eastport, Idaho

36

1.81

$5 \quad 1,55$

O N/A

$42 \quad 1.78$

IGI RESOURCES INC.

Wascana Energy Marketing Inc.

Sierra Pacific Power Company

Eastport, Idaho

o N/A

$0 \quad 1.55$

0.78

$0 \quad 1.31$

IGI RESOURCES INC.

Wascana Energy Marketing Inc.

Universal Frozen Foods

Eastport, Idaho

$2 \quad 1.81$

$0 \quad 1.55$

$0 \quad N / A$

$2 \quad 1.77$

IGI RESOURCES INC.

Wascana Energy Marketing Inc. WP Natural Gas

Eastport, Idaho

$6 \quad 1.81$

$4 \quad 1.55$

$9 \quad 0.78$

$19 \quad 1.25$

IGI RESOURCES INC.

Wascana Energy Marketing Inc.

Weyerhauser

Eastport, Idaho

1.81

$1 \quad 1.55$

$15 \quad 0.78$

$41 \quad 1.43$

IGI RESOURCES INC.

Westcoast Energy Marketing, Ltd.

Boise Cascade Corporation

Eastport, Idaho

- N/A

$0 \quad$ N/A

$0 \quad 3.52$

$0 \quad 3.52$

IGI RESOURCES INC

Westcoast Energy Marketing, Ltd.

Califomia Industral End-users
Eastport, Idaho o N/A

11.76

15

1.59 
SHORT-TERM SALES

YEAR: 1995 Quarter: One

Estimated Volumes (MMCF) \& Prices (\$ / MMBTU)
Importer

Seller

Purchaser/End User

IGI RESOURCES INC.

Westcoast Energy Marketing, Ltd.

Cascade Natural Gas

IGI RESOURCES INC.

Westcoast Energy Marketing, Ltd. Chevron Chemical

IGI RESOURCES INC.

Westcoast Energy Marketing, Ltd.

Grand Valley Gas Company

IGI RESOURCES INC.

Westcoast Energy Marketing, Ltd. J.R. Simplot Company

IGI RESOURCES INC.

Westcoast Energy Marketing, Ltd. Lamb Weston

IGI RESOURCES INC.

Westcoast Energy Marketing, Ltd. PACCO

IGI RESOURCES INC

Westcoast Energy Marketing, Ltd.

Pacific Gas \& Electric Company

IGI RESOURCES INC

Westcoast Energy Marketing, Ltd.

Portland General Electric Company

[G] RESOURCES INC

Westcoast Energy Marketing, Ltd.

Sierra Pacific Power Company

IGI RESOURCES INC

Westcoast Energy Marketing, Ltd.

Universal Frozen Foods

Eastport, Idaho

$0 \quad 1.58$

$1 \quad 1.57$

$0 \quad$ N/A

1

1.57

IGI RESOURCES INC.

Westcoast Energy Marketing, Ltd. WP Natural Gas
Eastport, Idaho
Eastport, Idaho

Eastport, Idaho

$0 \quad$ N/A

- N/A

$0 \quad 1.76$

$0 \quad 1.76$

Quarterly

Avg.

Vol. Price

Vol. Price

$12 \quad 1.64$

$36 \quad 1.63$

$10 \quad 1.58$

$5 \quad 1.63$

$6 \quad 1.63$

$18 \quad 1.57$

Eastport, ldaho

- N/A

$0 \quad 1.57$

$0 \quad 1.76$

$0 \quad 1.59$ $\begin{array}{ll}7 & 1.57\end{array}$

$5 \quad 1.76$

$13 \quad 1.64$ 
SHORT-TERM SALES

YEAR: 1995 Quarter: One

Estimated Volumes (MMCF) \& Prices (\$ / MMBTU)
Importer

Seller

Purchaser / End User

IGI RESOURCES INC.

Westcoast Energy Marketing, Ltd.

Weyerhauser

IGI RESOURCES INC.

Western Gas Marketing Limited

Boise Cascade Corporation

IGI RESOURCES INC.

Western Gas Marketing Limited Califomia Industrial End-users

IGI RESOURCES INC.

Western Gas Marketing Limited Cascade Natural Gas

IGI RESOURCES INC.

Western Gas Marketing Limited Chevron Chemical

IGI RESOURCES INC.

Western Gas Marketing Limited Grand Valley Gas Company

IGI RESOURCES INC.

Western Gas Marketing Limited J.R. Simplot Company

IGI RESOURCES INC.

Western Gas Marketing Limited Lamb Weston

Eastport, Idaho

$5 \quad 0.84$

$9 \quad 0.85$

$8 \quad 0.81$

23

0.83

IGI RESOURCES INC.

Western Gas Marketing Limited PACCO

Eastport, Idaho

O N/A

- N/A

$\begin{array}{ll}0 & 0.81\end{array}$

$0 \quad 0.81$

IGI RESOURCES INC.

Westem Gas Marketing Limited

Pacific Gas \& Electric Company

IGI RESOURCES INC.

Western Gas Marketing Limited

Portland General Electric Company

January
Vol. Price

February

Vol. Price

March

Vol. Price

Quarterly

Total

Vol. ${ }^{\text {Avg. }}$ Price

Eastport, Idaho

$2 \quad 1.57$

$7 \quad 1.76$

15

1.67

Eastport, Idaho

- N/A

$1 \quad 1.62$

$1 \quad 1.62$

Eastport, Idaho

$55 \quad 0.84$

$6 \quad 0.81$

61

0.84

Eastport, Idaho

$15 \quad 0.84$

$16 \quad 0.85$

$20 \quad 0.81$

51

0.83

Eastport, Idaho

$24 \quad 0.84$

$22 \quad 0.85$

$31 \quad 0.81$

78

0.83

Eastport, Idaho

$33 \quad 0.84$

$0 \quad N / A$

42

0.84

Eastport, Idaho

$18 \quad 0.84$

- N/A

$10 \quad 0.81$

28

0.83

Eastport, Idaho

$35 \quad 0.84$

$39 \quad 0.85$

- N/A

74

0.85 
SHORT-TERM SALES

YEAR: 1995 Quarter: One

Estimated Volumes (MMCF) \& Prices (\$ / MMBTU)
Importer

Seller

Purchaser / End User

IGI RESOURCES INC.

Western Gas Marketing Limited

Sierra Pacific Power Company

IGI RESOURCES INC.

Western Gas Marketing Limited

Universal Frozen Foods

IGI RESOURCES INC

Western Gas Marketing Limited WP Natural Gas

IGI RESOURCES INC

Western Gas Marketing Limited

Weyerhauser

\section{Point}

Entry

Eastport, Idaho

Eastport, Idaho

Eastport, !daho

Eastport, Idaho

Niagara Falls, NY

Continental Energy Marketing Ltd.

Indeck Energy Services of Oswego

INDECK ENERGY SERVICES, INC.

Continental Energy Marketing Ltd.

indeck Energy Services of Yerkes

\author{
January
}

Vol. Price

February

Vol. Price

March

Vol. Price

Quarterly
Total

Total

Vol.

Price

INLAND PACIFIC ENERGY SERVICES CORP.

Wainoco Oil Corporation

Potlatch Corporation

Sumas, Washington

INLAND PACIFIC ENERGY SERVICES LTD.

Inland Pacific Energy Services Ltd.

American Hunter Energy

Sumas, Washington

INLAND PACIFIC ENERGY SERVICES LTD.

Inland Pacific Energy Services Ltd.

Cascade Natural Gas

Sumas, Washington

$0 \quad$ N/A

$0 \quad N / A$

154

0.99

154

0.99

INLAND PACIFIC ENERGY SERVICES LTD.

Inland Pacific Energy Services Ltd.

Coastal Gas Marketing Company

Sumas, Washington

- N/A

$254 \quad 1.05$

$273 \quad 1.07$

528

1.06

INLAND PACIFIC ENERGY SERVICES LTD.

Inland Pacific Energy. Services Ltd.

Enron Gas Marketing

Sumas, Washington
$10 \quad 0.92$

$94 \quad 1.10$

$34 \quad 1.07$

138

1.08 
SHORT-TERM SALES

YEAR: 1995 Quarter: One

Estimated Volumes (MMCF) \& Prices (\$ / MMBTU)
Importer

Seller

Purchaser/End User

INLAND PACIFIC ENERGY SERVICES LTD.

Inland Pacific Energy Services Ltd.

IGI Resources

Sumas, Washington

INLAND PACIFIC ENERGY SERVICES LTD.

Inland Pacific Energy Services Ltd.

Inland Pacific

Sumas, Washington

INLAND PACIFIC ENERGY SERVICES LTD.

Inland Pacific Energy Services Ltd.

Interenergy Corporation

Sumas, Washington

INLAND PACIFIC ENERGY SERVICES LTD.

Inland Pacific Energy Services Ltd.

Kimball Energy Corporation

Sumas, Washington

INLAND PACIFIC ENERGY SERVICES LTD.

Inland Pacific Energy Services Ltd.

Koch Gas Services Company

Sumas, Washington

INLAND PACIFIC ENERGY SERVICES LTD.

Inland Pacific Energy Services Ltd.

Mock Resources

Sumas, Washington

INLAND PACIFIC ENERGY SERVICES LTD.

Inland Pacific Energy Services Ltd.

National Gas \& Electric

Sumas, Washington

INLAND PACIFIC ENERGY SERVICES LTD.

Inland Pacific Energy Services Ltd.

Northwest Natural Gas Company

Sumas, Washington

INLAND PACIFIC ENERGY SERVICES LTD.

Inland Pacific Energy Services Ltd.

Pacific Gas \& Electric Company

Sumas, Washington

INLAND PACIFIC ENERGY SERVICES LTD.

Inland Pacific Energy Services Ltd.

Sierra Pacific Power Company

Sumas, Washington

INLAND PACIFIC ENERGY SERVICES LTD.

Inland Pacific Energy Services Ltd.

Southwest Gas Comoration

Sumas, Washington

January
Vol. Price
Vol. Price

February

March

Quarterly

Total

Vol. Pvg. Price

Vol. Price Vol Price

- N/A

10

1.12

17

0.99

27

1.04

$205 \quad 1.46$

$56 \quad 1.03$

$126 \quad 1.01$

387

1.25

O N/A

$5 \quad 1.00$

- N/A

5

1.00

$4 \quad 1.00$

$0 \quad N / A$

- N/A

4

1.00

- N/A

120

1.01

146

1.01

26

1.01

- N/A

$101 \quad 1.03$

$0 \quad$ N/A

$101 \quad 1.03$

- N/A

155

1.07

155

1.07

$80 \quad 0.98$

$14 \quad 1.02$

21

115

0.99

- N/A

- N/A

574

1.02

574

1.02

$188 \quad 1.50$

48

1.05

488

1.05

1160

1.12

$39 \quad 0.91$

$6 \quad 1.12$

$64 \quad 1.08$

108

1.02 
SHORT-TERM SALES

YEAR: 1995 Quarter: One

Estimated Volumes (MMCF) \& Prices (\$/MMBTU)
Importer

Seller

Purchaser / End User

INLAND PACIFIC ENERGY SERVICES LTD.

inland Pacific Energy Services Ltd.

Teco Gas Marketing

Sumas, Washington

Sumas, Washington

Inland Pacific Energy Services Ltd.

Washington Water Power Company

INTALCO ALUMINUM CORPORATION

CanWest Gas Supply Inc.

Intalco Aluminum Corporation

INTERENERGY CORFORATION

Ocelot Energy, Inc.

Interenergy Corporation

Point

Entry

January

Vol. Price

February

Vol. Price

March

Vol. Price

Quarterly

Total

Avg

Vol. Price

$40 \quad 1.49$

39

1.14

$42 \quad 1.22$

122

1.28

INVERNESS RESOURCES INC.

Invemess Petroleum Ltd.

Mock Resources

Eastport, Idaho

$117 \quad 1.90$

105

1.23

$117 \quad 1.22$

339

1.46

IROQUOIS ENERGY MANAGEMENT, INC.

Renaissance Energy Lid.

SUNY - N.Y.

Niagara Falls, NY

323

1.80

292

1.80

323

1.80

937

1.80

JMC FUEL SERVICES, INC.

Northridge Gas Marketing

JMC Fuel Services

Waddington, NY

$0 \quad$ N/A

$10 \quad 1.60$

$0 \quad N / A$

10

1.60

JONAN GAS MARKETING Jonan Gas Marketing L.td.

Chooljian Brothers

Eastport, Idaho

31.00

$0 \quad$ N/A

$0 \quad$ N/A

1.00

JONAN GAS MARKETING

Jonan Gas Marketing Ltd.

Del Ray Packing

Eastport, Idaho

$6 \quad 1.00$

$0 \quad$ N/A

- N/A

$6 \quad 1.00$

JONAN GAS MARKETING

Jonan Gas Marketing Ltd.

Enron Gas Marketing

Eastport, Idaho

$21 \quad 1.00$

- N/A

- N/A

21

1.00

JONAN GAS MARKETING Jonan Gas Marketing Ltd.

Mock Resources

Eastport, Idaho
$90 \quad 1.00$

- N/A

0

N/A

90

1.00 
SHORT-TERM SALES

YEAR: 1995 Quarter: One

Estimated Volumes (MMCF) \& Prices (\$ / MMBTU)
Importer

Seller

Purchaser / End User

JONAN GAS MARKETING

Jonan Gas Marketing Ltd.

Rogers Foods

JONAN GAS MARKETING

Jonan Gas Marketing Ltd.

Salwasser

KCS ENERGY MARKETING, INC.

Ramarto Resources

KCS Energy Management

Grand Island, NY

$2 \quad 2.16$

O N/A

$0 \quad$ N/A

$2 \quad 2.16$

KCS ENERGY MARKETING, INC.

Renaissance Energy Ltd.

KCS Energy Management

KCS ENERGY MARKETING, INC.

Renaissance Energy Ltd.

KCS Energy Management

Niagara Falls, NY

$0 \quad$ N/A

11

1.79

O N/A

$11 \quad 1.79$

KCS ENERGY MARKETING, INC.

Renaissance Energy Ltd.

KCS Energy Management

Noyes, Minnesota

$160 \quad 1.09$

140

1.10

$156 \quad 1.02$

456

1.07

KIMBALL ENERGY CORPORATION Canadian Hydrocarbons Marketing

Canwest Gas Supply

Sumas, Washington

$0 \quad$ N/A

15

1.04

O N/A

15

1.04

KIMBALL ENERGY CORPORATION Canadian Hydrocarbons Marketing Greeley Gas Company

Sumas, Washington

$3 \quad 0.99$

$8 \quad 1.00$

o N/A

$10 \quad 1.00$

KIMBALL ENERGY CORPORATION Canadian Hydrocarbons Marketing IGI Resources

Sumas, Washington

196

1.11

216

1.11

629

1.17

KIMBALL ENERGY CORPORATION Canadian Hydrocarbons Marketing Inland Pacific

Sumas, Washington

- N/A

20

1.03

$0 \quad N / A$

20

1.03

KIMBALL ENERGY CORPORATION Canadian Hydrocarbons Marketing Kimball Energy Corporation
Sumas, Washington

$11 \quad 1.43$

- N/A

- N/A

11

1.43 


\section{SHORT-TERM SALES}

YEAR: 1995 Quarter: One

Estimated Volumes (MMCF) \& Prices ( $\$$ / MMBTU)
Importer

Seller

Purchaser / End User

KIMBALL ENERGY CORPORATION Canadian Hydrocarbons Marketing Mock Resources

KIMBALL ENERGY CORPORATION Canadian Hydrocarbons Marketing Natural Gas Clearinghouse

KIMBALL ENERGY CORPORATION Canadian Hydrocarbons Marketing North Canadian Marketing Corp.

KIMBALL ENERGY CORPORATION Canadian Hydrocarbons Marketing Southwest Gas Corporation

Sumas, Washington

25

January
Vol. Price

February

of

Sumas, Washington

Sumas, Washington

$28 \quad 1.01$

- N/A

- N/A

$28 \quad 1.01$

Sumas, Washington

$6 \quad 1.00$

- N/A

$0 \quad$ N/A

$6 \quad 1.00$

KIMBALL ENERGY CORPORATION Canadian Hydrocarbons Marketing Texas-Ohio Gas, Inc.

Sumas, Washington

$30 \quad 1.20$

$0 \quad$ N/A

- N/A

30

1.20

KOCH GAS SERVICES COMPANY

Koch Gas Services Canada

Koch Gas Services Company

Noyes, Minnesota

- N/A

- N/A

$63 \quad 1.09$

63

1.09

KOCH GAS SERVICES COMPANY

Koch Gas Services Canada

Koch Gas Services Company

Port of Morgan, MT

1074

2.16

1037

1.80

1055

1.74

3166

1.90

LOUIS DREYFUS ENE:RGY CORPORATION

Canadian Hunter Marketing Ltd.

Enron Gas Marketing

Niagara Falls, NY

- N/A

$277 \quad 1.60$

$0 \quad$ N/A

277

1.60

LOUIS DREYFUS ENE:RGY CORPORATION

Canadian Hunter Marketing Ltd.

New England Power Company

Waddington, NY

$3 \quad 1.90$

O N/A

$0 \quad$ N/A

$3 \quad 1.90$

LOUIS DREYFUS ENEERGY CORPORATION

Union Gas Limited

Direct Gas Marketing

Grand Island, NY

- N/A

55

2.42

29

2.42

84

2.42
MASSPOWER

Coastal Gas Marketing, Canada

Masspower
Waddington, NY
21.89

O N/A

- N/A

$2 \quad 1.89$ 
SHORT-TERM SALES

YEAR: 1995 Quarter: One

Estimated Volumes (MMCF) \& Prices (\$ / MMBTU)
Importer

Seller

Purchaser / End User

MASSPOWER

ProGas Limited

Masspower

MASSPOWER

Renaissance Energy Ltd.

Masspower

MIDCON GAS SERVICES CORPORATION

Natural Gas Pipeline

CMS Gas Marketing

Noyes, Minnesota

Noyes, Minnesota

Natural Gas Pipeline

Midcon Gas Services Corporation

MIDCON GAS SERVICES CORPORATION

Natural Gas Pipeline

Minnegasco

Noyes, Minnesota

930

1.12

690

1.12

720

1.12

2340

1.12

MIDCON GAS SERVICES CORPORATION

Natural Gas Pipeline

Wisconsin Power \& Light

Noyes, Minnesota

MIDCON GAS SERVICES CORPORATION

Natural Gas Pipeline

Wisconsin Public Service Corp.

Noyes, Minnesota

$0 \quad N / A$

$0 \quad$ N/A

16

1.12

1.12

MIDCON GAS SERVICES CORPORATION

Western Gas Marketing Limited

Madison Gas \& Electric

Noyes, Minnesota

$61 \quad 1.12$

O N/A

$0 \quad N / A$

$61 \quad 1.12$

MOBIL NATURAL GAS INC.

Mobil Natural Gas Canada

Arco Products Company

Sumas, Washington

- N/A

o N/A

$15 \quad 1.39$

$15 \quad 1.39$

MOBIL NATURAL GAS INC.

Mobil Natural Gas Canada

Associated Gas Services, Inc.

Eastport, Idaho

$0 \quad$ N/A

$15 \quad 1.40$

$10 \quad 1.47$

$25 \quad 1.43$

MOBIL NATURAL GAS INC

Mobil Natural Gas Canada

CanStates Gas Marketing
$0 \quad$ N/A

$8 \quad 1.40$

$0 \quad$ N/A

$8 \quad 1.40$ 


\section{SHORT-TERM SALES \\ YEAR: 1995 Quarter: One \\ Estimated Volumes (MMCF) \& Prices ( $\$$ /MMBTU)}

Importer

Seller

Purchaser / End User

MOBIL NATURAL GAS INC.

Mobil Natural Gas Canada

CanStates Gas Marketing

MOBIL NATURAL GAS INC.

Mobil Natural Gas Canada

Cascade Natural Gas

MOBIL NATURAL GAS INC

Mobil Natural Gas Canada

Coastal Gas Marketing Company

MOBIL NATURAL GAS INC.

Mobil Natural Gas Canada

Coastal Gas Marketingl Company

MOBIL NATURAL GAS INC.

Mobil Natural Gas Canada

Cook inlet

Eastport, Idaho

Sumas, Washington

$55 \quad 1.62$

$0 \quad$ N/A

O N/A

55

1.62

MOBIL NATURAL GAS INC.

Mobil Natural Gas Cariada

Enron Gas Marketing

MOBIL NATURAL GAS INC.

Mobil Natural Gas Cariada

IGI Resources

Eastport, Idaho

- N/A

$421 \quad 1.40$

1.47

581

1.42

MOBIL NATURAL GAS INC.

Mobil Natural Gas Carlada

IGI Resources

Sumas, Washington

$1411 \quad 1.62$

$1468 \quad 1.60$

1206

1.39

$4085 \quad 1.54$

MOBIL NATURAL GAS INC.

Mobil Natural Gas Canada

Interenergy Corporation

Sumas, Washington

a N/A

- N/A

$51 \quad 1.39$

51

1.39

MOBIL NATURAL GAS INC.

Mobil Natural Gas Canada

lowa Illinois Gas \& Electric
Port of Morgan, MT
$277 \quad 1.15$

307

1.30

891

1.15 
SHORT-TERM SALES

YEAR: 1995 Quarter: One

Estimated Volumes (MMCF) \& Prices (\$ / MMBTU)
Importer

Seller

Purchaser/End User

MOBIL NATURAL GAS INC

Mobil Natural Gas Canada.

J. Aaron Company

MOBIL NATURAL GAS INC

Mobil Natural Gas Canada

Kimball Energy Corporation

MOBIL NATURAL GAS INC.

Mobil Natural Gas Canada

Koch Gas Services Company

MOBIL NATURAL GAS INC.

Mobil Natural Gas Canada

Minnegasco

Port of Morgan, MT

MOBIL NATURAL GAS INC.

Mobil Natural Gas Canada

Natural Gas Clearinghouse

MOBIL NATURAL GAS INC.

Mobil Natural Gas Canada

Northem Illinois Gas

MOBIL NATURAL GAS INC

Mobil Natural Gas Canada

Northem Minnesota Utilities

MOBIL NATURAL GAS INC.

Mobil Natural Gas Canada

Northwest Natural Gas Company

Sumas, Washington

$213 \quad 1.62$

- N/A

40

1.39

614

1.47

MOBIL NATURAL GAS INC.

Mobil Natural Gas Canada

Novagas Clearinghouse

MOBIL NATURAL GAS INC.

Mobil Natural Gas Canada

Pacific Gas \& Electric Company

MOBIL NATURAL GAS INC.

Mobil Natural Gas Canada

Peoples Natural Gas Company

January

Vol. Price

February

Vol. Price

March

Vol. Price

Quarterty

Total

Avg.

Vol

294

1.61

Sumas, Washington

$23 \quad 1.62$

O N/A

15

1.39

177

1.42

Sumas, Washington

$349 \quad 1.62$

123

1.60

39

626

1.56

Sumas, Washington

o N/A

$24 \quad 1.60$

$\begin{array}{ll}72 & 1.39\end{array}$

$96 \quad 1.44$

Port of Morgan, MT

$552 \quad 1.00$

$498 \quad 1.15$

395

1.30

1445

1.13

Sumas, Washington

o N/A

- N/A

$7 \quad 1.39$

$7 \quad 1.39$

Eastport, Idaho

1.10

$33 \quad 1.40$

$\begin{array}{ll}77 & 1.47\end{array}$

261

1.25

Port of Morgan, MT
$58 \quad 1.15$

$61 \quad 1.30$

183

1.15 


\section{SHORT-TERM SALES \\ YEAR: 1995 Quarter: One}

Estimated Volumes (MMCF) \& Prices (\$ / MMBTU)
Importer

Seller

Purchaser / End User

MOBIL NATURAL GAS INC.

Mobil Natural Gas Canada

Southwest Gas Corporation

MOBIL NATURAL GAS INC. Mobil Natural Gas Canada

Teco Gas Marketing

MOBIL NATURAL GAS INC. Mobil Natural Gas Canada

Tenaska Marketing Ventures

MOBIL NATURAL GAS INC. Mobil Natural Gas Canada

Tosco Northwest

MOBIL NATURAL GASINC.

Mobil Natural Gas Canada

Universal Resources

MOBIL NATURAL GAS INC.

Mobil Natural Gas Canada

Washington Natural Gas Company

Sumas, Washington

- N/A

$0 \quad$ N/A

52

1.39

52

1.39

MOBIL NATURAL GAS INC.

Mobil Natural Gas Canada

Washington Water Power Company

Eastport, Idaho

$312 \quad 1.10$

o N/A

$0 \quad$ N/A

312

1.10

MOBIL NATURAL GASINC.

Mobil Natural Gas Canada

Washington Water Power Company

Sumas, Washington

$27 \quad 1.62$

$6 \quad 1.60$

$4 \quad 1.39$

37

1.59

MURPHY GAS GATHERING

Murphy Oil Company L.td.

Hadson Gas Marketing

NATIONAL FUEL GAS DISTRIBUTION CoEnergy Trading Company

National Fuel Gas Distribution

NATIONAL FUEL GAS DISTRIBUTION Norstar Energy L.P.

National Fuel Gas Distribution
- N/A

- N/A

70

70

1.64 
SHORT-TERM SALES

YEAR: 1995 Quarter: One

Estimated Volumes (MMCF) \& Prices (\$ / MMBTU)
Importer

Seller

Purchaser / End User

NATIONAL FUEL GAS DISTRIBUTION

ProGas Limited

National Fuel Gas Distribution

Grand Island, NY

NATIONAL FUEL GAS DISTRIBUTION

RG\&E

National Fuel Gas Distribution

NATIONAL FUEL GAS DISTRIBUTION

National Fuel Gas Distribution

NATIONAL FUEL GAS DISTRIBUTION

Union Gas Limited

National Fuel Gas Distribution

Grand Island, NY

Entry

Grand Island, NY

Grand Island, NY
Renaissance Energy Ltd.

January
Vol

Detroit, Michigan

Detroit, Michigan

Detroit, Michigan

Direct Energy Marketing Limited

National Steel Corporation

NATIONAL STEEL CORPORATION

Howard Energy Company, Inc.

National Steel Corporation

Detroit, Michigan

Eastport, Idaho

MARKETING IN

Associated Gas Services, Inc.

NORCEN MARKETING INC.

Norcen Energy Resources

Hercules

100

436
Eastport, Idaho
- N/A

$0 \quad N / A$

$6 \quad 1.58$

$6 \quad 1.58$

O N/A

$10 \quad 1.55$

$0 \quad N / A$

$10 \quad 1.55$

$20 \quad 1.55$

- N/A

- N/A

$20 \quad 1.55$

$0 \quad$ N/A

$25 \quad 1.55$

$10 \quad 1.58$

$35 \quad 1.56$

2.34

$138 \quad 2.55$

$153 \quad 2.43$

595

2.41

$0 \quad$ N/A

$0 \quad$ N/A

100

2.41

2.29

560

2.13

491

2.13

1487

2.18

- N/A

- N/A

$7 \quad 1.51$

$7 \quad 1.51$

$902 \quad 133$

$1094 \quad 0.86$

1417

0.90

$3412 \quad 1.00$ $\begin{array}{llll}0 & \text { N/A } & 187 & 0.79\end{array}$

- N/A

187

0.79 


\section{SHORT-TERM SALES}

YEAR: 1995 Quarter: One

Estimated Volumes (MMCF) \& Prices (\$ / MMBTU)
Importer

Seller

Purchaser / End User

NORCEN MARKETING INC.

Norcen Energy Resources

Pacific Gas \& Electric Company

NORSTAR ENERGY, L.P.

CoEnergy Trading Company

New England Electric

NORSTAR ENERGY, L.P.

Cowest Energy

Selkirk Cogeneration Partners

NORSTAR ENERGY, L..P.

Enron Gas Marketing Canada CNG Gas Services

NORSTAR ENERGY, L.P.

Renaissance Energy Ltd.

Medina Power Company

NORSTAR ENERGY, L..P,

Renaissance Energy Ltd.

New England Electric

NORSTAR ENERGY, L.P.

Renaissance Energy Lid.

Norstar Energy

NORSTAR ENERGY, L.P.

Western Gas Marketing Limited

MG Natural Gas Corporation

NORSTAR ENERGY, L.P.

Western Gas Marketing Limited

Medina Power Company

NORSTAR ENERGY, L.P.

Western Gas Marketing Limited National Fuel Gas Distribution

NORSTAR ENERGY, L.P.

Western Gas Marketing Limited New England Electric

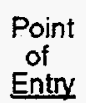

Eastport, Idaho

Waddington, NY

Waddington, NY

Waddington, NY

Niagara Falls, NY

Waddington, NY

Niagara Falls, NY

Waddington, NY

Niagara Falls, NY

Waddington, NY

- N/A

- N/A

$71 \quad 1.64$

$71 \quad 1.64$
Vol. Price

February

Vol. Price

$0 \quad N / A$

$0 \quad$ N/A

$512 \quad 1.36$

o N/A

- N/A

to 1.54

$10 \quad 1.54$

$0 \quad$ N/A

$5 \quad 1.60$

- N/A

$5 \quad 1.60$

$280 \quad 1.69$

o N/A

$280 \quad 1.69$

$9 \quad 2.29$

\begin{tabular}{l}
8.29 \\
\hline
\end{tabular}

$9 \quad 1.58$

27

2.05

156

- N/A

- N/A

$15 \quad 2.35$

15

2.35

$3 \quad 2.85$

$0 \quad$ N/A

- N/A

$3 \quad 2.85$

78

2.29

$7 \quad 1.48$

$\begin{array}{ll}78 & 2.29\end{array}$

162

2.25
- N/A

- N/A

$40 \quad 1.54$

$40 \quad 1.54$ 
SHORT-TERM SALES

YEAR: 1995 Quarter: One

Estimated Volumes (MMCF) \& Prices (\$ / MMBTU)

Importer

Seller

Purchaser / End User

NORSTAR ENERGY, L.P.

Western Gas Marketing Limited Norstar Energy

Niagara Falls, NY

Niagara Falls, NY

Western Gas Marketing Limited

Western Gas Marketing Inc.
Point
of

Entry
January
Vol. Price
February

Vol. Price
March
Vol. Price

Quarterly

Total

Avg. Price
NORTH AMERICAN RESOURCES COMPANY

Altana Exploration

North American Resources Company Babb, Montana

94

19

1.08

$53 \quad 1.10$

343

1.11

NORTH CANADIAN MARKETING CORPORATION

North Canadian Marketing Inc.

Cibola Corporation

Port of Morgan, MT

152

1.10

140

1.10

155

1.09

447

1.10

NORTH CANADIAN MARKETING CORPORATION

North Canadian Marketing Inc.

Coastal Gas Marketing Company

Port of Morgan, MT

464

420

1.10

$467 \quad 1.09$

1351

1.10

NORTH CANADIAN MARKETING CORPORATION

North Canadian Marketing Inc.

Michigan Consolidated Gas Company Noyes, Minnesota

2.43

202

2.38

$221 \quad 2.42$

638

NORTH CANADIAN MARKETING CORPORATION

North Canadian Marketing Inc.

Natural Gas Clearinghouse

Port of Morgan, MT

280

1.10

301

1.09

886

1.10

NORTH CANADIAN MARKETING CORPORATION

Various Suppliers

Amoco Trading

Eastport, Idaho

- N/A

- N/A

313

0.63

313

0.63

NORTH CANADIAN MARKETING CORPORATION

Various Suppliers

Associated Natural Gas

Eastport, Idaho

1083

0.87

0.90

2503

0.63

4707

NORTH CANADIAN MARKETING CORPORATION

Various Suppliers

Equitable Resources Marketing Compa Eastport, Idaho

$0 \quad$ N/A

195

0.90

36

0.63

231

0.86

NORTH CANADIAN MARKETING CORPORATION

Various Suppliers

Hercules

Eastport, Idaho

775

0.87

1117

0.90

$0 \quad N / A$

1891

0.89 
SHORT-TERM SALES

YEAR: 1995 Quarter: One

Estimated Volumes (MMCF) \& Prices ( $\$$ / MMBTU)

Importer

Seller

Purchaser/ End User
Point

Entry
January
Vol. Price
February
Vol. Price
March

Vol. Price
Quarterly

Vol. ${ }^{\text {Avg. }}$ Price

NORTH CANADIAN MARKETING CORPORATION

Vanous Suppliers

Kimball Energy Corporation

Eastport, Idaho

$\begin{array}{ll}68 & 0.87\end{array}$

- N/A

O N/A

68

0.87

NORTH CANADIAN MARKETING CORPORATION

Various Suppliers

North Canadian Marketing Corp.

Eastport, Idaho

$145 \quad 0.87$

154

0.90

$9 \quad 0.63$

308

0.88

NORTH CANADIAN MARKETING CORPORATION

Various Suppliers

Pacific Gas \& Electric Company

Eastport, Idaho

828

0.87

- N/A

O N/A

828

0.87

NORTH CANADIAN MARKETING CORPORATION

Various Suppliers

Sierra Pacific Power Company

Eastport, Idaho

400

0.87

363

0.90

407

0.63

1170

0.80

NORTH CANADIAN MARKETING CORPORATION

Various Suppliers

Tosco Energy

Eastport, Idaho

$\begin{array}{ll}62 & 0.87\end{array}$

O N/A

- N/A

62

0.87

NORTHERN MINNESOTA UTILITIES

ProGas Limited

Northem Minnesota Utilities

Noyes, Minnesota

$335 \quad 2.93$

161

2.44

- N/A

497

2.77

NORTHERN UTILITIES, INC.

Gaz Metropolitan, Inc.

Northem Utilities, Inc.

North Troy, VT

$50 \quad 1.86$

$89 \quad 1.86$

35

1.86

174

1.86

NORTHERN UTILITIES, INC.

Renaissance Energy L.td.

Northem Utilities, Inc.

Niagara Falls, NY

$29 \quad 1.50$

27

1.17

29

1.16

86

1.28

NORTHRIDGE GAS MARKETING, INC.

Northridge Gas Marketing

Alberta Northeast Gas Limited

Waddington, NY

- N/A

$10 \quad 1.60$

- N/A

10

1.60

NORTHRIDGE GAS MARKETING, INC.

Northridge Gas Marketing

Asarco Inc.

Babb, Montana

O N/A

O N/A

$2 \quad 1.37$

$2 \quad 1.37$

NORTHRIDGE GAS MARKETING, INC.

Northridge Gas Marketing

Coastal Gas Marketing Company

Noyes, Minnesota

- N/A

$2 \quad 1.07$

$0 \quad$ N/A

$2 \quad 1.07$ 
SHORT-TERM SALES

YEAR: 1995 Quarter: One

Estimated Volumes (MMCF) \& Prices (\$ / MMBTU)
Importer

Seller

Purchaser / End User

NORTHRIDGE GAS MARKETING, INC.

Northridge Gas Marketing

Coastal Gas Marketing Company

NORTHRIDGE GAS MARKETING, INC.

Northridge Gas Marketing

Columbia Falls Aluminum

NORTHRIDGE GAS MARKETING, INC. Northridge Gas Marketing

Continental Energy Marketing Ltd.

NORTHRIDGE GAS MARKETING, INC.

Northridge Gas Marketing

Holman inc.

Babb, Montana

January

of

Penitas, Texas

$158 \quad 1.36$

February
Vol. Price

March

Vol. Price

Quarterly

Total

Avg.

Vol. Price

$0 \quad$ N/A

$0 \quad$ N/A

$7 \quad 3.30$

$7 \quad 3.30$

Niagara Falls, NY

O N/A

O N/A

$32 \quad 1.73$

32

1.73

NORTHRIDGE GAS MARKETING, INC.

Northridge Gas Marketing

Interenergy Corporation

Babb, Montana

$3 \quad 0.75$

$2 \quad 0.79$

$0 \quad$ N/A

$5 \quad 0.77$

NORTHRIDGE GAS MARKETING, INC. Northridge Gas Marketing

John Brown Oil \& Gas

Babb, Montana

O N/A

O N/A

$13 \quad 0.89$

13

0.89

NORTHRIDGE GAS MARKETING, INC. Northridge Gas Marketing

John Brown Oil \& Gas

Noyes, Minnesota

O N/A

$1 \quad 1.11$

$0 \quad$ N/A

$1 \quad 1.11$

NORTHRIDGE GAS MARKETING, INC. Northridge Gas Marketing

Louisiana Pacific

Babb, Montana

$0 \quad$ N/A

o N/A

$6 \quad 1.31$

$6 \quad 1.31$

NORTHRIDGE GAS MARKETING, INC. Northridge Gas Marketing

Montana Resources

Babb, Montana

$12 \quad 1.46$

$10 \quad 1.65$

$4 \quad 1.45$

25

1.53

NORTHRIDGE GAS MARKETING, INC.

Northridge Gas Marketing

Northem Minnesota Utilities

Noyes, Minnesota

$155 \quad 1.37$

$0 \quad$ N/A

$0 \quad$ N/A

155

1.37

NORTHRIDGE GAS MARKETING, INC

Northridge Gas Marketing

Ocean State Power

Niagara Falls, NY $\begin{array}{llll}21 & 1.88 & 28 & 1.56\end{array}$

$0 \quad$ N/A

49

1.70 
SHORT-TERM SALES

YEAR: 1995 Quarter: One

Estimated Volumes (MMCF) \& Prices (\$ / MMBTU)
Importer

Seller

Purchaser / End User

NORTHRIDGE GAS MARKETING, INC.

Northridge Gas Marketing

Plum Creek Manufacturing

NORTHRIDGE GAS MARKETING, INC.

Northridge Gas Marketing

Progas

NORTHRIDGE GAS MARKETING, INC.

Northridge Gas Marketing

Rhone-Poulenc

Babb, Montana

49

January

of

Entry

Babb, Montana

Noyes, Minnesota

155

1.36

42

February

Vol. Price

March

Vol. Price

Quarterly

Total

Vol. Price

NORTHRIDGE GAS MARKETING, INC.

Northridge Gas Marketing

Stone Container Corporation

Babb, Montana

123

1.68

21.68

221

1.19

346

1.37

NORTHRIDGE GAS MARKETING, INC.

Northridge Gas Marketing

Union Gas Limited

Noyes, Minnesota

502

3.30

451

3.18

500

3.10

1453

3.19

NORTHRIDGE GAS MARKETING, INC

Northridge Gas Marketing

Western Gas Resources

Babb, Montana

$0 \quad$ N/A

- N/A

161

0.80

161

0.80

NOVAGAS CLEARINGHOUSE LTD.

Novagas Clearinghouse Ltd.

Acco Wortd-East

Massena, New York

11.95

11.95

21.95

NOVAGAS CLEARINGHOUSE LTD.

Novagas Clearinghouse Ltd.

Acco World-West

Massena, New York

$3 \quad 3.90$

23.90

$3 \quad 3.90$

8

3.90

NOVAGAS CLEARINGHOUSE LTD.

Novagas Clearinghouse Ltd.

Canton-Potsdam Hospital

Massena, New York

$2 \quad 1.95$

11.95

$2 \quad 1.95$

$5 \quad 1.95$

NOVAGAS CLEARINGHOUSE LTD.

Novagas Clearinghouse Ltd.

City of Ogdensburg, NY

Massena, New York

$1 \quad 1.95$

$0 \quad 1.95$

11.95

21.95

NOVAGAS CLEARINGHOUSE LTD.

Novagas Clearinghouse Ltd.

Hoosier Magnetics, Inc.

Massena, New York

$8 \quad 1.95$

$8 \quad 1.95$

$8 \quad 1.95$

24

1.95 
Importer

Seller

Purchaser/End User

NOVAGAS CLEARINGHOUSE LTD.

Novagas Clearinghouse Ltd.

Norfolk Paper Company

NOVAGAS CLEARINGHOUSE LTD.

Novagas Clearinghouse Ltd.

Stella Cheese Company

Massena, New York

NOVAGAS CLEARINGHOUSE LTD.

Novagas Clearinghouse Ltd.

United Helpers Management Company

NOVAGAS CLEARINGHOUSE LTD.

Novagas Clearinghouse Ltd.

Village of Potsdam Housing Auth.

NUMAC ENERGY (U.S.) INC.

Numac Energy

Associated Gas Services, Inc.

NUMAC ENERGY (U.S.) INC.

Numac Energy

Cibola Corporation

Port of Morgan, MT

16

1.18

$\begin{array}{ll}6 & 0.97\end{array}$

$9 \quad 0.95$

NUMAC ENERGY (U.S.) INC.

Numac Energy

Coastal Gas Marketing Company

NUMAC ENERGY (U.S.) INC

Numac Energy

Enron Gas Marketing

Port of Morgan, MT

$\begin{array}{ll}7 & 1.18\end{array}$

$15 \quad 0.97$

o N/A

22

1.04

NUMAC ENERGY (U.S.) INC.

Numac Energy

Tenaska Marketing Ventures

Port of Morgan, MT

$0 \quad$ N/A

- N/A

$8 \quad 0.95$

$8 \quad 0.95$

OCEAN STATE POWER

Northridge Gas Marketing

Ocean State Power

Niagara Falls, NY

.88

$14 \quad 1.56$

$0 \quad N / A$

$36 \quad 1.75$

OCEAN STATE POWER Renaissance Energy Ltd. Ocean State Power
Niagara Falls, NY
30

1.07

Quarterly
Total
Avg.
Vol $^{\text {Price }}$

$17 \quad 1.95$

1.95

1.95

$98 \quad 0.89$
$61 \quad 1.95$
$0 \quad N / A$

$0 \quad$ N/A

$14 \quad 1.56$ 


\section{SHORT-TERM SALES \\ YEAR: 1995 Quarter: One \\ Estimated Volumes (MMCF) \& Prices (\$ / MMBTU)}

Importer

Seller

Purchaser/End User

OCEAN STATE POWER II

Northridge Gas Marketing

Ocean State Power II

OCEAN STATE POWER \|

Renaissance Energy Ltd.

Ocean State Power II

Niagara Falls, NY

- N/A

$0 \quad$ N/A

$14 \quad 1.56$

14

1.56

PAN NATIONAL GAS SALES, INC.

Sonatrading

Associated Gas Services, inc.

Lake Charles, LA

$178 \quad 154$

$784 \quad 1.53$

19

1.36

1156

1.50

PAN-ALBERTA GAS (U.S.), INC

Northwest Pacific Energy Marketing

IGI Resources

Sumas, Washington

o N/A

O N/A

$13 \quad 1.19$

13

1.19

PAN-ALBERTA GAS (U.S.), INC

Northwest Pacific Energy Marketing James River Corporation

Sumas, Washington

1.18

189

1.28

203

1.19

600

1.21

PAN-ALBERTA GAS (U.S.), INC Northwest Pacific Energy Marketing Natural Gas Clearinghouse

Sumas, Washington

O N/A

- N/A

$41 \quad 1.19$

41

1.19

PAN-ALBERTA GAS (U.S.), INC.

Pan-Alberta Gas Ltd.

Associated Gas Services, Inc.

PAN-ALBERTA GAS (U.S.), INC

Pan-Alberta Gas Ltd.

Cenergy, Inc.

Eastport, Idaho

0.63

- N/A

- N/A

301

0.63

PAN-ALBERTA GAS (U.S.), INC

Pan-Alberta Gas Ltd.

Chevron U.S.A. Inc.

Eastport, Idaho

0.63

142

0.41

302

0.52

835

0.55

PAN-ALBERTA GAS (U.S.), INC.

Pan-Alberta Gas Ltd.

Continental Energy Marketing Ltd.

Eastport, Idaho

$57 \quad 0.63$

$0 \quad$ N/A

$88 \quad 0.52$

145

0.56

PAN-ALBERTA GAS (U.S.), INC.

Pan-Alberta Gas Ltd.

Enron Gas Marketing
Eastport, Idaho
$326 \quad 1.26$

0.41

- N/A

326

1.26 
SHORT-TERM SALES

YEAR: 1995 Quarter: One

Estimated Volumes (MMCF) \& Prices (\$ / MMBTU)
Importer

Seller

Purchaser / End User

PAN-ALBERTA GAS (U.S.), INC.

Pan-Alberta Gas Ltd.

Equitable Resources Marketing Compa

PAN-ALBERTA GAS (U.S.), INC.

Pan-Alberta Gas Ltd.

Inland Pacific

Eastport, Idaho

157

January
Vol. Price Vebruary
Vol. Price

Eastport, Idaho

34

$265 \quad 1.23$

$68 \quad 0.52$

675

1.17

PAN-ALBERTA GAS (U.S.), INC

Pan-Alberta Gas Ltd.

Morgan Stanley

PAN-ALBERTA GAS (U.S.), INC

Pan-Alberta Gas Ltd.

Natural Gas Clearinghouse

Eastport, Idaho

414

0.82

219

0.52

813

0.84

PAN-ALBERTA GAS (U.S.), INC.

Pan-Alberta Gas Ltd.

Natural Gas Clearinghouse

Sumas, Washington

- N/A

13

0.41

- N/A

13

0.41

PAN-ALBERTA GAS (U.S.), INC

Pan-Alberta Gas Ltd.

Pacific Gas \& Electric (UEG)

Eastport, Idaho

200

1.26

108

0.41

321

0.52

629

0.74

PAN-ALBERTA GAS (U.S.), INC.

Pan-Alberta Gas Ltd.

Pan-Alberta Gas (U.S.) Inc.

Eastport, Idaho

0.63

1665

0.41

- N/A

3084

0.51

PAN-ALBERTA GAS (U.S.), INC.

Pan-Alberta Gas Ltd.

Poco Petroleum

Eastport, Idaho

- N/A

o. N/A

$35 \quad 1.04$

35

1.04

PAN-ALBERTA GAS (U.S.), INC.

Pan-Alberta Gas Ltd.

Southwest Gas Comporation

Eastport, Idaho

- N/A

$2 \quad 0.41$

$94 \quad 0.52$

96

0.52

PAN-ALBERTA GAS (U.S.), INC.

Pan-Alberta Gas Ltd.

U.S. Gas Transportation Inc.

Eastport, Idaho

49

0.63

37

0.41

16

0.52

439

0.44

PAN-ALBERTA GAS (U.S.), INC.

Pan-Alberta Gas Ltd.

Utilicom United
Eastport, Idaho
102

0.41

- N/A

215

0.53 
SHORT-TERM SALES

YEAR: 1995 Quarter: One

Estimated Volumes (MMCF) \& Prices (\$ / MMBTU)
Importer

Seller

Purchaser / End User

PAN-ALBERTA GAS (U.S.), INC.

Pan-Alberta Gas Ltd.

Westcoast Gas Services (U.S.A.) Inc

Eastport, Idaho

- N/A

38

0.82

- N/A

38

0.82

PANCANADIAN PETROLEUM COMPANY PanCanadian Petroleum Ltd.

American Home Foods

Eastport, Idaho

$14 \quad 1.00$

11

0.87

14

0.84

40

0.91

PANCANADIAN PETROLEUM COMPANY PanCanadian Petroleum Ltd.

Anadarko Trading Comipany

Port of Morgan, MT

1298

1.13

1176

0.88

1307

0.87

378

0.96

PANCANADIAN PETROLEUM COMPANY

PanCanadian Petroleum Ltd.

Associated Gas Services, Inc

Eastport, Idaho

1.00

690

0.87

573

0.84

1725

0.89

PANCANADIAN PETROLEUM COMPANY PanCanadian Petroleum Ltd.

Califomia Protein Products

Eastport, Idaho

- N/A

90

0.87

- N/A

90

0.87

PANCANADIAN PETROLEUM COMPANY PanCanadian Petroleum Ltd.

Certainteed Corporation

Eastport, Idaho

$98 \quad 1.00$

O N/A

93

0.84

190

0.92

PANCANADIAN PETROLEUM COMPANY PanCanadian Petroleum Ltd.

Chevron U.S.A. Inc.

Eastport, Idaho

100

140

0.87

338

0.84

671

0.89

PANCANADIAN PETROLEUM COMPANY

PanCanadian Petroleum Ltd.

Coastal Gas Marketing Company

Eastport, ldaho

- N/A

O N/A

68

0.84

68

0.84

PANCANADIAN PETROLEUM COMPANY

PanCanadian Petroleum Ltd.

Com Products

Eastport, Idaho

$26 \quad 1.00$

18

0.87

34

0.84

78

0.90

PANCANADIAN PETROLEUM COMPANY PanCanadian Petroleum Ltd.

Continental Energy Marketing Ltd.

Eastport, Idaho

$0 \quad$ N/A

- N/A

218

0.84

218

0.84

PANCANADIAN PETROLEUM COMPANY PanCanadian Petroleum Ltd.

Cook Inlet

Eastport, Idaho
$324 \quad 1.00$

- N/A

21

0.84

538

0.94 
SHORT-TERM SALES

YEAR: 1995 Quarter: One

Estimated Volumes (MMCF) \& Prices (\$ / MMBTU)
Importer

Seller

Purchaser/End User

PANCANADIAN PETROLEUM COMPANY

PanCanadian Petroleum Ltd.

Dairyman's Cooperative Creamery

Eastport, Idaho

January

Vol. Price

February

of

Entry

PANCANADIAN PETROLEUM COMPANY PanCanadian Petroleum Ltd.

Equitable Resources Marketing Compa

PANCANADIAN PETROLEUM COMPANY PanCanadian Petroleum Ltd.

Evergreen Oil Inc.

Eastport, Idaho

$14 \quad 1.00$

13

0.87

$13 \quad 0.84$

40

0.91

PANCANADIAN PETROLEUM COMPANY PanCanadian Petroleum Ltd.

Koch Gas Services Company

PANCANADIAN PETROLEUM COMPANY

PanCanadian Petroleum Ltd.

L.P. McNear Brick Co., Inc.

Eastport, Idaho

- N/A

$8 \quad 0.87$

$9 \quad 0.84$

17

0.85

PANCANADIAN PETROLEUM COMPANY PanCanadian Petroleum Ltd.

Municipal Utility District

Port of Morgan, MT

168

1.13

143

0.88

159

0.87

469

0.97

PANCANADIAN PETROLEUM COMPANY PanCanadian Petroleum Ltd.

National Gas Resources

Eastport, Idaho

1.00

944

0.87

815

0.84

2610

0.90

PANCANADIAN PETROLEUM COMPANY PanCanadian Petroleum Ltd.

Nurserymen's Exchange

Eastport, Idaho

$18 \quad 1.00$

20

0.87

18

0.84

56

0.90

PANCANADIAN PETROLEUM COMPANY PanCanadian Petroleum Ltd.

Pacific Gas \& Electric Company

Eastport, idaho

$155 \quad 1.00$

O N/A

o N/A

$155 \quad 1.00$

PANCANADIAN PETROLEUM COMPANY PanCanadian Petroleum Ltd.

Poppy Foods

Eastport, Idaho

O N/A

O N/A

10.84

$1 \quad 0.84$
PANCANADIAN PETROLEUM COMPANY PanCanadian Petroleum Ltd.

Redwood Resources Inc.

Eastport, Idaho

(

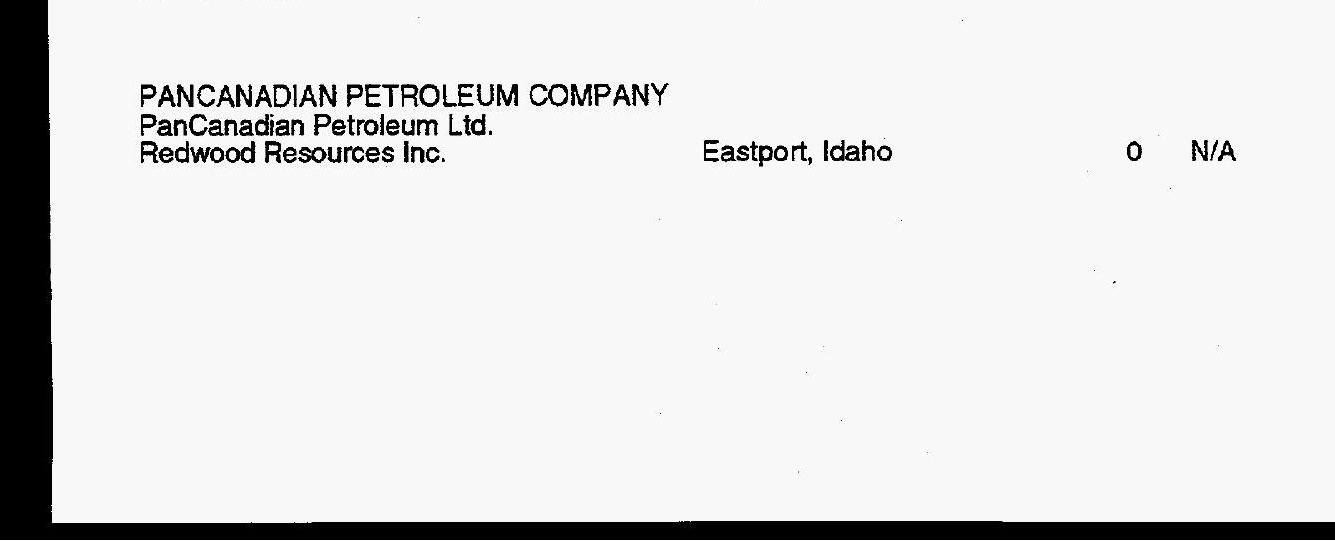


SHORT-TERM SALES

YEAR: 1995 Quarter: One

Estimated Volumes (MMCF) \& Prices ( $\$$ / MMBTU)

Importer

Seller

Purchaser / End User

PANCANADIAN PETROLEUM COMPANY PanCanadian Petroleurn Ltd.

Sierra Pacific Industries:

Eastport, Idaho

Port of Morgan, MT

PanCanadian Petroleum Ltd.

Unicorp Energy Inc.

PANCANADIAN PETROLEUM COMPANY

PanCanadian Petroleum Ltd.

Utilicorp United

Port of Morgan, MT

Eastport, Idaho

PanCanadian Petroleuin Ltd.

Vlasic Foods inc.

PANCANADIAN PETROLEUM COMPANY

PanCanadian Petroleum Ltd.

Washington Water Power Company

Eastport, Idaho

Eastport, Idaho

PARAMOUNT RESOURCES U.S., INC.

Paramount Resources Ltd.

Cook InletMock Resources

Eastport, Idaho

PARAMOUNT RESOURCES U.S., INC.

Paramount Resources Ltd.

Cook Inlet'National \& Riedwood Resou

Eastport, Idaho

PARAMOUNT RESOURCES U.S., INC.

Paramount Resources Ltd.

Cook Inlet/Redwood/Mock \& National

Eastport, Idaho

Waddington, NY

Brymore Energy, Ltd

Pawtucket Power Associates

PETRO-CANADA HYDROCARBONS, INC.

Petro-Canada Hydrocarbons inc.

AIG Trading Corporation

Sumas, Washington
$11 \quad 1.00$

$10 \quad 0.87$

14

0.84

34

0.90

o N/A

O N/A

896

0.52

896

0.52

- N/A

$3 \quad 1.74$

- N/A

3

1.74

Quarteriy
Total

Avg.

Vol. Price

$93 \quad 0.90$

$20 \quad 1.00$

$31 \quad 0.89$

$10 \quad 1.00$

$51 \quad 0.31$

$609 \quad 0.86$

$\begin{array}{llllllll}0 & \text { N/A } & 0 & \text { N/A } & 275 & 1.35 & 275 & 1.35\end{array}$ 
SHORT-TERM SALES

YEAR: 1995 Quarter: One

Estimated Volumes (MMCF) \& Prices ( $\$$ / MMBTU)
Importer

Seller

Purchaser / End User

PETRO-CANADA HYDROCARBONS, INC.

Petro-Canada Hydrocarbons Inc.

Coastal Gas Marketing Company

PETRO-CANADA HYDROCARBONS, INC.

Petro-Canada Hydrocarbons Inc.

Coastal Gas Marketing Company

PETRO-CANADA HYDROCARBONS, INC. Petro-Canada Hydrocarbons Inc.

Enron Gas Marketing

Eastport, Idaho

PETRO-CANADA HYDROCARBONS, INC. Petro-Canada Hydrocarbons Inc.

IGI Resources

Eastport, Idaho

$15 \quad 1.67$

- N/A

o N/A

$15 \quad 1.67$

PETRO-CANADA HYDROCARBONS, INC. Petro-Canada Hydrocarbons Inc.

IGI Resources

Sumas, Washington

308

1.43

279

1.18

401

1.35

988

1.33

PETRO-CANADA HYDROCARBONS, INC. Petro-Canada Hydrocarbons inc.

Kimball Energy Corporation

Sumas, Washington

260

$$
1.43
$$

$38 \quad 1.18$

$0 \quad$ N/A

298

1.40

PETRO-CANADA HYDROCARBONS, INC. Petro-Canada Hydrocarbons inc.

Mock Resources

Sumas, Washington

59

1.43

$0 \quad N / A$

$0 \quad N / A$

$59 \quad 1.43$

PETRO-CANADA HYDROCARBONS, INC. Petro-Canada Hydrocarbons inc.

Teco Gas Marketing

Sumas, Washington

$14 \quad 1.43$

- N/A

$11 \quad 1.35$

25

1.40

PETRO-CANADA HYDROCARBONS, INC. Petro-Canada Hydrocarbons inc.

Tenaska Gas Company

Sumas, Washington

$10 \quad 1.43$

$4 \quad 1.18$

21.35

$16 \quad 1.36$

PETRO-CANADA HYDROCARBONS, INC. Petro-Canada Hydrocarbons Inc.

Universal Resources

Sumas, Washington

$80 \quad 1.43$

$0 \quad \mathrm{~N} / \mathrm{A}$

- N/A

$80 \quad 1.43$

PETRO-CANADA HYDROCARBONS, INC.

Petro-Canada Hydrocartons inc.

Washington Natural Gas Company

Sumas, Washington
$319 \quad 1,43$

27

1.18

$15 \quad 1.35$

604

1.32 
SHORT-TERM SALES

YEAR: 1995 Quarter: One

Estimated Volumes (MMCF) \& Prices (\$ / MMBTU)

Importer

Seller

Purchaser / End User

PETRO-CANADA HYDROCARBONS, INC.

Petro-Canada Hydrocarbons inc.

Westcoast Gas Services (U.S.A.) Inc

Port of Morgan, MT

PG\&E ELECTRIC SUFPLY BUSINESS UNIT

Northstar Energy Corporation

PG\&E Electric Supply Business Unit

Eastport, Idaho

PG\&E GAS SUPPLY BUSINESS UNIT

AEC Oil \& Gas Company

PG\&E Gas Supply Business Unit

Eastport, Idaho

Eastport, Idaho

AIG Trading Corporation

PG\&E Gas Supply Business Unit

Point

January

Vol. Price

February

Vol. Price

March

Vol. Price

Quarterly

Total

Vol. ${ }^{\text {Avg. }}$ Price

451

404

1.29

$445 \quad 1.28$

1300

1.37

313

1.1

27

0.97

$314 \quad 1.93$

902

1.37

PG\&E GAS SUPPLY EUUSINESS UNIT Amoco Canada Petroleum Company Ltd. PG\&E Gas Supply Business Unit

Eastport, Idaho

120

1815

1.40

1276

1.44

4295

1.52

PG\&E GAS SUPPLY BUSINESS UNIT Bay Gas Marketing Inc.

PG\&E Gas Supply Business Unit

Eastport, Idaho

605

$\begin{array}{lll}0.97 & 282 & 1.59\end{array}$

o N/A

887

1.17

PG\&E GAS SUPPLY EUUSINESS UNIT Brymore Energy, Ltd.

PG\&E Gas Supply Business Unit

Eastport, Idaho

171

0.71

136

0.74

251

0.78

558

0.75

PG\&E GAS SUPPLY EUSINESS UNIT CanStates Gas Marketing

PG\&E Gas Supply Business Unit

Eastport, Idaho

- N/A

93

0.78

330

0.68

424

0.70

PG\&E GAS SUPPLY EUSINESS UNIT

Canadian Hunter Exploration Ltd.

PG\&E Gas Supply Business Unit

Eastport, Idaho

0.80

91

0.74

247

0.78

433

0.78

PG\&E GAS SUPPLY EUSINESS UNIT Canadian Occidental Fetroleum Ltd.

PG\&E Gas Supply Business Unit

Eastport, Idaho

184

0.71

331

0.68

19

0.77

535

0.69

PG\&E GAS SUPPLY BUUSINESS UNIT Cibola Canada Energy Marketing Co.

PG\&E Gas Supply Business Unit
Eastport, Idaho 


\section{SHORT-TERM SALES \\ YEAR: 1995 Quarter: One \\ Estimated Volumes (MMCF) \& Prices (\$/MMBTU)}

Importer

Seller

Purchaser/End User

PG\&E GAS SUPPLY BUSINESS UNIT Coastal Gas Marketing, Canada

PG\&E Gas Supply Business Unit

PG\&E GAS SUPPLY BUSINESS UNIT Continental Energy Marketing Ltd.

PG\&E Gas Supply Business Unit

PG\&E GAS SUPPLY BUSINESS UNIT Cook Inlet Energy Supply

PG\&E Gas Supply Business Unit

PG\&E GAS SUPPLY BUSINESS UNIT Crestar Energy

PG\&E Gas Supply Business Unit

Point
of

Entry

Eastport, Idaho

Eastport, Idaho

Eastport, Idaho

Eastport, Idaho

Eastport, Idaho

Direct Energy Marketing Limited
PG\&E Gas Supply Business Unit

PG\&E GAS SUPPLY BUSINESS UNIT Enron Capital \& Trade Resources Can PG\&E Gas Supply Business Unit

PG\&E GAS SUPPLY BUSINESS UNIT Enron Gas Marketing Canada

PG\&E Gas Supply Business Unit

PG\&E GAS SUPPLY BUSINESS UNIT Equitable

PG\&E Gas Supply Business Unit

Eastport, Idaho

PG\&E GAS SUPPLY BUSINESS UNIT Equitable Resources Marketing Canad PG\&E Gas Supply Business Unit

Eastport, Idaho

$94 \quad 0.74$

83

0.79

o N/A

176

0.76

PG\&E GAS SUPPLY BUSINESS UNIT Hesse Gas

PG\&E Gas Supply Business Unit

Eastport, Idaho

PG\&E GAS SUPPLY BUSINESS UNIT Institutional Capital Corporation

PG\&E Gas Supply Business Unit

January

Vol. Price

February

March

vol. Price

$224 \quad 0.77$

335

0.77

Total

Avg.

Vol. Price

$1454 \quad 1.91$

$1216 \quad 1.61$

$1252 \quad 1.64$

3922

Eastport, Idaho

2420

$0 \quad$ N/A

$0 \quad$ N/A

$39 \quad 0.74$

39

0.74

302. $\quad 1.44$

27

1.44

301

1.44

876

1.44

Eastport, Idaho
$317 \quad 2.33$

282

2.25

420

2.29

1019

2.29 


\section{SHORT-TERM SALES \\ YEAR: 1995 Quarter: One \\ Estimated Volumes (MMCF) \& Prices ( $/$ MMBTU)}

importer

Seller

Purchaser / End User

PG\&E GAS SUPPLY BUSINESS UNIT J. Aron \& Company

PG\&E Gas Supply Business Unit

PG\&E GAS SUPPLY BUSINESS UNIT

Mobil Oil Canada Ltd.

PG\&E Gas Supply Business Unit

PG\&E GAS SUPPLY BUSINESS UNIT

Morgan Stanley Capital Company

PG\&E Gas Supply Business Unit

PG\&E GAS SUPPLY BUSINESS UNIT

Natural Gas Clearinghcuse

PG\&E Gas Supply Business Unit

PG\&E GAS SUPPLY EUSINESS UNIT Northridge Gas Marketing

PG\&E Gas Supply Business Unit

Eastport, Idaho

Eastport, Idaho

$63 \quad 0.67$

- N/A

- N/A

63

0.67

PG\&E GAS SUPPLY EUSINESS UNIT

Novagas Clearinghouse Ltd.

PG\&E Gas Supply Business Unit

Eastport, Idaho

- N/A

39

0.77

o N/A

39

0.77

PG\&E GAS SUPPLY EUSINESS UNIT Pan-Alberta Gas Ltd.

PG\&E Gas Supply Business Unit

Eastport, Idaho

$914 \quad 1.81$

$1142 \quad 1.67$

$896 \quad 1.75$

2951

PG\&E GAS SUPPLY EUSINESS UNIT Petro Source Gas Ventures

PG\&E Gas Supply Business Unit

Eastport, Idaho

194

0.74

98

0.73

$53 \quad 0.79$

346

0.74

PG\&E GAS SUPPLY BUSINESS UNIT Petro-Canada Hydrocarbons inc.

PG\&E Gas Supply Business Unit

PG\&E GAS SUPPLY EUSINESS UNIT Poco Petroleum Ltd.

PG\&E Gas Supply Business Unit

Eastport, Idaho
$34 \quad 0.76$

- N/A

34

0.76 
SHORT-TERM SALES

YEAR: 1995 Quarter: One

Estimated Volumes (MMCF) \& Prices (\$ / MMBTU)
Importer

Seller

Purchaser / End User

PG\&E GAS SUPPLY BUSINESS UNIT

Premier Energy Services, Inc.

PG\&E Gas Supply Business Unit

PG\&E GAS SUPPLY BUSINESS UNIT Premier Enterprises, Inc.

PG\&E Gas Supply Business Unit

PG\&E GAS SUPPLY BUSINESS UNIT ProGas Limited

PG\&E Gas Supply Business Unit

Eastport, ldaho

PG\&E GAS SUPPLY BUSINESS UNIT Sceptre Resources Ltd.

PG\&E Gas Supply Business Unit

PG\&E GAS SUPPLY BUSINESS UNIT

Tristar Gas Marketing Company

PG\&E Gas Supply Business Unit

PG\&E GAS SUPPLY BUSINESS UNIT Wascana Energy Marketing Inc.

PG\&E Gas Supply Business Unit

PG\&E GAS SUPPLY BUSINESS UNIT Westcoast Gas Services Inc

PG\&E Gas Supply Business Unit

PG\&E GAS SUPPLY BUSINESS UNIT

Western Gas Resources

PG\&E Gas Supply Business Unit

Eastport, Idaho

$0 \quad$ N/A

- N/A

301

0.77

301

0.77

PG\&E GAS SUPPLY BUSINESS UNIT Western Gas Services

PG\&E Gas Supply Business Unit

Eastport, Idaho

2774

257

1.42

6238

1.36

$11590 \quad 1.48$

PHIBRO OIL \& GAS, INC.

Catex Energy Inc.

Iroquois Gas Transmission

PHIBRO OIL \& GAS, INC.

Coastal Gas Marketing, Canada

Louis Dreyfus Energy Corporation
Waddington, NY

$0 \quad$ N/A

140

1.65

O N/A

140

1.65

Niagara Falls, NY
523

3.81

199

1.98

530

3.64

1252

3.45 
SHORT-TERM SALES

YEAR: 1995 Quarter: One

Estimated Volumes (MMCF) \& Prices (\$ / MMBTU)
Importer

Seller

Purchaser / End User

PHIBRO OIL \& GAS, INC.

Coastal Gas Marketing, Canada Piedmont Natural Gas Company

PHIBRO OIL \& GAS, INC.

Enron Gas Marketing Canada

CoEnergy Trading Company

PHIBRO OIL \& GAS, INC.

Enron Gas Marketing Canada

Piedmont Natural Gas Company

PHIBRO OIL \& GAS, INC.

Equitable

Brooklyn Union Gas

PHIBRO OIL \& GAS, INC.

Equitable

CoEnergy Trading Company

PHIBRO OIL \& GAS, INC.

Equitable

Enron Gas Marketing

PHIBRO OIL \& GAS, INC.

Husky Gas Marketing inc.

New England Power Company

PHIBRO OIL \& GAS, INC. Louis Dreyfus Energy Corporation

Iroquois Energy Management

PHIBRO OIL \& GAS, INC.

Louis Dreyfus Energy Corporation Iroquois Gas Transmission

PHIBRO OIL \& GAS, INC.

Niagara Mowhawk Power Corporation

Louis Dreyfus Energy Corporation

PHIBRO OIL \& GAS, INC

ProGas Limited

New England Power Company

$$
\begin{aligned}
& \text { Point } \\
& \text { of } \\
& \text { Entry }
\end{aligned}
$$

Niagara Falls, NY

Niagara Falls, NY

$20 \quad 1.78$

- N/A

- N/A

20

1.78

Niagara Falls, NY

- N/A

224

1.53

$248 \quad 1.54$

472

1.54

Waddington, NY

$0 \quad$ N/A

$0 \quad$ N/A

310

2.12

310

2.12

Waddington, NY

310

2.12

o N/A

- N/A

310

2.12

Waddington, NY

- N/A

280

2.12

- N/A

280

2.12

Waddington, NY

o N/A

58

1.58

- N/A

58

1.58

Waddington, NY

- N/A

- N/A

310

1.70

310

1.70

Waddington, NY

$310 \quad 1.90$

280

1.73

- N/A

590

1.82

Niagara Falls, NY

- N/A

280

1.84

$0 \quad$ N/A

280

1.84

Niagara Falls, NY
- N/A

72

.62
- N/A
$72 \quad 1.62$ 


\section{SHORT-TERM SALES \\ YEAR: 1995 Quarter: One \\ Estimated Volumes (MMCF) \& Prices (\$ / MMBTU)}

Importer

Seller

Purchaser / End User

PHIBRO OIL \& GAS, INC.

Renaissance Energy Ltd.

CoEnergy Trading Company

PHIBRO OIL \& GAS, INC.

Renaissance Energy Ltd.

Equitable Resources

PHIBRO OIL \& GAS, INC.

Renaissance Energy Ltd.

Iroquois Energy Management

PHIBRO OIL \& GAS, INC.

Renaissance Energy Ltd.

Iroquois Gas Transmission

PHIBRO OIL \& GAS, INC.

Renaissance Energy Ltd.

New England Power Company

Waddington, NY

January

Vol. Price

February

of

Niagara Falls, NY

$161 \quad 1.81$

- N/A

O N/A

161

1.81

Waddington, NY

- N/A

$20 \quad 1.61$

$0 \quad N / A$

20

1.61

Waddington, NY

O N/A

- N/A

$155 \quad 1.54$

155

Waddington, NY

$6 \quad 1.81$

$51 \quad 1.57$

$88 \quad 1.55$

146

1.57

PHIBRO OIL \& GAS, INC.

Renaissance Energy Ltd.

Piedmont Natural Gas Company

Niagara Falls, NY

- N/A

154

1.61

- N/A

154

PHIBRO OIL \& GAS, INC.

Selkirk Cogeneration

Direct Energy Marketing

PHIBRO OIL \& GAS, INC.

Selkirk Cogeneration

Iroquois Gas Transmission

PHIBRO OIL \& GAS, INC.

Shell Canada Limited

CoEnergy Trading Company

PHIBRO OIL \& GAS, INC.

Wascana Energy Marketing Inc.

New England Power Company

PHIBRO OIL \& GAS, INC.

Western Gas Marketing Inc.

New England Power Company
Waddington, NY

$17 \quad 1.72$

o N/A

$0 \quad$ N/A

$17 \quad 1.72$

Niagara Falls, NY

$41 \quad 1.84$

- N/A

$0 \quad$ N/A

1.84

Niagara Falls, NY

$102 \quad 1.84$

$91 \quad 1.84$

O N/A

193

1.84

Waddington, NY
$0 \quad$ N/A

$0 \quad N / A$

301 


\section{SHORT-TERM SALES \\ YEAR: 1995 Quarter: One \\ Estimated Volumes (MMCF) \& Prices (\$ / MMBTU)}

Importer

Seller

Purchaser / End User

PHIBRO OIL \& GAS, INC

Western Gas Marketing Limited CoEnergy Trading Company

PHIBRO OIL \& GAS, INC

Western Gas Marketing Limited

Enron Gas Marketing

\section{Point \\ of \\ Entry}

Niagara Falls, NY

Waddington, NY

Waddington, NY

Niagara Falls, NY

Western Gas Marketing Limited

Piedmont Natural Gas Company

POCO PETROLEUM, INC.

Poco Petroleum Ltd.

AIG Trading Corporation

POCO PETROLEUM, INC.

Poco Petroleum Lid.

AmGas inc.

POCO PETROLEUM, INC.

Poco Petroleum Ltd.

Brooklyn Interstate Natural Gas

POCO PETROLEUM, INC.

Poco Petroleum Ltd.

Cibola Corporation

POCO PETROLEUM, INC.

Poco Petroleum Ltd.

Coastal Gas Marketing Company

POCO PETROLEUM, INC.

Poco Petroleum Ltd.

Coastal Gas Marketing Company

Noyes, Minnesota

Eastport, Idaho

Noyes, Minnesota
Waddington, NY

January

Vol. Price

February

Vol. Price

March

Vol. Price

Quarterty

Total

Avg.

Vol. Price

O N/A

$36 \quad 3.20$

- N/A

36

3.20

- N/A

117

1.10

- N/A

117

1.10

Port of Morgan, MT

$162 \quad 1.05$

148

0.83

$165 \quad 0.81$

474

0.90

Port of Morgan, MT

153

1.03

136

0.81

$151 \quad 0.79$

440

0.88

Port of Morgan, MT

$15 \quad 1.12$

- N/A

$0 \quad$ N/A

$15 \quad 1.12$

$30 \quad 0.98$

- N/A

- N/A

30

0.98 $\begin{array}{llll}0 & \text { N/A } & 14 \quad 1.08\end{array}$

$0 \quad N / A$ 


\section{SHORT-TERM SALES \\ YEAR: 1995 Quarter: One \\ Estimated Volumes (MMCF) \& Prices (\$ / MMBTU)}

Importer

Seller

Purchaser/End User

POCO PETROLEUM, INC.

Poco Petroleum Ltd.

Coastal Gas Marketing Company

POCO PETROLEUM, INC.

Poco Petroleum Ltd.

Cook Inlet

Eastport, Idaho

Eastport, Idaho

Port of Morgan, MT

$58 \quad 0.91$

$0 \quad$ N/A

- N/A

58

0.91

POCO PETROLEUM, INC.

Poco Petroleum Ltd.

IGI Resources

Eastport, Idaho

$140 \quad 1.00$

243

2.00

146

2.05

528

1.75

POCO PETROLEUM, INC.

Poco Petroleum Ltd.

IGI Resources

Sumas, Washington

$605 \quad 3.65$

546

3.47

568

3.47

1718

3.53

POCO PETROLEUM, INC.

Poco Petroleum Lid.

Koch Gas Services Company

Port of Morgan, MT

$0 \quad N / A$

$57 \quad 0.93$

- N/A

57

0.93

POCO PETROLEUM, INC.

Poco Petroleum Lid.

Koch Gas Services Company

POCO PETROLEUM, INC.

Poco Petroleum Ltd.

MichCon Trading

Noyes, Minnesota

$0 \quad$ N/A

O N/A

74

1.16

74

1.16

POCO PETROLEUM, INC.

Poco Petroleum Ltd.

Midcon Gas Services Corporation

Port of Morgan, MT

$0 \quad N / A$

O N/A

$69 \quad 0.86$

69

0.86

POCO PETROLEUM, INC.

Poco Petroleum Ltd.

Midland Cogeneration Venture
Noyes, Minnesota
$71 \quad 1.11$

$82 \quad 1.13$

153

1.12 


\section{SHORT-TERM SALES \\ YEAR: 1995 Quarter: One \\ Estimated Volumes (MMCF) \& Prices (\$ / MMBTU)}

Importer

Seller

Purchaser / End User

POCO PETROLEUM, INC.

Poco Petroleum Ltd.

Mobil Gas Marketing Inc.

POCO PETROLEUM, INC.

Poco Petroleum Ltd.

Natural Gas Clearinghouse

POCO PETROLEUM, INC.

Poco Petroleum Ltd.

Natural Gas Clearinghouse

POCO PETROLEUM, INC.

Poco Petroleum Ltd.

Neste Oy

POCO PETROLEUM, INC.

Poco Petroleum Ltd.

Neste Oy

$$
\begin{aligned}
& \text { Point } \\
& \text { of } \\
& \text { Entry }
\end{aligned}
$$

January

Vol. Price

February

Vol. Price

March

Vol. Price

Quarterly

Total

Avg.

Vol. Price

Eastport, Idaho

$8 \quad 0.99$

$3 \quad 0.84$

O N/A

11

0.95

Eastport, Idaho

$0 \quad$ N/A

105

0.85

- N/A

105

0.85

Sumas, Washington

$124 \quad 1.01$

$150 \quad 1.03$

$141 \quad 0.99$

414

1.01

Eastport, Idaho

o N/A

$16 \quad 0.98$

$99 \quad 1.10$

115

1.08

Port of Morgan, MT

$3 \quad 0.88$

$0 \quad$ N/A

$0 \quad N / A$

3

0.88

POCO PETROLEUM, INC.

Poco Petroleum Ltd.

Neste Oy

Sumas, Washington

70

1.10

121.00

$83 \quad 1.01$

164

1.05

POCO PETROLEUM, INC.

Poco Petroleum Ltd.

NorAm Energy Services

POCO PETROLEUM, INC

Poco Petroleum Ltd.

Northwest Natural Gas Company

Sumas, Washington

372

2.04

$414 \quad 1.83$

$613 \quad 1.60$

1399

1.79

POCO PETROLEUM, INC.

Poco Petroleum Ltd.

Pacific Gas \& Electric Company

Eastport, ldaho

105

0.89

168

0.85

420

0.86

692

0.86

POCO PETROLEUM, INC.

Poco Petroleum Ltd.

Sierra Pacific Power Company

Eastport, Idaho

- N/A

$5 \quad 0.95$

$0 \quad N / A$

$5 \quad 0.95$

POCO PETROLEUM, INC.

Poco Petroleum Ltd.

Sierra Pacific Power Company
Sumas, Washington
$0 \quad$ N/A

$0 \quad$ N/A

113

1.17 
Importer

Seller

Purchaser / End User

POCO PETROLEUM, INC.

Poco Petroleum Ltd.

Southwest Gas Corporation

POCO PETROLEUM, INC.

Poco Petroleum Ltd.

Teco Gas Marketing

POCO PETROLEUM, INC.

Poco Petroleum Ltd.

Valero industrial Gas

POCO PETROLEUM, INC.

Poco Petroleum Ltd.

Washington Natural Gas Company

Sumas, Washington

POCO PETROLEUM, INC.

Poco Petroleum Ltd.

Windward Energy \& Marketing Inc.

Eastport, Idaho

POWER CITY PARTNERS, L.P.

Husky Oil

Power City Partners, L.P.

Waddington, NY

PROGAS U.S.A., INC.

ProGas Limited

AIG Trading Corporation

PROGAS U.S.A., INC.

ProGas Limited

CMS Gas Marketing

Noyes, Minnesota

PROGAS U.S.A., INC.

ProGas Limited

Chautauqua Energy Marketing Inc.

Niagara Falls, NY

PROGAS U.S.A., INC.

ProGas Limited

Cibola Corporation

Noyes, Minnesota

PROGAS U.S.A., INC.

ProGas Limited

Cibola Corporation
Port of Morgan, MT
55

2.52

28

282
- N/A

o N/A

25

0.90

25

0.90

$2 \quad 1.20$

o N/A

O N/A

21.20

$25 \quad 1.67$

$14 \quad 1.65$

$5 \quad 1.61$

$44 \quad 1.66$

- N/A

o N/A

153

1.10

153

1.10

$61 \quad 1.86$

$55 \quad 1.72$

61

1.76

177

1.78

- N/A

- N/A

327

1.10

327

1.10

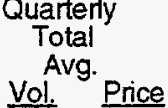

8.89

3.40

484

2.5


SHORT-TERM SALES

YEAR: 1995 Quarter: One

Estimated Volumes (MMCF) \& Prices (\$ / MMBTU)
Importer

Seller

Purchaser / End User

PROGAS U.S.A., INC.

ProGas Limited

City of Perham, MN

PROGAS U.S.A., INC.

ProGas Limited

CoEnergy Trading Company

PROGAS U.S.A., INC.

ProGas Limited

Coastal Gas Marketing Company

PROGAS U.S.A., INC.

ProGas Limited

Direct Energy Marketing

PROGAS U.S.A., INC.

ProGas Limited

Great Plains Natural Gas Company

PROGAS U.S.A., INC.

ProGas Limited

KCS Energy Management

PROGAS U.S.A., INC.

ProGas Limited

KCS Energy Management

PROGAS U.S.A., INC.

ProGas Limited

Midland Cogeneration Venture

PROGAS US.A., INC.

ProGas Limited

Natural Gas Clearinghouse

PROGAS U.S.A., INC.

ProGas Limited

NorAm Energy Services

PROGAS U.S.A., INC.

ProGas Limited

Phibro Oil \& Gas Inc.

$$
\text { Point }
$$

Entry

Noyes, Minnesota

Noyes, Minnesota

Noyes, Minnesota

Noyes, Minnesota

Noyes, Minnesota

Niagara Falls, NY

Noyes, Minnesota

Noyes, Minnesota

Port of Morgan, MT

Port of Morgan, MT

Niagara Falls, NY
January

Vol. Price

February

Vol Price

March

Vol. Price

Quarterly

Total

Vol. Price

$56 \quad 2.20$

$59 \quad 2.20$

175

2.39

400

2.76

118

1.10

310

1.10

828

1.90

348

1.38

155

1.10

- N/A

503

1.29

$26 \quad 1.38$

- N/A

- N/A

26

1.38

271

4.14

287

2.20

252

2.20

810

2.85

$90 \quad 1.86$

81

1.72

90

1.76

261

1.78

$4 \quad 1.38$

$2 \quad 1.10$

- NIA

$7 \quad 1.28$

140

1.10

15

1.10

391

1.17
$81 \quad 0.94$

- N/A

- N/A

81

0.94
$439 \quad 1.86$
458

1.76 


\section{SHORT-TERM SALES \\ YEAR: 1995 Quarter: One \\ Estimated Volumes (MMCF) \& Prices (\$ / MMBTU)}

Importer

Seller

Purchaser / End User

\section{Point \\ of}

Entry

Noyes, Minnesota

Port of Morgan, MT

Niagara Falls, NY

Port of Morgan, MT

Noyes, Minnesota

ProGas Limited

Westcoast Gas Services (U.S.A.) Inc

RENAISSANCE ENERGY (U.S.), INC. Renaissance Energy Ltd.

Renaissance Energy (U.S.) Inc.

RENAISSANCE ENERGY (U.S.), INC.

Renaissance Energy Ltd.

Renaissance Energy (U.S.) Inc.

RENAISSANCE ENERGY (U.S.), INC.

Renaissance Energy Ltd.

Renaissance Energy (U.S.) Inc.

RENAISSANCE ENERGY (U.S.), INC.

Renaissance Energy Ltd.

Renaissance Energy (U.S.) Inc.

Port of Morgan, MT

$1347 \quad 1.48$

O N/A

1239

1.25

2586

1.37

RENAISSANCE ENERGY (U.S.), INC.

Renaissance Energy Ltd.

Renaissance Energy (U.S.) Inc.

Waddington, NY

$947 \quad 1.96$

860

1.74

1319

1.70

3126

1.79

SALMON RESOURCES LTD.

Shell Canada Limited

Cibola Corporation
Port of Morgan, MT
Quarterly

Total

Vol. Price

Voi. Price

$609 \quad 2.13$

$248 \quad 0.84$

$475 \quad 1.94$

$457 \quad 1.73$

$869 \quad 1.63$

$310 \quad 3.71$ 
SHORT-TERM SALES
YEAR: 1995 Quarter: One

Estimated Volumes (MMCF) \& Prices (\$/MMBTU)
Importer

Seller

Purchaser / End User

Point

Entry

Sumas, Washington

Eastport, Idaho

Eastport, Idaho

Eastport, Idaho

Waddington, NY

Waddington, NY

Northridge Gas Marketing

SELKIRK COGEN PAFTTNERS, L.P. Orange \& Rockland Energy

Selkirk Cogeneration Partners

SELKIRK COGEN PAFRTNERS, L.P. Renaissance Energy Lid.

Selkirk Cogeneration Partners

SIERRA PACIFIC POWER COMPANY

Petro-Canada Hydrocarbons Inc.

Sierra Pacific Power Company

ST. CLAIR PIPELINES LTD.

St. Clair Pipelines Ltd.

Midcon Marketing Corporation

Waddington, NY

Waddington, NY

Detroit, Michigan
Niagara Falls, NY

Selkirk Cogeneration Partners
January
Vol. Price Vobruary
Vol. Price

March

Vol. Price

Quarterly

Total

Vol. Price

$147 \quad 1.30$

$\begin{array}{llllll}51 & 1.57 & 46 & 1.35 & 50 & 0.99\end{array}$

$10 \quad 1.60$

$10 \quad 1.56$

O N/A

$20 \quad 1.58$

955

1.16

847

1.02

962

1.11

2764

1.10

366

1.16

138

1.10

367

0.94

$871 \quad 1.06$

- N/A

193

0.81

193

0.81

- N/A

$5 \quad 2.00$

- N/A

5

2.00

0 N/A

o N/A

$1 \quad 1.68$

1

1.68

Sumas, Washington

$460 \quad 1.75$

415

1.71

459

1.66

1334

1.71

335

1.85

73

1.85

409

1.85
- N/A 


\section{SHORT-TERM SALES \\ YEAR: 1995 Quarter: One \\ Estimated Volumes (MMCF) \& Prices (\$ / MMBTU)}

Importer

Purchaser / End User

ST. CLAIR PIPELINES LTD.

St. Clair Pipelines Lidd.

Midcon Marketing Corporation

ST. CLAIR PIPELINES LTD.

St. Clair Pipelines Ltd.

Minnegasco

ST. CLAIR PIPELINES LTD.

St. Clair Pipelines Lid.

Minnegasco

St. Clair, MI

Noyes, Minnesota

$0 \quad$ N/A

- N/A

22

1.11

22

1.11

SUNCOR INC.

Suncor lnc.

Associated Gas Services, Inc.

Eastport, Idaho

$0 \quad$ N/A

363

0.64

310

0.43

673

0.54

SUNCOR INC.

Suncor Inc.

Associated Natural Gas

Port of Morgan, MT

$310 \quad 1.16$

$280 \quad 0.91$

310

0.89

900

0.99

SUNCOR INC.

Suncor inc.

Bridgegas

Port of Morgan, MT

$2 \quad 1.16$

$0 \quad N / A$

- N/A

2

1.16

SUNCOR INC.

Suncor Inc.

Cibola Corporation

Port of Morgan, MT

$290 \quad 1.16$

$280 \quad 0.91$

310

0.89

880

0.99

SUNCOR INC.

Suncor inc.

City of Palo Alto, CA

Eastport, Idaho

$389 \quad 1.98$

$357 \quad 1.28$

340

0.86

108

1.40

SUNCOR INC.

Suncor Inc.

Coastal Gas Marketing Company

Eastport, Idaho

136

0.99

$197 \quad 0.64$

22

0.86

562

0.81

SUNCOR INC.

Suncor Inc.

IGI Resources
Eastport, Idaho
$280 \quad 0.64$

310

0.43

899

0.69 


\section{SHORT-TERM SALES \\ YEAR: 1995 Quarter: One \\ Estimated Volumes (MMCF) \& Prices (\$ / MMBTU)}

Importer

Seller

Purchaser / End User

SUNCOR INC.

Suncor lnc.

Inland Steel Company

SUNCOR INC.

Suncor Inc.

Metropolitan Utilities District

\section{Point}

of

Entry

Port of Morgan, MT

Port of Morgan, MT

$158 \quad 1.16$

143

0.91

158

0.89

459

0.99

SUNCOR INC.

Suncor inc.

Midcon Marketing Corporation

Port of Morgan, MT

$0 \quad$ N/A

- N/A

$151 \quad 0.89$

151

0.89

SUNCOR INC.

Suncor Inc.

Midwest Gas Company

SUNCOR INC.

Suncor Inc.

Minnegasco

SUNCOR INC.

Suncor lnc.

National Gas Resources

Eastport, Idaho

296

0.99

152

0.64

310

0.43

758

0.69

SUNCOR INC.

Suncor inc

Redwood Resources Inc.

SUNCOR INC.

Suncor Inc.

Shell Oil Company

SUNCOR INC.

Suncor Inc.

Wisconsin Power \& Light

Port of Morgan, MT

$343 \quad 1.16$

310

0.91

342

0.89

994

0.99

SUNRISE ENERGY COMPANY Associated Gas Services, Inc. Sunrise Energy

Eastport, Idaho

128

1.26

11

1.02

18

1.68

430

1.38

SUNRISE ENERGY COMPANY Continental Energy Marketing Ltd. Sunrise Energy
Eastport, Idaho
$67 \quad 1.22$

52

0.84

- N/A

119

1.05 
SHORT-TERM SALES

YEAR: 1995 Quarter: One

Estimated Volumes (MMCF) \& Prices (\$ / MMBTU)
Importer

Seller

Purchaser / End Use

SUNRISE ENERGY COMPANY

Maple View Gas Marketing

Sunnise Energy

TECO GAS MARKETING COMPANY

Talisman Energy, Inc.

Teco Gas Marketing

TENNECO GAS MARKETING COMPANY AEC Oil \& Gas Company

Inland Pacific

Eastport, Idaho

Eastport, Idaho

$10 \quad 0.97$

$0 \quad$ N/A

$0 \quad N / A$

10

0.97

AEC OIl \& Gas Company
Pacific Gas \& Electric Company

TENNECO GAS MARKETING COMPANY

AEC Oil \& Gas Company

Portland General Electric Company

Eastport, Idaho

0.97

O N/A

$0 \quad N / A$

$66 \quad 0.97$

TENNECO GAS MARKETING COMPANY AEC Oil \& Gas Company

Union Pacific Fuels

Eastport, Idaho

$41 \quad 0.97$

O N/A

O N/A

$41 \quad 0.97$

TENNECO GAS MARKETING COMPANY AlG Trading Corporation

Consolidated Edison

Niagara Falls, NY

$16 \quad 1.80$

O N/A

- N/A

$16 \quad 1.80$

TENNECO GAS MARKETING COMPANY Cibola Canada Energy Marketing Co.

Inland Pacific

Eastport, Idaho

$0 \quad$ N/A

64

0.86

$0 \quad$ N/A

$64 \quad 0.86$

TENNECO GAS MARKETING COMPANY Cibola Canada Energy Marketing Co.

Koch Gas Services Company

Eastport, Idaho

O N/A

$4 \quad 0.86$

o N/A

4

0.86

TENNECO GAS MARKETING COMPANY Cibola Canada Energy Marketing Co.

Natural Gas Clearinghouse

Eastport, Idaho

$0 \quad$ N/A

$5 \quad 0.86$

- N/A

$5 \quad 0.86$

TENNECO GAS MARKETING COMPANY

Renaissance Energy Ltd.

Pepperell Power Associates, L.P.

Niagara Falls, NY
$284 \quad 1.85$

- N/A

- N/A

284

1.85 


\section{SHORT-TERM SALES \\ YEAR: 1995 Quarter: One \\ Estimated Volumes (MMCF) \& Prices (\$ / MMBTU)}

Importer

Seller

Purchaser / End User

TENNECO GAS MARKIETING COMPANY

Western Gas Services

Tennessee Gas Pipeline Company

Eastport, Idaho

Eastport, Idaho

112

1.32

$83 \quad 1.37$

$89 \quad 1.39$

285

1.36

THE MONTANA POWER COMPANY

Enron Gas Marketing Canada

The Montana Power Company

Babb, Montana

$213 \quad 1.59$

186

1.59

$214 \quad 1.59$

613

1.59

TRANSCO ENERGY MARKETING COMPANY

CanStates Gas Marketing

Allied Producers Gas Service, Inc.

Niagara Falls, NY

O N/A

O N/A

$19 \quad 1.40$

$19 \quad 1.40$

TRANSCO ENERGY MARKETING COMPANY

CanStates Gas Marketing

$\mathrm{BNG}$, inc.

Niagara Falls, NY

TRANSCO ENERGY MARKETING COMPANY

CanStates Gas Marketing

Brooklyn Union Gas

Niagara Falls, NY

$1 \quad 2.01$

$1 \quad 3.08$

- N/A

$2 \quad 2.51$

TRANSCO ENERGY MARKETING COMPANY

CanStates Gas Marketing

CanStates Gas Marketing

Niagara Falls, NY

116

2.10

141

2.05

$163 \quad 1.91$

420

2.01

TRANSCO ENERGY MIARKETING COMPANY

CanStates Gas Marketing

Catex Vitol

Niagara Falls, NY

TRANSCO ENERGY MARKETING COMPANY

CanStates Gas Marketing

Clinton - Newbery

Niagara Falls, NY

TRANSCO ENERGY MARKETING COMPANY

CanStates Gas Marketing

Coastal Gas Marketing Company Niagara Falls, NY
1.66

TRANSCO ENERGY MARKETING COMPANY

CanStates Gas Marketing

Delmarva Power \& Light Company

Niagara Falls, NY
$213 \quad 1.59$

280

1.62

704

$211 \quad 1.78$

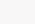

- N/A

$9 \quad 1.74$

101

1.64

110 
SHORT-TERM SALES

YEAR: 1995 Quarter: One

Estimated Volumes (MMCF) \& Prices (\$ / MMBTU)
Importer

Seller

Purchaser / End User
Point

Entry

TRANSCO ENERGY MARKETING COMPANY

CanStates Gas Marketing

Hazelton Fuel Management Co.

Niagara Falls, NY

$231 \quad 3.08$

$63 \quad 3.08$

$2 \quad 3.08$

296

3.08

TRANSCO ENERGY MARKETING COMPANY

CanStates Gas Marketing

Hopewell Cogeneration Facility

Niagara Falls, NY

O N/A

$5 \quad 2.45$

O N/A

5

TRANSCO ENERGY MARKETING COMPANY

CanStates Gas Marketing

Koch Gas Services Company

Niagara Falls, NY

- N/A

$8 \quad 3.03$

- N/A

8

3.03

TRANSCO ENERGY MARKETING COMPANY

CanStates Gas Marketing

New England Power Company

Niagara Falls, NY

- N/A

$6 \quad 1.61$

$15 \quad 1.58$

21

1.59

TRANSCO ENERGY MARKETING COMPANY

CanStates Gas Marketing

New York Power Authority

Niagara Falls, NY

$10 \quad 2.25$

- N/A

- N/A

10

2.25

TRANSCO ENERGY MARKETING COMPANY

CanStates Gas Marketing

Philadelphia Gas Works

Niagara Falls, NY

O N/A

$8 \quad 1.66$

O N/A

$8 \quad 1.66$

TRANSCO ENERGY MARKETING COMPANY

CanStates Gas Marketing

Public Service Electric \& Gas

Niagara Falls, NY

TRANSCO ENERGY MARKETING COMPANY

CanStates Gas Marketing

SL Knit

Niagara Falls, NY

TRANSCO ENERGY MARKETING COMPANY

CanStates Gas Marketing

Southem Connecticut Gas Company Niagara Falls, NY

TRANSCO ENERGY MARKETING COMPANY

CanStates Gas Marketing

Sun Refining

Niagara Falls, NY

TRANSCO ENERGY MARKETING COMPANY

CanStates Gas Marketing

Tenneco Gas Marketing

Niagara Falls, NY

O N/A

$92 \quad 1.15$

- N/A

92

1.15

o N/A

o. N/A

$14 \quad 1.83$

14

1.83

$4 \quad 2.11$

- N/A

O N/A

o N/A

o N/A

$86 \quad 1.69$

86

1.69

- N/A

- N/A

$60 \quad 1.81$

60

1.81 
SHORT-TERM SALES

YEAR: 1995 Quarter: One

Estimated Volumes (MMCF) \& Prices (\$ / MMBTU)
Importer

Seller

Purchaser / End User
Point

Entry
January
Vol. Price
February

Vol. Price
Quarterly
Total

Avg.

Vol. Price

TRANSCO ENERGY MARKETING COMPANY

CanStates Gas Marketing

U.S. Energy

Niagara Falls, NY

$280 \quad 1.60$

$232 \quad 1.43$

March

Vol. Plice

TRANSCO ENERGY MARKETING COMPANY

CanStates Gas Marketing

UGI Utilities

Niagara Falls, NY

$66 \quad 2.04$

$122 \quad 1.84$

$134 \quad 1.86$

321

1.89

TRANSCO ENERGY MARKETING COMPANY

CanStates Gas Marketing

Volunteer Energy Corporation

Niagara Falls, NY

$0 \quad$ N/A

$0 \quad$ N/A

$67 \quad 1.49$

67

1.49

TRISTAR GAS MARKETING COMPANY

Tristar Gas Marketing Company

Southem Califomia Gas Company

Eastport, Idaho

$51 \quad 0.93$

- N/A

- N/A

51

0.93

TRISTAR GAS MARKETING COMPANY

Tristar Gas Marketing Company

Tristar Gas Marketing

Eastport, Idaho

- N/A

O N/A

50.90

5

0.90

UNION PACIFIC FUELS, INC.

Union Pacific Resources Company

Union Pacific Fuels

Sumas, Washington

253

1.40

$244 \quad 1.03$

$453 \quad 1.00$

950

1.11

UTILICORP UNITED

Coastal Gas Marketing, Canada

Broad Street Oil \& Gas

Eastport, Idaho

- N/A

- N/A

$119 \quad 0.82$

119

0.82

UTILICORP UNITED

ProGas Limited

Utilicorp United

Noyes, Minnesota

- N/A

$140 \quad 4.60$

$334 \quad 4.09$

475

4.24

VECTOR ENERGY (U.S.A.) INC.

Vector Energy Inc.

Pacific Gas \& Electric Company

Eastport, Idaho

0.67

468

0.66

$518 \quad 0.68$

$1504 \quad 0.67$

VERMONT GAS SYSTEMS, INC.

Gaz Metropolitan, Inc.

Vermont Gas Systems, Inc.

Highgate Spr., VT

$4 \quad 14.5$

$18 \quad 5.52$

O N/A

22

7.19

VERMONT GAS SYSTEMS, INC.

Renaissance Energy Ltd.

Vermont Gas Systems, Inc.

Highgate Spr., VT

- N/A

O N/A

$23 \quad 1.80$

23

1.80 


\section{SHORT-TERM SALES \\ YEAR: 1995 Quarter: One \\ Estimated Volumes (MMCF) \& Prices (\$/MMBTU)}

Importer

Seller

Purchaser / End User

VERMONT GAS SYSTEMS, INC.

Western Gas Marketing Limited

Vermont Gas Systems, Inc.

Highgate Spr., VT

WASCANA ENERGY MARKETING (U.S.) INC.

Wascana Energy Marketing Inc.

Associated Gas Services, Inc.

Eastport, Idaho

- N/A

$0 \quad$ N/A

$8 \quad 1.01$

$8 \quad 1.01$

WASCANA ENERGY MARKETING (U.S.) INC.

Wascana Energy Marketing Inc.

Associated Gas Services, inc.

Port of Morgan, MT

- N/A

$0 \quad$ N/A

$152 \quad 1.23$

152

1.23

WASCANA ENERGY MARKETING (U.S.) INC.

Wascana Energy Marketing Inc.

Cibola Corporation

Port of Morgan, MT

148

1.34

57

1.19

38

2.72

243

1.52

WASCANA ENERGY MARKETING (U.S.) INC.

Wascana Energy Marketing Inc.

Coastal Gas Marketing Company

Eastport, Idaho

189

0.92

- N/A

- N/A

189

0.92

WASCANA ENERGY MARKETING (U.S.) INC.

Wascana Energy Marketing Inc.

Direct Gas Marketing

Grand Island, NY

$94 \quad 1.79$

$82 \quad 1.64$

$91 \quad 1.65$

268

1.70

WASCANA ENERGY MARKETING (U.S.) INC.

Wascana Energy Marketing Inc.

Enron Gas Marketing

Eastport, Idaho

WASCANA ENERGY MARKETING (U.S.) INC.

Wascana Energy Marketing inc.

IGI Resources

Eastport, Idaho

WASCANA ENERGY MARKETING (U.S.) INC.

Wascana Energy Marketing Inc.

Koch Gas Services Company

Eastport, Idaho

O N/A

- N/A

$9 \quad 0.94$

$9 \quad 0.94$

WASCANA ENERGY MARKETING (U.S.) INC.

Wascana Energy Marketing Inc.

Koch Gas Services Company

Port of Morgan, MT

$119 \quad 1.50$

O N/A

$0 \quad$ N/A

$119 \quad 1.50$

WASCANA ENERGY MARKETING (U.S.) INC

Wascana Energy Marketing Inc.

North Coast Energy, Inc.

Niagara Falls, NY
O N/A

$67 \quad 1.65$

1.66

$140 \quad 1.66$ 
SHORT-TERM SALES

YEAR: 1995 Quarter: One

Estimated Volumes (MMCF) \& Prices (\$ / MMBTU)
Importer

Seller

Purchaser / End User
Point

of

Entry
January

Vol. Price
February

Vol. Price
Quarterly

Total. Avg. Vol. Price

WASCANA ENERGY MARKETING (U.S.) INC.

Wascana Energy Marketing Inc.

Northwest Natural Gas Company

WASCANA ENERGY MARKETING (U.S.) INC.

Wascana Energy Marketing Inc.

Pacific Gas \& Electric Company

WASCANA ENERGY MARKETING (U.S.) INC.

Wascana Energy Marketing inc.

Phibro Oil \& Gas Inc.

Niagara Falls, NY

WASCANA ENERGY MARKETING (U.S.) INC.

Wascana Energy Marketing Inc.

Phibro Oil \& Gas Inc.

Port of Morgan, MT

154

1.52

$0 \quad$ N/A

$0 \quad$ N/A

154

1.52

WASCANA ENERGY MARKETING (U.S.) INC.

Wascana Energy Marketing Inc.

Prairieland Energy Marketing

Port of Morgan, MT

$0 \quad$ N/A

- N/A

$3 \quad 1.18$

3

1.18

WASCANA ENERGY MARKETING (U.S.) INC.

Wascana Energy Marketing Inc.

Prairieland Energy Marketing

Portal, ND

WASCANA ENERGY MARKETING (U.S.) INC.

Wascana Energy Marketing Inc.

Southwest Gas Corporation

Eastport, Idaho

o N/A

- N/A

$0 \quad 1.17$

0

1.17

WASCANA ENERGY MARKETING (U.S.) INC.

Wascana Energy Marketing Inc.

Tenaska Marketing Ventures

Port of Morgan, MT

WASCANA ENERGY MARKETING (U.S.) INC.

Wascana Energy Marketing Inc.

Tenneco Gas Marketing

Port of Morgan, MT

283

4.70

153

3.03

156

1.53

591

3.43

WASCANA ENERGY MARKETING (U.S.) INC.

Wascana Energy Marketing inc.
U.S. Gas Transportation Inc.

Port of Morgan, MT

$10 \quad 2.44$

10

1.36

52

1.35

WASCANA ENERGY MARKETING (U.S.) INC.

Wascana Energy Marketing Inc.

Western Gas Resources

Portal, ND
$58 \quad 1.11$

$\begin{array}{ll}71 & 0.92\end{array}$

59

0.92

188

0.98 


\section{SHORT-TERM SALES \\ YEAR: 1995 Quarter: One \\ Estimated Volumes (MMCF) \& Prices (\$ / MMBTU)}

\begin{tabular}{lc} 
Importer & Point \\
Seller & of \\
Purchaser / End User & Entry \\
\hline
\end{tabular}

WASHINGTON ENERGY GAS MARKETING COMPANY

IGI Resources

Washington Energy Gas Marketing Com Eastport, Idaho

$355 \quad 1.18$

January

Vol. Price

February

Vol. Price

March

Vol. Price

208

0.84

269

1.66

831

1.25

WASHINGTON NATURAL GAS COMPANY

Talisman LT

Washington Natural Gas Company

Sumas, Washington

$233 \quad 1.46$

$210 \quad 1.09$

23

1.06

673

1.21

WESTCOAST GAS SERVICES (U.S.A.), INC.

Westcoast Gas Services Inc.

Westcoast Gas Services (U.S.A.) Inc

St. Clair, M!

$47 \quad 1.61$

O N/A

$0 \quad N / A$

47

1.61

WESTCOAST GAS SERVICES INC

Westcoast Gas Services inc.

Arco Products Company

Sumas, Washington

$0 \quad$ N/A

0. N/A

$71 \quad 1.00$

71

1.00

WESTCOAST GAS SERVICES INC

Westcoast Gas Services Inc.

Canwest Gas Supply

Sumas, Washington

- N/A

$5 \quad 1.19$

O N/A

$5 \quad 1.19$

WESTCOAST GAS SERVICES INC.

Westcoast Gas Services Inc.

Inland Pacific

Sumas, Washington

$1 \quad 1.25$

$0 \quad$ N/A

$79 \cdot 0.94$

80

0.94

WESTCOAST GAS SERVICES INC.

Westcoast Gas Services Inc.

Kimball Energy Corporation

Sumas, Washington

$217 \quad 130$

196

1.11

227

1.11

640

1.17

WESTCOAST GAS SERVICES INC.

Westcoast Gas Services inc.

Natural Gas Clearinghouse

Noyes, Minnesota

$55 \quad 124$

$55 \quad 1.33$

79

1.18

188

1.24

WESTCOAST GAS SERVICES INC

Westcoast Gas Services Inc.

Norcen Energy Resources

Noyes, Minnesota

- N/A

- N/A

$1 \quad 1.20$

$1 \quad 1.20$

WESTCOAST GAS SERVICES INC.

Westcoast Gas Services Inc.

Poco Petroleum

Sumas, Washington

$0 \quad$ N/A

O N/A

$4 \quad 1.00$

$4 \quad 1.00$

WESTCOAST GAS SERVICES INC.

Westcoast Gas Services inc.

Potsdam College

Massena, New York
$16 \quad 160$

$15 \quad 1.60$

$16 \quad 1.60$

47

1.60 
importer

Seller

Purchaser / End User

WESTCOAST GAS SERVICES INC.

Westcoast Gas Services inc.

Potters Industries

WESTCOAST GAS SERVICES INC. Westcoast Gas Services Inc.

St. Lawrence University

WESTCOAST GAS SERVICES INC.

Westcoast Gas Services Inc.

Washington Natural Gas Company

WESTCOAST GAS SERVICES INC.

Westcoast Gas Services Inc.

Westcoast Gas Services (U.S.A.) inc

Eastport, Idaho

Point
of

Entry

Massena, New York

Massena, New York

Sumas, Washington

Noyes, Minnesota

Westcoast Gas Services Inc.

Westcoast Gas Services (U.S.A.) Inc

WESTCOAST GAS SERVICES INC.

Westcoast Gas Services inc.

Westcoast Gas Services (U.S.A.) Inc

WESTCOAST GAS SERVICES INC.

Westcoast Gas Services inc.

Westcoast Gas Services (U.S.A.) Inc

WESTERN GAS MARKETING INC. Western Gas Marketing Limited AIG Trading Corporation

WESTERN GAS MARKETING INC. Westem Gas Marketing Limited Associated Gas Services, Inc.

WESTERN GAS MARKETING INC. Western Gas Marketing Limited CNG Energy Services

Waddington, NY

Waddington, NY
SHORT-TERM SALES

YEAR: 1995 Quarter: One

Estimated Volumes (MMCF) \& Prices ( $\$$ / MMBTU) 
SHORT-TERM SALES

YEAR: 1995 Quarter: One

Estimated Volumes (MMCF) \& Prices ( $\$$ / MMBTU)
Importer

Seller

Purchaser / End User

WESTERN GAS MARKETING INC. Western Gas Marketing Limited Cenergy, inc.

WESTERN GAS MARKETING INC. Western Gas Marketing Limited Cenergy, Inc.

WESTERN GAS MARKETING INC. Western Gas Marketing Limited Cibola Corporation

Noyes, Minnesota

WESTERN GAS MARKETING INC. Western Gas Marketing Limited Cibola Corporation

WESTERN GAS MARKETING INC

Western Gas Marketing Limited

CoEnergy Trading Company

WESTERN GAS MARKETING INC.

Western Gas Marketing Limited

Coastal Gas Marketing Company

WESTERN GAS MARKETING INC.

Western Gas Marketing Limited

Coastal Gas Marketing Company

WESTERN GAS MARKETING INC.

Western Gas Marketing Limited

Coastal Gas Marketing Company

WESTERN GAS MARKETING INC.

Western Gas Marketing Limited

Coastal Gas Marketing Company

WESTERN GAS MARKETING INC.

Western Gas Marketing Limited

Consumers Power Company

Noyes, Minnesota

- N/A

- N/A

330

1.29

330

1.29

WESTERN GAS MARKETING INC.

Westem Gas Marketing Limited

Eastem Energy Marketing

Niagara Falls, NY

January

Vol. Price

February

Vol. Price

March

Price

Quarterly

Total

Vol. ${ }^{\text {Avg. }}$ Price

Noyes, Minnesota

- N/A

$0 \quad$ N/A

$156 \quad 1.29$

$156 \quad 1.29$

Port of Morgan, MT

$479 \quad 1.07$

425

0.93

158

0.90

1062

0.99

Noyes, Minnesota

$0 \quad$ N/A

- N/A

59

1.29

$59 \quad 1.29$

Eastport, Idaho
Grand Island, NY
Noyes, Minnesota

o N/A

- N/A

57

0.80

571

0.80

Eastport, Idaho
Grand Island, NY
Noyes, Minnesota

o N/A

- N/A

149

1.56

149

1.56

Eastport, Idaho
Grand Island, NY
Noyes, Minnesota

$789 \quad 1.53$

783

785

2357

1.38

Port of Morgan, MT

$167 \quad 1.07$

$0 \quad$ N/A

- N/A

$167 \quad 1.07$

NY

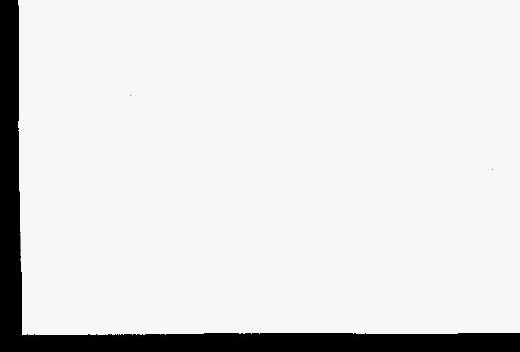




\section{SHORT-TERM SALES \\ YEAR: 1995 Quarter: One}

Estimated Volumes (MMCF) \& Prices (\$ / MMBTU)

Importer

Seller

Purchaser / End User

WESTERN GAS MARKETING INC.

Western Gas Marketing Limited

Eastem Energy Marketing

WESTERN GAS MARKETING INC.

Western Gas Marketing Limited

Eastem Energy Marketing

WESTERN GAS MARKETING INC.

Western Gas Marketing Limited

Enron Gas Marketing

Port of Morgan, MT

January

Vol. Price

February

Vol. Price

March

of

Noyes, Minnesota

- N/A

- N/A

$76 \quad 1.29$

76

1.29

Port of Morgan, MT

- N/A

$14 \quad 0.93$

0 N/A

14

0.93

WESTERN GAS MARKETING INC.

Western Gas Marketing Limited

Equitable Resources

Eastport, Idaho

- N/A

- N/A

$216 \quad 0.80$

216

0.80

WESTERN GAS MARKETING INC.

Western Gas Marketing Limited

Equitable Resources

Port of Morgan, MT

280

1.07

285

0.93

630

0.90

1195

0.95

WESTERN GAS MARKETING INC.

Western Gas Marketing Limited

Equitable Resources

Waddington, NY

- N/A

$49 \quad 1.72$

- N/A

$49 \quad 1.72$

WESTERN GAS MARKETING INC. Western Gas Marketing Limited

Hadson Gas Marketing

Port of Morgan, MT

187

1.07

16

0.93

$0 \quad N / A$

353

1.00

WESTERN GAS MARKETING INC Westem Gas Marketing Limited

IGI Resources

Eastport, Idaho

307

1.10

274

0.83

310

0.80

891

0.91

WESTERN GAS MARKETING INC.

Western Gas Marketing Limited

Koch Gas Services Coimpany

Eastport, Idaho

- N/A

136

0.83

151

0.80

287

0.81

WESTERN GAS MARKETING INC.

Western Gas Marketing Limited

Michigan Consolidated Gas Company

Noyes, Minnesota

338

1.53

278

1.15

$153 \quad 1.29$

769

1.35

WESTERN GAS MARIKETING INC.

Western Gas Marketing Limited

Midcon Gas Services Corporation
Noyes, Minnesota
70

1.53

o N/A

- N/A

70

1.53 
SHORT-TERM SALES

YEAR: 1995 Quarter: One

Estimated Volumes (MMCF) \& Prices ( $\$$ / MMBTU)
Importer

Seller

Purchaser / End User

WESTERN GAS MARKETING INC. Western Gas Marketing Limited

Migas Exchange

WESTERN GAS MARKETING INC.

Western Gas Marketing Limited

Minnegasco

Noyes, Minnesota

January

of

Port of Morgan, MT

$201 \quad 1.07$

309

0.93

191

0.90

701

0.96

WESTERN GAS MARKETING INC

Western Gas Marketing Limited

Natural Gas Clearinghouse

Noyes, Minnesota

$184 \quad 1.53$

606

1.31

308

1.29

1099

1.34

WESTERN GAS MARKETING INC. Western Gas Marketing Limited Neste Oy

Port of Morgan, MT

$161 . \quad 1.07$

211

0.93

158

0.90

531

0.96

WESTERN GAS MARKETING INC. Western Gas Marketing Limited NorAm Energy Services

Port of Morgan, MT

$0 \quad$ N/A

O N/A

42

0.90

42

0.90

WESTERN GAS MARKETING INC.

Westem Gas Marketing Limited

North Canadian Marketing Corp.

WESTERN GAS MARKETING INC.

Western Gas Marketing Limited

Northwest Natural Gas Company

WESTERN GAS MARKETING INC. Westem Gas Marketing Limited

O \& R Energy

Niagara Falls, NY

O N/A

$0 \quad$ N/A

$13 \quad 1.62$

13

1.62

WESTERN GAS MARKETING INC.

Western Gas Marketing Limited

Pacific Gas \& Electric Company

WESTERN GAS MARKETING INC.

Western Gas Marketing Limited

Paragon Gas Marketing

Waddington, NY

$41 \quad 1.97$

$37 \quad 1.72$

$41 \quad 1.63$

120

1.78

WESTERN GAS MARKETING INC.

Western Gas Marketing Limited

Peoples Natural Gas Company
St. Clair, MI
- N/A

40

2.28

40

2.28 


\section{SHORT-TERM SALES \\ YEAR: 1995 Quarter: One \\ Estimated Volumes (MMCF) \& Prices (\$ / MMBTU)}

Importer

Seller

Purchaser / End User

WESTERN GAS MARKETING INC.

Western Gas Marketing Limited

Poco Petroleum

WESTERN GAS MARKETING INC.

Westem Gas Marketing Limited

Sierra Pacific Power Company

WESTERN GAS MARKETING INC.

Westerm Gas Marketing Limited

Tenaska Marketing Veritures

WESTERN GAS MARKETING INC.

Western Gas Marketing Limited

Transco Energy Marketing Company

WESTERN GAS MARKETING INC.

Western Gas Marketing Limited

Washington Water Power Company

WESTERN GAS MARKETING INC.

Western Gas Marketing Limited

Westcoast Gas Services (U.S.A.) Inc

Eastport, Idaho

- N/A

218

0.83

$0 \quad$ N/A

218

0.83

WESTERN GAS MARKETTING INC.

Western Gas Marketing Limited

Westcoast Gas Services (U.S.A.) Inc

Noyes, Minnesota

O N/A

$0 \quad N / A$

$160 \quad 1.29$

160

1.29

WESTERN GAS MARKETING INC.

Western Gas Marketing Limited

Wisconsin Gas

Noyes, Minnesota

$17 \quad 1.53$

$29 \quad 1.31$

$32 \quad 1.29$

78

1.35

WESTERN GAS RESOURCES, INC.

Westcoast Gas Services inc.

Cenex, Inc.

Babb, Montana

$62 \quad 1.55$

- N/A

- N/A

$62 \quad 1.55$

WESTERN GAS RESCURCES, INC.

Westcoast Gas Services Inc.

Western Gas Resources

Babb, Montana

$54 \quad 2.82$

$56 \quad 1.65$

258

2.45

367

2.38

WESTERN GAS RESOURCES, INC Westcoast Gas Services Inc.

Western Sugar
Babb, Montana
- N/A
$39 \quad 1.55$ 


\section{SHORT-TERM EXPORTERS}

Estimated Volumes (MMCF)

1 SOURCE ENERGY SERVICES COMPANY

AIG TRADING CORPORATION

AMOCO ENERGY TRADING CORPORATION

ANADARKO TRADING COMPANY

ANTHEM ENERGY COMPANY, L.P.

BC GAS UTILITY LTD.

BRYMORE ENERGY INC.

CENTRA GAS ONTARIO INC.

CHANNEL GAS MARKETING COMPANY

CHEVRON U.S.A. INC.

CNG PRODUCING COMPANY

COASTAL GAS MARKETING COMPANY

COENERGY TRADING COMPANY

CONOCO INC.

CONSUMERS' GAS COMPANY LIMITED

CONTINENTAL ENERGY MARKETING LTD.

ECNG INC.

EL PASO GAS MARKETING COMPANY

EMC GAS TRANSMISSION COMPANY

ENRON GAS MARKETING, INC.

HOWARD ENERGY COMPANY, INC.

INLAND GAS \& OIL CORPORATION

INLAND PACIFIC ENERGY SERVICES LTD.

MERIDIAN OIL TRANSPORTATION INC.

MOBIL. NATURAL GAS INC.

NATURAL GAS CLEARINGHOUSE INC.

NORTH AMERICAN RESOURCES COMPANY

ORYX GAS MARKETING, L.P.

TENNECO GAS MARKETING COMPANY

TEXACO GAS MARKETING INC.

UMC PETROLEUM CORPORATION

UNION GAS LIMITED

UNION PACIFIC FUELS, INC.

VALERO INDUSTRIAL GAS, L.P.

WESTCOAST GAS SERVICES (U.S.A.), INC.

WESTERN GAS MARKETING INC.
1994

Jan.-Mar.

0

5893

977

0

0

0

0

3349

0

0

0

76

310

236

7014

166

0
390

326

880

0

501

0

894

0

121

100

239

0

131

3444

0

610

342

0

25998
1994
Apr-Jun

Apr-Jun.

111
72
12

1891

0

$$
0
$$

304

688

2510

1275

1037

0

0

0

0

363

0

0

0

216

0

0

0

204

166

0

1471

3197

0

697

342

253

16571

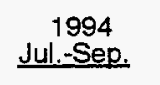

1994
Oct.-Dec.

1995
Jan.-Mar.

TOTALS

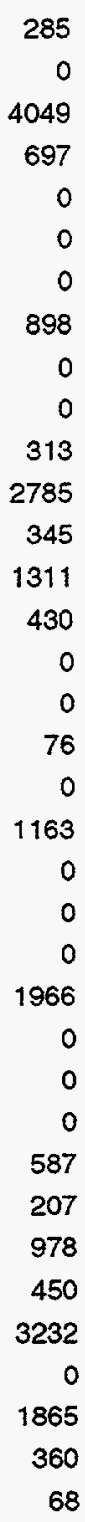

22066

\begin{tabular}{|c|c|}
\hline 294 & 0 \\
\hline 1517 & 572 \\
\hline 9734 & 9262 \\
\hline 600 & 58 \\
\hline 0 & 157 \\
\hline 425 & 439 \\
\hline 101 & 0 \\
\hline 1342 & 1183 \\
\hline 200 & 888 \\
\hline 1508 & 4138 \\
\hline 0 & 64 \\
\hline 1945 & 146 \\
\hline 1750 & 1010 \\
\hline 1328 & 1321 \\
\hline 0 & 0 \\
\hline 0 & 0 \\
\hline 104 & 166 \\
\hline 328 & 277 \\
\hline 0 & 0 \\
\hline 1879 & 361 \\
\hline 0 & 204 \\
\hline 75 & 0 \\
\hline 81 & 0 \\
\hline 352 & 0 \\
\hline 625 & 236 \\
\hline 302 & 0 \\
\hline 0 & 0 \\
\hline 501 & 0 \\
\hline 458 & 0 \\
\hline 302 & 0 \\
\hline 450 & 379 \\
\hline 3232 & 3162 \\
\hline 50 & 0 \\
\hline 1078 & 880 \\
\hline 89 & 79 \\
\hline 468 & 50 \\
\hline
\end{tabular}

31116 
PURCHASERS OF SHORT-TERM EXPORTS Estimated Volumes Exported (MMCF)

AGRI Foods International

Altana Exploration

BC Buildings Corporation

$B C$ Gas Inc.

BC Gas Utility Ltd.

Centra Gas Ontario inc.

Coast Mountain Hardwoods

Consumers' Gas Company Limited

Continental Energy Marketing Ltd.

Cowest Energy

Crown Packaging

Direct Energy Marketing

ECNG inc.

Enron Gas Marketing

Enron Gas Services Canada

Ewos Canada Ltd.

Gaz Metropolitain

Howard Energy Company, Inc.

Inland Pacific

MacMillan Bloedel

Naliey's Canada

Northridge Gas Marketing inc.

Ocean Construction Supplies

Panabode Intemational

PEMEX

Petro Canada Hydrocarbons

St. Clair Pipelines Ltd.

Suncor inc.

Thomas J. Lipton Inc.

Titan Steel and Wire Company

Union Gas Limited

Westcoast Gas Services Inc.

Western Gas Marketing Limited

Western Gas Services

Workers' Compensation Board of BC

Totals
1994

Jan.-Mar

0

100

0

501

0

3729

0

10564

166

0

0

0

0

131

0

0

0

0

0

0

0

0

0

0

4372

52

233

789

0

$\begin{array}{rr}0 & 0 \\ 5363 & 2986\end{array}$

$0 \quad 0$

$0 \quad 170$

$0 \quad 595$

0

25998

1165

0
397

0

62

181

0

0

0

0

0

936

0

0

4411

0

0

705

16571
1994
Apr.-Jun.

1994
Jul.-Sep.

1994

Oct.-Dec.

1995

Jan.-Mar.

Jul.-Sep.

0

0

0

0

0

898

0

550

0

225

0

$0 \quad 0$

2519

360

0

68

0

$\begin{array}{rr}26 & 0 \\ 0 & 0 \\ 7 & 0 \\ 75 & 0 \\ 425 & 439\end{array}$

$1342 \quad 1183$

110

$0 \quad 0$

$0 \quad 330$

$528 \quad 630$

80

$0 \quad 0$

$0 \quad 166$

$0 \quad 0$

$0 \quad 0$

$7 \quad 0$

$1323 \quad 50$

0204

690

120

$7 \quad 0$

$450 \quad 379$

40

$6 \quad 0$

$21483 \quad 17788$

$0 \quad 0$

$3 \quad 0$

$\begin{array}{rr}6 & 0 \\ 4508 & 3733\end{array}$

$101 \quad 79$

$468 \quad 50$

$\begin{array}{ll}0 & 0 \\ 7 & 0\end{array}$

$\underline{31116} \quad \underline{25032}$
$0 \quad 0$

$240 \quad 0$ 
SHORT-TERM SALES

YEAR: 1995 Quarter: One

Estimated Volumes (MMCF) \& Prices (\$ / MMBTU)

Exporter

Seller

Purchaser / End User

AIG TRADING CORPORATION

AIG Trading Corporation

Union Gas Limited

St. Clair, Michigan

AMOCO ENERGY TRADING CORPORATION

Amoco Energy Trading Corporation

PEMEX

Douglas, Arizona

El Paso, Texas

Amoco Energy Trading Corporation

PEMEX

AMOCO ENERGY TRADING CORPORATION

Amoco Energy Trading Corporation

PEMEX

Hidalgo, Texas

AMOCO ENERGY TRADING CORPORATION

Amoco Energy Trading Corporation

PEMEX

Penitas, Texas

January
Vol. Price

February
Vol. Price

$103 \quad 1.62$

$0 \quad$ N/A

$469 \quad 1.48$

572

Quarterly

Total

Vol. Price

222

1.14

$274 \quad 1.06$

496

1.10

602

1.64

818

1.33

1035

1.34

2455

1.41
ANADARKO TRADING COMPANY Anadarko Trading Company

PEMEX

Hidalgo, Texas

El Paso, Texas

$0 \quad$ N/A

0

N/A

157

1.38

157

1.38

BC GAS UTILITY LTD.

Amoco Energy Trading Corporation

BC Gas Utility Ltd.

Sumas, Washington

$60 \quad 1.46$

50

1.10

0

N/A

110

1.30

BC GAS UTILITY LTD.

Conoco Inc.

BC Gas Utility Ltd.

Sumas, Washington

90

25

1.11

$0 \quad$ N/A

$115 \quad 1.35$

BC GAS UTIUTY LTD

Phillips Gas Marketing Company

BC Gas Utility Ltd.

Sumas, Washington

25

1.10

0

N/A

115

1.38

BC GAS UTILITY LTD.

Union Oil of Califomia

$\mathrm{BC}$ Gas Utility Ltd.

Sumas, Washington

$74 \quad 1.42$

25

1.11

0

N/A

$99 \quad 1.34$

CENTRA GAS ONTARIO INC.

Anadarko Trading Company

Centra Gas Ontario Inc.

Detroit, Michigan 
SHORT-TERM SALES

YEAR: 1995 Quarter: One

Estimated Volumes (MMCF) \& Prices ( $\$$ / MMBTU)

Exporter

Seller

Purchaser / End User

CENTRA GAS ONTARIO INC

Associated Gas Services, Inc.

Centra Gas Ontario Inc.

CENTRA GAS ONTARIO INC

Tenaska Gas Company

Centra Gas Ontario Inc.

CHANNEL GAS MARKETING COMPANY Channel Gas Marketing Company

PEMEX

El Paso, Texas

CHANNEL GAS MARKETING COMPANY Channel Gas Marketing Company

PEMEX

Hidalgo, Texas

January

Vol. Price

February

Vol. Price

March

Exit

Detroit, Michigan

Detroit, Michigan

$0 \quad$ N/A

280

1.71

0

N/A

280

1.71

CHEVRON U.S.A. INC

Chevron U.S.A. Inc.

PEMEX

Penitas, Texas

1304

1.55

109

1.30

1735

1.36

4138

1.40

CNG PRODUCING COMIPANY

CNG Energy Services Corporation

PEMEX

Hidalgo, Texas

O N/A

64

1.37

0

N/A

64

1.37

COASTAL GAS MARKETING COMPANY Coastal Gas Marketing Company

PEMEX

El Paso, Texas

$10 \quad 1.65$

0

N/A

0

N/A

10

1.65

COASTAL GAS MARKETING COMPANY Coastal Gas Marketing Company PEMEX

Hidaigo, Texas

O N/A

36

4.17

60

1.55

96

2.53

COASTAL GAS MARKETING COMPANY Coastal Gas Marketing Company PEMEX

Penitas, Texas

1.46

10

1.26

20

2.68

40

2.02

COENERGY TRADING COMPANY

CoEnergy Trading Company

Continental Energy Marketing Ltd.

COENERGY TRADING COMPANY

CoEnergy Trading Company

Cowest Energy

St. Clair, Michigan

$217 \quad 1.80$

196

1.65

217

1.60

630

1.68

COENERGY TRADING COMPANY

CoEnergy Trading Company

Gaz Metropolitain

St. Clair, Michigan $\begin{array}{llll}0 & \text { N/A } & 50 \quad 1.65\end{array}$

$0 \quad$ N/A

$50 \quad 1.65$ 
SHORT-TERM SALES

YEAR: 1995 Quarter: One

Estimated Volumes (MMCF) \& Prices (\$ / MMBTU)

Exporter

Seller

Purchaser / End User

CONOCO INC.

Conoco inc.

PEMEX

ECNG INC.

Associated Gas Services, Inc.

ECNG Inc.

EL PASO GAS MARKETING COMPANY El Paso Gas Marketing Company

PEMEX

Point
of

Exit

Penitas, Texas

Sumas, Washington

El Paso, Texas

Douglas, Arizona

Penitas, Texas

Detroit, Michigan

Penitas, Texas

Harve, Montana

St. Clair, Michigan

Detroit, Michigan

Union Gas Limited

UNION GAS LIMITED

Westcoast Gas Services Inc.

Union Gas Limited

VALERO INDUSTRIAL GAS, L.P.

Valero Industrial Gas, L.P.

PEMEX

ENRON GAS MARKETING, INC

Enron Gas Marketing, Inc.

促

Mas inc.

UMC PETROLEUM CORPORATION

Petroleum Corporation

UNION GAS LIMITED

CoEnergy Trading Company

Union Gas Limited

St. Clair, Michigan
Westcoast Gas Services Inc.

January
Vol. Price

March
Vol.

Vol. Price

Quarterty

Avg.

Vol. Price

$456 \quad 1.45$

$408 \quad 1.26$

457

1.28

1321

1.33

108

1.50

58

1.02

0

N/A

166

1.33

0 N/A 192

2.79

85

1.39

277

2.36

$240 \quad 1.44$

0

N/A

0

N/A

$240 \quad 1.44$

2.32

560

2.35

620

2.33

1800

2.33

- N/A

0

N/A

468

2.26

469

2.26
$83 \quad 1.76$

67

1.57

72

1.58

222

1.64 


\section{SHORT-TERM SALES \\ YEAR: 1995 Quarter: One \\ Estimated Volumes (MMCF) \& Prices (\$ / MMBTU)}

Exporter
Seller
Purchaser / End User

VALERO INDUSTRIAL GAS, L.P.

Valero Industrial Gas, L.P.

PEMEX

VALERO INDUSTRIAL GAS, L.P.

Valero Industrial Gas, L.P.

PEMEX

Penitas, Texas

WESTCOAST GAS SERVICES (U.S.A.), INC.

Westcoast Gas Services (U.S.A.), Inc.

Westcoast Gas Services inc.

St. Clair, Michigan

27

January

Vol. Price

Hidalgo, Texas

0

N/A

February
Vol. Price

March

Vol. Price

Quarterly

Total

Avg.

Vol.

Price

O N/A

561

1.37

0

N/A

561

1.37

WESTERN GAS MARKETING INC.

Western Gas Marketing Inc.

Western Gas Marketing Limited

St. Clair, Michigan
N/A

0

N/A

50

2.15

50

2.15 